\title{
Psychophysical investigations of perceptual learning and attention
}

Citation for published version (APA):

Lange, G. F. (2016). Psychophysical investigations of perceptual learning and attention: new insights into old principles. [Doctoral Thesis, Maastricht University]. Maastricht University.

https://doi.org/10.26481/dis.20160226gl

Document status and date:

Published: 01/01/2016

DOI:

10.26481/dis.20160226gl

Document Version:

Publisher's PDF, also known as Version of record

\section{Please check the document version of this publication:}

- A submitted manuscript is the version of the article upon submission and before peer-review. There can be important differences between the submitted version and the official published version of record.

People interested in the research are advised to contact the author for the final version of the publication, or visit the DOI to the publisher's website.

- The final author version and the galley proof are versions of the publication after peer review.

- The final published version features the final layout of the paper including the volume, issue and page numbers.

Link to publication

\footnotetext{
General rights rights.

- You may freely distribute the URL identifying the publication in the public portal. please follow below link for the End User Agreement:

www.umlib.nl/taverne-license

Take down policy

If you believe that this document breaches copyright please contact us at:

repository@maastrichtuniversity.nl

providing details and we will investigate your claim.
}

Copyright and moral rights for the publications made accessible in the public portal are retained by the authors and/or other copyright owners and it is a condition of accessing publications that users recognise and abide by the legal requirements associated with these

- Users may download and print one copy of any publication from the public portal for the purpose of private study or research.

- You may not further distribute the material or use it for any profit-making activity or commercial gain

If the publication is distributed under the terms of Article $25 \mathrm{fa}$ of the Dutch Copyright Act, indicated by the "Taverne" license above, 
Psychophysical investigations of perceptual learning and attention

New insights into old principles

\author{
Gesa Lange
}


(C) Gesa Lange, Maastricht 2016

All rights reserved. No part of this publication may be reproduced, stored in a retrieval system or transmitted in any form or by any means, electronic, mechanical, photocopying, recording or otherwise, without prior written permission of the publisher.

The work presented in this thesis was funded by the Netherlands Organization for Scientific Research (NWO) and was conducted at Maastricht University.

Cover Gesa Lange

Production CPI Wöhrmann Print Services B. V.

ISBN 978-94-6203-987-2 


\title{
Psychophysical investigations of perceptual learning and attention
}

New insights into old principles

\author{
Dissertation
}

To obtain the degree of Doctor at

Maastricht University,

on the authority of the Rector Magnificus, Prof.dr. L.L.G Soete in accordance with the decision of the Board of Deans,

to be defended in public

on Friday 26 February 2016, at 10.00 hours

by

Gesa Friederike Lange 


\section{Supervisor:}

Prof. dr. Peter De Weerd

\section{Co-supervisor:}

Dr. Mark J. Roberts

Assessment committee:

Prof. dr. Alexander T. Sack (chair)

Prof. dr. Julien Doyon (University of Montreal, Canada)

Prof. dr. Avi Karni (University of Haifa, Israel)

Dr. Judith Peters

Dr. Vincent van de Ven 


\section{CONTENTS}

CHAPTER 1 General Introduction

7

CHAPTER 2 Training in visual orientation discrimination yields

limited position and orientation specificity after

asymptotic learning

CHAPTER 3 Limited transfer of orientation discrimination

71

performance after pre-test and masked exposure

CHAPTER 4 Long-term successive training causes behavioral

99 interference

CHAPTER 5 Interference with visual skill learning in humans by exposure to masked, unattended stimuli

CHAPTER 6 The attentional blink effect is related to microsaccade 189 suppression but not rebound

CHAPTER 7 Summary and Conclusions

Knowledge Valorization

Acknowledgments

Curriculum Vitae

Publications 



\section{Chapter 1}

General Introduction 
Skills are an integral part of our daily lives. Some skills have become so automated over years of practice that we do not have to think about how to perform them, like walking, speaking, or cycling. Other skills that are less essential to our daily functioning do require more conscious effort. A person aspiring to become a birdwatcher, for example, has to acquire many different perceptual skills in order to pursue his interest. Among other skills he has to learn focusing binoculars manually, localizing and distinguishing bird sounds auditorily, discerning a bird from its natural surroundings such as trees and bushes, and using key features to visually discriminate different birds, such as the shape and size of wings and beaks, as well as characteristic coloring of feathers. This still incomplete list illustrates the multitude of skills used by a birdwatcher and the different domains these belong to.

Perceptual learning is conceptually tightly linked with memory and attention. Memory in the context of perceptual learning typically refers to the retention of enhanced performance following training. Attention is an important mechanism for information selection during skill learning, and consequently seen as a pre-requisite for intentional (perceptual) learning. The research presented in this thesis focuses mostly on visual perceptual learning and includes investigations of the contribution of attention and awareness to visual skill. In addition, the research presented also includes one project focusing on the attentional mechanism itself, exploring the relationship between microsaccades and attentional selection. Therefore, the background section will be divided into two parts, one addressing visual perceptual learning, and the other addressing attentional selection mechanisms. For both topics, old principles established by previous empirical work will be presented, questions in current literature regarding these principles discussed, and experimental questions addressed in this thesis derived. Based on these experimental questions, the initial chapters focus on the contributions of low-level visual cortex to visual perceptual leaning (Chapter 2) and on the role of attention and awareness (Chapter 3). The following chapters address questions related to consolidation and interference (Chapter 4) and to the role of attention and awareness in interference (Chapter 5). In a last empirical chapter, a study is presented that focuses on the possible contribution of microsaccades to information selection during attention-demanding 
tasks (Chapter 6). Finally, the findings from the thesis are summarized and what they contribute to the current literature is discussed (Chapter 7).

\section{Visual perceptual learning}

\section{Visual perceptual learning as a form of memory}

To understand the properties of visual perceptual leaning, it is useful to place it in the context of the fundamental differentiation between explicit (or declarative) memory and implicit (or non-declarative) memory. Explicit memory can be consciously recalled, whereas implicit memory refers to the performance of actions and sensory processing not requiring conscious recall. Explicit memory includes episodic memory, related to autobiographic events, and semantic memory, related to factual information, whereas implicit memory is associated with priming, associative learning (conditioning), non-associative learning (habituation and sensitization), and procedural learning (skills and habits). Perceptual learning squarely falls in the latter domain of skill learning (Kandel, Schwartz, Jessell, Siegelbaum, \& Hudspeth, 2012). In addition, all memory formation consists of three component processes, namely encoding/acquisition, storage/consolidation, and retrieval/performance (Kandel et al., 2012). In perceptual skill learning, the daily training corresponds to the encoding/acquisition phase, which is accompanied and followed by a form of consolidation or stabilization of a memory trace, which when retrieved in the trained task leads to enhanced performance.

In this context, perceptual learning can be defined as the acquisition of a perceptual skill (i.e. a memory) through repeated training over time. The training typically requires several weeks, and the resulting learning curve is commonly described as composed of an initial fast learning phase followed by a slower learning phase leading to asymptotic performance levels (Karni \& Sagi, 1993). A remarkable feature of perceptual learning is that a large part of the performance increment takes place in-between sessions while no training is given. When participants start performance in a daily training session, they typically perform better than at the end of the training session of the previous day. This phenomenon is often referred to as 'latent consolidation'. This effect is very large during the beginning few sessions 
and then reduces as learning becomes asymptotic. There are also within-session improvements, but they are typically smaller and usually most noticeable during the beginning phases of learning. Once learned, perceptual skills have been reported to be highly specific for the trained stimuli and motor movements, and they have been reported to remain stable for years (Karni \& Sagi, 1993), which suggests that training induces lasting neural changes and yields a 'consolidated' memory. Many aspects of this view on perceptual learning and skill learning in general have been challenged in recent years.

\section{Different theoretical views on the network processes leading to perceptual learning}

There are two main views on how training leads to a perceptual skill memory. In one view of perceptual learning referred to as the 'lowest-level theory' (for review, see De Weerd, Pinaud, \& Bertini, 2006) it is assumed that the initial, fast improvements in performance are related to setting up rules and strategies in higher hierarchical areas, whereas the subsequent slow learning is related to automatization and possibly long-term structural modifications of early hierarchical areas (Karni \& Sagi, 1993). In many forms of skill learning, it has indeed been established that higher level brain structures involved in working memory and executive function are activated during early learning (Ahissar \& Hochstein, 1993; Doyon, Penhune, \& Ungerleider, 2003; Hochstein \& Ahissar, 2002; Willingham, 1998), of which most decrease their activation during asymptotic learning (Kelly \& Garavan, 2005; Poldrack \& Gabrieli, 2001). In addition, in a number of studies in the somatosensory cortex, using training in simple sensory-motor tasks, topographic representations have been shown to expand (e.g. Furmanski, Schluppeck, \& Engel, 2004; Karni et al., 1995; Nudo, Milliken, Jenkins, \& Merzenich, 1996; Schwartz, Maquet, \& Frith, 2002; Vaina, Belliveau, Des Roziers, \& Zeffiro, 1998). In the visual system, training has not been reported to change topographic representations in low-level areas, but in detection tasks increases in responsivity have been reported (Furmanski, Schluppeck, \& Engel, 2004), while in discrimination tasks decreases in responsivity have been reported (Schiltz et al., 1999; Schiltz, Bodart, Michel, \& Crommelinck, 2001) as well as changes in tuning (Schoups, Vogels, Qian, \& Orban, 
2001). Hence, although the specific changes in low-level areas may depend both on the sensory system and on the task used, overall the data are consistent with a contribution of plasticity in low-level areas to practice-induced increases in perceptual performance, as predicted by the 'lowest-level' theory of perceptual learning (Karni, 1996).

However, in an opposite view, the 'reverse hierarchy' hypothesis posits that perceptual skill learning is not related to plastic changes in low-level sensory areas, but rather with an increased efficiency of reading out sensory information. Moreover, it is suggested that during initial training phases, memory formation takes place predominantly at higher hierarchical levels, and that only when the system is pushed to its limits, information is read out at increasingly lower hierarchical levels where more detailed sensory information is present (Ahissar \& Hochstein, 1997, 2004; Hochstein \& Ahissar, 2002). The importance of read-out has been demonstrated in elegant neurophysiological studies by Law and Gold (2008). They showed that monkeys trained in a difficult motion direction discrimination task did not show any changes in MT, but showed changes in neural processing in LIP with advancing training. With training, LIP neurons preparing the motor response became more efficient in reading out sensory information and using it to build up activity to initiate a motor response (a saccade). This shows, in this particular task, a large contribution of read-out, and no changes in the sensory areas (MT) where low-level changes would be most expected given the task.

The lowest-level and reverse hierarchy theories are conceptually different in the sense that one emphasizes plasticity in low-level sensory areas, whereas the other emphasizes the role of read-out from lower-level sensory areas by higher-level areas at the interface between input and motor output. However, the two theories also share important aspects. First, in both theories the stimulus used will play a large role in determining at which hierarchical level in the sensory system most plasticity is expected (lowest-level theory) or at which hierarchical level read-out will occur. Second, both theoretical frameworks suggest that low-level sensory areas will start playing a role only with training in later stages of the training, which is when the effect of training becomes specific for the trained stimulus. Third, selective attention is accepted to be an important component driving and guiding plastic changes 
associated with low-level reconfigurations or enhanced read-out. It is likely that both enhanced read-out (which implies attention and feedback) and plasticity in low-level areas play a role in perceptual learning, but a systematic investigation of the two processes to perceptual learning is lacking at present. Fourth, both theories predict stimulus and position specificity after extensive perceptual learning. According to lowest-level theory, the specificity of a visual skill for the stimulus used (e.g., orientation of a grating) and for the visual field position in which the skill was trained, emerges during asymptotic learning (e.g., Karni \& Sagi, 1991; Schoups, Vogels, \& Orban, 1995), and is thought to reflect the recruitment of specific subpopulations in the retinotopic map of early visual areas such as V1 and V2 during maximization of performance (Gattass, Gross, \& Sandell, 1981; Hubel, Wiesel, \& Stryker, 1978; Schoups et al., 1995). When extensive training with a specific stimulus in a specific location is followed by a test with a stimulus at a different orientation or position, performance has been reported to generalize very little (Schoups et al., 1995; Schoups et al., 2001). This type of specificity of visual perceptual learning is considered a hallmark of this type of learning (Karni \& Bertini, 1997). However, based on behavioral data alone, it is difficult to ascertain that these effects are not due to enhanced readout of populations relevant for the task. In general, it is striking that two theories that are conceptually so different in essence yield the same predictions. In purely psychophysical studies of perceptual learning, it is therefore difficult to distinguish the contributions of read-out and low level plasticity.

Nevertheless, there is one specific psychophysical design in which the contribution of low-level plasticity can be separated from that of read-out. If one could present specific stimuli in a specific location outside awareness and outside the focus of attention and if this exposure phase would lead to an advantage in subsequent perceptual learning with the pre-exposed stimuli, this form of perceptual leaning would be difficult to explain by enhanced read-out. It is more likely that the pre-exposure would lead to bottom-up driven changes in low-level areas supporting subsequent changes in performance. Hence, any learning that occurs without awareness of the stimuli and without attention could be seen as supporting a contribution of low-level plasticity separate from read-out. Such findings have been 
reported in several studies (Godde, Stauffenberg, Spengler, \& Dinse, 2000; Gutnisky, Hansen, Iliescu, \& Dragoi, 2009; Nishina, Seitz, Kawato, \& Watanabe, 2007; Watanabe, Náñez, \& Sasaki, 2001).

Interestingly, in recent literature two aspects of learning that are accepted as established principles or even hallmarks of perceptual skill learning in both theories have been challenged. First, the very idea that extensive training with a stimulus leads to skills specific for that stimulus has been challenged in recent studies. In these studies, limited training or exposure was given in one location followed by more extensive training in another location. At the end of the latter, extensive training, a transfer of expertise was observed to the location with limited training/exposure (Wang, Zhang, Klein, Levi, \& Yu, 2012; Xiao et al., 2008; T. Zhang, Xiao, Klein, Levi, \& Yu, 2010). It is at present unclear how these studies have to be interpreted, and why these studies give results that seem difficult to reconcile with other studies suggesting strong stimulus and location specificity (Ahissar \& Hochstein, 1993, 1996; Ahissar, Laiwand, Kozminsky, \& Hochstein, 1998; Crist, Kapadia, Westheimer, \& Gilbert, 1997; Fahle, Edelman, \& Poggio, 1995; Fiorentini \& Berardi, 1980; Karni \& Sagi, 1991; Schoups et al., 1995). The main suggestion in cited studies (Wang et al., 2012; Xiao et al., 2008; T. Zhang et al., 2010) is that enhanced readout driven by training in one location might generalize to other locations or stimuli to which there has been some exposure during irrelevant training. An important factor in interpreting these studies is that training was halted typically around session 7, and it could be argued that this is insufficient to trigger specificity (e.g. Jeter, Dosher, Liu, \& Lu, 2010). Second, recent evidence suggests that the hypothesis that low-level areas per definition do not play a role in very early stages of skill learning may be incorrect. Some fMRI studies (Schwartz et al., 2002; Yotsumoto, Watanabe, \& Sasaki, 2008) suggest early contributions of V1, and a recent TMS study (De Weerd et al., 2012) has shown that rTMS directed at V1 can interfere with latent consolidation from the very beginning of visual perceptual learning. The two findings together indicate that some of the core principles underlying the formation of skill memories are being challenged in current literature. This has consequences for the manner in which one should view consolidation, which is assumed to underlie the formation of perceptual skill 
memories. The presented ideas and findings suggest that consolidation following perceptual learning is a process of neural plasticity that involves likely a large network that is dynamically activated and updated after each daily training session, and which includes the specific populations stimulated in low-level sensory areas, but is not limited to it.

\section{New views on the concept of consolidation}

Long-term memory consolidation always is associated with neural plasticity, which is controlled by an interaction between stimulation and genomic responses. Neural plasticity refers to changes in the patterns of functional interconnections among neurons in the brain. There are two main forms of long-term synaptic plasticity, long term potentiation (LTP) leading to an increase in synaptic connections (and their strength) and long-term depression (LTD) leading to a decrease in synaptic connections (and their strength) (Kandel et al., 2012; Shinoda, Tanaka, TominagaYoshino, \& Ogura, 2010; Tominaga-Yoshino, Urakubo, Okada, Matsuda, \& Ogura, 2008). Two different types of LTP are commonly being distinguished: Early LTP, lasting 1-3 hours and leading to increased effectivity of pre-existing synaptic connections, and late LTP, resulting in new synaptic connections and much longerlasting effects (Kandel et al., 2012). Long-term LTP is thought to result from the release of neurotransmitters in association with increased $\mathrm{Ca}^{2+}$ influx. This, in turn, activates second messengers, which initiate a biochemical cascade, resulting in gene expression, new protein synthesis and new (and/or stronger) synaptic connections. LTD is thought to involve a similar sequence of events, where increased $\mathrm{Ca}^{2+}$ influx (to a lesser degree than for LTP) results in a reduction of synaptic connections (Kandel et al., 2012). The role of LTD on a behavioral level is thought to facilitate behavioral flexibility, where memory traces associated with previously learned information are weakened when new information is learned (Nicholls et al., 2008). The LTD/LTP effects and related effects of gene expression that modulate synaptic connectivity are plasticity mechanisms at the cellular level underlying memory consolidation. The expression window of late genes involved in synaptic plasticity is typically in the 3-6h range following experience (Caroni, Chowdhury, \& Lahr, 2014; Igaz, Bekinschtein, Vianna, Izquierdo, \& Medina, 2004), which corresponds well 
with the period of latent consolidation. The term cellular consolidation (Dudai, 1996; Dudai \& Morris, 2000) has been used to refer to the consolidation effects that might primarily be linked to these short term genomic effects. However, it has been proposed that in addition to cellular consolidation, there is also systems consolidation (Debiec, LeDoux, \& Nader, 2002; Dudai, 2012). This refers to the fact that multiple structures in the brain will show interactions with each other following learning, such that cellular plasticity in one part of a network could instigate plasticity in another part of a network at a later time. This would suggest the existence of multiple consolidation periods. The idea of multiple consolidation periods has been supported by a number of studies (Caithness et al., 2004; Lee, 2008). This idea is also in agreement with contributions of sleep to consolidation (Walker \& Stickgold, 2005; Yotsumoto et al., 2009). In motor skill learning it has additionally been proposed to distinguish two different components of memory consolidation, namely offline improvement of skills and memory stabilization (Robertson, 2012).

To gain insight in the time course of consolidation, many studies have used an interference approach. The underlying idea is that during consolidation, memory traces should be vulnerable and subject to interference, while memory traces after consolidation should have become much more stable and resistant against interference. In interference studies, the stability of a memory trace (and hence resistance of retention against interference) is tested by training participants on a first task, followed within a number of hours by training on a second task, or by biological manipulations. Findings from studies interfering with learning behaviorally (Been, Jans, \& De Weerd, 2011; Brashers-Krug, Shadmehr, \& Bizzi, 1996; Caithness et al., 2004; Goedert \& Willingham, 2002; Seitz, 2005; Shadmehr \& Holcomb, 1997; J.-Y. Zhang et al., 2008), pharmacologically (Bütefisch et al., 2000; Donchin, Sawaki, Madupu, Cohen, \& Shadmehr, 2002; Sawaki et al., 2002; Thiel, Henson, Morris, Friston, \& Dolan, 2001), or by applying non-invasive brain stimulation techniques (De Weerd et al., 2012; Muellbacher et al., 2002) have all shown that consolidation can be impaired during time intervals that seem compatible with a single or perhaps several cascades of plasticity and gene expression. However, some studies, in which interference has been tested with longer time 
intervals of up to 48h (Been et al., 2011; Caithness et al., 2004; Goedert \& Willingham, 2002) raise doubts about how to interpret interference effects. If the effect of interference is related to instability during memory formation, one has to accept that consolidation processes can take a very long time. This is not totally implausible, but nevertheless, an alternative view has emerged, both in the domain of episodic/declarative memory (Forcato et al., 2007; Hupbach, Gomez, \& Nadel, 2009) and in the domain of skill learning (Walker, Brakefield, Hobson, \& Stickgold, 2003). In this alternative view, it is posited that memory traces never become truly stable but rather exist in active and inactive forms. Crucial in this view is that retrieval of the memory (and performance in case of skill learning) at any time will reactivate the memory trace thereby enabling updates of the memory (as in training) but also allowing in some cases undesirable changes (interference) (Nadel, Hupbach, Gomez, \& Newman-Smith, 2012; Nader \& Einarsson, 2010; Stickgold \& Walker, 2005). In the ideas of Nadel, the continually ongoing processes of consolidation, retrieval, and reconsolidation are emphasized (Nadel \& Land, 2000), whereas in the ideas of Nader, it is emphasized that memories exist in dormant and reactivated form (Nader, 2003; Nader \& Einarsson, 2010; Nader, Schafe, \& LeDoux, 2000). In both theoretical frameworks, it is clear that the original meaning of the concept of consolidation has been thoroughly revised.

Based on the state of the reviewed literature, we identified a number of questions related to visual perceptual learning, which we set out to investigate in the present thesis. In all investigations of visual skill learning, we used an orientation discrimination task with Gabor stimuli. Participants underwent training in orientation discrimination for at least 3 weeks (15 daily sessions) to determine learning curves in one or more conditions. Daily performance was expressed in thresholds, which decreased as a function of learning. We chose this task because enormous knowledge exists on the neurophysiological mechanisms contributing to the learning both in lower (Gattass et al., 1981; Hubel et al., 1978; Raiguel, Lagae, Gulyàs, \& Orban, 1989; Schoups et al., 2001) and higher (Sary, Vogels, Kovacs, \& Orban, 1995; Vogels \& Orban, 1994) hierarchical levels of the visual system. Moreover, this task is one of the tasks around which controversy around the properties and mechanisms of visual perceptual learning has become crystallized. 


\section{Empirical questions related to mechanisms underlying visual skill learning}

As mentioned previously, one characteristic of perceptual learning is its specificity. However, recently empirical evidence has cast doubt on the generality of this old principle, suggesting that exposure or short training with one stimulus in a given location enables generalization to other locations and/or stimuli. This raises questions about the size and location of the neural network involved in visual skill learning and memory formation. Accordingly, the questions underlying the first two empirical chapters (Chapters 2 and 3) relate to the extent of the network contributing to orientation discrimination learning. In particular, we attempted by purely psychophysical designs to address the question to what extent bottom-up input to low-level areas and to what extent awareness and selective attention (and read-out), which also involves higher-level visual areas, contribute to perceptual learning. This is a challenging undertaking, and in the following paragraphs we sketch the manner in which we approached this.

In Chapter 2, we aimed to reinvestigate stimulus specificity in orientation discrimination learning. The extent to which visual perceptual learning is stimulus and location specific is an important question, as a total lack of specificity or the ability of effortless generalization enabled by very limited pre-exposure would require a framework of perceptual learning that is very different from both dominant views in the literature (lowest-level theory and reverse hierarchy theory). Hence, in Chapter 2, we re-investigated orientation and position specificity of orientation discrimination learning. We had two aims in that study. Instead of investigating generalization after training in a single peripheral location along an equi-eccentric line, we investigated generalization along a line of equal polar angle. This allowed us to test whether there were asymmetries in generalization as can be expected from cortical magnification of central vision in early retinotopic visual areas. A second aim was to test the idea that specificity emerges in the asymptotic phase of learning, and that the early phase of learning does not contribute to specificity. Asymmetry in generalization combined with a predominant contribution to specificity from asymptotic learning might be seen as behavioral evidence for a contribution of plasticity in low-level regions to the storage of visual skill. In Chapter 3, we tested the contribution of read-out to orientation discrimination learning using a design in 
which various forms of pre-exposure were applied after which the benefits of preexposure were tested by training in the pre-exposed locations. One aim of the study was to verify whether we could replicate the generalization of skill in a heavily trained location to a location where very limited pre-training took place. In this case, strong generalization would be indicative of a strong contribution of read-out enhancements to skill learning. In other conditions, a substantial amount of preexposure was given, using stimuli of which participants were not aware, and it was tested whether this led to a significant benefit for subsequent training. If this were the case, it would point to a substantial contribution of local plasticity limited to low level areas such as V1 and V2, merely triggered by exposure (and unlikely to be attributed to readout).

The broad question underlying the next two empirical chapters (Chapters 4 and 5) relates to the nature of the process that leads to the stabilization of visual skill memory. The concept of consolidation is generally regarded as useful for understanding perceptual learning and interference thereof. However, recent findings of behavioral interference after time intervals of up to 48 hours question the universality of the consolidation principle.

Chapter 4 was inspired by the study of Been et al. (2011), which showed that orientation discrimination training at an oblique reference orientation strongly suffered from subsequent training at other nearby reference orientations. This interference disappeared when the reference orientations used for training were orthogonal. However, the interference did not disappear by increasing the delay between the first and second tasks, for intervals up to $24 \mathrm{~h}$. In Chapter 4 , we aimed to train one orientation discrimination task for three weeks before switching to the next training period of three weeks with another task, to see whether interference would bridge time periods as long as three weeks. If this were the case, it would add weight to the interpretation of skill memories as permanently malleable, and question the classical concept of consolidation. In addition, based on an already existing model by Teich and Qian (2003), we aimed to devise a network model that would be able to replicate the rate of learning in control learning and the level of interference among competing tasks, and use this model to evaluate the likelihood that a classical consolidation mechanism would underlie the empirical data. In Chapter 5, we aimed 
to test whether extensive exposure outside awareness could not only contribute to learning, but also to interference. The sensitivity for interference that will be demonstrated in Chapter 4 demonstrates the possibility that under specific conditions forms of stimulation much more subtle than performance of a competing task may still lead to interference.

\section{Visual attention and microsaccades}

Although a number of studies have shown that perceptual learning can take place in the absence of perception, and in the absence of attention (Gutnisky et al., 2009; Watanabe et al., 2001), it is also true that with attention visual skill learning is more effective than without (e.g., Gutnisky et al., 2009). Visual attention is a crucial aspect in the selection of target stimuli from other objects in the environment (e.g. Carrasco, 2011; Desimone \& Duncan, 1995; Duncan, 1984), and in the selection of relevant dimensions within the stimulus (e.g., Maunsell \& Treue, 2006; Motter, 1994; Serences \& Boynton, 2007). In both read-out theories and lowest-level theories, the allocation of attention is hypothesized to change in the course of learning, with full expertise corresponding to an increased automatization of sensory processing relevant for the skill.

The pre-motor theory of attention (Rizzolatti, Riggio, Dascola, \& Umiltá, 1987), which is very influential but not less controversial, posits that the same structures are involved in the planning of eye-movements and directing of attention, and that shifting attention basically is equal to programming (but not necessarily executing) an eye movement. Over the decades there has been quite some controversy regarding this theory and there is some well-grounded empirical evidence that it should be rejected for endogenous attention though it still might hold for exogenous attention (for a review see Smith \& Schenk, 2012). Though the initial idea that planning of eye movements is equivalent to attentional allocation has been refuted, there has been tremendous interest in the role of microsaccades, one type of miniature eye movements, in perception and attention (Martinez-Conde, Macknik, \& Hubel, 2004; Rolfs, 2009). In the context of perceptual learning, it could be expected that patterns of microsaccades change when sensory processing becomes more efficient and automated during learning. 
Microsaccades are jerk-like eye movements that occur during voluntary fixation with a frequency of 1-4 Hz (e.g. Bosman, Womelsdorf, Desimone, \& Fries, 2009; Engbert \& Kliegl, 2003). They are thought, among other things, to prevent retinal fading of an image due to adaptation and to correct for fixational error (MartinezConde et al., 2004). Though microsaccades are crucial for stability of perception, the movement itself can also hamper perception (Hafed, 2013; Schütz, Braun, \& Gegenfurtner, 2009), so suppression of microsaccades during demanding tasks can actually prove advantageous (Bridgeman \& Palca, 1980). Both visual input (Cui, Wilke, Logothetis, Leopold, \& Liang, 2009; Gowen, Abadi, \& Poliakoff, 2005; Hsieh \& Tse, 2009) and cognitive factors (Betta \& Turatto, 2006; Gowen et al., 2005; Kohama, Endoh, \& Yoshida, 2013) have an influence on microsaccade rate. The onset of a stimulus has been shown to reliably induce a so called microsaccade rate signature, an initial rapid reduction in MS rate (100-500ms), with a later rebound (200-600ms) (Betta \& Turatto, 2006; Hafed \& Ignashchenkova, 2013; Rolfs, 2009; Rolfs, Engbert, \& Kliegl, 2005; Rolfs, Kliegl, \& Engbert, 2008). During states of sustained attention a reduced microsaccade rate is observed and has been associated with a direction bias towards (e.g. Laubrock, Engbert, \& Kliegl, 2005; Laubrock, Kliegl, Rolfs, \& Engbert, 2010; Yuval-Greenberg, Merriam, \& Heeger, 2014) or away (e.g. Galfano, Betta, \& Turatto, 2004; Rolfs et al., 2005; Tse, Sheinberg, \& Logothetis, 2002) from the attended location. These findings demonstrate a close link between microsaccades and attention.

Although an investigation of changes in microsaccade behaviour as a function of visual skill learning would be highly interesting (see Otto, Herzog, Fahle, \& Zhaoping, 2006; Zhaoping, Herzog, \& Dayan, 2003), we decided to test the link between attention and microsaccades in the context of the attentional blink (AB) paradigm. This paradigm allows one to look into the temporal aspect of attention allocation (Shapiro, Raymond, \& Arnell, 1997). In this experimental paradigm, participants are shown a rapid stream of distracter letters among which they have to detect two target letters, referred to as T1 and T2. The temporal delay between T1 and T2 strongly influences the percentage of T2 detection. Around 200ms delay between $\mathrm{T} 1$ and $\mathrm{T} 2$ a strong decrease in $\mathrm{T} 2$ detection performance is commonly observed. The processing resources required by the $\mathrm{AB}$ paradigm include encoding, 
working memory, response selection and most importantly attention. So an interesting question is what happens when attention lacks and the $\mathrm{AB}$ effect occurs.

Empirical questions about the links between attention, microsaccades, and the attentional blink effect

Though there is disagreement about the mechanism behind the attentional blink phenomenon (for a review, see Martens \& Wyble, 2010), most theories attribute the effect to a late processing stage and not to sensory deficits (e.g. Shapiro et al., 1997). The old principle that the attentional blink is in fact related to attentional or memory-related processes was investigated by exploring the possibility that microsaccades contribute to the well-known attentional blink phenomenon.

We hypothesized that microsaccades when occurring close to or during the presentation of $\mathrm{T} 2$ would lead to reduced performance in detecting $\mathrm{T} 2$ when presented $200 \mathrm{~ms}$ after $\mathrm{T} 1$, an effect referred to as the attentional blink. We also asked the question whether attention as an intermediary construct is necessary, and whether the attentional blink in fact could be explained as a pure effect of microsaccades on the visibility of T2. These questions were investigated in Chapter 6. In the General Discussion (Chapter 7), findings from all chapters will be integrated and evaluated in the context of the current literature. 


\section{References}

Ahissar, M., \& Hochstein, S. (1993). Attentional control of early perceptual learning. Proceedings of the National Academy of Sciences, 90(12), 5718-5722.

Ahissar, M., \& Hochstein, S. (1996). Learning pop-out detection: Specificities to stimulus characteristics. Vision Research, 36(21), 3487-3500.

Ahissar, M., \& Hochstein, S. (1997). Task difficulty and the specificity of perceptual learning. Nature, 387(6631), 401-406.

Ahissar, M., \& Hochstein, S. (2004). The reverse hierarchy theory of visual perceptual learning. Trends in Cognitive Sciences, 8(10), 457-464.

Ahissar, M., Laiwand, R., Kozminsky, G., \& Hochstein, S. (1998). Learning pop-out detection: Building representations for conflicting target-distractor relationships. Vision Research, 38(20), 3095-3107.

Been, M., Jans, B., \& De Weerd, P. (2011). Time-limited consolidation and task interference: No direct link. The Journal of Neuroscience, 31(42), 14944-14951.

Betta, E., \& Turatto, M. (2006). Are you ready? I can tell by looking at your microsaccades. Neuroreport, 17(10), 1001-1004.

Bosman, C. A., Womelsdorf, T., Desimone, R., \& Fries, P. (2009). A microsaccadic rhythm modulates gamma-band synchronization and behavior. The Journal of Neuroscience, 29(30), 9471-9480.

Brashers-Krug, T., Shadmehr, R., \& Bizzi, E. (1996). Consolidation in human motor memory. Nature, 382(6588), 252-255.

Bridgeman, B., \& Palca, J. (1980). The role of microsaccades in high acuity observational tasks. Vision Research, 20(9), 813-817.

Bütefisch, C. M., Davis, B. C., Wise, S. P., Sawaki, L., Kopylev, L., Classen, J., \& Cohen, L. G. (2000). Mechanisms of use-dependent plasticity in the human motor cortex. Proceedings of the National Academy of Sciences, 97(7), 3661-3665.

Caithness, G., Osu, R., Bays, P., Chase, H., Klassen, J., Kawato, M., . . Flanagan, J. R. (2004). Failure to consolidate the consolidation theory of learning for sensorimotor adaptation tasks. The Journal of Neuroscience, 24(40), 8662-8671.

Caroni, P., Chowdhury, A., \& Lahr, M. (2014). Synapse rearrangements upon learning: From divergent-sparse connectivity to dedicated sub-circuits. Trends in Neurosciences, 37(10), 604-614. 
Carrasco, M. (2011). Visual attention: The past 25 years. Vision Research, 51(13), 1484-1525.

Crist, R. E., Kapadia, M. K., Westheimer, G., \& Gilbert, C. D. (1997). Perceptual learning of spatial localization: Specificity for orientation, position, and context. Journal of Neurophysiology, 78(6), 2889-2894.

Cui, J., Wilke, M., Logothetis, N. K., Leopold, D. A., \& Liang, H. (2009). Visibility states modulate microsaccade rate and direction. Vision Research, 49(2), 228-236.

De Weerd, P., Pinaud, R., \& Bertini, G. (2006). Plasticity in v1 induced by perceptual learning. In R. Pinaud, L. Tremere \& P. De Weerd (Eds.), Plasticity in the visual system (pp. 245-283): Springer US.

De Weerd, P., Reithler, J., van de Ven, V., Been, M., Jacobs, C., \& Sack, A. T. (2012). Posttraining transcranial magnetic stimulation of striate cortex disrupts consolidation early in visual skill learning. The Journal of Neuroscience,32(6), 1981-1988.

Debiec, J., LeDoux, J. E., \& Nader, K. (2002). Cellular and systems reconsolidation in the hippocampus. Neuron, 36(3), 527-538.

Desimone, R., \& Duncan, J. (1995). Neural mechanisms of selective visual attention. Annual Review of Neuroscience, 18(1), 193-222.

Donchin, O., Sawaki, L., Madupu, G., Cohen, L. G., \& Shadmehr, R. (2002). Mechanisms influencing acquisition and recall of motor memories. Journal of Neurophysiology, 88(4), 2114-2123.

Doyon, J., Penhune, V., \& Ungerleider, L. G. (2003). Distinct contribution of the cortico-striatal and cortico-cerebellar systems to motor skill learning. Neuropsychologia, 41(3), 252-262.

Dudai, Y. (1996). Consolidation: Fragility on the road to the engram. Neuron, 17(3), 367-370.

Dudai, Y. (2012). The restless engram: Consolidations never end. Annual Review of Neuroscience, 35(1), 227-247.

Dudai, Y., \& Morris, R. G. (2000). To consolidate or not to consolidate: What are the questions. Brain, perception, memory. Advances in cognitive sciences, 149-162.

Duncan, J. (1984). Selective attention and the organization of visual information. Journal of Experimental Psychology: General, 113(4), 501.

Engbert, R., \& Kliegl, R. (2003). Microsaccades uncover the orientation of covert attention. Vision Research, 43(9), 1035-1045. 
Fahle, M., Edelman, S., \& Poggio, T. (1995). Fast perceptual learning in hyperacuity. Vision Research, 35(21), 3003-3013.

Fiorentini, A., \& Berardi, N. (1980). Perceptual learning specific for orientation and spatial frequency. Nature, 287(5777), 43-44.

Forcato, C., Burgos, V. L., Argibay, P. F., Molina, V. A., Pedreira, M. E., \& Maldonado, H. (2007). Reconsolidation of declarative memory in humans. Learning \& Memory, 14(4), 295-303.

Furmanski, C. S., Schluppeck, D., \& Engel, S. A. (2004). Learning strengthens the response of primary visual cortex to simple patterns. Current Biology, 14(7), 573578.

Galfano, G., Betta, E., \& Turatto, M. (2004). Inhibition of return in microsaccades. Experimental Brain Research, 159(3), 400-404.

Gattass, R., Gross, C., \& Sandell, J. (1981). Visual topography of v2 in the macaque. Journal of Comparative Neurology, 201(4), 519-539.

Godde, B., Stauffenberg, B., Spengler, F., \& Dinse, H. R. (2000). Tactile coactivation-induced changes in spatial discrimination performance. The Journal of Neuroscience, 20(4), 1597-1604.

Goedert, K. M., \& Willingham, D. B. (2002). Patterns of interference in sequence learning and prism adaptation inconsistent with the consolidation hypothesis. Learning \& Memory, 9(5), 279-292.

Gowen, E., Abadi, R. V., \& Poliakoff, E. (2005). Paying attention to saccadic intrusions. Brain Res Cogn Brain Res, 25(3), 810-825.

Gutnisky, D. A., Hansen, B. J., Iliescu, B. F., \& Dragoi, V. (2009). Attention alters visual plasticity during exposure-based learning. Current Biology, 19(7), 555- 560.

Hafed, Z. M. (2013). Alteration of visual perception prior to microsaccades. Neuron, 77(4), 775-786.

Hafed, Z. M., \& Ignashchenkova, A. (2013). On the dissociation between microsaccade rate and direction after peripheral cues: Microsaccadic inhibition revisited. The Journal of Neuroscience, 33(41), 16220-16235.

Hochstein, S., \& Ahissar, M. (2002). View from the top: Hierarchies and reverse hierarchies in the visual system. Neuron, 36(5), 791-804.

Hsieh, P. J., \& Tse, P. U. (2009). Microsaccade rate varies with subjective visibility during motion-induced blindness. PLoS One, 4(4), e5163. 
Hubel, D. H., Wiesel, T. N., \& Stryker, M. P. (1978). Anatomical demonstration of orientation columns in macaque monkey. The Journal of Comparative Neurology, 177(3), 361-379.

Hupbach, A., Gomez, R., \& Nadel, L. (2009). Episodic memory reconsolidation: Updating or source confusion? Memory, 17(5), 502-510.

Igaz, L., Bekinschtein, P., Vianna, M. R., Izquierdo, I., \& Medina, J. (2004). Gene expression during memory formation. Neurotoxicity Research, 6(3), 189-203.

Jeter, P. E., Dosher, B. A., Liu, S.-H., \& Lu, Z.-L. (2010). Specificity of perceptual learning increases with increased training. Vision Research, 50(19), 1928-1940.

Kandel, E. R., Schwartz, J. H., Jessell, T. M., Siegelbaum, S. A., \& Hudspeth, A. (2012). Principles of neural science (5 ed.): McGraw-Hill New York.

Karni, A. (1996). The acquisition of perceptual and motor skills: A memory system in the adult human cortex. Cognitive Brain Research, 5(1-2), 39-48.

Karni, A., \& Bertini, G. (1997). Learning perceptual skills: Behavioral probes into adult cortical plasticity. Current Opinion in Neurobiology, 7(4), 530-535.

Karni, A., Meyer, G., Jezzard, P., Adams, M. M., Robert, T., \& Ungerleider, L. G. (1995). Functional MRI evidence for adult motor cortex plasticity during motor skill learning. Nature, 377, 155-158.

Karni, A., \& Sagi, D. (1991). Where practice makes perfect in texture discrimination: Evidence for primary visual cortex plasticity. Proceedings of the National Academy of Sciences, 88(11), 4966-4970.

Karni, A., \& Sagi, D. (1993). The time course of learning a visual skill. Nature, 365(6443), 250-252.

Kelly, A. C., \& Garavan, H. (2005). Human functional neuroimaging of brain changes associated with practice. Cerebral Cortex, 15(8), 1089-1102.

Kohama, T., Endoh, S., \& Yoshida, H. (2013). Persistent inhibition of microsaccades caused by attentional concentration. Perception, 42(ECVP Abstract Supplement), 38-38.

Laubrock, J., Engbert, R., \& Kliegl, R. (2005). Microsaccade dynamics during covert attention. Vision Research, 45(6), 721-730.

Laubrock, J., Kliegl, R., Rolfs, M., \& Engbert, R. (2010). When do microsaccades follow spatial attention? Attention, Perception, \& Psychophysics, 72(3), 683- 694.

Law, C.-T., \& Gold, J. I. (2008). Neural correlates of perceptual learning in a sensory-motor, but not a sensory, cortical area. Nat Neurosci, 11(4), 505-513. 
Lee, J. L. (2008). Memory reconsolidation mediates the strengthening of memories by additional learning. Nature neuroscience, 11(11), 1264.

Martens, S., \& Wyble, B. (2010). The attentional blink: Past, present, and future of a blind spot in perceptual awareness. Neuroscience \& Biobehavioral Reviews, 34(6), 947-957.

Martinez-Conde, S., Macknik, S. L., \& Hubel, D. H. (2004). The role of fixational eye movements in visual perception. Nature Reviews Neuroscience, 5(3), 229- 240.

Maunsell, J. H. R., \& Treue, S. (2006). Feature-based attention in visual cortex. Trends in Neurosciences, 29(6), 317-322.

Motter, B. C. (1994). Neural correlates of feature selective memory and pop-out in extrastriate area v4. The Journal of Neuroscience, 14(4), 2190-2199.

Nadel, L., Hupbach, A., Gomez, R., \& Newman-Smith, K. (2012). Memory formation, consolidation and transformation. Neuroscience \& Biobehavioral Reviews, 36(7), 1640-1645.

Nadel, L., \& Land, C. (2000). Commentary [mdash] reconsolidation : Memory traces revisited. Nat Rev Neurosci, 1(3), 209-212.

Nader, K. (2003). Memory traces unbound. Trends in Neurosciences, 26(2), 65-72.

Nader, K., \& Einarsson, E. Ö. (2010). Memory reconsolidation: An update. Annals of the New York Academy of Sciences, 1191(1), 27-41.

Nader, K., Schafe, G. E., \& LeDoux, J. E. (2000). Reply-reconsolidation: The labile nature of consolidation theory. Nature Reviews Neuroscience, 1(3), 216- 219.

Nicholls, R. E., Alarcon, J. M., Malleret, G., Carroll, R. C., Grody, M., Vronskaya, S., \& Kandel, E. R. (2008). Transgenic mice lacking nmdar-dependent ltd exhibit deficits in behavioral flexibility. Neuron, 58(1), 104-117.

Nishina, S., Seitz, A. R., Kawato, M., \& Watanabe, T. (2007). Effect of spatial distance to the task stimulus on task-irrelevant perceptual learning of static gabors. Journal of Vision, 7(13).

Nudo, R. J., Milliken, G., Jenkins, W. M., \& Merzenich, M. M. (1996). Usedependent alterations of movement representations in primary motor cortex of adult squirrel monkeys. Journal of Neuroscience, 16(2), 785-807.

Otto, T. U., Herzog, M. H., Fahle, M., \& Zhaoping, L. (2006). Perceptual learning with spatial uncertainties. Vision Research, 46(19), 3223-3233. 
Poldrack, R. A., \& Gabrieli, J. D. E. (2001). Characterizing the neural mechanisms of skill learning and repetition priming (Vol. 124).

Raiguel, S. E., Lagae, L., Gulyàs, B., \& Orban, G. A. (1989). Response latencies of visual cells in macaque areas v1, v2 and v5. Brain Research, 493(1), 155-159.

Rizzolatti, G., Riggio, L., Dascola, I., \& Umiltá, C. (1987). Reorienting attention across the horizontal and vertical meridians: Evidence in favor of a premotor theory of attention. Neuropsychologia, 25(1), 31-40.

Robertson, Edwin M. (2012). New insights in human memory interference and consolidation. Current Biology, 22(2), R66-R71.

Rolfs, M. (2009). Microsaccades: Small steps on a long way. Vision Research, 49(20), 2415-2441.

Rolfs, M., Engbert, R., \& Kliegl, R. (2005). Crossmodal coupling of oculomotor control and spatial attention in vision and audition. Experimental Brain Research, 166(3-4), 427-439.

Rolfs, M., Kliegl, R., \& Engbert, R. (2008). Toward a model of microsaccade generation: The case of microsaccadic inhibition. Journal of Vision, 8(11), 5 1-23.

Sary, G., Vogels, R., Kovacs, G., \& Orban, G. A. (1995). Responses of monkey inferior temporal neurons to luminance-, motion-, and texture-defined gratings (Vol. 73).

Sawaki, L., Boroojerdi, B., Kaelin-Lang, A., Burstein, A. H., Bütefisch, C. M., Kopylev, L., . . . Cohen, L. G. (2002). Cholinergic influences on use-dependent plasticity. Journal of Neurophysiology, 87(1), 166-171.

Schiltz, C., Bodart, J. M., Dubois, S., Dejardin, S., Michel, C., Roucoux, A., . . . Orban, G. A. (1999). Neuronal mechanisms of perceptual learning: Changes in human brain activity with training in orientation discrimination. NeuroImage, 9(1), 46-62.

Schiltz, C., Bodart, J. M., Michel, C., \& Crommelinck, M. (2001). A pet study of human skill learning: Changes in brain activity related to learning an orientation discrimination task. Cortex, 37(2), 243-265.

Schoups, A. A., Vogels, R., \& Orban, G. A. (1995). Human perceptual learning in identifying the oblique orientation: Retinotopy, orientation specificity and monocularity. Journal of Physiology, 483(Pt 3), 797-810.

Schoups, A. A., Vogels, R., Qian, N., \& Orban, G. A. (2001). Practising orientation identification improves orientation coding in v1 neurons. Nature, 412(6846), 549553. 
Schütz, A. C., Braun, D. I., \& Gegenfurtner, K. R. (2009). Object recognition during foveating eye movements. Vision Research, 49(18), 2241-2253.

Schwartz, S., Maquet, P., \& Frith, C. (2002). Neural correlates of perceptual learning: A functional mri study of visual texture discrimination. Proceedings of the National Academy of Sciences, 99(26), 17137-17142.

Seitz, A. R. (2005). Task-specific disruption of perceptual learning. Proceedings of the National Academy of Sciences, 102(41), 14895-14900.

Serences, J. T., \& Boynton, G. M. (2007). Feature-based attentional modulations in the absence of direct visual stimulation. Neuron, 55(2), 301-312.

Shadmehr, R., \& Holcomb, H. H. (1997). Neural correlates of motor memory consolidation. Science, 277(5327), 821-825.

Shapiro, K. L., Raymond, J., \& Arnell, K. (1997). The attentional blink. Trends in Cognitive Sciences, 1(8), 291-296.

Shinoda, Y., Tanaka, T., Tominaga-Yoshino, K., \& Ogura, A. (2010). Persistent synapse loss induced by repetitive ltd in developing rat hippocampal neurons. PLoS One, 5(4), e10390.

Smith, D. T., \& Schenk, T. (2012). The premotor theory of attention: Time to move on? Neuropsychologia, 50(6), 1104-1114.

Stickgold, R., \& Walker, M. P. (2005). Memory consolidation and reconsolidation: What is the role of sleep? Trends in Neurosciences, 28(8), 408-415.

Teich, A. F., \& Qian, N. (2003). Learning and adaptation in a recurrent model of v1 orientation selectivity. Journal of Neurophysiology, 89(4), 2086-2100.

Thiel, C. M., Henson, R. N. A., Morris, J. S., Friston, K. J., \& Dolan, R. J. (2001). Pharmacological modulation of behavioral and neuronal correlates of repetition priming. The Journal of Neuroscience, 21(17), 6846-6852.

Tominaga-Yoshino, K., Urakubo, T., Okada, M., Matsuda, H., \& Ogura, A. (2008). Repetitive induction of late-phase ltp produces long-lasting synaptic enhancement accompanied by synaptogenesis in cultured hippocampal slices. Hippocampus, 18(3), 281-293.

Tse, P. U., Sheinberg, D. L., \& Logothetis, N. K. (2002). Fixational eye movements are not affected by abrupt onsets that capture attention. Vision Research, 42(13), 1663-1669. 
Vaina, L. M., Belliveau, J. W., Des Roziers, E. B., \& Zeffiro, T. A. (1998). Neural systems underlying learning and representation of global motion. Proceedings of the National Academy of Sciences, 95(21), 12657-12662.

Vogels, R., \& Orban, G. A. (1994). Activity of inferior temporal neurons during orientation discrimination with successively presented gratings (Vol. 71).

Walker, M. P., Brakefield, T., Hobson, J. A., \& Stickgold, R. (2003). Dissociable stages of human memory consolidation and reconsolidation. Nature, 425(6958), 616-620.

Walker, M. P., \& Stickgold, R. (2005). It's practice, with sleep, that makes perfect: Implications of sleep-dependent learning and plasticity for skill performance. Clinics in Sports Medicine, 24(2), 301-317.

Wang, R., Zhang, J.-Y., Klein, S. A., Levi, D. M., \& Yu, C. (2012). Task relevancy and demand modulate double-training enabled transfer of perceptual learning. Vision Research, 61(0), 33-38.

Watanabe, T., Náñez, J. E., \& Sasaki, Y. (2001). Perceptual learning without perception. Nature, 413(6858), 844-848.

Willingham, D. B. (1998). A neuropsychological theory of motor skill learning. Psychological review, 105(3), 558.

Xiao, L.-Q., Zhang, J.-Y., Wang, R., Klein, S. A., Levi, D. M., \& Yu, C. (2008). Complete transfer of perceptual learning across retinal locations enabled by double training. Current Biology, 18(24), 1922-1926.

Yotsumoto, Y., Sasaki, Y., Chan, P., Vasios, C. E., Bonmassar, G., Ito, N., . . . Watanabe, T. (2009). Location-specific cortical activation changes during sleep after training for perceptual learning. Current Biology, 19(15), 1278-1282.

Yotsumoto, Y., Watanabe, T., \& Sasaki, Y. (2008). Different dynamics of performance and brain activation in the time course of perceptual learning. Neuron, 57(6), 827-833.

Yuval-Greenberg, S., Merriam, E. P., \& Heeger, D. J. (2014). Spontaneous microsaccades reflect shifts in covert attention. The Journal of Neuroscience, 34(41), 13693-13700.

Zhang, J.-Y., Kuai, S.-G., Xiao, L.-Q., Klein, S. A., Levi, D. M., \& Yu, C. (2008). Stimulus coding rules for perceptual learning. PLoS Biology, 6(8), e197.

Zhang, T., Xiao, L.-Q., Klein, S. A., Levi, D. M., \& Yu, C. (2010). Decoupling location specificity from perceptual learning of orientation discrimination. Vision Research, 50(4), 368-374. 
Zhaoping, L., Herzog, M. H., \& Dayan, P. (2003). Nonlinear ideal observation and recurrent preprocessing in perceptual learning. Network: Computation in Neural Systems, 14(2), 233-247. 


\section{CHAPTER 2}

Training in visual orientation discrimination yields limited position and orientation specificity after asymptotic learning

Lange, G., Lowet, E., Roberts, M. J., \& De Weerd, P. (in preparation). Training in visual orientation discrimination yields limited position and orientation specificity after asymptotic learning. 


\begin{abstract}
There is current debate about the specificity of human orientation discrimination learning. Here, we investigated whether 15 sessions of orientation discrimination training around an oblique reference orientation of $135^{\circ}$ at a single position (at $6^{\circ}$ eccentricity along a $135^{\circ}$ polar angle line) would transfer to test positions along the same line of polar angle (at $3^{\circ}, 9^{\circ}, 12^{\circ}$, and $15^{\circ}$ eccentricity) and to a test orientation $\left(45^{\circ}\right)$ orthogonal to the trained orientation. After completion of training, thresholds at the $135^{\circ}$ reference in the trained position were consistently smaller compared to neighboring test positions. Likewise, orientation discrimination tested in the trained position yielded thresholds at the trained $135^{\circ}$ reference smaller than those obtained at the untrained $45^{\circ}$ reference orientation. This advantage of the trained over the untrained reference was to a lesser extent also present in test positions other than the trained position. Position and orientation specificity were greatest for participants who had continued to show threshold decreases during the last five sessions of training. By contrast, the magnitude of performance improvements in the first five sessions was unrelated to position or orientation specificity. We also found a weak trend for skill to spread more towards peripheral than to central positions relative to the trained position, which may indicate contributions from low-level areas characterized by cortical magnification. In summary, our study supports the concept of specificity in perceptual learning, and shows that perceptual skills become specific only during asymptotic learning. These data provide new insights into mechanisms of perceptual learning, specificity, and the lack thereof in specific conditions.
\end{abstract}




\section{Introduction}

Previous research has emphasized the specificity of visual skill learning. Training induced performance enhancements have been found to be specific for the trained eye (Karni \& Sagi, 1991), retinotopic position (M. Ahissar \& Hochstein, 1996; Crist, Kapadia, Westheimer, \& Gilbert, 1997; Fahle, Edelman, \& Poggio, 1995; Karni \& Sagi, 1991; Aniek A. Schoups, Rufin Vogels, \& Guy A. Orban, 1995), direction (Ball \& Sekuler, 1981; Watanabe et al., 2002), orientation (M. Ahissar \& Hochstein, 1993, 1996; M. Ahissar, Laiwand, Kozminsky, \& Hochstein, 1998; Crist et al., 1997; Fiorentini \& Berardi, 1980; Karni \& Sagi, 1991; Schoups, Vogels, Qian, \& Orban, 2001), spatial frequency (Fiorentini \& Berardi, 1980), stimulus size (M. Ahissar \& Hochstein, 1993, 1996), and the requirements of the task (M. Ahissar \& Hochstein, 1993; Fahle et al., 1995). While such specificity has been taken as evidence for low-level plasticity through perceptual learning (e.g., Crist et al., 1997; Karni \& Bertini, 1997; Karni \& Sagi, 1993), this view has been challenged by studies that demonstrated transfer for the above mentioned low-level stimulus characteristics (Aberg, Tartaglia, \& Herzog, 2009; Ghose, Yang, \& Maunsell, 2002; Jeter, Dosher, Liu, \& Lu, 2010; Jeter, Dosher, Petrov, \& Lu, 2009; Kuai, Zhang, Klein, Levi, \& Yu, 2005; R. Wang, Zhang, Klein, Levi, \& Yu, 2012; Xiao et al., 2008).

Different ideas have developed about the conditions under which perceptual learning is (or is not) specific for stimulus features and position. According to the lowest-level hypothesis (Karni \& Bertini, 1997), prolonged training will lead to plasticity at the lowest levels in the visual system where the trained stimulus is best represented. Plasticity at low levels of the visual system, where neurons have small receptive fields and are precisely stimulus-tuned is thought to lead to specificity of the trained visual skill. The reverse hierarchy hypothesis, predicts that easy tasks generalize, while more difficult ones will lead to specificity for the same amount of training (M. Ahissar \& Hochstein, 1997, 2004; Hochstein \& M. Ahissar, 2002). This is based on the concept that easier tasks depend on read-out from relatively higher levels of the visual hierarchy, while more difficult tasks requiring finer analysis depend on read-out from neurons in lower levels, where neurons show smaller receptive fields and show precise stimulus tuning. A related idea is that specificity 
depends on the precision of the task with which transfer is tested (transfer task) after initial training on another task (Jeter et al., 2009). Others, in contrast, showed that the difficulty of the training task (i.e., the sensory challenge), but not the difficulty of the transfer task, determined whether there was generalization (X. Wang, Zhou, \& Liu, 2013). Furthermore, it has been suggested that the number of trials determines the degree of specificity (Aberg et al., 2009; Censor \& Sagi, 2009; Jeter et al., 2010). This is in line with a recent report from Hung and Seitz (2014), who showed that prolonged training at threshold leads to retinotopic specificity. The importance of repeated presentations of the training stimuli is also in line with a contribution of adaptation to learning and specificity as proposed by Harris, Gliksberg and Sagi (2012). They showed that when adaptation was prevented, position specificity was abolished arguing for a contribution of sensory adaptation in low level areas to position specificity. Attentional mechanisms contribute strongly to perceptual learning (E. Ahissar et al., 1992; M. Ahissar \& Hochstein, 1993; Karni \& Sagi, 1991 , 1993), but it is not clear to what extent attention enhances retinotopic position specificity beyond what is induced by adaptation mechanisms.

Taken together, the reviewed literature indicates that perceptual skills can become specific to stimulus features and position after sufficiently long (asymptotic) learning in a task that pushes the sensory limits of the system. This concept has been challenged in orientation discrimination experiments in which training at a given position generalized to another position if that position was pre-exposed to the stimulus in the context of an irrelevant task (R. Wang et al., 2012; Xiao et al., 2008; J.-Y. Zhang et al., 2010). Moreover, the same group (T. Zhang, Xiao, Klein, Levi, \& $\mathrm{Yu}, 2010)$ also showed that a brief pre-test was sufficient to enable complete transfer from foveal to peripheral positions in an orientation discrimination task using Gabor stimuli and unidimensional noise fields as used by Schoups et al. (1995). Their claim of complete generalization of orientation discrimination across trained and exposed positions was interpreted as a challenge to the view that plasticity in retinotopic areas could contribute to position specificity, as had been supported by prior studies (Schoups et al., 1995; Schoups et al., 2001). Xiao et al. (2008) suggested that perceptual learning "involves higher nonretinotopic brain areas that enable location transfer" (p.1922), and would suggest a complete transfer of 
training-induced read-out enhancement across the visual field. However, an alternative interpretation would be that the complete generalization observed in Xiao et al. (2008) could be a phenomenon restricted to the early phases of learning to which Xiao et al.'s (2008) and Zhang et al.'s (2010) studies were restricted. This interpretation is in line with other observations of transfer, when transfer tasks are easy, or when training is short and does not include asymptotic learning, or when few trials at threshold are presented (e.g. E. Ahissar et al., 1992; Hung \& Seitz, 2014; Jeter et al., 2009).

Here, we aimed to test the specificity/generalization of Gabor orientation discrimination learning after an extended period of training. In addition, we wished to test whether specificity could be predicted from the extent of learning, to gain insight into mechanisms contributing to specificity. Thirdly, we arranged the training and generalization-test positions along a line of equal polar angle in an attempt to detect eccentricity effects upon generalization. We reasoned that if specificity (and generalization) were entirely due to processes in non-retinotopic high-level areas, expertise should spread equally in all directions around the trained location. Alternatively, if retinotopic areas, which are characterized by cortical magnification of central vision (Sereno et al., 1995), contribute to specificity then generalization in the visual field should be greater towards the periphery than towards the center of vision.

We present data from ten participants trained for three weeks on an orientation discrimination task in which stimuli were presented around a $135^{\circ}$ reference orientation, using Gabor stimuli at $6^{\circ}$ eccentricity, equidistant from horizontal and vertical meridians. After completion of training, participants were tested at different eccentricities $\left(3^{\circ}, 6^{\circ}, 9^{\circ}, 12^{\circ}\right.$, and $15^{\circ}$ along a line of equal polar angle), to test possible transfer of orientation discrimination performance. Importantly, we did not include a pre-test of transfer positions since it has been shown that even very few trials can lead to very substantial position generalization (T. Zhang et al., 2010). After completion of training, participants were tested in all five test positions at an orientation orthogonal to the trained reference orientation. In addition, naïve participants were tested at different eccentricities along a line of equal polar angle. These additional experiments were designed to test whether position specificity 
would be restricted to the trained reference orientation, and to verify whether any observed differences among the test positions at different eccentricities reflected truly a generalization of skill, or instead an interaction between eccentricity and characteristics of the Gabor stimulus such as its contrast and spatial frequency. In our analysis, we furthermore aimed to test whether the evidence for position and orientation specificity would be related to the magnitude of asymptotic learning. 


\section{Methods}

\section{Participants}

Ten participants (mean age 25.51, SD 5.62, 6 female) naïve to the purpose of the study participated in the main experiment and the control conditions with differing reference orientation. Additionally, eight naïve participants (mean age 21.38, SD 2.34, 7 female) were recruited for testing the effect of eccentricity and spatial frequency on orientation discrimination performance. All participants had normal or corrected-to normal visual acuity. Informed, written and verbal consent was obtained according to the Helsinki Declaration, after full information about all procedures and the right to withdraw participation at any time. All procedures were approved by the local Ethical Committee of the Faculty of Psychology and Neuroscience (ECP). For their participation in the study participants received monetary reward, credits to fulfill course requirements, or a combination of both.

\section{Task, Stimuli, and Apparatus}

Participants performed an orientation discrimination task with an oblique orientation as an implicit reference stimulus. They indicated the deviation of a Gabor stimulus from the reference orientation by pressing either the left or the right arrow key for counterclockwise and clockwise deviations respectively (Figure 1A). In all learning experiments, Gabor patches around the $135^{\circ}$ reference, or the $45^{\circ}$ reference in the control condition were used as stimuli (2.37 cycles/degree spatial frequency, 50\% Michelson contrast, $0.5^{\circ}$ standard deviation, 4 different phases: $0^{\circ}, 45^{\circ}, 90^{\circ}$, and $135^{\circ}, 3^{\circ}$ diameter, $500 \mathrm{~ms}$ duration). The Gabor stimulus was presented at eccentricities of $3^{\circ}, 6^{\circ}, 9^{\circ}, 12^{\circ}$, and $15^{\circ}$, and its average luminance was $56 \mathrm{~cd} / \mathrm{m}^{2}$, which was equal to the luminance of the background. The most foveal $\left(3^{\circ}\right)$ and peripheral positions $\left(15^{\circ}\right)$ were chosen such that they were approximately equidistant in V1 in terms of cortical distance (Sereno et al., 1995) from the trained position. The stimuli were presented in the upper left or right hemifield (counterbalanced over participants). In one control experiment (see end of next section), we used a Gabor at higher spatial frequency ( 8.04 cycles/degree). In all experiments, the fixation dot was a small, white disc $\left(0.2^{\circ}\right)$. Participants were placed in a dimly lit room; their head was supported by a chin and head rest keeping eye-screen distance 
constant at $57 \mathrm{~cm}$. Visual stimuli were displayed on a 19" Samsung SyncMaster 940BF LCD monitor (Samsung, Seoul, South Korea; 60Hz refresh rate, 1280x1024 resolution). The screen was covered by a gray mask with an oval aperture so that the screen borders were not visible to participants and thus could not be used as reference for the orientation discrimination task (the center of the Gabor stimuli was always at least $18.5^{\circ}$ from the exterior border of the mask). Fixation control was monitored with a Viewpoint Eyetracker v.2.8.3 (Arrington Research, Inc., Scottsdale, Arizona, USA; $60 \mathrm{~Hz}$ sampling rate). Stimulus presentation and response recording was performed by Cortex v.5.9.6 (NIH freeware for psychophysical and neurophysiological experimentation). Since the screen dimensions did not permit the presentation of a stimulus at $16^{\circ}$ eccentricity while having the fixation spot in the middle of the screen, the screen in that condition was shifted $7 \mathrm{~cm}$ up and $7 \mathrm{~cm}$ to the left or right (depending on the tested visual field quadrant). The fixation spot was moved oppositely, keeping it in the center of vision. Both the main and control experiment were done with a gaze-contingent display in which trials only started after a period of accurate fixation within a $3^{\circ}$ by $3^{\circ}$ window centered on fixation. Trials were aborted if the participant's gaze fell outside that window.

\section{Training protocol}

To compare performance on trained and untrained positions, participants first underwent an extensive training phase resulting in asymptotic performance levels. Over the course of 10-15 consecutive working days participants performed the orientation discrimination task (Figure 1A) on a $135^{\circ}$ oblique stimulus in an upper quadrant at $6^{\circ}$ eccentricity (Figure 1B). The side of the training quadrant was counterbalanced across participants. A training session consisted of 1 or 2 blocks of 4 staircase measurements determining $84 \%$ correct orientation difference thresholds (see Data and Statistical analyses). The complete training program led to learning curves composed of at least 60 staircases. Seven participants were trained for fifteen sessions of four staircases per session (60 thresholds), whereas three more participants (P2-4) were trained for 10 sessions with 8 staircases per session (total of 80 thresholds). In three participants (P8-10) with 15 sessions, training was supplemented with 26-39 refresher thresholds (see Results section). Refresher thresholds were aimed to counter potential forgetting in participants who were 
invited to perform the generalization testing several months after completion of training. The time delay between end of learning and refresher thresholds was 4,10 , and 10 months in P8, P9and P10, respectively. Generalization testing immediately followed the refresher threshold measurements in P8-10, or the end of learning in the other participants. Note that the determination of position specificity relied only on a post-training comparison of thresholds obtained in the trained position with thresholds in surrounding test positions. We did not perform a pre- training versus

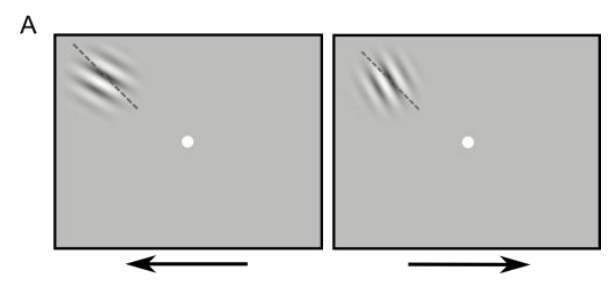

B

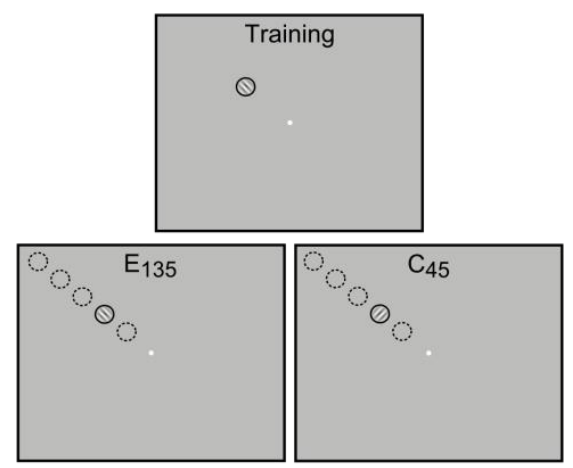

Figure 1. Orientation discrimination task and experimental design. (A), Stimuli and task. Thresholds were determined using an identification design, with left key responses for counterclockwise orientation offsets from a $135^{\circ}$ reference (dashed line, not shown to participants), and right key responses for clockwise orientation offsets. Here, stimuli are enlarged for illustration. (B), Training phase (top) and the two experimental conditions of the testing phase (bottom), one being the experimental condition $\left(\mathrm{E}_{135}\right)$ and the other the control condition $\left(\mathrm{C}_{45}\right)$. Solid circles represent the position $\left(6^{\circ}\right)$ at which training took place. Dashed circles indicate the positions at which generalization was tested: $3^{\circ}, 9^{\circ}, 12^{\circ}$, and $15^{\circ}$. Dashed and solid circles were not present in actual display and are shown here only for the purpose of illustration. In the $\mathrm{E}_{135}$ and $\mathrm{C}_{45}$ conditions, a Gabor stimulus is shown for illustration in the position trained at the $135^{\circ}$ reference orientation. Here, the Gabor stimuli are shown to scale. 
post-training comparison in the test locations surrounding the trained position. This would have required including some pre-learning threshold measurements in these test locations. We purposefully opted against this option to avoid the possibility that transfer could be due to limited pre-training measurements in the different test locations (see T. Zhang et al., 2010). Here, we are interested in the amount of transfer to other locations (or the lack thereof referred to as specificity) induced by training at a single location, not contaminated by any prior experience in those locations.

The transfer phase of the experiment consisted of two different conditions (lower part of Figure $1 \mathrm{~B})$; one experimental $\left(\mathrm{E}_{135}\right)$ and one control condition $\left(\mathrm{C}_{45}\right)$. In both of these conditions, five different eccentric positions were tested: $3^{\circ}, 6^{\circ}$ (trained position), $9^{\circ}, 12^{\circ}$, and $15^{\circ}$. In the $\mathrm{E}_{135}$ condition, eight staircases at the $135^{\circ}$ reference were completed per position over two subsequent days, resulting in 40 threshold measurements. In the $\mathrm{C}_{45}$ condition, another 8 staircases per position were collected at the $45^{\circ}$ reference, yielding another 40 thresholds. On each day, positions were presented in a pseudorandom manner. The experimental condition $\left(\mathrm{E}_{135}\right)$ was carried out first, followed by the control condition $\left(\mathrm{C}_{45}\right)$.

In a control experiment we tested the effect of spatial frequency and eccentricity on orientation discrimination performance in a group of naïve participants $(n=8)$. Two Gabor patches with different spatial frequency were used. The low spatial frequency stimulus was identical to the stimulus used in the main experiment (i.e., 2.37 cycles/degree), the high spatial frequency stimulus had a spatial frequency of 8.04 cycles/degree. Participants completed two sessions, in each of which the orientation discrimination task was performed at four different eccentric positions $\left(3^{\circ}, 6^{\circ}, 9^{\circ}\right.$, and $\left.12^{\circ}\right)$ with one of the two spatial frequencies. In total, 4 thresholds were obtained at each position with the standard Gabor and with the higher frequency Gabor. The 4 positions were arranged along an equi-polar line of $45^{\circ}$ (upper right quadrant, stimulus orientation $135^{\circ}, 4$ participants) or $135^{\circ}$ (upper left quadrant, stimulus orientation $45^{\circ}, 4$ participants). The order of sessions was counterbalanced across participants as was the order of stimulus positions. 


\section{Data collection and statistical analyses}

Thresholds were measured using a Wetherill \& Levitt (1965) staircase tracking 84\% correct performance, in which 4 correct responses in a row led to a decrease in the orientation difference (division by 1.2) and a single incorrect response to an increase (multiplication by 1.2). The staircase was terminated either when 14 reversal points were acquired or 120 trials were completed whichever came first. For the first training session, the start level of the four staircases was set at an orientation difference of $\pm 15^{\circ}$. This high starting level in the first session was chosen because it is likely above threshold for most participants, but not so high that the thresholdlevel could not be reached within a single staircase. With this starting position, a level close to the typical first-session threshold (mean $11.44^{\circ}, \mathrm{SD} 6.75, \mathrm{n}=10$ ) can be reached in about two steps, or eight correct responses. These initially clear orientation differences can be considered as a way to facilitate subsequent learning (Eureka effect, see M. Ahissar \& Hochstein, 2004). For all subsequent sessions, including experimental and control conditions, the average threshold level of the previous session was taken as a starting point. This was done to maximize the number of trials given around threshold level. Despite the different starting rule between the first session and the later ones, staircases in the first session showed similar statistics. The last 10 reversal points used for threshold calculation corresponded to an $84.9 \%$ performance level in the first session, within the $82.5-$ $85.9 \%$ range for all additional sessions (averaged over all participants). The average length of the staircases of the first session (93 trials) was only slightly longer than that for the other sessions (85-95 trial range). These observations indicate that all thresholds shown during learning from first to last session reflect a good estimation of the same $84 \%$ correct performance level, and hence that the first-session threshold and the faster rate of learning in the beginning part of the learning was not due to a different functioning of the staircases during initial learning. The staircase threshold was calculated as the geometric mean of the last ten reversal points; the first four reversal points were excluded. Statistical analysis was based on average thresholds in each condition per participant, with each participant contributing one data point per condition. Of each averaged participant's threshold in a condition, the natural logarithm was taken to homogenize variance. 
Data from the experimental condition $\mathrm{E}_{135}$ of all ten participants were analyzed with a repeated-measures ANOVA to investigate the extent to which training in one position transferred to different neighboring positions. Furthermore, to test for position specific effects of training, and ensure that these effects were orientationspecific and not due to a non-specific interaction between stimulus properties and eccentricity, the experimental condition $\mathrm{E}_{135}$ was compared to the control condition $\mathrm{C}_{45}$. To assess the influence of learning itself on orientation discrimination performance at different positions, a mixed-design ANOVA with position as withinsubject factor and training as between-subject factor was done, using data from the control experiment. In cases where the sphericity assumption was violated, Greenhouse-Geisser correction was applied. For pairwise comparisons, two-sided ttests were performed and adjusted for multiple comparisons using Bonferroni correction.

To quantify position specificity and training-related performance increases, we calculated indices based on the Michelson index. The training index quantified the amount of performance increase for the total length of training, or within a specific phase of training and was calculated as:

$$
T=\frac{T r_{\text {begin }\left(6^{\circ} e c c\right)}-T r_{\text {end }\left(6^{\circ} e c c\right)}}{\operatorname{Tr} \text { begin }\left(6^{\circ} e c c\right)}+\operatorname{Tr} r_{\text {end }\left(6^{\circ} e c c\right)}
$$

$\operatorname{Tr}_{\text {begin( }\left(6^{\circ} \text { ecc) }\right.}$ and $\operatorname{Tr}_{\mathrm{end}\left(6^{\circ} \text { ecc) }\right.}$ represent the average of the first and last group of 4 staircase thresholds obtained in a specific training phase at the eccentricity position of $6^{\circ}$. The mean learning curve averaged over all participants was based on 60 individual thresholds. Hence, thresholds used to compute the training index for the whole learning curve compared the average of individual thresholds 1-4 with the average of individual thresholds 57-60. Training indices were also calculated separately for the first, middle, and last third of the learning curve. Some participants had done more than 60 staircase thresholds because of a different training protocol (P2-4) or additional refresher thresholds (P8-10). Therefore, for these participants, we also calculated the full and the late training index $\mathrm{T}$ using end thresholds 77-80 in P2-4 and the last 4 refresher thresholds in P8-10 (see Results). 
The position specificity index quantified the difference between performance at the trained position and performance at all other test positions and was calculated as:

$$
P S=\frac{T e_{\left(6^{\circ} e c c\right)}-\overline{T e}_{(\text {other ecc })}}{T e_{\left(6^{\circ} e c c\right)}+\overline{T e}_{(\text {other ecc })}}
$$

Here, $T e_{\left(6^{\circ} \text { ecc }\right)}$ represents the average threshold from the trained position in condition $\mathrm{E}_{135}$ during the transfer phase, and $\overline{T e}_{(\text {other ecc) }}$ average threshold from all other (pooled) positions in the same condition.

The orientation specificity index quantified the difference between performance at the trained position for the trained orientation $\left(135^{\circ}\right)$ and the control orientation $\left(45^{\circ}\right)$ and was calculated as:

$$
O S=\frac{T e_{\left(45^{\circ}\right)}-T e_{\left(135^{\circ}\right)}}{T e_{\left(45^{\circ}\right)}+T e_{\left(135^{\circ}\right)}}
$$

Here, $T e_{\left(45^{\circ}\right)}$ represents the average threshold from the untrained orientation in condition $\mathrm{C}_{45}$ at the trained position during the transfer phase, and $T e_{\left(135^{\circ}\right)}$ represents the average threshold from the trained orientation in condition $\mathrm{E}_{135}$ at the trained position. For all indices, we performed 1.5 Interquartile Range (IQR) criterion outlier detection, to exclude biasing any correlations based on those indices by extreme data points. We used ordinary least square regression analysis to predict position and orientation specificity from learning indices. Robust regression analysis was additionally used to further test for the contribution of extreme data points.

Additionally, a generalization asymmetry index was calculated to quantify the difference in generalization from the trained $6^{\circ}$ position to the $3^{\circ}$ and $9^{\circ}$ testing positions respectively. It was calculated as the difference in threshold at $3^{\circ}$ and $9^{\circ}$ test positions. 


\section{Results}

\section{Learning curves}

Figure 2 shows data from individual participants in 10 separate panels, showing training at the $135^{\circ}$ reference orientation on the left and the transfer test (position and orientation specificity test) on the right. On the left in each panel, the complete training phase is shown for 15 sessions of four thresholds in seven participants, or for 10 sessions of 8 thresholds in three other participants (P2-4). In three participants (P8-10), refresher thresholds are shown following the learning curve. These thresholds were collected just prior to generalization testing to counter possible forgetting due to a time delay in inviting these participants for the generalization test (see Methods). Overall, participants showed considerable learning during the training phase at the $135^{\circ}$ oblique reference orientation. On the right in each panel, performance in the transfer-testing phase is shown by comparing the average threshold at the previously trained position with the average pooled threshold from all other generalization-test positions (as tested with eight thresholds per participant in each test position). This comparison was done separately for the $\mathrm{E}_{135}$ and $\mathrm{C}_{45}$ generalization tests. In the individual data in Figure 2, averages corresponding to the first and second block of 4 staircases in each condition are shown separately. Overall, the transfer data in the $\mathrm{E}_{135}$ condition suggest a trend for test thresholds at the $135^{\circ}$ reference orientation to be higher in non-trained (dashed dark grey line) than in the trained position (solid dark grey line). This difference is not systematically present for test thresholds measured in the $\mathrm{C}_{45}$ condition $\left(45^{\circ}\right.$ reference orientation, light grey solid and dashed lines). Note that overall, thresholds in the $\mathrm{C}_{45}$ condition exceeded those in the $\mathrm{E}_{135}$ condition.

Figure 3 shows the data averaged over all ten participants. Given the slightly different ways in which training data were collected in P2-4, and the presence of refresher thresholds in P8-10, we took the first 60 thresholds of all ten participants and divided them into blocks of four thresholds, resulting in 15 data points for the learning curve in each participant. This means that neither the last 20 thresholds of P2-4, nor the refresher thresholds in P8-10 were included. Hence each data point in 

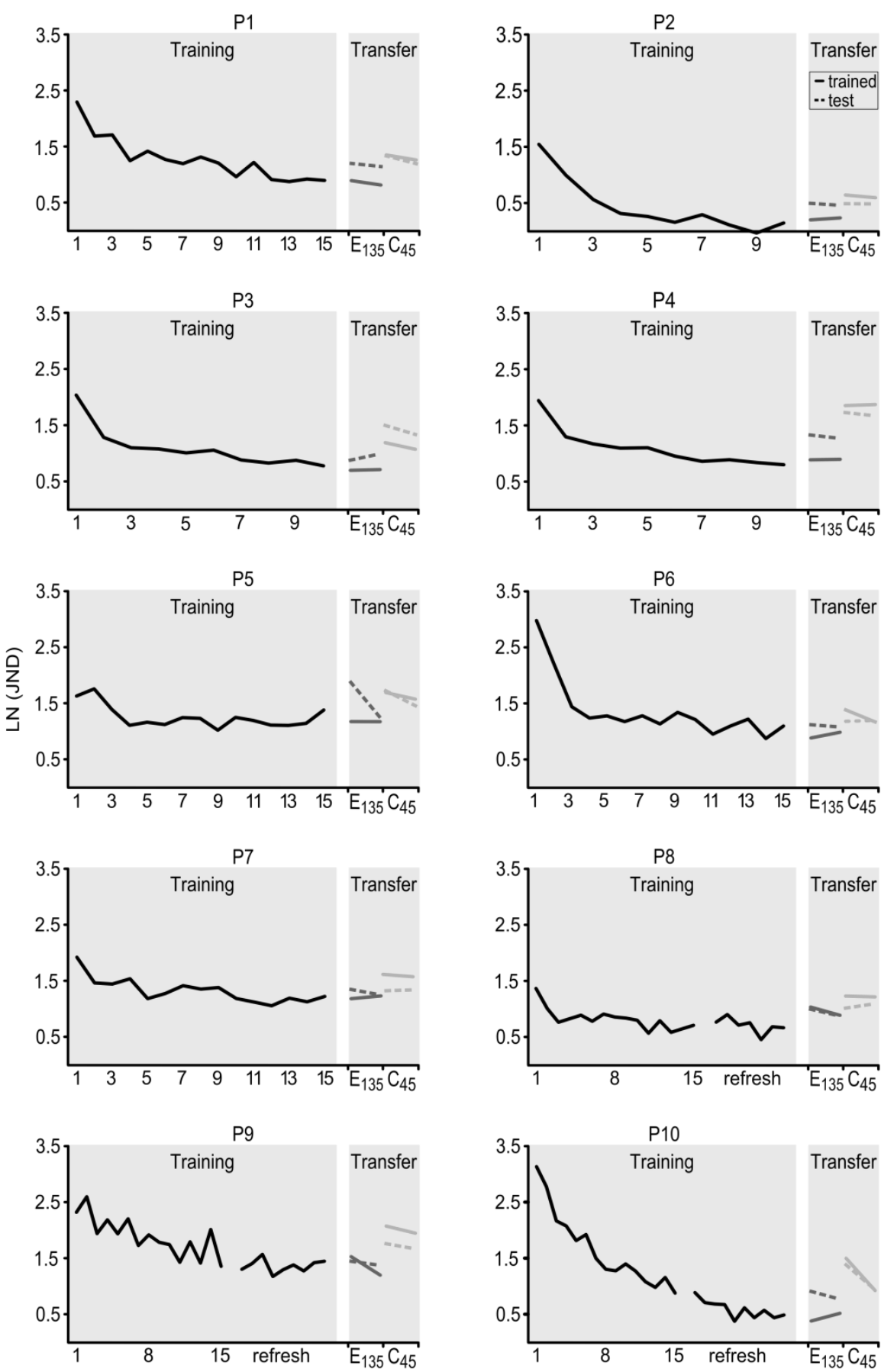

Session 
Figure 2. Individual learning curves for all ten participants. Participants completed slightly different training schemes: In participants 1-7, training was followed by tests of position specificity. Participants 8-10 received their training $\sim 6$ months prior to position generalization testing, and therefore refresher thresholds(26-39 thresholds) were given prior to generalization testing. In all participants, the quadrant where training occurred had never been exposed to any preceding training with visual stimuli. Of the ten participants, three had no experience in prior orientation discrimination tasks and were trained only for the purpose of the present experiment in at the $135^{\circ}$ reference orientation at the $6^{\circ}$ position along the $135^{\circ}$ polar angle line prior to position generalization testing (participants 2-4). The other seven had experience at other reference orientations in quadrants other than the one used for the present study (which in further analysis we showed not to contribute to the reported patterns of results here reported). In the generalization test phase (TesT), $\mathrm{E}_{135}$ (dark grey lines) refers to the main experimental condition in which participants performed the orientation task on a $135^{\circ}$ reference orientation. $\mathrm{C}_{45}$ (light grey lines) is the control condition where the same task is performed on a $45^{\circ}$ reference orientation. Solid lines refer to thresholds at the trained position, whereas dashed lines refer to the mean threshold at all other test positions.

the learning curve corresponds to 10 average thresholds, one per participant, with each data point in an individual representing the average of 4 individual staircase measurements. In the transfer phase, a difference was present in the $\mathrm{E}_{135}$ condition between thresholds in the standard trained position (dark grey square symbol) and in the test positions (dark square). An analogous difference was not present for the $\mathrm{C}_{45}$ condition (light grey symbols). Here, each data point represents the average of 10 participants, each contributing 8 individual staircase measurements.

The question can be asked a priori whether including the extra data would yield different conclusions for our analysis of position specificity. In our analyses, we found that conclusions did not depend on including or excluding the extra data (see Position specificity and training indices and Orientation specificity and training indices for details). This is not surprising as the extra data never showed a threshold increase over time, and only in 2 out of 6 participants (P2-4 and P8-10) was there a significant threshold decrease. In participants P2-P4, we compared the last 20 thresholds (excluded in Figure 3) with the previous 20thresholds (included) and there was no significant difference $\left(\mathrm{P} 2: t_{(19)}=1.675, p=.110 ; \mathrm{P} 3: t_{(19)}=1.065, p=\right.$ $\left..300 ; \mathrm{P} 4: t_{(19)}=1.151, p=.264\right)$. In participants P8-10, we compared the last 20 
thresholds of training (thresholds 40-60) with the last 20 refresher thresholds and there was a significant decrease in participants P9 and P10 (P9: $t_{(19)}=3.095, p=$ .006 ; P10: $\left.t_{(19)}=9.405, p<.001\right)$ but not participant P8 $\left(t_{(19)}=-0.146, p=.886\right)$.

To statistically test the data obtained during generalization testing, we used a repeated measures ANOVA on the data as shown in the Transfer panel of Figure 3. This analysis revealed a significant main effect of condition (experimental, control) $\left(F_{(1,9)}=36.927 ; p<.001\right)$, a non-significant main effect of test position (trained, others) $\left(F_{(1,9)}=3.356 ; p=.100\right)$, and an interaction between these two factors $\left(F_{(1,9)}\right.$ $=25.971 ; p=.001)$. As the interaction indicated that the position effect differed for the experimental and control conditions, a repeated measures ANOVA on position (trained, others) was performed for condition $\mathrm{E}_{135}$ and $\mathrm{C}_{45}$ separately. For the $\mathrm{E}_{135}$ condition there was a highly significant effect of position $\left(F_{(1,9)}=22.628 ; p=.001\right)$, with the orientation discrimination test thresholds at the trained position

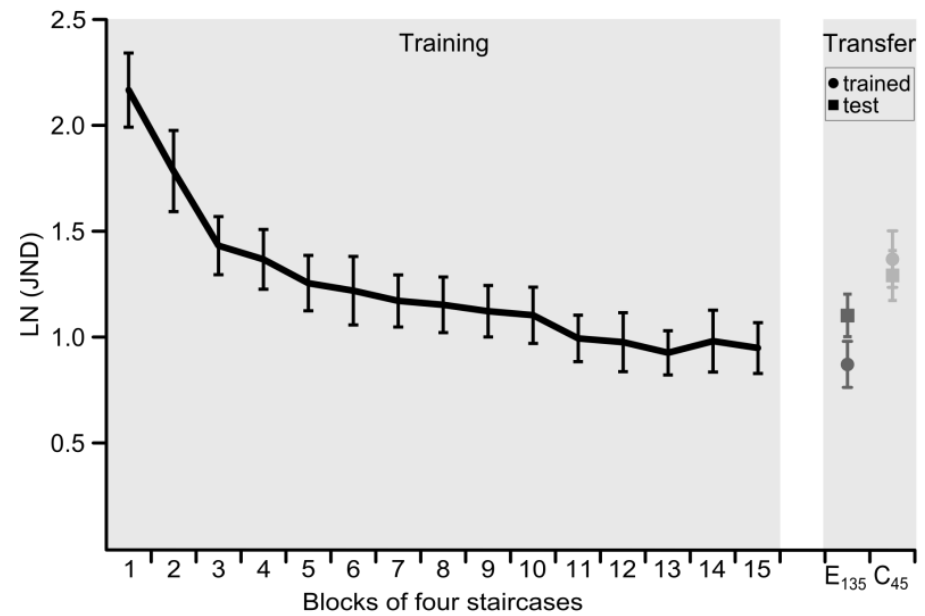

Figure 3. Mean performance during training and testing phase. Each data point of the learning curve (black) corresponds to the average of 10 thresholds ( 1 per participant), with each participant's threshold based on 4 staircases. In three participants, blocks do not correspond to daily sessions (details in text). The data from the transfer phase (one data point per participant, based on the average of 8 staircase thresholds) shows thresholds for $135^{\circ}$ (dark gray symbols) and $45^{\circ}$ (light gray symbols) stimuli conditions at the trained position (round symbols) and the average of all generalization test positions (square symbols). Error bars represent the standard error of the mean. 
(Figure 3, dark grey dot) being lower than the average of all other test positions (Figure 3, dark square symbol). In the $\mathrm{C}_{45}$ condition (light grey symbols), in which orientation discrimination was tested with the untrained $45^{\circ}$ reference orientation, the repeated measure ANOVA resulted in no significant main effect of position $\left(F_{(1,}\right.$ 9) $=1.821 ; p=.210)$. This indicates that the emergence of position specificity at the end of learning was specific for the trained reference orientation. Interestingly, overall, the thresholds in the $\mathrm{C}_{45}$ conditions in Figure 3 were considerably worse than the learning asymptote of the trained $135^{\circ}$ reference orientation, returning roughly to the performance level observed in the fourth training session. This suggests a relatively large amount of orientation specificity. At the trained $6^{\circ}$ location, the difference between thresholds at the $45^{\circ}$ and $135^{\circ}$ reference orientations was relatively large (compare dots), and significant $\left(t_{(9)}=-6.823, p<\right.$ $.001)$. The difference between thresholds at the $135^{\circ}$ reference orientation in the previously trained versus untrained positions was slightly smaller (compare dark grey symbols), but still significant $\left(t_{(9)}=-4.757, p=.001\right)$. Hence, the data revealed a larger orientation specificity than position specificity $\left(t_{(9)}=-4.267, p=.002\right)$.

The evidence for orientation specificity can be interpreted further within the generalization data. In the test positions that were neither trained at the $45^{\circ}$ nor the $135^{\circ}$ reference orientations (compare square symbols), there was nevertheless some evidence for orientation specificity ( $\mathrm{E}_{135}$ test vs. $\mathrm{C}_{45}$ test: $t_{(9)}=-3.527, p=.006$ ). This indicates that there can be orientation specificity in locations that have not been exposed to any training, but at threshold levels that exceed the level attained after complete training. For the untrained orientation $\left(45^{\circ}\right)$ the difference in thresholds between trained and untrained positions was not significant (compare light grey symbols: $t_{(9)}=1.349, p=.210$ ), demonstrating that position specificity did not extend to the untrained orientation. Accordingly, the difference in threshold between trained and non-trained locations for the $135^{\circ}$ reference orientation was significantly larger than the difference in thresholds between trained and non-trained locations for the $45^{\circ}$ reference orientation $\left(t_{(9)}=5.096, p=.001\right)$. This further supports the idea that full training at a $135^{\circ}$ reference orientation at the $6^{\circ}$ eccentricity position creates a form of expertise that is linked to the trained position, and not just to the trained reference orientation. 
Overall, the data also show that the variation in thresholds due to position and orientation specificity was small compared to the decrease in thresholds from the beginning to the end of learning. Moreover, the data show that orientation specificity appeared a more robust effect than position specificity. Hence, the data indicate that much, but not all, of the performance benefit of learning can generalize over position and orientation (see Position specificity and training indices and Orientation specificity and training indices).

\section{Stimulus position effect in terms of eccentricity}

To test the position specificity of orientation discrimination training in a more detailed manner, we performed a repeated measures ANOVA with thresholds of each of the five eccentric positions $\left(3^{\circ}, 6^{\circ}, 9^{\circ}, 12^{\circ}\right.$ and $\left.15^{\circ}\right)$ as a within-subjects factor. The results showed that the main effect of stimulus position was highly significant $\left(F_{(4,36)}=10.869 ; p<.001\right)$. Pairwise comparisons showed that thresholds at the most central position differed from thresholds at all other eccentricities (Figure 4A) except for the most peripheral one $\left(3^{\circ}\right.$ vs. $6^{\circ}-t_{(9)}=5.785, p=.003 ; 3^{\circ}$ vs. $9^{\circ}-t_{(9)}=4.920, p=.008 ; 3^{\circ}$ vs. $12^{\circ}-t_{(9)}=4.260, p=.021 ; 3^{\circ}$ vs. $16^{\circ}-t_{(9)}=$ 3.131, $p=0.121)$. The remaining comparisons between positions did not differ significantly from each other $\left(6^{\circ}\right.$ vs. $9^{\circ}: t_{(9)}=-2.268, p=.495 ; 6^{\circ}$ vs. $12^{\circ}: t_{(9)}=-$ $3.317, p=.120 ; 6^{\circ}$ vs. $16^{\circ}: t_{(9)}=-3.500, p=.067 ; 9^{\circ}$ vs. $12^{\circ}: t_{(9)}=0.175, p>.999$; $9^{\circ}$ vs. $16^{\circ}: t_{(9)}=0.172, p>.999 ; 12^{\circ}$ vs. $\left.16^{\circ}: t_{(9)}=0.015, p>.999\right)$. Hence, the data seem to suggest that the expertise gained at the $6^{\circ}$ trained position generalized more towards peripheral positions than to the more central position. This asymmetric effect of distance when comparing the trained position with central vs. peripheral visual field positions may suggest the involvement of low-level visual areas with cortical magnification of central vision (Sereno et al., 1995). In this view, the relatively larger threshold increase at the most central $3^{\circ}$ position compared with the threshold at the $9^{\circ}$ position may be in agreement with the fact that the cortical distance (in retinotopic areas) corresponding to the $6^{\circ}$ to $3^{\circ}$ distance in the visual field is magnified compared to the cortical distance corresponding to the $6^{\circ}$ to $9^{\circ}$ distance in the visual field. Figure 4A suggests that after training, there is a performance advantage for eccentricities up to almost $15^{\circ}$. 
To evaluate whether the trend towards better performance in the peripheral test positions is a learning-related effect, or perhaps the consequence of an advantage of low spatial frequency stimuli at higher eccentricities (Kehrer, 1989, 1997; Kehrer \& Meinecke, 2006), we conducted a control experiment in eight naive participants without prior orientation discrimination training. They performed orientation discrimination with the same Gabor stimulus (2.37 cycles/degree) along eccentricities ranging from $3^{\circ}$ to $12^{\circ}$ along an equi-polar line in a single visual field quadrant (see Methods for details). In addition, we included testing at a higher spatial frequency (8.04 cycles/degree) to test interactions between spatial frequency and eccentricity. We did not include the largest eccentricity of $15^{\circ}$ in the experiment, because of the anticipated difficulty for naïve observers to perform at this eccentricity while maintaining fixation, and as most of the eccentricity effect in Figure $4 \mathrm{~A}$ was present in the $3^{\circ}$ to $12^{\circ}$ range.

Moreover, we wished to limit the experiment to two sessions, to avoid major effects of training. The data with the standard Gabor stimulus in the untrained participants no longer showed a trend towards decreased thresholds for higher eccentricities (Figure 4B, black line). In the repeated measures ANOVA, we

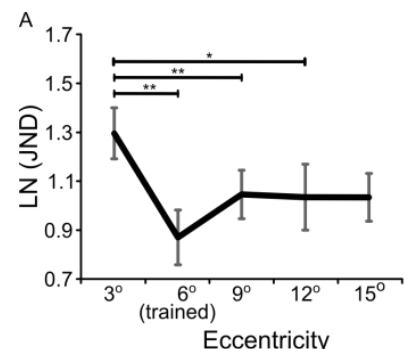

Eccentricity

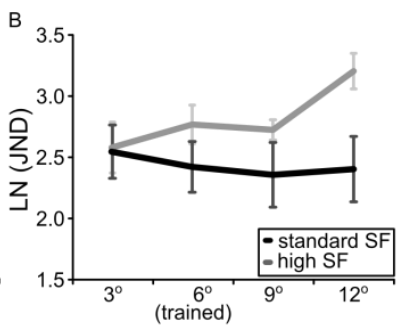

Eccentricity

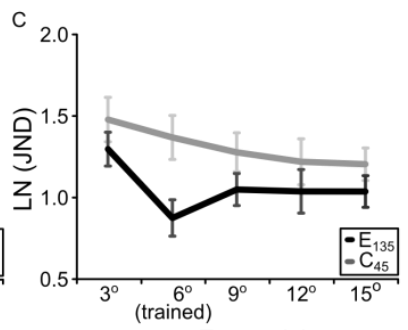

Eccentricity

Figure 4. Orientation discrimination thresholds as a function of stimulus position eccentricity. $A$, Participants that completed 15 sessions of training at the reference orientation of $135^{\circ}$ performed the transfer test at reference orientations of $135^{\circ}$ (black line: $\left.\mathrm{E}_{135}\right) .(* p<.05$, ** $p$ $<.001)$. B, Naïve control participants without any prior training performed orientation discrimination at either a $135^{\circ}$ or a $45^{\circ}$ reference orientation (depending on the upper quadrant chosen for stimulus presentation) on four different stimulus position eccentricities (black: standard spatial frequency, grey: high spatial frequency). $\boldsymbol{C}$, Participants that completed 15 sessions of training at the reference orientation of $135^{\circ}$ performed the transfer test also at reference orientations of $45^{\circ}$ (grey line: $\mathrm{C}_{45}$ ). Error bars show standard error. 
included data from both the low and high spatial frequency condition and eccentricity $\left(3^{\circ}, 6^{\circ}, 9^{\circ}, 12^{\circ}\right)$. We found a significant main effect of spatial frequency $\left(F_{(1,7)}=7.694, p=.028\right)$, a non-significant main effect of eccentricity $\left(F_{(3,21)}=\right.$ 2.564, $p=.082)$ and an almost significant interaction $\left(F_{(3,21)}=2.908, p=.059\right)$, which was due to a small trend for increasing thresholds as a function of eccentricity with the high frequency Gabor (see grey line in Figure 4B). Additional analysis confirmed that orientation discrimination thresholds for the standard Gabor stimulus showed no performance increase with increasing eccentricity $\left(F_{(3,21)}=0.527, p=\right.$ $.669)$.

In the experimental design, we also included session order or position order, but these factors did not affect performance (session order effect: $F_{(1,7)}=2.001, p=$ .200 ; position order effect $F_{(3,21)}=0.726, p=.548$; interaction: $F_{(3,21)}=1.445, p=$ .258). The lack of an effect of eccentricity on discrimination with the standard lowfrequency Gabor in the analyses, and the fact that eccentricity did not interact very strongly with the sizeable spatial frequency manipulation suggest that the eccentricity effect observed in our training experiment may to some extent reflect a learning effect, rather than an interaction between stimulus characteristics and eccentricity.

Participants also performed the orientation discrimination task at an untrained $45^{\circ}$ reference orientation in all five test positions. In the previously trained position (at $6^{\circ}$ ) this gave us the opportunity to test for generalization of training across orientations. In the non-trained test positions, we wished to test whether the trend for better performance at higher eccentricities would generalize to the untrained $45^{\circ}$ reference orientation. Considering the data for the $45^{\circ}$ reference orientation alone (dark grey line in Figure 4C), orientation discrimination improved with eccentricity (repeated measures ANOVA, $F_{(4,36)}=5.667, p=.001$ ). We also performed a repeated measures ANOVA combining the data from the two reference orientations. The analysis included reference orientation $\left(\mathrm{E}_{135}, \mathrm{C}_{45}\right)$ and position $\left(3^{\circ}, 6^{\circ}, 9^{\circ}, 12^{\circ}\right.$ and $15^{\circ}$ ) as within-subjects factors. It revealed a significant main effect of reference orientation $\left(F_{(1,9)}=22.981 ; p=.001\right)$, a significant main effect of eccentric position $\left(F_{(4,36)}=9.078 ; p<.001\right)$ and a significant interaction $\left(F_{(4,36)}=6.356 ; p=.001\right) . \mathrm{A}$ comparison of orientation discrimination thresholds for reference orientations at $45^{\circ}$ 
and $135^{\circ}$ per eccentricity revealed that all thresholds except at $15^{\circ}$ were significantly different $\left(3^{\circ}: p=.026 ; 6^{\circ}: p<.00008 ; 9^{\circ}: p=.009 ; 12^{\circ}: p=0.030 ; 15^{\circ}: p=.059\right)$. However, the interaction was mainly due to the difference between thresholds at the $6^{\circ}$ position, which indicates strong orientation specificity at that position. When excluding that position, a repeated measures ANOVA using reference orientation $\left(\mathrm{E}_{135}, \mathrm{C}_{45}\right)$ and position $\left(3^{\circ}, 9^{\circ}, 12^{\circ}\right.$ and $\left.15^{\circ}\right)$ as within-subjects factors confirmed the significant main effects of reference orientation $\left(F_{(1,9)}=12.721 ; p=.006\right)$ and eccentricity $\left(F_{(3,27)}=10.958 ; p<.001\right)$, but did not show a significant interaction $\left(F_{(3,27)}=0.232 ; p=.873\right)$.

Taken together, the data suggest that with training, participants acquired a skill specific for orientation at the trained $6^{\circ}$ position. In addition, there was a tendency for an asymmetric spread of orientation discrimination skill towards more eccentric positions. This tendency however was present for both the trained reference orientation and the orthogonal (non-trained) orientation. Remarkably, in all nontrained test positions except at the $15^{\circ}$ eccentricity, there was a significant trend for better performance at the $135^{\circ}$ reference orientation (trained at the $6^{\circ}$ position) than at the $45^{\circ}$ reference orientation. Except for the orientation specificity at the trained $6^{\circ}$ position, the effects were very small and we interpret them with caution. Overall, the asymmetric generalization of skill towards more peripheral visual field positions could be seen as in line with an involvement of lower level visual areas characterized by cortical magnification. The fact that this trend was present for both the trained and untrained reference orientation suggests that part of the peripheral advantage was due to an enhanced skill in attending to peripheral stimuli (or enhanced readout). The small advantage of the trained compared to the untrained reference orientation in test positions next to the trained position suggests an additional mechanism of spread of expertise in low-level areas independent of readout (unless read-out from unstimulated regions itself can become selective). 


\section{Position specificity and training indices}

The position specificity effects reported in Figures 2-4 were small compared to total learning. For more insight into the relative size of the training and position specificity effect, we calculated and compared training and position specificity indices. A paired sample t-test confirmed that the training index $(\mathrm{T}=0.40)$ was larger than the position specificity index (PS=0.15) $\left(t_{(9)}=-5.221, p=0.001\right)$. Hence, the variation in threshold performance among trained and untrained positions (position specificity index) was roughly three times smaller $(\mathrm{T} / \mathrm{PS}=2.85)$ than the variation in threshold performance between the beginning and end of learning (training index). This analysis was limited to progress made in the first 60 thresholds (as shown in Figure 3). When including all available data (extra thresholds 61-80 in P2-4 and refresher thresholds in P8-10), the ratio T/PS was 2.98.

Using the data from the first 60 thresholds in all participants, we then tested whether the training index was related to the position specificity index, and found a positive correlation $(\mathrm{r}=.77, \mathrm{R} 2=0.60, \mathrm{p}=.009)$. This indicates that the more participants improved during the training phase, the greater the position specificity effect was (Figure 5A). The training index significantly predicted position specificity
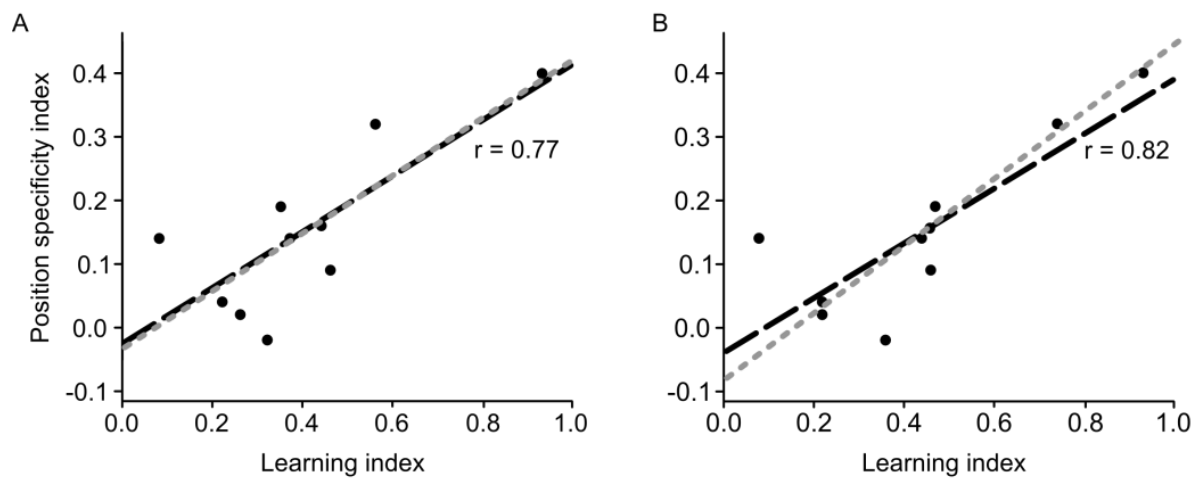

Figure 5. Scatterplot of position specificity and learning indices. $\boldsymbol{A}$, Position specificity correlates positively with the learning index. $\boldsymbol{B}$, The correlation between position specificity and learning index was even stronger when using the complete learning data (including extra thresholds and refresher training) for calculation of the training index. The black dashed line results from an ordinary least square regression, whereas the grey dotted line was fitted with betas obtained by using robust regression. 
$\left(\beta=0.44, t_{(9)}=3.438, p=.009\right)$ and also explained a significant proportion of variance in position specificity $\left(\mathrm{R}^{2}=0.60, F_{(1,9)}=11.800, p=.009\right)$. Robust regression yielded a slightly higher beta weight $\left(\beta=0.46, t_{(9)}=3.198, p=.013\right)$ indicating that outliers did not strongly drive the OLS regression results. This pattern of results was confirmed when including all available training data in all participants (i.e. estimating end of on thresholds 67-80 in P2-4 and end of refreshing session in $\mathrm{P} 8-10)\left(\beta=0.43, t_{(9)}=4.112, p=.003, \mathrm{R}^{2}=0.68, F_{(1,9)}=16.900, p=.003\right)$ (Figure 5B).

To further explore the relationship between the learning index and the position specificity index, we correlated the position specificity index with the learning indices from session 1-5, session 6-10, and session 11-15, referred to as the early, intermediate and late learning index, respectively. While the early training index (Figure 6A) did not learning significantly correlate with the position specificity index $\left(r=.58, R^{2}=0.33, p=.081\right)$, there was a significant correlation for intermediate (Figure 6B) and late learning indices (Figure 6C) (intermediate: $r=.65$, $R^{2}=0.42, p=.042$; late: $r=.84, R^{2}=0.70, p=.002$ ), with the late learning index based on a comparison between thresholds 41-44 with thresholds 56-60. When basing the end of late learning in participants P2-4 and P8-10 on the additional asymptotic data (Figure 6D), the correlation between position specificity index and late learning index was even stronger $\left(r=.93, R^{2}=0.87, p<.001\right)$. This indicates that the magnitude of intermediate and late asymptotic learning contributed to the degree of position specificity. A model combining early, intermediate and late learning as predictors was precluded by multicollinearity (intermediate and late learning: $r=0.94)$. Therefore separate models for each of the three predictors were calculated (using the 60-threshold training data). Early learning was not a significant factor for predicting position specificity $\left(\beta=0.56, t_{(9)}=1.999, p=.081\right)$, whereas intermediate and late learning significantly affected position specificity (intermediate: $\beta=0.47, t_{(9)}=2.419, p=.042$; late: $\beta=0.53, t_{(9)}=4.365, p=.002$ ). Intermediate and late learning explained a significant proportion of variance in position specificity (intermediate: $R^{2}=0.42, F_{(1,9)}=5.850, p=.042$; late: $R^{2}=$ $\left.0.70, F_{(1,9)}=19.100, p=.002\right)$. Since in intermediate and late learning indices, there was one data point that qualified as an outlier according to a $1.5 \mathrm{IQR}$ criterion (P2), 
we used a robust regression to see how that data point influenced the estimates. Beta weights from robust regression were almost identical (early: $\beta=0.55, t_{(9)}=1.795, p=$ .110 ; intermediate: $\beta=0.47, t_{(9)}=2.215, p=.058$; late: $\left.\beta=0.52, t_{(9)}=3.967, p=.004\right)$, indicating a negligible contribution of outliers to the OLS regression analysis. When including the training data from extra thresholds in six participants (P2-4 and P8-10) into calculations of the late learning index, the overall picture remained the same $\left(\beta=0.51, t_{(9)}=7.385, p<.001 ; R^{2}=0.87, F_{(1,9)}=54.500, p<.001\right)$. We also correlated a generalization asymmetry index (see Methods) with the learning index to assess the possibility that asymmetric generalization, which was slightly stronger
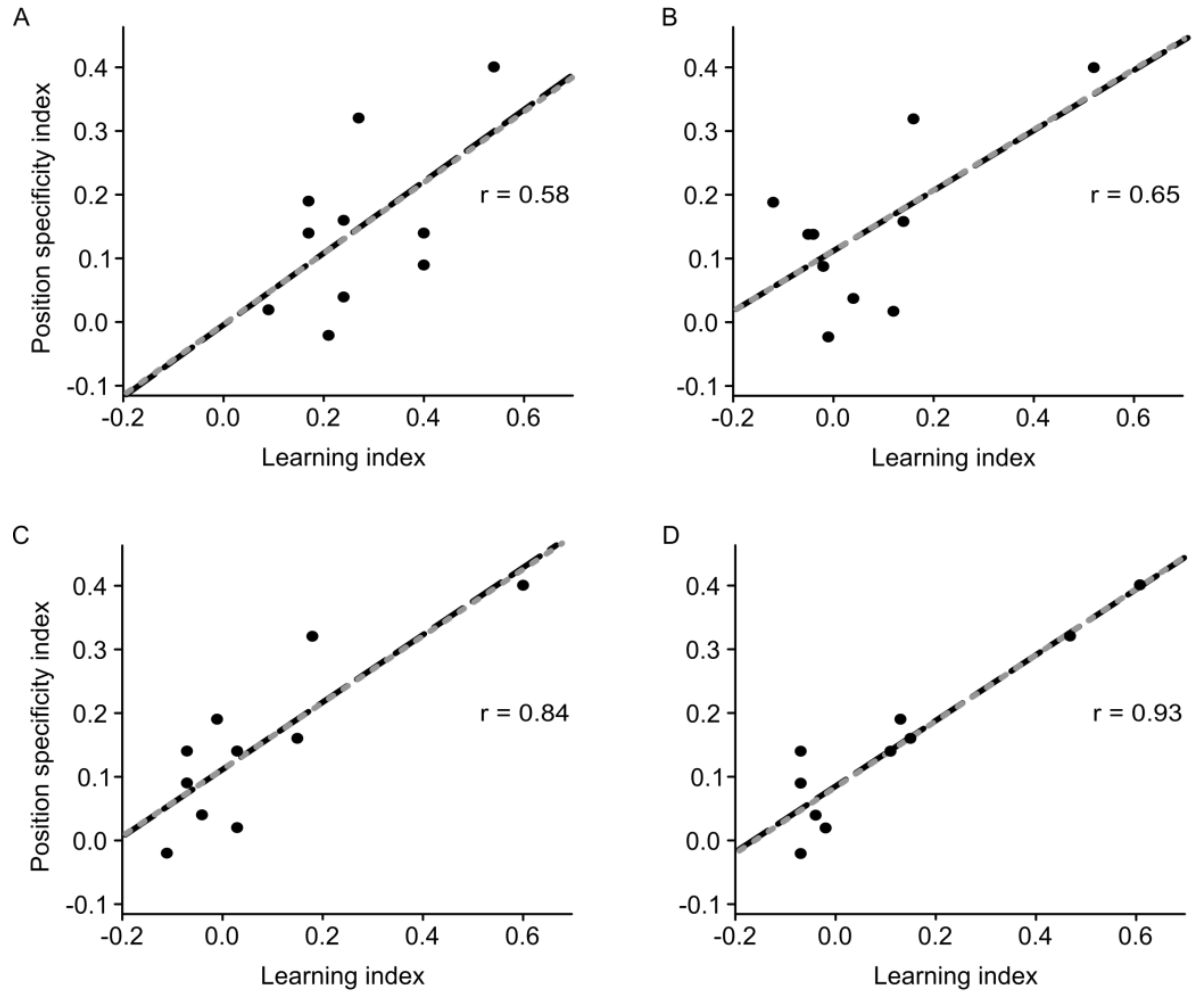

Figure 6. Scatterplot of position specificity and early $(\boldsymbol{A})$, intermediate $(\boldsymbol{B})$, and late learning indices either based on 60 thresholds learning data $(\boldsymbol{C})$ or on additional asymptotic data for participants P2-4 and P8-10 $(\boldsymbol{D})$. The black dashed line results from an ordinary least square regression, whereas the grey dotted line was fitted with betas obtained by using robust regression 
towards the periphery, could be predicted by the learning index. The learning index had no predictive value for asymmetric generalization as shown by a non-significant slope $(p=.891)$. The results were similar when taking early, intermediate and late learning indices or including additional asymptotic data (all $p$-values > .725).

\section{Orientation specificity and training indices}

The orientation specificity effects, just as position specificity effects were rather small compared to total learning. As for position specificity, we calculated and compared training and orientation specificity indices. A paired sample t-test confirmed that the training index $(\mathrm{T}=0.40)$ was larger than the orientation specificity index $(\mathrm{OS}=0.25)\left(t_{(9)}=-5.487, p<0.001\right)$. Hence, overall, the difference in threshold performance between trained $\left(135^{\circ}\right)$ and untrained $\left(45^{\circ}\right)$ orientations was $\sim 1.5$ times smaller than the difference in threshold performance between the beginning and end of learning $(\mathrm{T} / \mathrm{OS}=1.71)$. This analysis was limited to progress made in the first 60 thresholds (as shown in Figure 3). When including all available data (extra thresholds 61-80 in P2-4 and refresher thresholds in P8-10), the ratio T/OS was 1.78.

Using the data from the first 60 thresholds in all participants, we then tested whether the training index was related to the orientation specificity index in a similar way as the position specificity index, and found a positive correlation $\left(r=.70, \mathrm{R}^{2}=\right.$ $0.49, p=.023)$. Similarly to the position specificity index, the more participants improved during the training phase, the greater the orientation specificity effect was (Figure 7A). The training index significantly predicted orientation specificity $(\beta=$ $\left.0.40, t_{(9)}=2.795, p=.023\right)$ and also explained a significant proportion of variance in position specificity $\left(\mathrm{R}^{2}=0.49, F_{(1,8)}=7.81, p=.023\right)$. Robust regression yielded a slightly lower beta weight $\left(\beta=0.39, t_{(9)}=2.413, p=.042\right)$ indicating that outliers did not drive the OLS regression results strongly. This pattern of results was confirmed when including all available training data in all participants (i.e. estimating end of learning on thresholds $67-80$ in P2-4 and end of refreshing session in P8-10) ( $\beta=$ $0.43, t_{(9)}=3.930, p=.004, \mathrm{R}^{2}=0.66, F_{(1,9)}=15.40, p=.004$ ) (Figure 7B).

To further explore the relationship between learning index and orientation specificity index, we also correlated the orientation specificity index with the early, intermediate and late learning indices. While the early (Figure 8A) and intermediate (Figure 8B) training index did not correlate with the position specificity index 
significantly (early: $\mathrm{r}=.35, \mathrm{R} 2=0.12, \mathrm{p}=.324$; intermediate: $\mathrm{r}=57, \mathrm{R} 2=0.33, \mathrm{p}=$ .086), there was a significant correlation for the late learning index (Figure 8C), which was based on a comparison between thresholds 41-44 with thresholds 56-60 ( $\left.r=.76, R^{2}=0.57, p=.011\right)$. When basing the end of late learning in participants P2-4 and P8-10 on the additional asymptotic data (Figure 8D), the correlation between orientation specificity index and late learning index was even stronger $(r=$ $\left..93, R^{2}=0.87, p<.001\right)$. This indicates that the magnitude of learning in the late asymptotic phase contributed to the degree of orientation specificity. Having multicollinearity between intermediate and late learning, only separate models for each of the three predictors were calculated (using the 60-threshold training data). Early and intermediate learning were not a significant factor for predicting orientation specificity (early: $\beta=0.34, t_{(9)}=1.050, p=.324$; intermediate: $\beta=0.41, t_{(9)}$ $=1.961, p=.086$ ), whereas late learning significantly affected orientation specificity $\left(\beta=0.48, t_{(9)}=3.236, p=.011\right)$. Late learning explained a significant proportion of variance in orientation specificity $\left(R^{2}=0.57, F_{(1,9)}=10.60, p<.001\right)$. Beta weights from robust regression were almost identical (early: $\beta=0.34, t_{(9)}=0.950, p=.370$; intermediate: $\beta=0.44, t_{(9)}=1.921, p=.091$; late: $\left.\beta=0.45, t_{(9)}=4.961, p=.001\right)$,
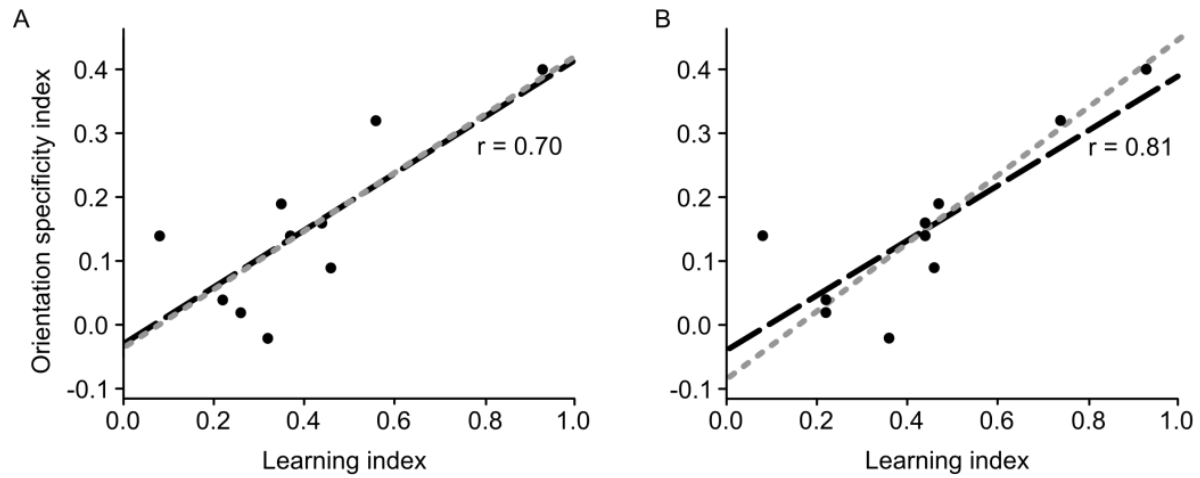

Figure 7. Scatterplot of orientation specificity and learning indices. $\boldsymbol{A}$, Orientation specificity correlated positively with the learning index. $\boldsymbol{B}$, The correlation between orientation specificity and learning index was even stronger when using the complete training data (including extra thresholds and refresher training) for calculation of the learning index. The black dashed line results from an ordinary least square regression, whereas the grey dotted line was fitted with betas obtained by using robust regression. 
indicating a negligible contribution of outliers to the OLS regression analysis. When including the training data from extra thresholds in six participants (P2-4 and P8-10) into calculations of the late learning index, the overall picture remained the same $(\beta=$ $\left.0.51, t_{(9)}=6.728, p<.001 ; R^{2}=0.85, F_{(1,9)}=45.30, p<.001\right)$.

A

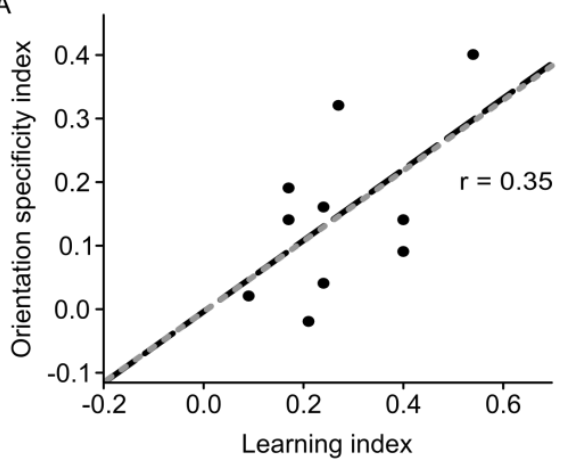

C

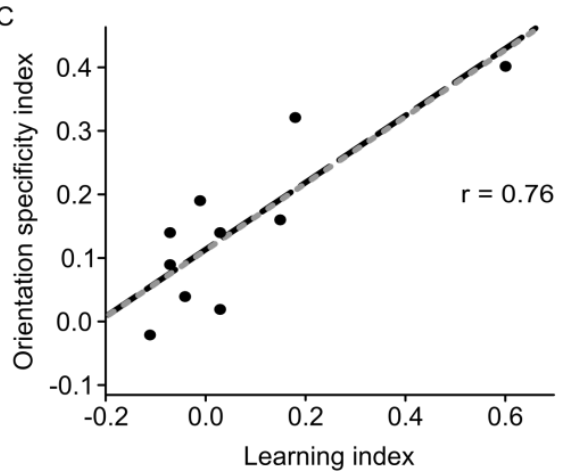

B

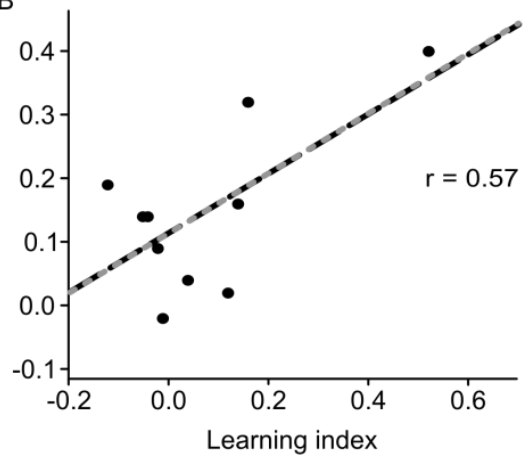

D

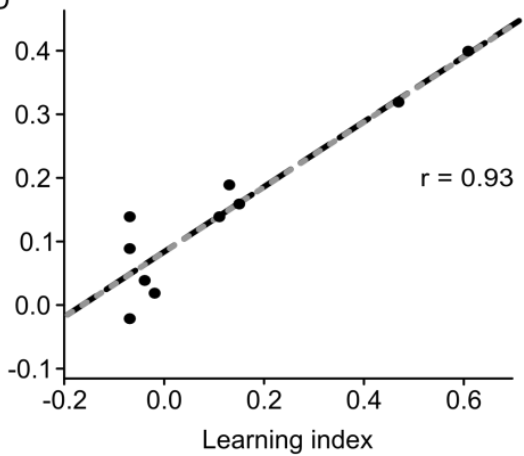

Figure 8. Scatterplot of orientation specificity and early $(\boldsymbol{A})$, intermediate $(\boldsymbol{B})$, and late learning indices either based on 60 thresholds learning data $(\boldsymbol{C})$ or on additional asymptotic data for participants P2-4 and P8-10 (D). The black dashed line results from an ordinary least square regression, whereas the grey dotted line was fitted with betas obtained by using robust regression.

\section{Influence of participants' prior learning history and different training scheme}

As seven of our participants had participated in other perceptual learning studies (in one or sometimes three quadrants other than the one used for within-quadrant position specificity testing), we were interested whether the number of trained 
orientations, quadrants and trials (see Table 1) at which they had been previously trained influenced the observed position specificity effect or the level of the asymptote defined as the last 20 thresholds obtained. Furthermore, the number of trials spent in the present study (see Table 1) differed among participants due to the extra thresholds and refresher thresholds), which was a potential confounder. We therefore correlated the position specificity index, the orientation specificity index, and asymptotic level with these four factors. No significant correlations were found (Table 2). Thus, neither the numbers of trials spent in previous studies, nor the variation among participants in numbers of trials spent in the present study seemed to be determining factors in explaining position specificity.

\begin{tabular}{lccc|c}
\multicolumn{3}{c|}{ previous studies } & present study \\
\cline { 2 - 5 } & $\begin{array}{c}\text { nr. trained } \\
\text { orientations }\end{array}$ & $\begin{array}{c}\text { nr. trained } \\
\text { quadrants }\end{array}$ & $\begin{array}{c}\text { nr. } \\
\text { trials }\end{array}$ & $\begin{array}{c}\text { nr. trials for } \\
\text { training \& refresh }\end{array}$ \\
\hline P1 & 3 & 2 & 15669 & 5352 \\
P2 & 0 & 0 & 0 & 7193 \\
P3 & 0 & 0 & 0 & 6711 \\
P4 & 0 & 0 & 0 & 7146 \\
P5 & 1 & 4 & 17308 & 5127 \\
P6 & 3 & 2 & 15748 & 5356 \\
P7 & 1 & 4 & 16013 & 5302 \\
P8 & 3 & 2 & 15826 & 8068 \\
P9 & 3 & 4 & 36206 & 8690 \\
P10 & 3 & 4 & 36847 & 8711
\end{tabular}

Table 1. Number of trained orientations, trained quadrants, and trials for any previous experiments and number of trials performed during training and refresh sessions for the present experiment. Note that participants P2-4 performed 80 thresholds during training whereas all other participants completed only 60 thresholds. Higher thresholds for P8-10 resulted from refresh sessions for the present study. 


\begin{tabular}{llccc|c} 
& & \multicolumn{3}{c|}{ previous studies } & present study \\
\cline { 3 - 6 } & & $\begin{array}{c}\text { nr. trained } \\
\text { orientations }\end{array}$ & $\begin{array}{c}\text { nr. trained } \\
\text { quadrants }\end{array}$ & $\begin{array}{c}\text { nr. } \\
\text { trials }\end{array}$ & $\begin{array}{c}\text { nr. trials for } \\
\text { training \& refresh }\end{array}$ \\
\hline position & $\mathrm{r}$ & 0.37 & 0.35 & 0.23 & 0.14 \\
specificity & $\mathrm{R}^{2}$ & 0.14 & 0.12 & 0.05 & 0.02 \\
index & $\mathrm{p}$ & 0.295 & 0.324 & 0.531 & 0.707 \\
\hline orientation & $\mathrm{r}$ & 0.31 & 0.30 & 0.08 & 0.48 \\
specificity & $\mathrm{R}^{2}$ & 0.09 & 0.09 & 0.01 & 0.23 \\
index & $\mathrm{p}$ & 0.387 & 0.399 & 0.836 & 0.158 \\
\hline last twenty & $\mathrm{r}$ & 0.23 & 0.51 & 0.31 & 0.41 \\
thresholds & $\mathrm{R}^{2}$ & 0.05 & 0.26 & 0.10 & 0.17 \\
(asymptote) & $\mathrm{p}$ & 0.514 & 0.131 & 0.376 & 0.235
\end{tabular}

Table 2. Correlation, explained variance and $p$-values for different correlations. Upper part shows correlation of position specificity index with number of trained orientations, trained quadrants, and trials of previous studies and number of trials during training for the present study. middle part shows correlations with the same factors. Lower part shows correlations of last tweny thresholds with the same factors mentioned above. 


\section{Discussion}

The study presents three main findings. First, we show that extensive orientation discrimination training at a single location in a visual field quadrant yields significant position and orientation specificity. At the same time, the magnitude of specificity was much smaller than the total range of the training effect achieved in the course of learning. Second, position and orientation specificity indices correlated strongly and positively with training indices derived from intermediate and/or late training phases, indicating that the more improvement was made during later training phases, the larger the specificity of learning was. This is to our knowledge the first evidence strongly linking training effects in the later phases of learning with specificity, in agreements with proposals in older studies (M. Ahissar \& Hochstein, 2004; Karni \& Sagi, 1991, 1993) that asymptotic learning is necessary to achieve position (and stimulus) specific perceptual skill. Third, we found that there was a small tendency for the perceptual skill in orientation discrimination to spread more strongly to more peripheral than to more central positions. This effect was weak and only present in population averages. Indices of training progress in early, middle and late parts of the learning curve were not predictive of an asymmetry of generalization among individual participants. Therefore, we interpret the stronger spread of skill towards peripheral test positions with caution. The finding of enhanced performance at more peripheral test positions was present both for the trained and the untrained (orthogonal) reference orientation. This points to a general effect possibly reflecting increased efficiency in selective attention or read-out (M. Ahissar \& Hochstein, 1993, 1997, 2004; Law \& Gold, 2008, 2009). However, we also found that there was significant position specificity as well as orientation specificity at the trained position. These observations could reflect read-out effects if readout can become highly specific with training, but they may also reflect neuronal tuning changes specific for the trained reference orientation and the trained position in low-level visual areas (Karni \& Bertini, 1997; Karni \& Sagi, 1991, 1993; Raiguel, Vogels, Mysore, \& Orban, 2006; Aniek A. Schoups et al., 1995; Schoups et al., 2001), which are characterized by retinotopy, cortical magnification (Daniel \& Whitteridge, 1961; Gattass, Gross, \& Sandell, 1981; Gattass, Sousa, \& Gross, 1988), and lateral connectivity (Angelucci, Levitt, \& Lund, 2002). The lateral connectivity 
tends to link neurons with similar orientation preferences (Gilbert \& Wiesel, 1989), and could be a substrate for the spread of expertise away from the trained position. A possible interpretation of the finding of slightly lower thresholds for the trained than for the untrained reference orientation in test locations other than the trained $6^{\circ}$ position is that lateral spreading is stronger for the trained than for the untrained reference orientation. However, in general, the pattern of spreading of expertise from the trained to other positions, and from the trained to the non-trained reference orientation is compatible with mechanisms within low-level areas as well as with contributions from read-out interacting with low-level areas.

The variation in position specificity or orientation specificity among participants in our study was not correlated significantly with the variation in number of training trials among participants. To interpret this finding, it is useful to compare the numbers of trials used in our study with prior studies. In our experiment, participants were trained over a period of ten to fifteen sessions and completed a total of 60-80 staircases which amounted to total training trial numbers ranging between 5127 and 8711 trials when including the extra trials in P2-4 and the refresher trials in P8-10 (see Table 1). Jeter et al. (2010) report that shorter training (i.e., up to 2496 trials) resulted in transferable performance levels, whereas extensive training (i.e., 4992 or 7488) resulted in partial specificity. Given the overall number of trials (mean number of trials 6766) it is therefore fully in line with Jeter et al.'s results that we see specificity in our data. Another factor that can play a role is the number of trials within a session, which, when sufficiently large could lead to adaptation effects that could enhance the specificity of learning (Harris et al., 2012). Aberg et al. (2009) observed generalization in a chevron task with 400 trials per session and specificity with 800 trials per session with the overall amount of training being identical. In our study the number of trials per session was rather low for the seven participants doing 15 training sessions (mean of 354 trials/session) and higher for three participants (P2-4) doing ten training sessions (mean of 702 trials/session). Situated in the entire group of ten participants, the specificity index was largest in $\mathrm{P} 2$, sixth largest in $\mathrm{P} 3$, and third largest in $\mathrm{P} 4$. Thus the specificity indices for $\mathrm{P} 3$ and P4 fell within the range of the other participants, and outlier analysis showed that the index for P2 fell within the normal range. It therefore seems unlikely that there were 
strong additional adaptation effects in P2-4 that would have led to greater position specificity in those subjects, and hence in our hands, sessions with roughly 350 or roughly 700 trials per session can both lead to specificity. However, Aberg et al. (2009) only had 1600 trials in total, so our finding of partial specificity to the trained position, even with rather low trial numbers per session for seven participants, might be explained by the more extensive total amount of training in our study, which continued until participants reached an asymptotic performance level. Recently, Hung and Seitz (2014) showed in a Vernier discrimination task that by using a single but very long staircase, which increased the relative amount of training at threshold, position specificity was observed while training for a comparable number of trials in eight separate staircases, each initiated above threshold, led to complete generalization. Although the result was not as clear cut for orientation discrimination, there was still greater transfer for training using multiple short staircases in contrast to a single staircase (Hung \& Seitz, 2014). We used four to eight staircases per session during the training phase; however we trained for ten or more sessions (yielding between 60 and 99 thresholds per participant depending on the training scheme) and also adjusted the start level of the staircase to be the average threshold level of the previous session. In addition, participants were trained for at least ten and a maximum of 19 sessions, resulting in a prolonged period of training at an asymptotic threshold level towards the end of the training. This might explain why our results were comparable to the single staircase condition in the study of Hung and Seitz (2014). Our finding in the present study that the number of trials does not play a role in predicting differences in specificity among participants suggests that the numbers of trials administered (in total, and per session) are above a certain threshold so that trial numbers in themselves are not a determinant of the magnitude of learning or specificity. Instead, differences in learning and specificity among participants may reflect differences in the capacity for plastic change in their visual systems, rather than differences in induced plasticity due to the possibility that the number of trials given would be sufficient to induce full plasticity in some participants, and not in others. This reasoning is in line with our finding that the magnitude of threshold decrease (especially in the later parts of learning) correlates 
best with position and orientation specificity, and not the number of training trials performed.

Our results are relevant for studies that have been appearing over the last seven years suggesting almost complete generalization of learning in a so-called 'double training' paradigm (Xiao et al., 2008; J.-Y. Zhang et al., 2010). In these studies, a brief period of training in an irrelevant task at a first location, followed by training in a second task in another location, led to generalization of the skill acquired in the second task towards the first location. Based on these findings, it was suggested that the idea of specificity in perceptual skill learning may be altogether incorrect. They suggested instead that visual perceptual learning may be rule-based, and hence generalization would reflect processes taking place independent of low-level properties of low-level visual areas. In this view, there should be full generalization, or generalization should be easily triggered in the context of specific training paradigms (Xiao et al., 2008; J.-Y. Zhang et al., 2010). In the present study, we did find however position and stimulus specificity. This could be considered surprising given the experience of a majority of our participants with similar tasks in visual field positions other than the ones we used in the present experiment. Moreover, the position and orientation specificity tests in the present study showed threshold increases between trained and test positions matching the threshold increase from session 15 to session 4-8, which is approximately where Xiao et al. (2008) and J.-Y. Zhang et al. (2010) ended their training. We suggest, in line with other studies, that there is a strong tendency for generalization in the first parts of the learning curve (Jeter et al., 2010). Hence, Xiao et al.'s (2008) findings can be seen as revealing conditions in which learning transfer can be facilitated in the early phase of learning. It is possible that part of the mechanisms leading to generalization during early learning is rule-based. However, the mechanisms underlying the amount of transfer (i.e., the amount of specificity) in the present study were unrelated to early learning, as indicated by a lack of a correlation in our study between position/orientation specificity and performance improvements in early learning. We therefore suggest that mechanisms and conditions leading to specificity/generalization of learning are different for early and asymptotic learning. The slight trend towards more generalization in the peripheral direction may suggest that mechanisms that lead to 
specificity in later phases of learning are in part localized in or interact with lowlevel visual areas. It should be noted that the training in the present study was still fairly limited, and that training over many months can lead to thresholds much lower than obtained in our study (e.g., see Schoups et al., 1995; 2001). Future studies of specificity in perceptual skill learning may benefit from comparing the properties of generalization between short periods of training (e.g., 7 sessions) and much longer periods of training (e.g., 30 days). 


\section{Acknowledgements}

GL was supported by a grant from the Netherlands Organization for Scientific Research to the FPN Graduate School for Cognitive and Clinical Neuroscience (NWO, 022.001.036). PDW was supported by a grant from the Netherlands Organization for Scientific Research (NWO VICI grant, 453.04.002). We thank Bert Jans for help with stimulus design. 


\section{References}

Aberg, K. C., Tartaglia, E. M., \& Herzog, M. H. (2009). Perceptual learning with chevrons requires a minimal number of trials, transfers to untrained directions, but does not require sleep. Vision Research, 49(16), 2087-2094.

Ahissar, E., Vaadia, E., Ahissar, M., Bergman, H., Arieli, A., \& Abeles, M. (1992). Dependence of cortical plasticity on correlated activity of single neurons and on behavioral context. Science, 257(5075), 1412-1415.

Ahissar, M., \& Hochstein, S. (1993). Attentional control of early perceptual learning. Proceedings of the National Academy of Sciences, 90(12), 5718-5722.

Ahissar, M., \& Hochstein, S. (1996). Learning pop-out detection: Specificities to stimulus characteristics. Vision Research, 36(21), 3487-3500.

Ahissar, M., \& Hochstein, S. (1997). Task difficulty and the specificity of perceptual learning. Nature, 387(6631), 401-406.

Ahissar, M., \& Hochstein, S. (2004). The reverse hierarchy theory of visual perceptual learning. Trends in Cognitive Sciences, 8(10), 457-464.

Ahissar, M., Laiwand, R., Kozminsky, G., \& Hochstein, S. (1998). Learning pop-out detection: Building representations for conflicting target-distractor relationships. Vision Research, 38(20), 3095-3107.

Angelucci, A., Levitt, J. B., \& Lund, J. S. (2002). Anatomical origins of the classical receptive field and modulatory surround field of single neurons in macaque visual cortical area v1. Progress in brain research, 136, 373-388.

Ball, K., \& Sekuler, R. (1981). Adaptive processing of visual motion. Journal of Experimental Psychology: Human Perception and Performance, 7(4), 780-794.

Censor, N., \& Sagi, D. (2009). Global resistance to local perceptual adaptation in texture discrimination. Vision Research, 49(21), 2550-2556.

Crist, R. E., Kapadia, M. K., Westheimer, G., \& Gilbert, C. D. (1997). Perceptual learning of spatial localization: Specificity for orientation, position, and context. Journal of Neurophysiology, 78(6), 2889-2894.

Daniel, P., \& Whitteridge, D. (1961). The representation of the visual field on the cerebral cortex in monkeys. The Journal of Physiology, 159(2), 203-221.

Fahle, M., Edelman, S., \& Poggio, T. (1995). Fast perceptual learning in hyperacuity. Vision Research, 35(21), 3003-3013.

Fiorentini, A., \& Berardi, N. (1980). Perceptual learning specific for orientation and spatial frequency. Nature, 287(5777), 43-44. 
Gattass, R., Gross, C., \& Sandell, J. (1981). Visual topography of v2 in the macaque. Journal of Comparative Neurology, 201(4), 519-539.

Gattass, R., Sousa, A., \& Gross, C. (1988). Visuotopic organization and extent of v3 and v4 of the macaque. The Journal of Neuroscience, 8(6), 1831-1845.

Ghose, G. M., Yang, T., \& Maunsell, J. H. R. (2002). Physiological correlates of perceptual learning in monkey v1 and v2. Journal of Neurophysiology, 87(4), 1867 1888.

Gilbert, C. D., \& Wiesel, T. N. (1989). Columnar specificity of intrinsic horizontal and corticocortical connections in cat visual cortex. The Journal of Neuroscience, 9(7), 2432-2442.

Harris, H., Gliksberg, M., \& Sagi, D. (2012). Generalized perceptual learning in the absence of sensory adaptation. Current Biology, 22(19), 1813-1817.

Hochstein, S., \& Ahissar, M. (2002). View from the top: Hierarchies and reverse hierarchies in the visual system. Neuron, 36(5), 791-804.

Hung, S.-C., \& Seitz, A. R. (2014). Prolonged training at threshold promotes robust retinotopic specificity in perceptual learning. The Journal of Neuroscience, 34(25), 8423-8431.

Jeter, P. E., Dosher, B. A., Liu, S.-H., \& Lu, Z.-L. (2010). Specificity of perceptual learning increases with increased training. Vision Research, 50(19), 1928-1940.

Jeter, P. E., Dosher, B. A., Petrov, A., \& Lu, Z.-L. (2009). Task precision at transfer determines specificity of perceptual learning. Journal of Vision, 9(3), 1-13.

Karni, A., \& Bertini, G. (1997). Learning perceptual skills: Behavioral probes into adult cortical plasticity. Current Opinion in Neurobiology, 7(4), 530-535.

Karni, A., \& Sagi, D. (1991). Where practice makes perfect in texture discrimination: Evidence for primary visual cortex plasticity. Proceedings of the National Academy of Sciences, 88(11), 4966-4970.

Karni, A., \& Sagi, D. (1993). The time course of learning a visual skill. Nature, 365(6443), 250-252.

Kehrer, L. (1989). Central performance drop on perceptual segregation tasks. Spatial vision, 4(1), 45-62.

Kehrer, L. (1997). The central performance drop in texture segmentation: A simulation based on a spatial filter model. Biological Cybernetics, 77(4), 297-305.

Kehrer, L., \& Meinecke, C. (2006). A'first stage'central performance drop in a gabor luminance-modulation detection task. Spatial vision, 19(5), 427-4371. 
Kuai, S.-G., Zhang, J.-Y., Klein, S. A., Levi, D. M., \& Yu, C. (2005). The essential role of stimulus temporal patterning in enabling perceptual learning. Nature Neuroscience, 8(11), 1497-1499.

Law, C.-T., \& Gold, J. I. (2008). Neural correlates of perceptual learning in a sensory-motor, but not a sensory, cortical area. Nature Neuroscience, 11(4), 505513.

Law, C.-T., \& Gold, J. I. (2009). Reinforcement learning can account for associative and perceptual learning on a visual-decision task. Nature Neuroscience, 12(5), 655663.

Raiguel, S., Vogels, R., Mysore, S. G., \& Orban, G. A. (2006). Learning to see the difference specifically alters the most informative v4 neurons. The Journal of Neuroscience, 26(24), 6589-6602.

Schoups, A. A., Vogels, R., \& Orban, G. A. (1995). Human perceptual learning in identifying the oblique orientation: Retinotopy, orientation specificity and monocularity. The Journal of Physiology, 483(Pt 3), 797-810.

Schoups, A. A., Vogels, R., Qian, N., \& Orban, G. A. (2001). Practising orientation identification improves orientation coding in v1 neurons. Nature, 412(6846), 549553.

Sereno, M., Dale, A., Reppas, J., Kwong, K., Belliveau, J., Brady, T., . . . Tootell, R. (1995). Borders of multiple visual areas in humans revealed by functional magnetic resonance imaging. Science, 268(5212), 889-893.

Wang, R., Zhang, J.-Y., Klein, S. A., Levi, D. M., \& Yu, C. (2012). Task relevancy and demand modulate double-training enabled transfer of perceptual learning. Vision Research, 61(0), 33-38.

Wang, X., Zhou, Y., \& Liu, Z. (2013). Transfer in motion perceptual learning depends on the difficulty of the training task. Journal of Vision, 13(7), 1-9.

Watanabe, T., Nanez, J. E., Koyama, S., Mukai, I., Liederman, J., \& Sasaki, Y. (2002). Greater plasticity in lower-level than higher-level visual motion processing in a passive perceptual learning task. Nature Neuroscience, 5(10), 1003-1009.

Wetherill, G. B., \& Levitt, H. (1965). Sequential estimation of points on a psychometric function. British Journal of Mathematical and Statistical Psychology, 18(1), 1-10.

Xiao, L.-Q., Zhang, J.-Y., Wang, R., Klein, S. A., Levi, D. M., \& Yu, C. (2008). Complete transfer of perceptual learning across retinal locations enabled by double training. Current Biology, 18(24), 1922-1926. 
Zhang, J.-Y., Zhang, G.-L., Xiao, L.-Q., Klein, S. A., Levi, D. M., \& Yu, C. (2010). Rule-based learning explains visual perceptual learning and its specificity and transfer. The Journal of Neuroscience, 30(37), 12323-12328.

Zhang, T., Xiao, L.-Q., Klein, S. A., Levi, D. M., \& Yu, C. (2010). Decoupling location specificity from perceptual learning of orientation discrimination. Vision Research, 50(4), 368-374. 


\section{CHAPTER 3}

Limited transfer of orientation discrimination performance after pre-test and masked exposure

Lange, G. \& De Weerd, P. (submitted). Limited transfer of orientation discrimination performance after pre-test and masked exposure 


\begin{abstract}
There is increasing evidence that complete transfer of skill learning can be achieved by a brief pre-test, training-plus-exposure, or a double training paradigm (Xiao et al., 2008; J.-Y. Zhang et al., 2010; Zhang, Xiao, Klein, Levi, \& Yu, 2010). Here, we compared the generalization of perceptual learning in a training location towards four other test locations, each exposed to a different type of treatment. In one test location, there was brief pre-testing in the first session prior to onset of learning in the training location. Two other locations were exposed to masked stimuli similar or identical to concurrently presented stimuli in the training location. In the fourth test location, no stimuli were presented prior or during training. In the present study, we not only tested generalization of training to the test locations immediately after completing training in the training location, we also investigated whether any advantage in the pre-tested or exposed test locations extended over an entire learning curve in comparison with the unstimulated test location. Hence, after 15 sessions of an orientation discrimination task at one training location, participants completed a second training phase of 15 sessions on the four testing positions. Compared to the untrained/unexposed test location, we found small advantages (i.e. limited generalization) for pre-tested and exposed test locations. However, these advantages were small, and were limited to the first few training sessions.
\end{abstract}




\section{Introduction}

Perceptual learning is defined as the acquisition of a perceptual skill over time. This learning process is characterized by very fast improvements during early training and subsequent learning that gradually slows down until reaching asymptotic performance levels (Karni, 1996; Karni \& Bertini, 1997; Karni et al., 1998; Karni \& Sagi, 1993). Once a skill is learned performance is retained for extended periods without further training (De Weerd, Pinaud, \& Bertini, 2006). Especially during this asymptotic learning phase, learning has been reported to become specific to stimulus characteristics (Fahle, 1997; Karni \& Bertini, 1997; Karni et al., 1995; Karni \& Sagi, 1991; Schoups, Vogels, \& Orban, 1995; Schwartz, Maquet, \& Frith, 2002).

Recently a series of experiments have cast doubt upon the idea that specificity is a defining characteristic of perceptual learning (Wang, J.-Y. Zhang, Klein, Levi, \& Yu, 2012; Xiao et al., 2008; J.-Y. Zhang et al., 2010; T. Zhang et al., 2010). Xiao et al. (2008) showed that a double-training procedure consisting of feature and location training resulted in complete transfer of learning across retinal locations. Subsequently, a training-plus-exposure procedure was introduced (J.-Y. Zhang et al., 2010) where after initial training, an irrelevant task was performed at the transfer position on another stimulus feature whereas the stimulus feature of interest was simply exposed (and assumed to be unattended). Again, this experimental procedure resulted in complete transfer. Wang et al. (2012) extended these finding by showing that task relevance and demand modulate the amount of transfer obtained in a double-training procedure. While a brief pre-test could bring about complete transfer (T. Zhang et al., 2010), passive exposure alone was insufficient to elicit transfer (Wang et al., 2012).

Regarding the finding that pre-training can bring about full transfer from a trained location to the pre-trained location, it is interesting that Hung and Seitz (2014) recently reported that prolonged training at threshold in the trained location during a double-training procedure reestablished location specificity during generalization testing. Hence, the full transfer in the double training studies might be due to lack of sufficient training. Regarding the finding in (Wang et al., 2012) that passive exposure is insufficient to bring about transfer also some questions can be raised. There is evidence that repeated stimulus repetition leads to adaptation (for a 
review, see Grill-Spector, Henson, \& Martin, 2006), a state that has been associated with increased discrimination capabilities (Regan \& Beverley, 1985). Adaptation has also been linked with skill learning and recently it has been shown that by removing adaptation complete generalization can be achieved in a texture discrimination task (Harris, Gliksberg, \& Sagi, 2012). Moreover, it has also been shown that repeated exposure to unattended stimuli can result in perceptual learning (Gutnisky, Hansen, Iliescu, \& Dragoi, 2009; Watanabe, Náñez, \& Sasaki, 2001). In addition, a study by Tsushima, Seitz and Watanabe (2008) suggested that passively exposed stimuli of which the observer is aware during performance of another task may be suppressed by attention, so that an unconscious form of exposure might provide a more effective tool to test a potential contribution to learning (Seitz \& Watanabe, 2003).

In the light of the conflicting literature, we performed a Gabor orientation discrimination learning experiment with three aims. First, we wanted to replicate the finding of Hung and Seitz (2014) that there is no longer substantial generalization to a pre-tested location when training at threshold in the trained location is sufficiently prolonged. Second, we wanted to test whether exposure to Gabor stimuli, when ignored and masked, and presented for a significantly greater number of trials than for the exposure stimuli in Wang et al.'s (2012) study, would trigger generalization from a location where prolonged training took place. We used two passive exposure conditions to which transfer was tested: In one condition, participants were exposed to masked, unattended stimuli that were identical to those used in the actively trained task, such that visual feedback on correctness in that task in the trained location applied correctly to the unseen stimuli in the exposure location. In the other exposure condition, the unseen oriented stimuli were randomized in orientation (details in Methods) so that the feedback signal became irrelevant and any passive learning would only reflect the effects of exposure. We anticipated that compared to Wang et al. (2012), these procedures would lead to more significant transfer from a trained location to one or possibly both of the masked exposure test locations. Third, for all the different test locations, we wished to verify how robust the advantage would be after pre-testing or after masked exposure compared to a location where no stimuli had been shown. Whereas in all prior studies generalization testing was limited to a single session, in the present study prolonged training was performed in 
all test positions to verify the robustness of any advantages afforded by masked exposure and pretesting. 


\section{Methods}

\section{Participants}

Eight participants (7 female, mean age 22.27, $S D$ 1.41) naïve to the purpose of the study participated in the experiment and the control condition. All participants had normal or corrected-to normal visual acuity. Informed, written and verbal consent was obtained according to the Helsinki Declaration, after full information about all procedures and the right to withdraw participation at any time. All procedures were approved by the local Ethical Committee of the Faculty of Psychology and Neuroscience (ECP). For their participation in the study participants received either monetary reward or credits to fulfill course requirements.

\section{Task, Stimuli, and Apparatus}

Participants performed an orientation discrimination task with a never presented oblique orientation as reference stimulus. They indicated the direction of the orientation offset of a Gabor stimulus from the oblique reference by pressing either the left or right arrow key for counterclockwise and clockwise rotations respectively (Figure 1A). Each trial started with a 950ms window in which participants had the opportunity to initiate fixation. Without fixation, this waiting period was restarted. Successful initiation of fixation was followed by a $300 \mathrm{~ms}$ period in which steady fixation was required (within $1.5^{\circ}$ from the fixation point) to trigger stimulus presentation. Stimulus presentation was $33 \mathrm{~ms}$ and was followed immediately in some conditions by a mask of $49 \mathrm{~ms}$. Fixation errors during pre-stimulus and during stimulus period led to abortion of the trial, which was then presented again at a random moment later on in the threshold measurement. The response window started from the beginning of stimulus onset and was $1500 \mathrm{~ms}$ long. Upon response the fixation spot changed to green or red and remained visible for $250 \mathrm{~ms}$ after which the response window was terminated and the next trial started. The inter-trialinterval depended on the speediness of responses and on how quickly participants regained fixation.

The Gabor stimuli used showed small clockwise or counterclockwise deviations from $135^{\circ}$ (2.37 cycles/degree spatial frequency, 50\% Michelson contrast, $3^{\circ}$ diameter, $8^{\circ}$ eccentricity, $33 \mathrm{~ms}$ presentation time, average luminance 56 
$\mathrm{cd} / \mathrm{m} 2$ ). The fixation dot was a small white dot of $0.2^{\circ}$ diameter. The mask was the same size as the Gabor stimuli. It was equiluminant with the Gabor stimuli and the background, and consisted of small pixels $\left(0.05^{\circ}\right.$ by $\left.0.05^{\circ}\right)$, randomly positioned, and with varying shades of gray, white, and black. The mask was convolved with a Gaussian filter $\left(\mathrm{HWHM}=0.34^{\circ}\right)$. The timing and spatial characteristics of the mask were piloted in the two authors. We deliberately chose to use a non-optimal stimulus termination asynchrony of 49ms (Macknik \& Livingstone, 1998) and a non-optimal spatial frequency (much higher than in the Gabor) to avoid completely preventing processing of the masked stimulus (Bruchmann, Breitmeyer, \& Pantev, 2010), as we reasoned a minimum of processing would be necessary to permit a possible learning effect. In addition, we did not individually calibrate the masks for each participant, as this would have involved presenting the Gabor stimuli prior to the experiment, which would have induced awareness of the Gabor stimuli in the masked conditions, or would have constituted a form of pre-testing that could have interfered with the planned experiment.

Participants were placed in a dimly lit room; their head was supported by a chin and head rest keeping eye-screen distance constantly at $57 \mathrm{~cm}$. Visual stimuli were displayed on a 19" Samsung SyncMaster 940BF LCD monitor (Samsung, Seoul, South Korea; $60 \mathrm{~Hz}$ refresh rate, $1280 \times 1024$ resolution). The screen was covered by a gray cardboard with an oval aperture so that the screen borders were not visible to participants and thus could not be used as reference for the orientation discrimination task. Fixation control was monitored with a Viewpoint Eyetracker v.2.8.3 (Arrington Research, Inc., Scottsdale, Arizona, USA; 60Hz sampling rate). Stimulus presentation and response recording was performed by Cortex v.5.9.6 (NIH freeware for psychophysical and neurophysiological experimentation).

\section{Training protocol}

Over the course of the experiment, participants were trained in five different, equally spaced positions at $8^{\circ}$ eccentricity (Figure 1B). In the first training phase, participants underwent extensive training in a single position resulting in asymptotic learning. Over the course of 15 sessions, participants performed the orientation discrimination task on a $135^{\circ}$ oblique Gabor stimulus at $8^{\circ}$ eccentricity along a $0^{\circ}$ 
polar angle line (referred to as baseline learning; Figure 1B, left panel). During the entire first learning phase, masked Gabor stimuli oriented around a $135^{\circ}$ reference orientation were presented at two additional positions (masked exposure); at one position the stimuli had systematically the same orientation off-set from the reference orientation as the stimulus in the trained position (masked exposure, matching offset); at the other position the orientation off-set matched, but the leftright deviation from the reference was random (masked exposure, randomized offset direction). In addition, during the first session, participants also performed a block of the orientation discrimination task at one other position (4 staircases). This location is referred to as the pre-tested location. A fifth position was left unstimulated during the first learning phase, to which we refer as the unstimulated position. The order of baseline learning, and pretest was counterbalanced over participants in the first session. In all sessions, baseline learning was accompanied with masked presentation of Gabors in the two masked exposure locations. The four different treatments of the four test locations are referred to as four different types of 'preconditioning'.

After completion of the first training phase, participants were asked to rate visibility (5- point scale) of the masked, unattended stimuli participants were exposed to. Two participants reported not seeing anything at the positions used for passive exposure, five participants indicated they had only noticed the mask, and one participant reported to have noticed an oriented Gabor stimulus. Hence in seven of eight participants, there was no awareness of the Gabor stimulus. Altogether, this shows that in the large majority of trials, there was no awareness of the passive stimuli.

During the second training phase, participants were trained for another 15 sessions on the four positions where no training had yet taken place. That is, secondphase training took place in one position that had been unstimulated during the entire first learning phase, in a second position where masked Gabor stimuli had been shown which had identical orientations as in the baseline-trained position, in a third position where masked Gabor stimuli were shown with random direction of the orientation off-set, and on a fourth position where there had been one-time testing during the first session of the first training phase (Figure1B). 
The different positions were counterbalanced over participants in such a way that the two masked exposure conditions were shown in one visual hemifield, whereas the

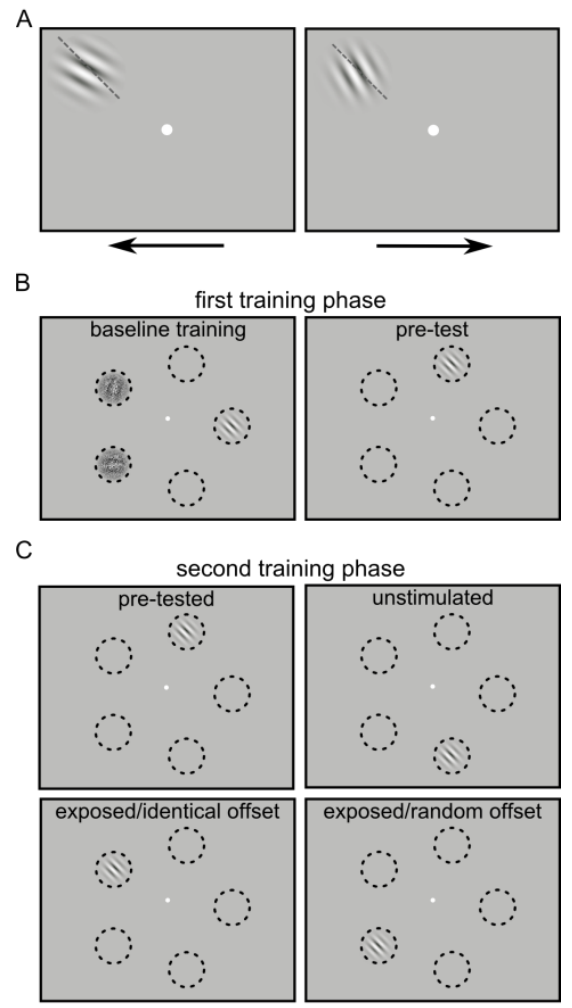

Figure1. Orientation discrimination task (ODT) and experimental design. A, Stimuli and task. Thresholds were determined using an identification design, with left key responses for orientations with counterclockwise off-set from a $135^{\circ}$ reference orientation (dashed line, not shown to participants), and right key responses for orientations with clockwise orientation offset. Stimuli are enlarged for illustration purpose. B, First training phase: Participants were trained on a single position for 15 sessions, referred to as baseline training. On two other positions masked, oriented stimuli were shown $\left(135^{\circ}\right)$, one of them with random, the other with identical orientation off-sets as the stimulus at the trained position. During the first session, there was one-time additional pre-test on another position. $\mathbf{C}$, Second training phase: Subsequent training on the remaining four positions for 15 sessions. Dashed circles indicate the five different stimulus positions and were not shown to participants. The position for baseline training was fixed, all other positions counterbalanced across participants. 
pre-tested location was in the other hemifield together with the previously unstimulated position. Within one hemifield the upper and lower quadrant were also counterbalanced over participants.

During both training phases four staircases were done per position, which accumulated to a total of 60 staircases per position in a total of 15 sessions. At least three sessions were completed within a week, and within each training phase no weeks without training were permitted. The 30 sessions took place at the same time of the day (variations up to one hour were accepted only exceptionally). After finishing the main experiment, all participants participated in an additional control session. Participants performed three staircases of the orientation discrimination task at five positions intermediate to the original stimulus positions and at the same eccentricity (Figure 2, referred to as control positions). We compared these thresholds at intermediate positions with those obtained in the four pre-conditioning positions (unstimulated, pre-tested, and two masked exposure positions). In each intermediate position, three staircases were measured in each participant (with order of positions randomized over participants). For performance in the four predefined

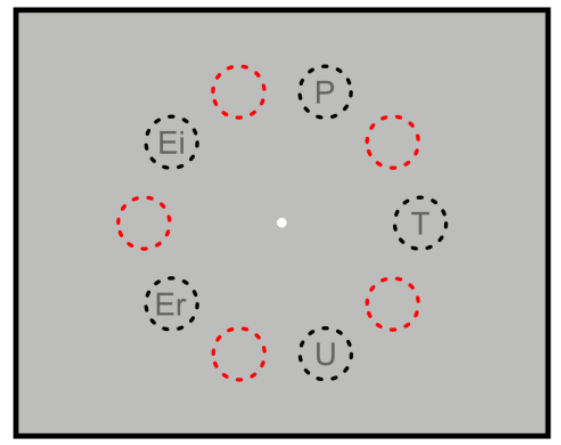

Figure 2. Stimulus positions for the control session. Blacked dashed circles indicate the five original stimulus positions with the letter indicating the condition presented at that position in this example. P refers to 'pre-tested', Ei refers to 'masked-exposure/identical offset direction', Er to 'masked exposure/random offset direction', U refers to 'unstimulated'. Red dashed circles indicate the intermediate stimulus positions used in the two final control sessions. In each of those five intermediate positions participants completed three staircases. 
positions, we took the first three staircases of the last session of the second training phase (thereby keeping numbers of thresholds identical across all positions).

\section{Data and Statistical analyses}

Thresholds were measured using a Wetherill \& Levitt (1965) staircase tracking 84\% correct performance with a 4-down-1-up rule. The staircase was automatically terminated either when 14 reversal points were acquired or 120 trials were completed. The threshold was calculated by taking the geometric mean of the last ten reversal points from each staircase. Per session, thresholds for each position were obtained by averaging JNDs of the four staircases. For the control session JNDs were averaged of only three staircases. For the first session, the start level of the staircase was set to $\pm 15^{\circ}$ orientation deviations, for all subsequent sessions the average threshold level of the previous session was taken. Thresholds were transformed using the natural logarithm.

Data of all five positions were analyzed with a repeated-measures ANOVA with factors session and exposure history (empty, pre-tested, masked with identical offsets, or masked with random offsets) to investigate in how far baseline training transferred to any of the exposed positions. In cases where the sphericity assumption was violated (tested with Mauchly's Test of Sphericity), Greenhouse-Geisser correction was applied. Pairwise comparisons were adjusted for multiple pairwise comparisons using Bonferroni correction unless otherwise indicated.

To quantify position specificity we calculated a generalization index similar to the specificity index of Ahissar \& Hochstein (1997)

$$
G I=\frac{T 1_{\text {begin }}-T 2_{t}}{T 1_{\text {begin }}-T 1_{\text {end }}}
$$

where $T l_{\text {begin }}$ and $T l_{\text {end }}$ were the first and last orientation threshold of the first training phase (session 1 and 15). $T 2_{t}$ is the threshold of the session(s) of interest of the second training phase (i.e., session 16, and session 17-19,) for a specific type of preconditioning (i.e., pre-tested, exposed/identical offset, exposed/random offset direction, or unstimulated). Additionally, the same index was also calculated to compare the mean calculated over the first three thresholds of session 30 in all 
transfer positions (referred to as original stimulus positions) with the mean of thresholds averaged over all intermediate positions. 


\section{Results}

\section{Orientation discrimination thresholds for different exposure conditions}

We investigated the effect of brief pre-testing and of two types of masked exposure on generalization of skill acquired in a single fully trained location. In addition, we used a unstimulated condition as a control. Figure 3A shows that in the first training phase, the first session (red dot) revealed equivalent performance in the pre-test location and in the baseline training location. Further training in the baseline location revealed strong learning (black line). In the second training phase, a comparison between the training in the unstimulated and pretested conditions (Figure 3B) and between unstimulated and masked-exposure conditions (Figure 3C) did not reveal large advantages (generalization) in the pre-tested and exposed locations. To explore the data in detail, we started by performing a repeated measures ANOVA with a preconditioning factor (pre-tested, exposure/identical offset, exposure/random offset direction, and unstimulated) and session (16-30) as within-subjects factors. We found that the main effect of preconditioning was not significant $\left(F_{(3,21)}=2.343 ; p=.102\right)$, whereas the main effect of session $\left(F_{(14,98)}=\right.$ 9.714; $p<.001)$ and the interaction $\left(F_{(42,294)}=1.481 ; p=.034\right)$ were significant. This indicated that the differences in thresholds among types of preconditioning depended on session number (Figure 3A-C).

To explore the interaction, we divided the second training phase into three stages. The first stage of interest was session 16 (i.e., first session of second training phase), where we wanted to test initial effects of pre-testing and masked exposure on generalization from the baseline training position to those three conditions, and to the unstimulated control condition, as done in other studies (Wang et al., 2012; T. Zhang et al., 2010). The second stage we considered included sessions 17 to 19 (i.e. second to fourth session of second training phase), during which we wanted to assess short-term robustness of any learning transfer. The third stage consisted of the remaining sessions 20 to 30 (i.e. fifth to fifteenth session of second training phase). For each stage, a separate analysis was performed. For stage one (Figure 3D+E), the repeated measures ANOVA with preconditioning type as within-subject factor revealed a significant main effect of preconditioning $(F(3,21)=5.080 ; \mathrm{p}=.008)$. 
Pairwise comparisons showed that only the difference between pre-tested and unstimulated conditions (Fig 3D) was significant $(\mathrm{t}(7)=4.098, \mathrm{p}=.028)$. All other comparisons (Fig 3E) failed to reach significance (unstimulated vs. exposed/random offset direction: $t_{(7)}=1.883, p=.611$; unstimulated vs. exposed/identical offset: $t_{(7)}=$ $2.938, p=.131$; pre-tested vs. exposed/identical offset: $t_{(7)}=0.980, p>.999$; pre-

A

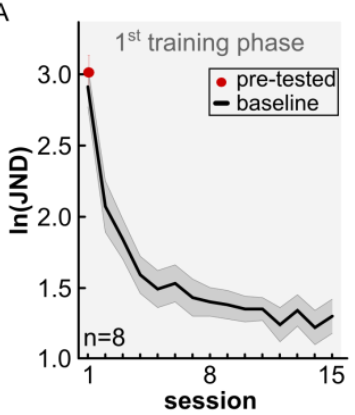

D

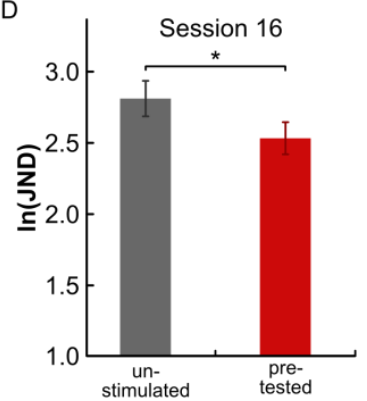

B

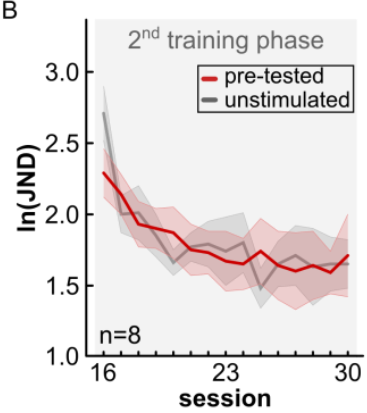

E

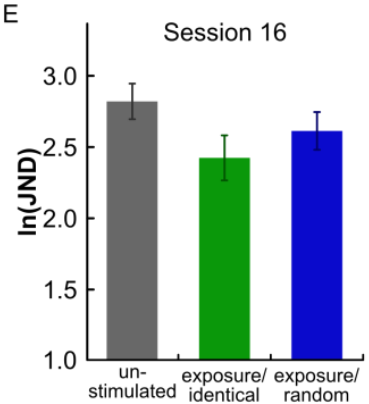

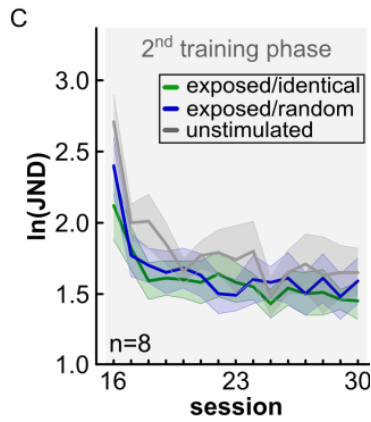

$\mathrm{F}$

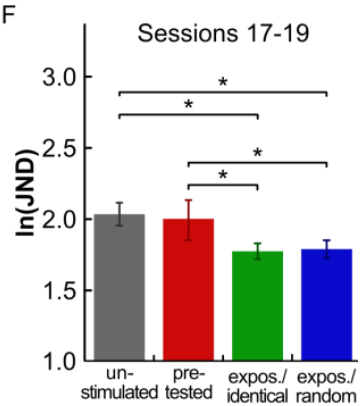

Figure 3. $\boldsymbol{A}-\boldsymbol{C}$, Learning curves from first $(\boldsymbol{A})$ and second $(\boldsymbol{B}, \boldsymbol{C})$ training phases. During the first training phase participants were trained for 15 sessions, so that they reached asymptotic learning $(\boldsymbol{A}$, black line). In session 1 there was additionally a pre-test on one position $(\boldsymbol{A}$, red dot). After completion of the first training phase participants started training on four positions for another 15 sessions: pre-tested (B, red line), exposed/identical offset ( $\boldsymbol{C}$, green line) exposed/random offset ( $\boldsymbol{C}$, blue line), and unstimulated ( $\boldsymbol{B} \& \boldsymbol{C}$, gray line). Colored areas represent SEM. $\boldsymbol{D}-\boldsymbol{F}$, Mean threshold levels for different learning stages. $\boldsymbol{D}$, Pre-testing results in better orientation discrimination performance in session 16. $\boldsymbol{E}$, No difference is seen between passive exposure and no stimulation during session 16. $\boldsymbol{F}$, Passive exposure conditions yield lower discrimination thresholds than pre-test or unstimulated for sessions 1719. Asterisks indicate $p<.05$. 
tested vs. exposed/random offset: $t_{(7)}=-0.842, p>.999$; and exposed/identical offset vs. exposed/random offset: $t_{(7)}=-1.972, p=.535$ ). For stage two (Figure 3F), a repeated measures ANOVA with exposure condition and session (17-19) as withinsubject factors revealed significant main effects of preconditioning type $(\mathrm{F}(3,21)=$ 9.559; $\mathrm{p}<.001)$ and session $(\mathrm{F}(2,14)=11.275 ; \mathrm{p}=.001)$ but a non-significant interaction $(\mathrm{F}(6,42)=1.218 ; \mathrm{p}=.317)$. Pairwise comparisons showed that the conditions in which masked exposure had been applied in the first training phase differed significantly from the pre-tested and no-stimulation conditions (exposed/random offset vs. unstimulated: $\mathrm{t}(7)=-3.795, \mathrm{p}=.041$; exposed/random offset vs. pre-tested: $\mathrm{t}(7)=-3.700, \mathrm{p}=.046$; exposed/identical offset vs. unstimulated: $\mathrm{t}(7)=-3.892, \mathrm{p}=.036$; exposed/identical offset vs. pre-tested: $\mathrm{t}(7)=$ $4.455, \mathrm{p}=.018)$. The difference between the two passive exposure conditions was not significant (random vs identical: $\mathrm{t}(7)=0.403, \mathrm{p}>.999$ ) as was the difference between unstimulated and pre-tested $\left(t_{(7)}=-0.409, p=1.000\right)$. The session effect was due to a higher threshold for session 17 compared to session 18 and 19 (session 17 vs. $18: t_{(7)}=4.145, p=.013$; session 17 vs. $19 t_{(7)}=4.050, p=.015$; session 18 vs. 19: $\left.t_{(7)}=1.373, p=.636\right)$. So, for stage two there were lower thresholds at the position where there had been masked exposure to stimuli during the first training phase, no matter whether the direction of the orientation offset had been identical or random with regard to stimuli shown during baseline training in the trained position during the first training phase. During the second stage of the second training phase, pre-tested and unstimulated positions were significantly worse than the two masked exposure conditions (Figure 3F). Notably, the advantage a pre-test gave over the unstimulated condition in the first session of the second training phase thus was already lost by the second session. In contrast, masked exposure led to a slightly more robust advantage in the second learning phase (and hence slightly more robust generalization), but this advantage became only visible in sessions 2-4 (stage 2) of the second training phase (i.e. sessions 17-19 in the total experiment, Figure 3F).

For stage three (see Figures 3B and C), a repeated measures ANOVA with preconditioning factor and session (20-30) revealed a non-significant main effects of preconditioning $\left(F_{(3,21)}=1.116 ; p=.365\right)$, a non-significant main effect of session $\left(F_{(10,70)}=0.838 ; p=.594\right)$ and no interaction $\left(F_{(4,36)}=0.912 ; p=.602\right)$. Thus, from 
session 5 onwards there were no significant differences between different exposure conditions.

\section{Mean percentage improvement}

To compare the improvement due to the different exposure conditions with that of baseline learning during the first training phase, we calculated mean percentage improvement for all 5 learning conditions (baseline learning: 55\%, pre-tested: 25\%, exposed/identical offset: $28 \%$, exposed/random offset: $32 \%$, unstimulated: $38 \%$ ). A repeated measure ANOVA comparing all five learning conditions (baseline learning, pre-tested, exposed/ random offset, exposed/identical offset, and unstimulated) showed that there was a significant main effect $\left(F_{(4,28)}=5.628 ; p=.002\right)$. Pairwise comparisons showed that only exposed/identical offset and baseline learning differed significantly in their percentage improvement $\left(t_{(7)}=-4.197, p=.041\right)$, while all other learning curves did not differ significantly from baseline learning (pretested vs. baseline learning: $t_{(7)}=-2.775, p=.275$; exposed/random offset vs. baseline learning: $t_{(7)}=-3.754, p=.071$; unstimulated vs. baseline learning: $t_{(7)}=-$ $3.468, p=.104)$. Comparisons of improvement indices among the learning curves in unstimulated, pre-tested, and masked exposure conditions were all far from significant (all paired-samples t-tests: $p$ >.999). This indicates that in the long run, none of the preconditioning strategies conferred any advantage for skill learning.

\section{Asymptotic performance of first and second training}

In Figure 3, asymptotic performance seems to be lower in the first than the second training phase. We therefore looked into asymptotic performance (mean over last nine session) of the exposure conditions and initial learning during the first training phase. A repeated measure ANOVA with condition (initial learning, pre-tested, exposed/random offset, exposed/identical offset, and unstimulated) as within-subject factor revealed no significant effect $\left(F_{(1.609,11.262)}=2.430 ; p=.139\right)$.

\section{Generalization indices}

To determine the transfer of baseline learning, generalization indices (see Methods, Data and statistical analyses) were calculated for the four preconditioning locations. The index used expressed the decrease of a threshold of interest of a second phase learning curve in a preconditioning location below the starting point of first phase 
baseline learning as a proportion of the improvement over the entire first phase baseline learning (for a similar specificity index see Ahissar \& Hochstein, 1997; Jeter, Dosher, Petrov, \& Lu, 2009). In this generalization index, 0\% indicates an absence of any transfer, and higher values indicate transfer. Computing the indices for the first threshold in the second training phase, Figure 4A shows no transfer to the no-stimulation location (index 12\%), but we found significant transfer to all three other preconditioned locations ( $41 \%$ on average) as established by three one sample t-tests testing the increase compared to $0 \%$ (all conditions: $p<.008$ ). This was also confirmed by a repeated measures ANOVA, which revealed a significant main effect of preconditioning $\left(F_{(3,21)}=4.699 ; p=.012\right)$. Hence, the generalization indices are a more sensitive tool to measure generalization from the first phase baseline learning location to the second-phase training in the preconditioned locations: Here some level of generalization is detected in the first session, for the two masked exposure locations as well as the pretested location. However, of the three stimulated pre-conditioning locations only the pre-tested location was significantly different from the unstimulated position (pre-tested vs. unstimulated

A

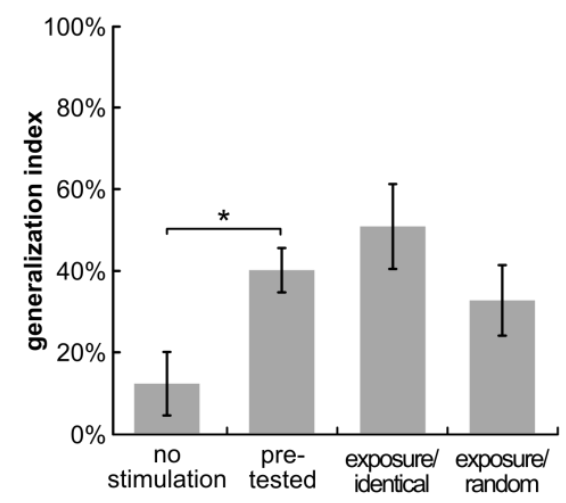

B

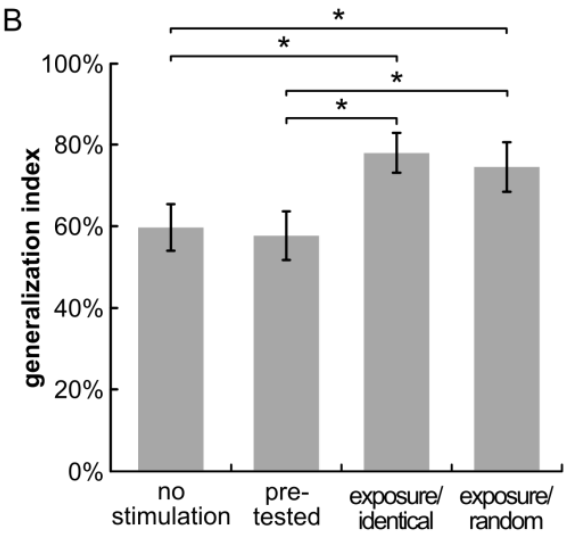

Figure 4. Generalization indices for the four pre-conditioning locations for session $16(\boldsymbol{A})$ and session 17-19 $(\boldsymbol{B})$. Stimulated conditions (pre-tested, exposed/identical and exposed/random) showed significant degrees of generalization in session 16. For session 17-19 generalization indices for passively exposed conditions showed more transfer than pre-tested or unstimulated positions. 
$t_{(7)}=-3.472, p=.031$; exposed/identical offset vs. unstimulated: $t_{(7)}=-2.787, p=$ .081 ; exposed/random offset vs. unstimulated: $\left.t_{(7)}=-1.693, p=.403\right)$.

We then calculated the generalization index also for the second stage (i.e., session 17-19). Note that these indices here are used to determine whether there was a maintained advantage of a specific type of preconditioning; the indices do not reflect a pure transfer from the baseline training location to the preconditioned locations. Figure 4B confirms a maintained advantage from preconditioning by masked exposure in sessions 17-19. Again, all exposure conditions showed significant degrees of generalization as measured by one sample t-tests (all conditions: $p<.001)$. A repeated measures ANOVA showed a significant main effect of exposure condition $\left(F_{(3,21)}=9.356 ; p<.001\right)$, and pairwise comparisons showed that the masked exposure conditions differed significantly from the pretested and unstimulated conditions (exposed/identical offset vs. pre-tested: $t_{(7)}=$ 3.985, $p=.032$; exposed/random offset vs. pre-tested: $t_{(7)}=-4.275, p=.022$; exposed/identical offset vs. unstimulated: $t_{(7)}=-3.875, p=.037$; exposed $/ \mathrm{random}$ offset vs. unstimulated: $\left.t_{(7)}=-3.772, p=.042\right)$. There was no significant difference between masked exposure conditions $\left(t_{(7)}=0.721, p>.999\right)$ or pre-tested and unstimulated conditions $\left(t_{(7)}=0.342, p>.999\right)$. Given the complete lack of differences among asymptotes in stage three of the second learning phase, we did not apply these specificity indices to test for maintained advantages of particular kinds of preconditioning.

\section{Control session}

After completion of the second training phase, participants underwent a control session during which they did the orientation task on five intermediate positions relative to the original positions used during the main experiment. The purpose of this control session was to test whether extended training on five positions would lead to complete generalization of orientation discrimination across the entire visual field, and whether the lack of difference between exposure conditions in stage three might be an artifact thereof. We therefore let participants perform three staircases on each intermediate control position and compared the resulting thresholds with the ones obtained from the original five positions (for details see Training Protocol). The results in Figure 4C show, however, that there was a consistent difference 
between the thresholds in the four pre-conditioning locations used in second-phase training, and the intermediate positions. A repeated measures ANOVA with preconditioning (pre-tested, exposed/identical, exposed/random, unstimulated) and stimulus position (original vs. intermediate) as within-subjects factor showed a significant main effect of position $\left(F_{(1,7)}=22.149 ; p=.002\right)$, but a non-significant main effect of pre-conditioning $\left(F_{(3,21)}=\right.$

$0.764 ; p=.527)$ and a non-significant interaction $\left(F_{(3,21)}=1.470 ; p=.251\right)$. Pairwise comparisons confirmed that performance at the four original positions was better than at the four control positions $\left(t_{(7)}=-5.967, p=.002\right)$ and thus performance improvements did not transfer completely (Figure 4C). A repeated measures ANOVA with all five locations (i.e., including also the position at which training during the first learning phase had taken place) yielded similar results. The data indicate that the lack of potential maintained advantages of specific kinds of preconditioning was not due to a full spread (and mixing) of expertise from the different trained positions.

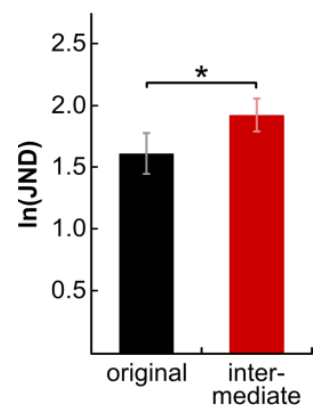

Figure 5. Mean performance for original and intermediate stimulus positions. The four original positions (unstimulated, pre-tested, masked-exposure/identical offset direction, masked exposure/random offset direction) were used during the main experiment over the course of 30 sessions, while the 5 intermediate positions were only used during the control session. 


\section{Discussion}

This study investigated to what extent different preconditioning strategies lead to spatial transfer of learning from a previously trained location, and how long-lasting these potentially advantageous effects of preconditioning are. We found that during initial generalization testing, there was only an advantage of the pre-tested condition over the unstimulated condition. However, for the second to fourth session, masked exposure yielded lower thresholds than either pre-tested or no-stimulation positions, irrespective of whether passively exposed stimuli had random or identical orientation offset to the stimulus used in the simultaneously performed task. From the fifth session onwards there was no difference between the four different exposure conditions. The type of preconditioning also made no difference regarding the mean percentage improvement over the course of the second 15-session training phase. The control experiment revealed that performance at intermediate control positions was significantly worse than that at positions used during the experiment. Hence, the extensive training at five different positions did not result in complete transfer, so that the absence of a maintained advantage of one or more types of preconditioning could not be explained by a full spread of expertise from the different trained positions.

In comparison to the findings of T. Zhang et al. (2010), where they showed that a brief pre-test could result in complete transfer to another retinal position, we found that there was a lack of complete transfer in the first session of the second training phase. The number of trials we used for the pre-test was very similar to what $\mathrm{T}$. Zhang et al (2010) did (we had 240 trials in 4 staircases, compared to 200 trials in 6 staircases). As it has been shown that prolonged training at threshold promotes location specificity (Hung \& Seitz, 2014), the lack of complete generalization in our experiment is likely due to the extensive training during the first training phase resulting in asymptotic learning.

In our study, extended exposure to masked, unattended stimuli gave a slight advantage over pre-tested and no-stimulation positions for early learning (session 24). Previous studies only looked at transfer immediately after exposure and did not explore how learning develops further. J.-Y. Zhang and colleagues (2010) applied a training-plus-exposure procedure to test for orientation specificity in orientation and 
contrast training. This procedure was different from our masked exposure conditions, since participants performed a task at the transfer location on an irrelevant feature (e.g. contrast) while that stimulus still contained the (future) relevant feature (e.g. orientation). There were no control experiments in J.-Y. Zhang et al. (2010) that assessed the visibility/awareness of or attention to the stimulus feature during this form of exposure, and hence it is difficult to interpret any transfer to this exposure location of expertise on that feature after training in another location. The passive exposure experiment of Wang et al. (2012) is somewhat closer to our exposure conditions. They used passive exposure to see how an irrelevant and non-demanding stimulus (compared to more relevant and demanding conditions) influenced transfer in discrimination learning. In their experiment, participants performed a Vernier discrimination task in one location while being passively exposed to an orthogonal Vernier stimulus in another visual field quadrant. Their passive stimuli were unmasked and presented for $200 \mathrm{~ms}$, just as the stimulus in the trained quadrant. However, this again raises the concern that the passive stimuli in fact could have been attended. Our stimuli by contrast were presented for $33 \mathrm{~ms}$ and followed by a $49 \mathrm{~ms}$ mask and after finishing the first training phase a visibility rating was done, which confirmed that all but one participant were unaware of the masked Gabor. Wang et al. (2012) reported no transfer to the exposed position. In our study, when considering the first threshold of the second training phase, we found significant transfer from the baseline-trained position to the pre-conditioned locations as supported by generalization indices.

It has been argued before that passive (i.e. unattended) exposure in some conditions can lead to a form of perceptual learning. In an interesting study, Gutnisky and colleagues (2009) investigated the effects of exposure-based learning. During an exposure phase, which lasted three minutes, gratings (alternating between two orientation) were flashed $(200 \mathrm{~ms}$ on, $200 \mathrm{~ms}$ off) at three different locations of which one was attended, another one unattended, and the third functioned as control condition (exposure to random orientations). At the attended location participants performed a contrast detection task. Over the course of ten daily sessions the authors looked at performance changes in the orientation discrimination task which was always preceded by the exposure phase. Orientation discrimination performance was 
best in the attended exposure location, intermediate in the unattended exposure location and worst in the control location. Interestingly, the effects of unattended exposure generalized over orientations, whereas beneficial effects of attended exposure were restricted to the orientation used during exposure. Although our finding of a slight advantage for learning after unattended exposure is in line with the findings of Gutnisky et al. (2009), there are some methodological differences between our studies. Exposure and training were interleaved in Gutnisky et al. 's (2009) study, whereas we had a strictly sequential design with 15 sessions of masked exposure before testing generalization from prior training to these preconditioned locations over the course of 15 sessions. In the study by Gutnisky et al. (2009), effects of exposure and learning were intermingled since both happened every day, which means that only by relative differences between conditions the exposure effect can be revealed. In our study, pure effects of exposure were demonstrated but we also showed that the advantages afforded by exposure were temporary and disappeared with continued training. Gutnisky and colleagues (2009), controlled attentional allocation during exposure by having participants perform an irrelevant task at the attended exposure position. In contrast both of our passive exposure conditions were fully unattended (and stimuli were very brief and additionally masked), while on a different position the relevant task was performed. Given these methodological differences, awareness of exposed stimuli is to be expected for their study but not ours. Thus, the differences between our findings (i.e., very limited generalization to exposed positions) and theirs (i.e., generalization up to $30^{\circ}$ orientation difference) regarding exposure to unattended stimuli, are likely explained by differences in awareness of the exposure stimuli and by the interleaving of exposure and learning in Gutnisky et al. (2009) as opposed to the sequential administration of exposure and learning in our study.

Our masked exposure paradigm to some extent can be compared with the subliminal learning studies from Watanabe and Seitz (Seitz \& Watanabe, 2003; Watanabe et al., 2002; Watanabe et al., 2001). In their studies, it is shown that perceptual learning happens when stimuli are not perceived (Watanabe et al., 2001). In further studies on task-irrelevant perceptual learning it has been shown that parathreshold but not suprathreshold stimuli lead to a form of learning (Tsushima et 
al., 2008). This result was interpreted as showing that weak stimuli pass the attentional filter unnoticed and therefore fail to be suppressed by the attentional system, whereas strong stimuli are actively inhibited. The short stimulus duration and masking of the passively presented stimuli in our study might thus have prevented inhibition by the attentional system and allowed partial transfer of learning to the exposed positions. One mechanism enabling task-irrelevant learning is thought to be stimulus-reward pairing, whereby stimulus driven signals from the irrelevant stimulus and task driven signals, including reinforcement signals must coincide (Seitz, Kim, \& Watanabe, 2009; Seitz \& Watanabe, 2005). One might argue that the in the present study feedback on task performance functions as reinforcement signal; however it seems unlikely that feedback has the same effect as actual reward. Our data suggest that in the present paradigm the basis of the observed limited generalization must be some kind of adaptation, since no difference between passive exposure conditions with either identical or random orientation offsets was observed.

Our data confirm that a brief pre-test as well as masked exposure result in some degree of generalization. Although pre-testing in theory should favor generalization, the observed pre-testing effect was small and of limited duration when training was continued. No advantage was seen for asymptotic learning. In light of these findings, the claim of T. Zhang et al. (2010) that a brief pre-test leads enables complete transfer seems unwarranted. As has been shown by Hung and Seitz (2014) the amount of training at threshold determines whether transfer of specificity is observed. Given the more extensive training at asymptotic performance levels in our study it is thus logical that our generalization effect is marginal. The generalization effect after pre-test was only seen during early learning, and did not extend to asymptotic learning. Similarly, advantages after masked exposure only speeded up early learning and were not long-lasting. The observed masked-exposure effect is generally in line with existing literature showing that unattended stimuli may yield some learning. The lack of a difference between exposure to stimuli with identical and random orientation off-set as the stimuli used during simultaneous active learning suggests that some kind of adaptation is causing this effect. 
Previous studies investigating effects of pre-test or exposure on learning have been using a single session to assess generalization, which precludes assessment of the robustness of these advantages of these forms of preconditioning (Wang et al., 2012; J.-Y. Zhang et al., 2010; T. Zhang et al., 2010). In addition, most studies used brief learning curves to induce generalization. The true implications of effects of preconditioning can only be assessed if (1) the initial training is long enough and (2) if the effect of preconditioning in other locations is tested for more than a single day, and ideally for an entire learning curve. To our knowledge we are the first to comply with these requirements and we find that that although there are some weak advantages of the different forms of preconditioning assessed, these are temporary effects limited to early phases of learning curves and irrelevant for asymptotic learning. This indicates that despite a growing body of literature showing forms of learning that can take place without attention, attention is still the single most important factor driving (asymptotic) perceptual learning. 


\section{Acknowledgements}

GL was supported by a grant from the Netherlands Organization for Scientific Research to the FPN Graduate School for Cognitive and Clinical Neuroscience (NWO, 022.001.036). PDW was supported by a grant from the Netherlands Organization for Scientific Research (NWO VICI grant, 453.04.002). 


\section{References}

Ahissar, M., \& Hochstein, S. (1997). Task difficulty and the specificity of perceptual learning. Nature, 387(6631), 401-406.

Bruchmann, M., Breitmeyer, B. G., \& Pantev, C. (2010). Metacontrast masking within and between visual channels: Effects of orientation and spatial frequency contrasts. Journal of Vision, 10(6), 12.

De Weerd, P., Pinaud, R., \& Bertini, G. (2006). Plasticity in v1 induced by perceptual learning. New York: Springer Science + Business Media.

Fahle, M. (1997). Specificity of learning curvature, orientation, and vernier discriminations. Vision Research, 37(14), 1885-1895.

Grill-Spector, K., Henson, R., \& Martin, A. (2006). Repetition and the brain: Neural models of stimulus-specific effects. Trends in Cognitive Sciences, 10(1), 14-23.

Gutnisky, D. A., Hansen, B. J., Iliescu, B. F., \& Dragoi, V. (2009). Attention alters visual plasticity during exposure-based learning. Current Biology, 19(7), 555-560.

Harris, H., Gliksberg, M., \& Sagi, D. (2012). Generalized perceptual learning in the absence of sensory adaptation. Current Biology, 22(19), 1813-1817.

Hung, S.-C., \& Seitz, A. R. (2014). Prolonged training at threshold promotes robust retinotopic specificity in perceptual learning. The Journal of Neuroscience, 34(25), 8423-8431.

Jeter, P. E., Dosher, B. A., Petrov, A., \& Lu, Z.-L. (2009). Task precision at transfer determines specificity of perceptual learning. Journal of Vision, 9(3), 1-13.

Karni, A. (1996). The acquisition of perceptual and motor skills: A memory system in the adult human cortex. Cognitive Brain Research, 5(1-2), 39-48.

Karni, A., \& Bertini, G. (1997). Learning perceptual skills: Behavioral probes into adult cortical plasticity. Current Opinion in Neurobiology, 7(4), 530-535.

Karni, A., Meyer, G., Jezzard, P., Adams, M. M., Turner, R., \& Ungerleider, L. G. (1995). Functional mri evidence for adult motor cortex plasticity during motor skill learning: Nature Publishing Group.

Karni, A., Meyer, G., Rey-Hipolito, C., Jezzard, P., Adams, M. M., Turner, R., \& Ungerleider, L. G. (1998). The acquisition of skilled motor performance: Fast and slow experience-driven changes in primary motor cortex. Proceedings of the National Academy of Sciences, 95(3), 861-868. 
Karni, A., \& Sagi, D. (1991). Where practice makes perfect in texture discrimination: Evidence for primary visual cortex plasticity. Proceedings of the National Academy of Sciences, 88(11), 4966-4970.

Karni, A., \& Sagi, D. (1993). The time course of learning a visual skill. Nature, 365(6443), 250-252.

Macknik, S. L., \& Livingstone, M. S. (1998). Neuronal correlates of visibility and invisibility in the primate visual system. Nature Neuroscience, 1(2), 144-149.

Regan, D., \& Beverley, K. (1985). Postadaptation orientation discrimination. Journal of the Optical Society of America A, 2(2), 147-155.

Schoups, A. A., Vogels, R., \& Orban, G. A. (1995). Human perceptual learning in identifying the oblique orientation: Retinotopy, orientation specificity and monocularity. Journal of Physiology, 483(Pt 3), 797-810.

Schwartz, S., Maquet, P., \& Frith, C. (2002). Neural correlates of perceptual learning: A functional mri study of visual texture discrimination. Proceedings of the National Academy of Sciences, 99(26), 17137-17142.

Seitz, A. R., Kim, D., \& Watanabe, T. (2009). Rewards evoke learning of unconsciously processed visual stimuli in adult humans. Neuron, 61(5), 700-707.

Seitz, A. R., \& Watanabe, T. (2003). Psychophysics: Is subliminal learning really passive? Nature, 422(6927), 36-36.

Seitz, A. R., \& Watanabe, T. (2005). A unified model for perceptual learning. Trends in Cognitive Sciences, 9(7), 329-334.

Tsushima, Y., Seitz, A. R., \& Watanabe, T. (2008). Task-irrelevant learning occurs only when the irrelevant feature is weak. Current Biology, 18(12), R516-R517.

Wang, R., Zhang, J.-Y., Klein, S. A., Levi, D. M., \& Yu, C. (2012). Task relevancy and demand modulate double-training enabled transfer of perceptual learning. Vision Research, 61(0), 33-38.

Watanabe, T., Nanez, J. E., Koyama, S., Mukai, I., Liederman, J., \& Sasaki, Y. (2002). Greater plasticity in lower-level than higher-level visual motion processing in a passive perceptual learning task. Nature Neuroscience, 5(10), 1003-1009.

Watanabe, T., Náñez, J. E., \& Sasaki, Y. (2001). Perceptual learning without perception. Nature, 413(6858), 844-848.

Wetherill, G. B., \& Levitt, H. (1965). Sequential estimation of points on a psychometric function. British Journal of Mathematical and Statistical Psychology, $18(1), 1-10$. 
Xiao, L.-Q., Zhang, J.-Y., Wang, R., Klein, S. A., Levi, D. M., \& Yu, C. (2008).

Complete transfer of perceptual learning across retinal locations enabled by double training. Current Biology, 18(24), 1922-1926.

Zhang, J.-Y., Zhang, G.-L., Xiao, L.-Q., Klein, S. A., Levi, D. M., \& Yu, C. (2010). Rule-based learning explains visual perceptual learning and its specificity and transfer. The Journal of Neuroscience, 30(37), 12323-12328.

Zhang, T., Xiao, L.-Q., Klein, S. A., Levi, D. M., \& Yu, C. (2010). Decoupling location specificity from perceptual learning of orientation discrimination. Vision Research, 50(4), 368-374. 


\title{
Chapter 4
}

\author{
Long-term successive training causes
}

behavioral interference

Lange, G.*, Senden, M.*, Radermacher, A., Goebel, R., \& De Weerd, P. (in preparation). Long-term successive training causes behavioral interference.

*shared first authorship 


\section{Abstract}

Previous studies have shown that skill learning, although quite stable in general, can be interfered with by additional training at a later time on similar tasks/stimuli. Commonly, these behavioral interference effects are attributed to the disruption of a time-limited offline memory consolidation process. Interestingly, behavioral interference has been shown to occur for time intervals of up to 24 hours (Been, Jans, \& De Weerd, 2011) or longer (Caithness et al., 2004). This raises the question whether it is best accounted for by disrupted consolidation. Alternatively, memory traces after their formation may remain malleable, and behavioral interference might occur when memory formation in two tasks recruits a largely shared (i.e. insufficiently segregated) neuronal population subjected to incompatible requirements on network connectivity. To evaluate this possibility, we first implemented a learning mechanism in a recurrent model of orientation selectivity characterized by representational overlap. We used this neural network model to simulate the effects of orientation discrimination learning and to simulate the effects of varying contributions of consolidation on the interference between two tasks. In these tasks, efficient orientation discrimination learning required opposite network connectivity changes. Secondly, we designed an experiment, in which training in the two tasks was done serially in time blocks of three weeks, thereby maximizing the possibility that one task would induce a fully consolidated memory trace before onset of training the other one. Nevertheless, we found strong interference between the two tasks, similar to what has been observed for intervals in the range of $0-24 \mathrm{~h}$ (Been et al., 2011). Our model simulations show that our results can be more straightforwardly explained in terms of reactivation and representational competition than in terms of disruption of consolidation. 


\section{Introduction}

Extensive skill training results in performance levels that can remain stable for several years (Karni, 1996; Karni \& Sagi, 1993). Skill acquisition is, however, vulnerable to interference. Behavioral studies have shown that following training in a first task, training in a similar second task results in interference if the time interval is limited to a few hours (Seitz et al., 2005), but not if the time interval exceeds $\sim 4 \mathrm{~h}$ (Brashers-Krug, Shadmehr, \& Bizzi, 1996; R Shadmehr \& Holcomb, 1997; Zhang et al., 2008). This time-dependency suggests that behavioral interference disrupts a time-limited consolidation process. Consolidation is often considered to last up to 46 hours, in line with the time window in which the first cascade of plasticity-related early and late gene expression occurs following stimulation (Casadio et al., 1999; Reymann \& Frey, 2007). However, reports of behavioral interference after delays ranging from 24 hours up to a week (Been et al., 2011; Caithness et al., 2004; Goedert \& Willingham, 2002; Reza Shadmehr \& Brashers-Krug, 1997) call this interpretation into question.

To explain interference over long time intervals, one possibility is that consolidation takes a long time. The idea of protracted consolidation is supported by evidence for multiple periods of consolidation during both wakefulness (Bourtchouladze et al., 1998; Korman, Raz, Flash, \& Karni, 2003; Wanisch, Wotjak, \& Engelmann, 2008) and sleep (Karni, Tanne, Barton, Askenasy, \& Sagi, 1994; Stickgold, James, \& Hobson, 2000). Moreover, in the domain of episodic memory (Squire \& Zola-Morgan, 1991), it has been suggested that consolidation takes many years. However, both for episodic memory (Forcato et al., 2007; Hupbach, Gomez, \& Nadel, 2009) and skill learning (Walker, Brakefield, Hobson, \& Stickgold, 2003), new theoretical views have been formulated in which memories do not consolidate, but rather exist in a dormant state, or in a reactivated state during which the memory trace can be modified. Here, we aim to weigh these two views against each other in the context of asymptotic skill learning in a visual orientation discrimination task. Asymptotic visual skill learning involves low-level sensory areas such as V1 (Ahissar \& Hochstein, 2004; Hochstein \& Ahissar, 2002; Karni \& Bertini, 1997; Willingham, Wells, Farrell, \& Stemwedel, 2000) whose neuronal populations have been shown to sharpen their tuning curves (Schoups, Vogels, Qian, \& Orban, 2001; 
see also Raiguel, Vogels, Mysore, \& Orban, 2006). According to simulation studies, these tuning changes could be part of a population response to training in which the preferred orientation of stimulated neurons shifts somewhat in the direction of the trained reference orientation (Teich \& Qian, 2003). Neurons recruited by the training thus show sharper tuning curves combined with greater density (peaks of tuning curves close together), whereas neurons not recruited by the training tend to show the opposite trends. These training-induced population effects can be expected to shift with the trained reference orientation, such that the demands on population changes and associated network connectivity changes may become incompatible for a range of orientation differences between reference orientations. In these cases, unless neuronal populations in V1 possess suitable redundancy permitting separate subpopulations to change their tuning properties to suit training at different reference orientations, training at one reference orientation can be expected to always interfere with training at the other reference orientation, irrespective of the time interval between the training sessions devoted to the two reference orientations. Thus, if two tasks recruit different but largely overlapping populations, consolidation could be adaptive if the overlapping neuronal populations were indeed separable during memory formation, whereas in the absence of separability, it is more adaptive for memory traces to remain modifiable (see Discussion).

The contributions of overlapping populations and consolidation processes to behavioral interference effects on asymptotic learning have recently been investigated by Been et al. (2011). The investigators initially trained participants on an oblique reference stimulus (T1) and subsequently provided additional training (T2) on either similar or dissimilar reference orientations, respectively differing by $\pm 30^{\circ}$ and $90^{\circ}$ from oblique. In line with an overlapping population hypothesis, the authors observed behavioral interference for similar but not for dissimilar reference orientations. This effect was tested further by varying the T1-T2 interval in a range from zero to 24 hours. Behavioral interference on asymptotic learning was present for all tested time-intervals. Hence, interference was determined by population overlap and not by the time interval between tasks.

Although these data make a consolidation mechanism operating in the range of a number of hours an unlikely contributor to memory formation in our task, the lack 
of a decline in interference for time intervals increasing up to $24 \mathrm{~h}$ can still be in line with a consolidation process lasting $>24 \mathrm{~h}$. In Been et al. (2011), the fast switching between T1 and T2 might have prevented consolidation and segregation of the neuronal population into subpopulations each carrying a separate memory trace. Here, we tested this idea in a new experimental design in which a task was trained for three weeks before switching to training for three weeks in the next task. To test possible consequences of this experiment on interference under a consolidation hypothesis and under an overlapping populations hypothesis, we implemented the recurrent model of V1 orientation selectivity proposed by Teich and Qian (2003, 2006, 2010) and extended it to learn on-line in response to (changing) task demands. The resulting model was able to predict perceptual learning of orientation discrimination and interference effects in Been et al. (2011). The model additionally predicted an interference effect in the present experiment using three-week training blocks, which was confirmed by empirical data. Combined, our psychophysical and simulation data suggest that in the task conditions used, interference results from incompatible, competing demands on network connectivity when two tasks recruit largely the same neural population (competition hypothesis), rather than from timelimited plasticity (consolidation hypothesis). 


\section{Methods}

\section{Participants}

Six participants (mean age 25.32, $S D 3.45,4$ female), naïve to the purpose of the study, participated in the main experiment and the control conditions. All participants had normal or corrected-to normal visual acuity. Informed, written and verbal consent was obtained according to the Helsinki Declaration, after full information about all procedures and the right to withdraw participation at any time. All procedures were approved by the local Ethical Committee of the Faculty of Psychology and Neuroscience (ECP). For their participation in the study participants received either monetary reward or credits to fulfill course requirements.

\section{Stimuli, task and procedure}

Gabor patches oriented at $135^{\circ}, 105^{\circ}$, or $165^{\circ}$ were used as stimuli $(2.37$ cycles/degree spatial frequency, 50\% Michelson contrast, $3^{\circ}$ diameter). The Gabor stimulus was presented at $6^{\circ}$ eccentricity, and its average luminance was $56 \mathrm{~cd} / \mathrm{m}^{2}$, which was equal to the luminance of the background. The stimuli were presented in all four quadrants of the visual field. Participants were placed in a dimly lit room; their head was supported by a chin and head rest keeping eye-screen distance constant at $57 \mathrm{~cm}$. Visual stimuli were displayed on a 19" Samsung SyncMaster 940BF LCD monitor (Samsung, Seoul, South Korea; 60Hz refresh rate, 1280x1024 resolution). The screen was covered by a gray mask with an oval aperture so that the screen borders were not visible to participants and thus could not be used as reference for the orientation discrimination task. Fixation control was monitored with a Viewpoint Eyetracker v.2.8.3 (Arrington Research, Inc., Scottsdale, Arizona, USA; $60 \mathrm{~Hz}$ sampling rate, $37 \mathrm{pixel} / \mathrm{degree}$ spatial resolution). Stimulus presentation and response recording was performed by Cortex v.5.9.6 (NIH freeware for psychophysical and neurophysiological experimentation).

Participants performed an orientation discrimination task with an unseen oblique orientation as reference stimulus. They indicated the direction of the orientation offset by pressing either the right or left arrow key for clockwise and counterclockwise rotations respectively (Figure 1). Each experimental trial started with a time window of maximally $750 \mathrm{~ms}$ during which accurate fixation was to be 
initiated (i.e., deviation $<1.5^{\circ}$ from fixation dot). Once initiated, fixation had to be maintained for another $250 \mathrm{~ms}$ to trigger stimulus presentation, and furthermore had to be maintained throughout presentation of the Gabor stimulus $(500 \mathrm{~ms})$ in one of the four quadrants of the visual field. Stimulus presentation was then followed by a $1000 \mathrm{~ms}$ response window. Responses were given with the right middle and index finger, to indicate clockwise or counterclockwise deviation from the reference orientation, respectively. After each trial, feedback was given to participants by changing the color of the fixation dot for $200 \mathrm{~ms}$ to either green or red for correct and incorrect responses, respectively. After feedback, the fixation dot disappeared signifying the end of the trial, which was then followed by a fixed 500ms inter-trial interval. When a participant's gaze fell outside the fixation window during the fixation period, trials were aborted. Aborted trials were repeated at a randomly chosen time during the experiment.

In the present study, we conducted three experiments. One experiment was designed to test whether a 3 -week training at the $135^{\circ}$ reference orientation would also be interfered with by subsequent 3 -week training at reference orientations $105^{\circ}$ and $165^{\circ}$ (backward interference). Another experiment was designed to test whether a 3- week training at reference orientations $105^{\circ}$ and $165^{\circ}$ in a single location would have an interfering effect on subsequent training in the same location at the $135^{\circ}$ reference orientation (forward interference). A control experiment was designed to test whether a 3 -week training at the $135^{\circ}$ reference orientation would also be interfered with by a subsequent 3 -week training at a $45^{\circ}$ reference. The sequences of
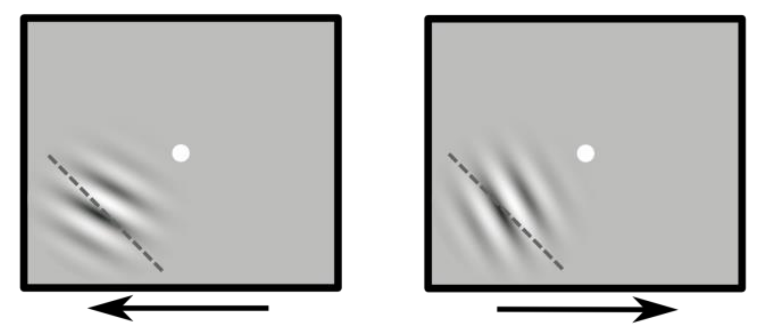

Figure 1. Orientation discrimination task and stimuli. Thresholds were determined using an identification design, with left key responses for counterclockwise orientation offsets from a $135^{\circ}$ reference (dashed line, not shown to participants), and right key responses for clockwise orientation offsets. Stimuli are enlarged for illustration. 
training at different reference orientations and the orientation differences among reference orientations in the different experiments were based on Been et al., (2011). However, in contrast to Been et al. (2011), we trained participants to reach asymptotic performance levels at the $135^{\circ}$ orientation before starting training at the potentially interfering reference orientations of $105^{\circ}$ and $165^{\circ}$ (or vice versa), to test whether these extended periods of undisturbed training would diminish interference between the two training periods. Note that we will refer to forward and backward interference experiments in accordance with the hypothesized interference effects in these two experiments.

Figure 2 shows how these three experiments were combined into a single study design. The study consisted of three Parts, each lasting for 15 sessions. During each session, participants completed the four conditions belonging to a Part, with each condition representing training at a specific reference orientation and in a specific visual field quadrant. For each condition (i.e. each frame in Figure 2), participants completed four staircases, so that they performed 16 staircase measurements per session. Thus, participants spent 15 such sessions performing Part A, then 15 sessions performing Part B, and then 15 sessions performing Part C.

After performing the 45 sessions, all three planned experiments were completed, and analysis would be based on three separate selections of conditions representing the three experiments (color-coded in red, blue and green in Figure 2). The backward interference experiment is indicated by blue frames in Figure 2: an initial 15 sessions of baseline training at a $135^{\circ}$ reference orientation (Part $A, E_{135}$ ) were followed by 15 sessions of training at reference orientations differing by $\pm 30^{\circ}$ from the $135^{\circ}$ reference orientation (Part $B, E_{105} \& E_{165}$ ). The extent of interference was then tested in another 15 sessions of training at the $135^{\circ}$ reference orientation (Part $C, E_{135}$ ) with all stimuli in the same location. The forward interference experiment was composed of conditions indicated by red frames in Figure 2: Training with reference orientations $105^{\circ}$ and $165^{\circ}$ (Part $\left.A, E_{165} \& E_{105}\right)$ preceded training at the $135^{\circ}$ reference orientation (Part $B, E_{135}$ ). Training data obtained at a $135^{\circ}$ reference orientation in a different quadrant (Part A, $E_{135}$, blue/red frame) was used as baseline. In a third, control experiment, indicated by green frames, initial baseline training at the reference orientation of $135^{\circ}$ (Part $\left.A, C_{135}\right)$ was followed by training 
at an orthogonal reference orientation (Part $\left.B, C_{45}\right)$ and subsequently training at the $135^{\circ}$ reference orientation again $\left(\right.$ Part $\left.C, C_{135}\right)$ to test for any potential interference effects from the intervening training at the orthogonal reference. This was a control experiment, as training at a reference orientation differing by $90^{\circ}$ from the original reference orientation has been shown previously not to interfere with original learning. Note that the training at $105^{\circ}$ and $165^{\circ}$ reference orientations in Part $\mathrm{C}$ had the sole purpose of keeping the structure of daily training sessions (and effects of fatigue) constant throughout the experiment.
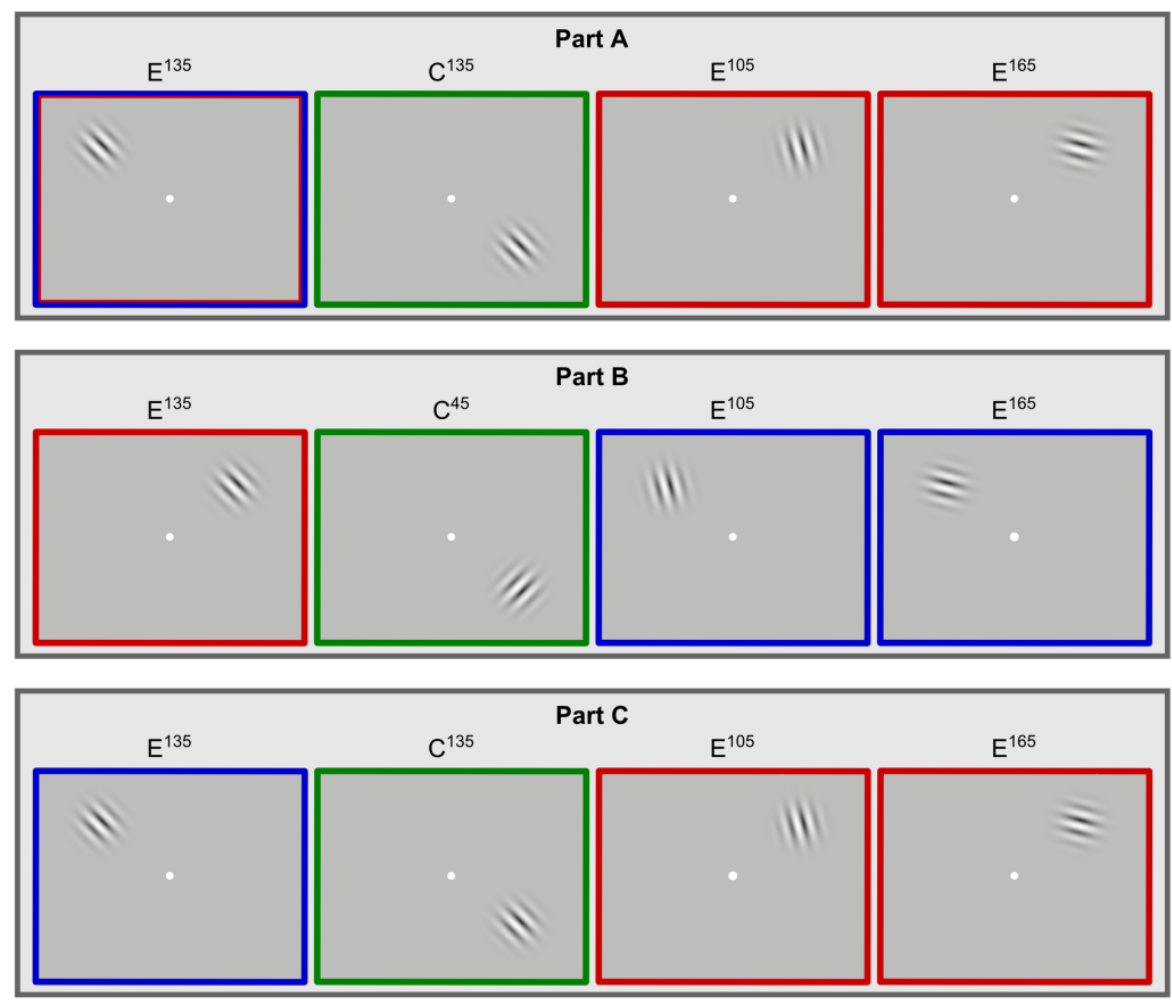

Figure 2. Design of the study. The study was split in three parts (A, B and C), each part consisting of four conditions. Parts A, B, and C lasted 15 sessions each and were done consecutively (order of conditions within each part was counterbalanced). In total, the study took 45 sessions. Conditions are labeled as control (C) or experimental (E) and followed by the reference orientation used. The frame color indicates which conditions belong together. 
In the execution of the study, condition order was counterbalanced across days/participants. Furthermore, the quadrants used for specific experimental conditions were counterbalanced across participants. Within participants, testing schedules were kept constant (i.e., at least three sessions per week, all at the same time of day) for the duration of the experiment. Importantly, although the testing blocks were completed in 15 daily sessions, the calender length for the full training in each block comprised 4-5 weeks, as there was no testing on weekends, and as participants typically could be tested only 3 to 4 times per week. The extended length of the three training periods due to pauses in training can be thought of as favoring consolidation (see Discussion).

\section{Threshold measurements}

We used a Wetherill \& Levitt (1965) staircase tracking 84\% correct performance to acquire just-noticeable differences (JNDs). The staircase ended when either 14 reversal points were acquired or 120 trials were completed. In total participants completed 16 staircases in each session, and a total of 720 staircases for each complete experiment. From each staircase, the last ten reversal points were selected to contribute to threshold estimation. Since four staircases were done per quadrant in each session, we determined the threshold per quadrant/session as the geometric mean of 40 reversal points. In the first session the start level of each staircase was set to an orientation deviation of approximately $15^{\circ}$, and for all subsequent sessions the average threshold level of the previous session was taken as starting level. Orientation deviation was adapted to performance in a proportional manner, by either dividing or multiplying the current orientation difference by a factor 1.2, depending on performance criteria designed for the staircase to converge on a $84 \%$ correct level. All thresholds were ln-transformed.

\section{Model Simulations}

\section{Model of Orientation Discrimination}

V1 orientation tuning and adaptation was simulated using a recurrent model first described by Teich and Qian (2003, 2006, 2010). Briefly, the model assumes that weakly orientation tuned feedforward information entering V1 from subcortical regions is sharpened by recurrent excitatory and inhibitory synapses among V1 
neurons. In accordance with the descriptions given in Teich and Qian (2003) the model consists of $N$ neurons with their preferred orientations $\theta$ covering 180 degrees. The firing rate $R(\theta, \Phi, t)$ of each neuron with preferred orientation $\theta$ and presented with stimulus orientation $\Phi$ at time $t$ is given by

$R=\alpha \max (V, 0)$

where $\alpha$ is a gain factor and $V$ is the membrane potential which obeys the equation

$\tau \frac{\Delta V}{\Delta t}=-V+I_{f}+I_{r}$

In equation 2), $\tau$ is the membrane time constant, $I_{f}$ the feed-forward input to the neuron, and $I_{r}$ the recurrent input a neuron receives from its neighbors. The feedforward input resulting from presenting stimulus orientation $\Phi$ to a neuron with preferred orientation $\theta$ is given by

$I_{f}(\theta, \Phi)=J_{f} \exp \left[-\frac{(\theta-\Phi)^{2}}{2 \sigma_{f}^{2}}\right]$

where $J_{f}$ and $\sigma_{f}$ respectively determine the strength and width of the input. The recurrent input neuron $i$ with preferred orientation $\theta_{i}$ receives is the total activation it receives from neurons $j$ with preferred orientations $\theta_{j}$

$I_{r}\left(\theta_{i}, t\right)=\sum_{-90^{\circ}}^{90^{\circ}} w_{i, j} R\left(\theta_{j}, t\right)$

where $w_{i, j}$ is the weight from neuron $j$ with preferred orientation $\theta_{j}$ to neuron $i$ with preferred orientation $\theta_{i}$. Before learning the weight between two neurons $i$ and $j$ with respective preferred orientations $\theta_{i}$ and $\theta_{j}$ is initialized as

$w_{i, j}=E\left(\theta_{i}-\theta_{j}\right)-I\left(\theta_{i}-\theta_{j}\right)$ 
with $E(\theta)$ and $I(\theta)$ being the excitatory and inhibitory connection probability distributions expressed by the periodic functions

$E(\theta)=c_{e}[\cos (2 \theta)+1]^{a_{e}}$

and

$I(\theta)=c_{i}[\cos (2 \theta)+1]^{a_{i}}$.

In equations 6 and 7, the exponents $a_{e}$ and $a_{i}$ control the sharpness of the excitatory and inhibitory distributions, respectively. The constants $c_{e}$ and $c_{i}$ are normalization factors ensuring that the sum of each distribution equals unity.

Behavioral Performance and Learning

In order to compare the performance of the model with that of our subjects, the distribution of firing rates across neurons was translated into a decision of whether the currently presented orientation was rotated clockwise or counterclockwise with respect to a reference orientation. This was done using signal detection theory (Green \& Swets, 1966). Specifically, the mean response of neuron $i$ with preferred orientation $\theta$ to a stimulus with orientation $\Phi$ is a function of the firing rate this stimulus evokes in the neuron $r_{i}(\Phi)$ and the duration of stimulation $\Delta t$

$m_{i}(\Phi)=r_{i}(\Phi) \Delta \mathrm{t}$

Furthermore, the variance of the firing rate under identical stimulus conditions was proportional to the mean response

$\operatorname{var}_{i}(\Phi)=k m_{i}(\Phi)$

where $k$ is a dimensionless constant between 1 and 4 (Peres \& Hochstein, 1994; Shadlen \& Newsome, 1994; Snowden, Treue, \& Andersen, 1992; Softky \& Koch, 1993). The activity $x_{i}\left(k m_{i}(\Phi)\right)$ neuron $i$ produces when presented with stimulus $\Phi$ 
can be estimated by sampling from the normal distribution $\mathrm{N}\left[m_{i}(\Phi), \sqrt{k m_{i}(\Phi)}\right]$. In order to make a decision whether a probe stimulus $\Phi_{\text {probe }}$ presented on a given trial was orientated clockwise or counterclockwise with respect to a reference stimulus $\Phi_{\text {ref }}$, each neuron calculated the difference of activity resulting from the two stimuli. This difference $\left(x_{i} \Phi_{\text {ref }}-x_{i} \Phi_{\text {probe }}\right)$ follows the normal distribution $\aleph\left(m \Phi_{\text {ref }}-m \Phi_{\text {probe }}, \sqrt{k\left(m_{i} \Phi_{\text {ref }}-m \Phi_{\text {probe }}\right)}\right.$. Neuron $i$ would make a correct decision either if it prefers $\Phi_{\text {ref }}$ over $\Phi_{\text {probe }}$ and if $x_{i} \Phi_{\text {ref }}-x_{i} \Phi_{\text {probe }}>0$, or if it prefers $\Phi_{\text {probe }}$ over $\Phi_{\text {ref }}$ and if $x_{i} \Phi_{\text {ref }}-x_{i} \Phi_{\text {probe }}<0$. Taking these two cases together the probability that neuron $i$ makes a correct decision (Green \& Swets, 1966; Lehky \& Sejnowski, 1990; Teich \& Qian, 2003) is given by

$p_{i}=\frac{1}{2} \operatorname{erfc}\left(\frac{d^{\prime}}{\sqrt{2}}\right)$

with

$d^{\prime}=\frac{\left|m_{i} \Phi_{\text {ref }}-m_{i} \Phi_{\text {probe }}\right|}{\sqrt{k\left[m_{i} \Phi_{\text {ref }}+m_{i} \Phi_{\text {probe }}\right]}}$

and $\operatorname{erfc}(\cdot)$ being the complementary error function. A majority vote determined the performance of the population on a given trial, with a decision deemed correct if at least 55 percent of all individual neurons make a correct decision.

Learning, in our model, was a direct consequence of population performance within trials as it was implemented in the form of an error-triggered anti-Hebbian learning rule (Adini, Sagi, \& Tsodyks, 2002; Koch, Ponzo, Di Lorenzo, Caltagirone, \& Veniero, 2013; Markram, Lübke, Frotscher, \& Sakmann, 1997). That is, whenever the population made an incorrect decision the weights between neurons with similar response patterns generated by stimuli $\Phi_{\text {ref }}$ and $\Phi_{\text {probe }}$ were weakened in order to sensitize the population to stimuli around the reference orientation. Specifically, after each trial the weights were updated as follows 
$\Delta w_{\theta_{i}, \theta_{j^{\prime}}}=-\eta V\left(\theta_{i}, \Phi_{\text {ref }}\right) V\left(\theta_{j}, \Phi_{\text {probe }}\right)$

where $\eta$ is the learning rate which is equal to zero for correct decisions and positive otherwise.

In some simulations, changes in synaptic connections can become stable as a consequence of consolidation processes. This was achieved simply by setting the learning rate of those connections to zero. The degree of consolidation was parametrically varied by allowing only a subset of zero, $25,50,75$, or 100 percent of those connections to undergo consolidation.

\section{Statistical Analysis}

Repeated measures ANOVAs, with part of experiment (A (i.e., baseline) and B or C (i.e., test)) and session as within-subject factors, were performed on the complete learning curve of fifteen sessions. In case of behavioral interference, analysis of the whole learning curve was expected to reveal an interaction, with the session effect differing per experiment part, showing slowed-down learning after intervening training. The beginning and end of learning curves, defined as the mean over first and last three thresholds of a learning curve respectively, were also compared with each other to assess interference. Five planned pairwise comparisons were performed. First, the beginning of the baseline and test learning curves was compared, as it was expected that for both backward and forward interference, the beginning of the learning curve, after additional training on different reference orientations, might be elevated compared to before additional training. Second, we also compared the end of the two learning curves as it was expected that backward and forward interference might also result in elevated asymptotic performance levels. Third, we compared the end of the learning curve before additional training to the beginning of learning after additional training, because we expected that for backward interference the beginning of the learning curve might fall back to untrained threshold levels. Finally, we also compared for both learning curves the beginning with the end of the curve to assess learning. Given the planned nature of the comparisons, we did not correct for multiple comparisons. If we nevertheless performed this correction, only two of the 17 comparisons we made in total for the 
three experiments ended up missing significance. This indicates that with or without correction, the t-tests showed largely the same pattern of results. We therefore report uncorrected $p$-vales for all planned pairwise comparisons. 


\section{Results}

We conducted a series of behavioral interference experiments with the aim to investigate whether the competition hypothesis put forth here is suited to account for interference during asymptotic learning or whether interference results from interruption of time-limited plasticity (consolidation hypothesis). We used an orientation discrimination task in which human observers were trained to distinguish small clockwise and counterclockwise deviations from a reference orientation. Previous work (Been et al., 2011) has shown that orientation discrimination at a $135^{\circ}$ reference orientation suffered from subsequent training at reference orientations $105^{\circ}$ and $165^{\circ}$ within the same $24 \mathrm{hs}$. Here, we tested whether performing the training at the $135^{\circ}$ reference orientation and at the $105^{\circ}$ and $165^{\circ}$ reference orientations in separate periods of several weeks would prevent interference, perhaps due to stronger consolidation. In order to gain a better insight into the underlying mechanism of interference and to generate testable predictions, we implemented a recurrent model of V1 orientation selectivity (Teich \& Qian, 2003). In this model, recurrent excitatory and inhibitory connections give rise to biologically plausible orientation tuning profiles of model neurons and hence to population response profiles related to differently oriented stimuli. Read-out of these reponse profiles allows for discrimination decisions to be made at a higher hierarchical level (implemented here in terms of signal detection theory; see Methods for details). As stimuli become more similar, individual neurons generate similar responses for each stimulus thus leading to undifferentiable population response profiles and low discrimination performance. Performance for highly similar stimuli can improve with experience, however, if neurons sharpen their tuning curves thereby allowing them to produce larger differences in firing rates for these stimuli.

In the model implemented here, the recurrent connections underlying tuning properties were adapted by an anti-Hebbian learning rule (Adini, Sagi, \& Tsodyks, 2002; Koch, Ponzo, Lorenzo, Caltagirone, \& Veniero, 2013; Markram, Lübke, Frotscher, \& Sakmann, 1997) in response to training. For example, if a stimulus objectively rotated counterclockwise from the reference is judged as clockwise rotated, and if another stimulus objectively rotated clockwise from the reference is 
judged as counterclockwise rotated, then the participant will receive negative feedback in both cases. The anti-Hebbian learning rule is implemented by sending a negative feedback signal to neurons that respond to varying degrees to both stimuli. This will weaken the recurrent connections between these neurons thus separating the population response profiles for each stimulus (see Methods for details). The result at the individual neuron level is a tuning curve with a steeper flank facing the trained reference orientation. While this mechanism leads to improved decision performance for small orientation differences at the trained reference orientation, population response profiles for orientation differences around reference orientations rotated merely $\pm 30^{\circ}$ away from the trained reference orientation will become more similar. This is due to the fact that synaptic changes are asymmetric as a single neuron only weakens its recurrent connections with neurons that contribute to one flank of its tuning curve (facing the trained reference orientation). Recurrent connections with neurons contributing to the other flank remain unchanged. The resulting synaptic imbalance effectively broadens the flank of a neuron's tuning curve facing away from the trained reference orientation and reduces its ability to distinguish between orientations falling on that flank. Thus, adaptive sharpening of neurons' tuning flank at the reference orientation is accompanied by maladaptive broadening of their tuning flank away from the reference orientation. Therefore, when different tasks simultaneously induce the same flanks of neuron's tuning curves to sharpen and to broaden, the resulting competition leads to behavioral interference.

This mechanism can account for asymptotic perceptual learning by Been et al. (2011). Specifically, we were able to reproduce the asymptotic perceptual learning effect reported by the investigators by fitting three free model parameters to the data: initial discrimination performance at the start of asymptotic learning, a dimensionless constant $k$ regulating the variance of neurons' firing rates, and the positive learning rate $\eta$ controlling the weight change in the anti-Hebbian learning process. Initial discrimination performance was set to 1.5 , the average $\ln$ transformed $84 \%$ JND observed at the beginning of asymptotic learning (starting from session 8) in Been et al. (2011). Since constant $k$ regulates the variance of firing rates of neurons, it affects the signal-to-noise ratio in the signal detection 
process and hence the baseline discrimination performance. This parameter was set to 1.55 as this leads to good fit with mean performance in the data of Been et al. (2011). Finally, the learning rate $\eta$ was set equal to $1.8 \times 10^{-9}$. When the model was trained using the learning paradigm of Been et al. (2011), it produced JNDs comparable to those reported by the investigators (see Figure 3 - dark gray lines).

Furthermore, when the model was exposed to the interference paradigm employed by Been et al. (2011) with the above-described parameter set, it failed to improve its performance at the $135^{\circ}$ reference orientation. This result is in accordance with the empirical data (Figure 3A, B - light gray lines). However, while the slopes of model and empirical data were comparable, the intercept for the interfered condition was higher in the empirical data as compared to the model data. This is because in the model, we chose to set the mean baseline discrimination performance $k$ to the same value $(k=1.55)$ in the control training condition and in the training condition including interference. When we ran the model with a mean baseline discrimination performance that captured performance levels after 7 sessions $(k=3.7)$ of interference between training at $135^{\circ}$ and $105^{\circ} / 165^{\circ}$, continued exposure of the model to the two competing tasks $\left(135^{\circ}\right.$ vs $\left.105^{\circ} / 165^{\circ}\right)$ confirmed the lack of improvement at the $135^{\circ}$ reference orientation. In the remaining simulations,
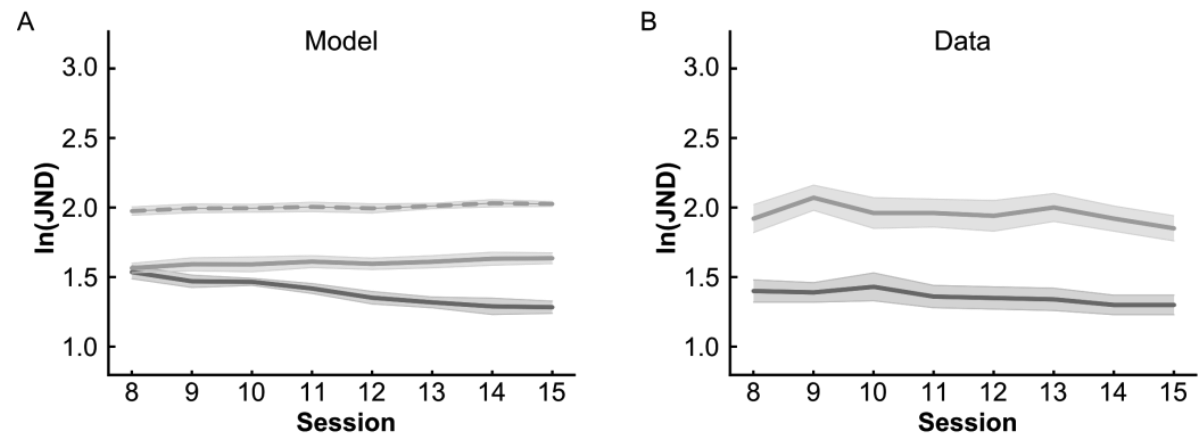

Figure 3. Modelling $(\boldsymbol{A})$ and behavioral data $(\boldsymbol{B})$ of asymptotic performance (dark grey line) and behavioral interference (light grey line) as reported in Been et al. (2011). The dashed light grey line accounts for worsened baseline performance due to early interference, which is not used for further simulation as it adds a confounding interference effect to the simulations. 
effects of baseline training and experimental training will be tested starting with the same level of discrimination performance.

While a competition mechanism like the one implemented in our model is able to explain behavioral interference, it is conceivable that interference effects can be counteracted by a sufficiently large population wherein different subpopulations can separate their response profiles in a manner adaptive for one or the other task. Such a scenario would benefit from consolidation in which synaptic connectivity changes in subsets of neurons would become fixed, thus forming unique subpopulations carrying different memory traces. In order to simulate the establishment of subpopulations as a consequence of consolidation, the model allowed a varying proportion of connections to 'freeze' once they have been set by experience. These connections then become immune to further experience, and hence might diminish behavioral interference. We will provide predictions from our model with and without consolidation and test them against empirical data in our 15-session blocked learning experiment. We first describe control data (green frames in Figure 2) in which under none of the proposed hypotheses interference is expected. We then describe two experiments, in which interference may (or may not) be expected (red and blue frames in Figure 2).

\section{Control experiment}

In this experiment, we employed a paradigm in which training at a $135^{\circ}$ reference orientation was followed by training at a dissimilar $\left(45^{\circ}\right)$ reference orientation, after which training continued at the $135^{\circ}$ reference orientation. Note that each stage lasted for 15 sessions and was finished before starting the next stage (see Figure 2). This experiment was included because data from Been et al. (2011) had shown that no behavioral interference was observed when alternating training at orthogonal stimuli. In principle, the training at the $135^{\circ}$ reference orientation on the one hand, and at the $45^{\circ}$ references on the other hand, should recruit distinct populations of neurons. Accordingly, we did not expect any interference when training at orthogonal reference orientations was performed in a sequential manner. 


\section{Behavioral data}

Here, we investigated whether the skill resulting from 15 baseline training sessions at the $135^{\circ}$ reference orientation (Part A, Figure 4) would be negatively affected by 15 sessions of training in the same location at an orthogonal reference orientation (Part B, Figure 4). This was tested by re-initiating training at the $135^{\circ}$ reference orientation (Part C, Figure 4). A repeated measure ANOVA with part of experiment (Part A, Part C) and session (1-15) as within-subject factors revealed no significant main effect of experiment part $\left(F_{(1,5)}=3.050, p=.141\right)$ but a significant main effect of session $\left(F_{(14,70)}=11.438, p<.001\right)$ and a significant interaction $\left(F_{(14,70)}=3.688, p\right.$ $<.001$ ) (Figure 5A). This data pattern was compatible with the interpretation that intervening training at an orthogonal reference orientation $\left(45^{\circ}\right)$ did not affect the performance gains obtained by earlier training at the $135^{\circ}$ reference for 15 sessions.

This was confirmed by a comparison of the beginnings and ends of the learning curves (defined as the mean of the first or last three thresholds) in Parts A and C (summarized in Figure 5B). Mean threshold levels for start and end of learning curves differed from each other within Part A $\left(t_{(5)}=9.155, p<.001\right)$ but not within Part $\mathrm{C}\left(t_{(5)}=2.174, p=.082\right)$ of the experiment, showing there was learning in Part

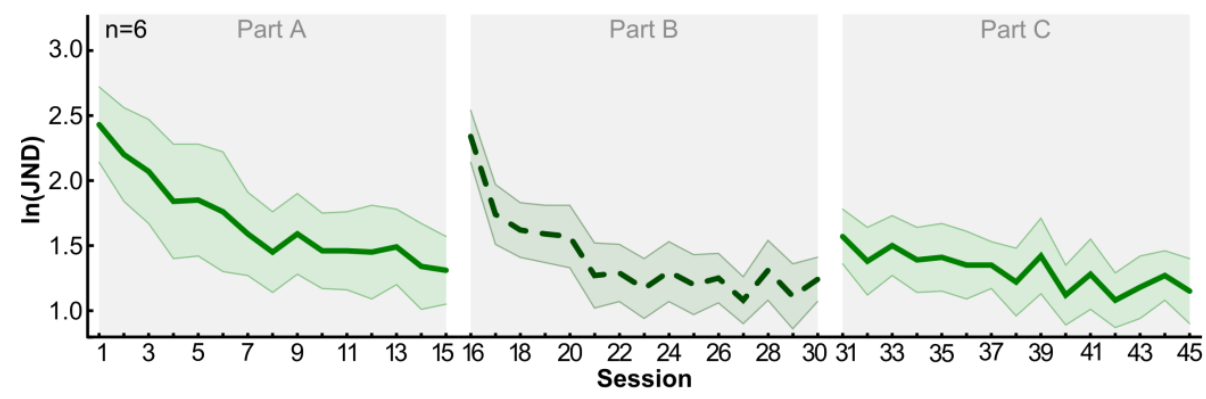

Figure 4. Conditions belonging to the experiment indicated by green frames in Figure 2. In Part A initial baseline training is performed on an oblique reference orientation $\left(135^{\circ}\right.$, baseline condition) and then followed in Part B by training at a non-interfering reference orientation $\left(45^{\circ}\right.$ - dashed line, orthogonal condition) in the same quadrant. Finally, in Part C participants are re-trained at the oblique reference orientation $\left(135^{\circ}\right.$, test condition). To test whether training prior to control training at a $45^{\circ}$ reference orientation changes the resulting learning curve in Part C (test condition), it is compared to the learning curve without any prior interference in Part A (baseline condition). Colored areas represent SEM. 

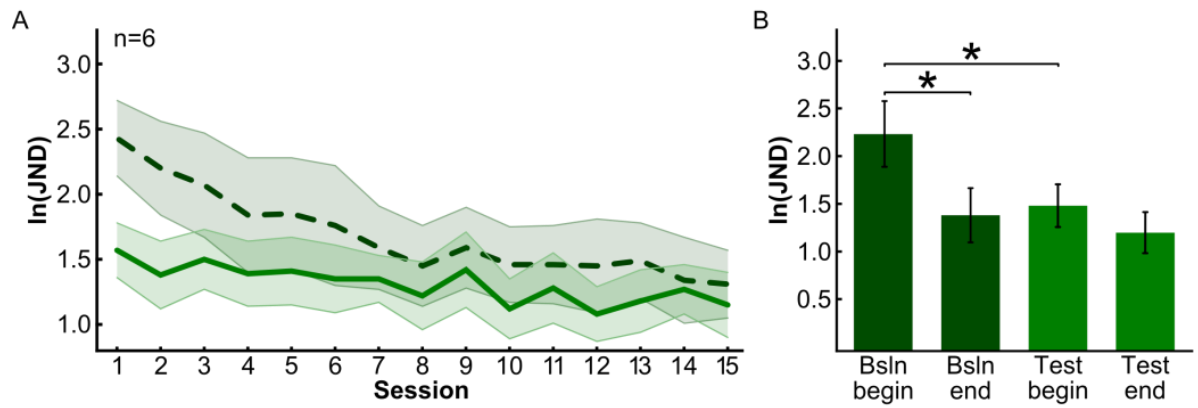

Figure 5. A, Learning curves for baseline (dashed dark green line) and test condition (light green line) over 15 sessions of training. $\boldsymbol{B}$, Mean threshold levels of beginning and end of learning for baseline (dark green bars) and test conditions (light green bars). $(* p<.05)$

$\mathrm{A}$, but not in Part $\mathrm{C}$. The beginnings of the learning curves in Part $\mathrm{A}$ and $\mathrm{C}$ were significantly different $\left(t_{(5)}=3.750, p=.013\right)$, confirming the idea that learning is not interfered by intervening training at an orthogonal reference orientation. Importantly, the end of the learning curve of Part A did not differ significantly from the beginning of the learning curve in Part $\mathrm{C}\left(t_{(5)}=-0.804, p=.458\right)$. Moreover, the ends of the learning curves in Parts $\mathrm{A}$ and $\mathrm{C}$ did not differ from each other significantly $\left(t_{(5)}=0.861, p=.429\right)$. This analysis further confirms the lack of interference between two subsequent 15-session blocks of training at orthogonal reference orientations.

\section{Comparison of Model and Data}

In line with Been et al. (2011), we expected that training at a given reference orientation would not be interfered with by additional training in the same location at an orthogonal reference orientation. In the model, performance after orthogonal training was even better than before intervening training with the orthogonal reference orientation, thus showing the lack of any interference. Figure 6 shows the learning performance of the model over the final eight sessions of asymptotic learning (Figure 6A) as compared to behavioral results (Figure 6B). Note that the model is limited to the prediction of asymptotic learning as it deals with tuning changes in early visual areas thought to occur only during the final training stages (De Weerd, Pinaud, \& Bertini, 2006; Karni, 1996). The performance produced by 
the model is in good agreement with the learning performance observed in our participants. To compare model and behavioral data, difference curves between baseline (i.e. training before orthogonal training) and test (after training at stimuli differing by $45^{\circ}$ ) were calculated for both model and behavioral data (Figure 6C). The residual mean squared error (RMSE) between both difference curves was calculated and tested for significance based on a null-distribution of difference curves created by bootstrapping. Specifically, we used a block bootstrap to keep temporal relations intact and performed randomization only within subjects. The null-distribution was then created from 10000 difference curves obtained from random baseline and test samples. These samples were created by first randomly drawing from the subject pool with replacement and then randomly placing one of this subject's two learning curves in the baseline and the other in the test sample repeatedly until each sample contained data of six subjects. The resulting fit of the model and empirically observed difference between baseline (Part A) and test (Part C) learning curves was good but failed to reach significance $(\mathrm{RMSE}=0.13, p$ $=.080$ ). The null distribution in the bootstrap was created under the assumption that there is no difference between learning curves for part $\mathrm{A}$ and part $\mathrm{C}$ in our experiments. Consequently, the match between difference curves for empirical data and as predicted by the model is difficult to establish statistically if empirical difference curves are close to zero. In light of this a $p$-value of .08 is rather good
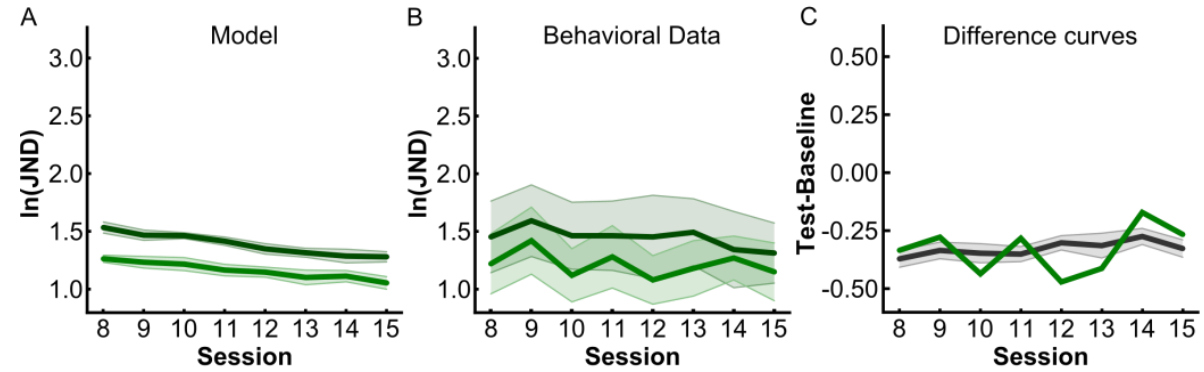

Figure 6. A, Modeling data for asymptotic learning (i.e. session 8-15). B, Behavioral data for asymptotic performance of 6 participants. The dark green line belongs to baseline training (Figure 2, Part A, green), whereas the light green line belongs to re-training after intervening training at orthogonal stimuli (Figure 2, Part C). $\boldsymbol{C}$, Difference curves between light and dark green line were calculated for modeling data (grey) and behavioral data (green). 
when considering the small difference between learning curves in Parts A and C in the control experiment.

\section{Backward Interference (blue)}

In this experiment we employed a backward interference paradigm, in which baseline training at a $135^{\circ}$ reference orientation was followed by intervening training at similar $\left( \pm 30^{\circ}\right)$ reference orientations. The expected interference was tested by returning to training at the $135^{\circ}$ reference orientation. Note that each stage lasted for 15 sessions and was finished before starting the next stage (see Figure 2).

We tested whether a long period of training at the $135^{\circ}$ reference orientation (15 sessions) would make the resulting memory trace immune to any effects of subsequent training at the $105^{\circ}$ and $165^{\circ}$ reference orientations. In the simplest implementation of a consolidation model, upon completion of training at the $135^{\circ}$ reference orientation, a proportion of synapses in the relevant network would have stabilized. Depending on model parameters, one could under this scenario perhaps still expect some slower learning at the $105^{\circ}$ and $165^{\circ}$ reference orientations, but for the $135^{\circ}$ reference orientation the prediction would be that the skill learned in Part A would survive the additional training in Part B, resulting in initial thresholds in Part $\mathrm{C}$ for the $135^{\circ}$ reference equaling the thresholds at the end of the $135^{\circ}$ reference learning curve in Part A. Alternatively, no synapses consolidate. In this case, despite a long period of training in Part A at the $135^{\circ}$ reference orientation (intermixed with a number of days of rest - weekends and some occasional week days without testing), we would expect interference induced by the subsequent training period at the $105^{\circ}$ and $165^{\circ}$ reference orientations in the same location (Part B) to produce elevated thresholds once thresholds at the $135^{\circ}$ reference orientation are retested in that location during Part $\mathrm{C}$. Hence, the stronger the role of consolidation, the smaller is the expected interference.

Note that 'immunity' from interference could also be compatible with a consolidation scenario in which potential redundancies in the tuning properties of a large population of neurons might be utilized to form a smaller subpopulations of neurons in which a separate, stable memory trace could be formed for the $135^{\circ}$ reference orientation, while resetting connections among other neurons not involved 
in the memory trace. In this manner, although in principle the training at the $135^{\circ}$ reference orientation on the one hand, and at the $105^{\circ}$ and $165^{\circ}$ references on the other hand, would recruit largely overlapping populations, the subset of neurons and connections involved in the memory trace for the $135^{\circ}$ reference would be unaffected by subsequent training in the same location at the $105^{\circ}$ and $165^{\circ}$ reference orientations, and the training at the latter reference orientations itself might result in segregated and stable memory traces. In this scenario, retesting at the $135^{\circ}$ reference orientation (Figure 2, Part C, E135) in the same location as original training also would yield thresholds equal to the asymptote obtained after original learning (Figure 2, Part A, E135).

\section{Behavioral data for Backward Interference - Experimental (blue)}

In three testing periods (blue framed panels in Figure 2), we obtained first a learning curve at the $135^{\circ}$ reference orientation (Part A), then learning curves at the $105^{\circ}$ and $165^{\circ}$ references (Part $\mathrm{B}$ ), and then a retest at the $135^{\circ}$ reference (Part $\mathrm{C}$ ), in fact yielding a second learning curve at that reference. A comparison in Figure 7 of the beginning of testing in Part $\mathrm{C}$ with the end of testing in Part A suggests a negative

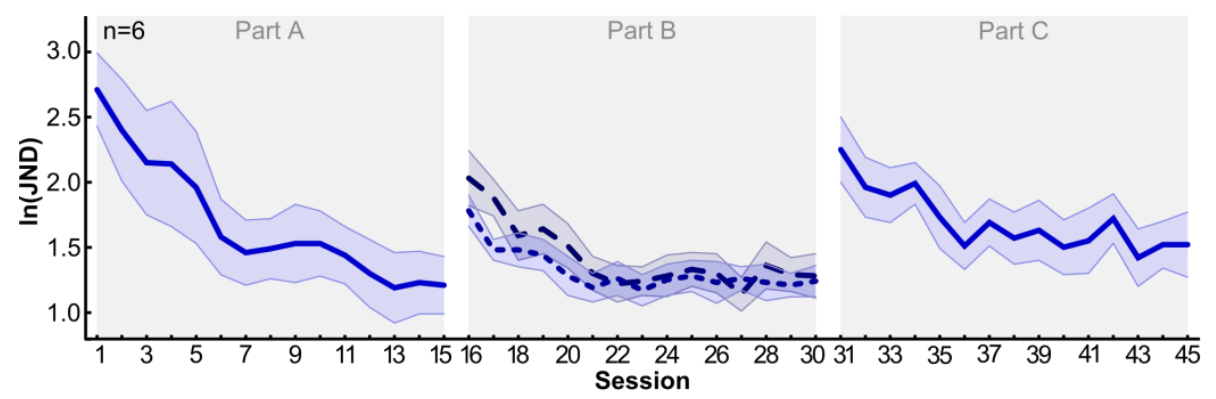

Figure 7. Conditions belonging to the experiment indicated by blue frames in Figure 2 (backward interference). In Part A training was performed on an oblique reference orientation $\left(135^{\circ}\right)$ and followed in Part B by intervening training at similar $\left( \pm 30^{\circ}\right)$ reference orientations $\left(105^{\circ}\right.$ - dashed line; $165^{\circ}$ - dotted line) in the same quadrant. Finally, in Part C participants are re-trained at the oblique reference orientation. To test whether performance on the $135^{\circ}$ reference orientation in Part $\mathrm{C}$ (test condition) was affected by intervening training at a $105^{\circ} / 165^{\circ}$ reference orientations in Part $\mathrm{B}$, it is compared to the learning curve without any prior interference in Part A (baseline condition). 
impact of the intermediary training at the $105^{\circ}$ and $165^{\circ}$ reference orientations on orientation discrimination at the $135^{\circ}$ reference in Part $\mathrm{C}$.

A repeated measures ANOVA with experimental part (Part A, Part C) and session (1-15) as within-subject factors revealed a significant main effect of session $\left(F_{(14,70)}=16.625, p<.001\right)$, a significant interaction $\left(F_{(14,70)}=2.658, p=.004\right)$, and a non-significant main effect of experimental Part $\left(F_{(1,5)}=0.003, p=.960\right)$ (Figure 8A). To further explore effects of the intervening training in Part B, we tested differences among beginnings and ends of learning curves in Parts A and C. Mean threshold levels (Figure 8B) for start and end of learning curves differed from each other in baseline learning in Part $\mathrm{A}\left(t_{(5)}=7.507, p=.001\right)$ but not in re- training in Part C $\left(t_{(5)}=2.306, p=.069\right)$, further supporting the observed interaction in the complete dataset. A main prediction of the competition hypothesis was that the beginning of the learning curve at the $135^{\circ}$ reference in Part $\mathrm{C}$ would be elevated compared to the end of the learning curve in Part A. This trend was visible in the data and also highly significant $\left(t_{(5)}=-4.308, p=.008\right)$. The beginning of the learning curve in Part $\mathrm{C}$ was not significantly different from the beginning of the learning curve in Part A $\left(t_{(5)}=1.340, p=.238\right)$. There appeared to be a tendency for the end of the learning curve in Part $\mathrm{C}$ to be elevated compared to the end of the learning curve in Part A, however, this was not significant $\left(t_{(5)}=-2.075, p=.093\right)$. Taken together, these findings show that the training at reference orientations
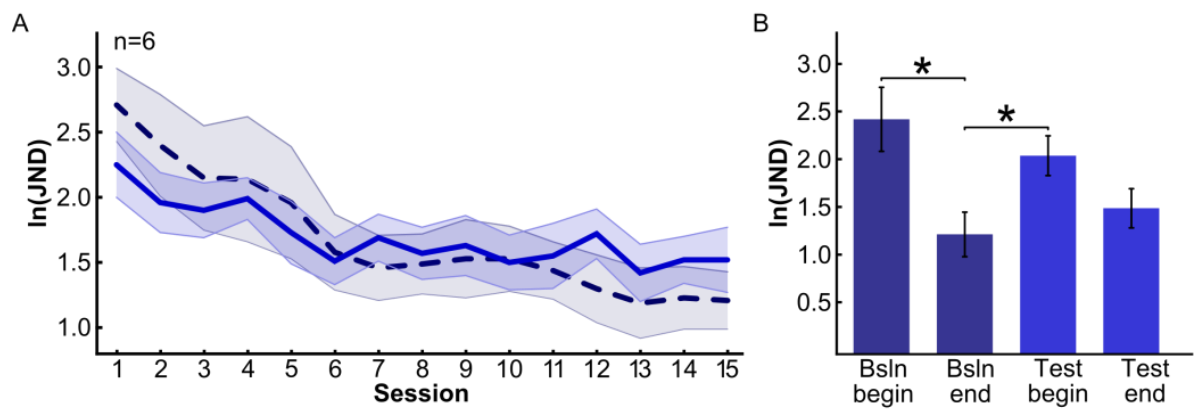

Figure 8. $\boldsymbol{A}$, Learning curves for baseline (dashed dark blue line) and test condition (light blue line) over 15 sessions of training. $\boldsymbol{B}$, Mean threshold levels of beginning and end of learning for baseline (dark blue bars) and test conditions (light blue bars). $(* p<.05$ ) 
differing by $\pm 30^{\circ}$ (Part B) from the $135^{\circ}$ reference used in preceding baseline training (Part A), severely interfered with performance at the $135^{\circ}$ reference,requiring relearning (as observed in Part C). It can thus be concluded that even 15 sessions of training (in a period of 4-5 weeks) are not sufficient to protect against interference from competing tasks.

\section{Comparison of Model and Data}

The model prediction of interference was done by comparing asymptotic learning in Part A (baseline) and Part C (test). Model predictions of an overlapping-populations scenario for asymptotic learning are shown in Figure 9A and strongly resemble those observed for the experimental data (Figure 9B). Difference curves between baseline (Part A) and test (Part C) were calculated for both model and behavioral data (Figure 9C). For these comparisons our model predicts very small differences between baseline and test learning curves. The residual mean squared error between model predictions and empirical results is low (RMSE: $0.15, p=.389$ ), signifying a good fit, but not significant for the same reasons as in the control experiment. However, as more synapses undergo consolidation an increasing difference is predicted between learning curves of Parts $\mathrm{A}$ and $\mathrm{C}$ with better performance
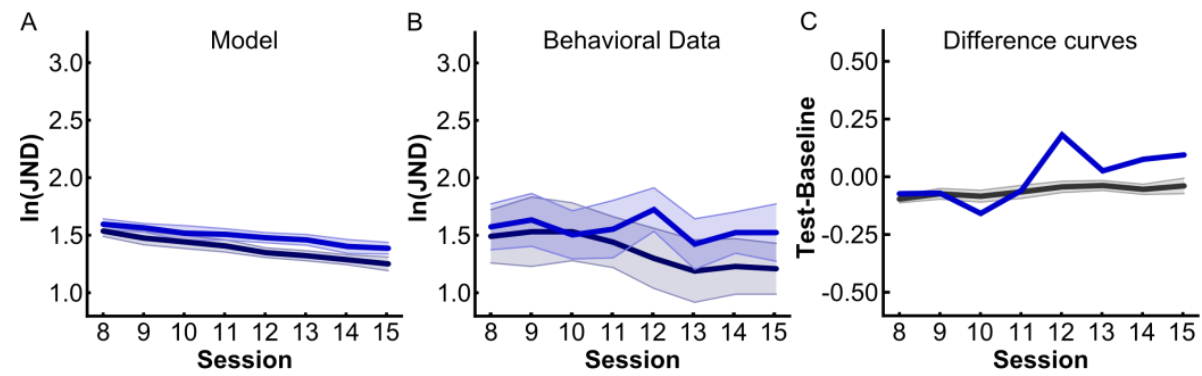

Figure 9. A, Modeling data for asymptotic learning (i.e. session 8-15) for Part A and Part C in the backward interference experiment. $\boldsymbol{B}$, Behavioral data for asymptotic performance of 6 participants. The dark blue line belongs to baseline training (before intervening training at stimuli differing by $\pm 30^{\circ}$; Figure 2 , Part A, blue), whereas the light blue line belongs to test training (after intervening training at stimuli differing by $\pm 30^{\circ}$; Figure 2, Part C, blue). $\boldsymbol{C}$, Difference curves between baseline and test learning curves were calculated for modeling data (grey) and behavioral data (blue). 
expected in Part C. As this difference is not evident in the data (Figure 10), residual mean squared errors as well as $p$-values increase for these predictions (25\%: RMSE $=0.17, p=.532 ; 50 \%: \operatorname{RMSE}=0.20, \mathrm{p}=.679 ; 75 \%: \mathrm{RMSE}=0.23, \mathrm{p}=.757 ; 100 \%$ : RMSE $=0.27, \mathrm{p}=.826$ ) .differing by $\pm 30^{\circ}$; Figure 2 , Part A, blue), whereas the light blue line belongs to test training (after intervening training at stimuli differing by $\pm 30^{\circ}$; Figure 2, Part C, blue). $\boldsymbol{C}$, Difference curves between baseline and test learning curves were calculated for modeling data (grey) and behavioral data (blue).
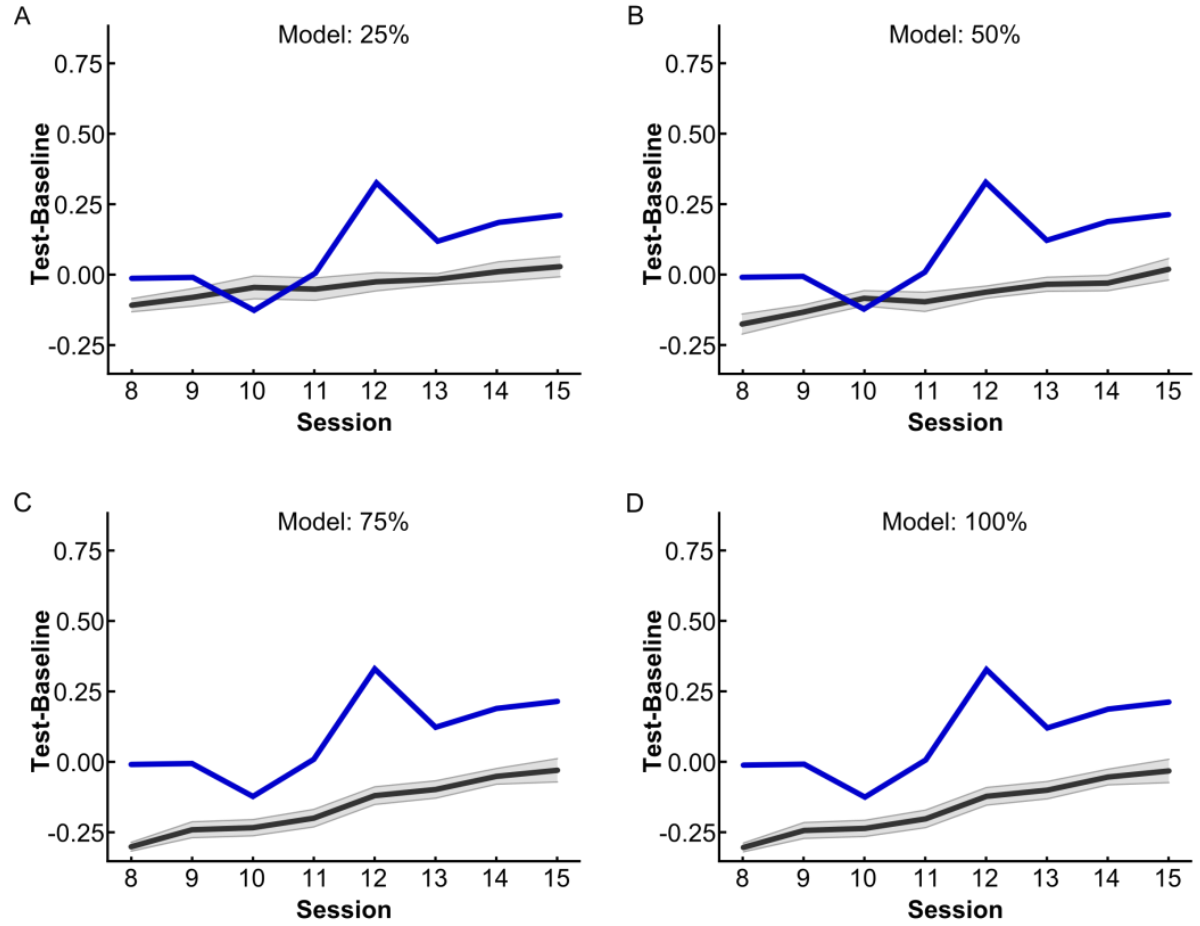

Figure 10. Difference curves (based on baseline and test curve of the backward interference experiment) for different degrees of consolidation: $25 \%(\boldsymbol{A}), 50 \%(\boldsymbol{B}), 75 \%(\boldsymbol{C})$, and $100 \%$ $(\boldsymbol{D})$.

\section{Behavioral data - comparing Backward Interference and Control experiments}

In further analysis, we tested the magnitude of the interference effect in the present backward interference experiment by comparing thresholds collected in Part $\mathrm{C}$ for the present experiment (Fig. 2, Part C, blue frame $\mathrm{E}_{135}$ ) with threshold collected in Part $\mathrm{C}$ for the control experiment (Fig. 2, Part $\mathrm{C}$, green frame $\mathrm{C}_{135}$ ). Figure 11A 
compares the 15 sessions of testing in Part $\mathrm{C}$ for the two experiments, and shows a clear trend for thresholds in the backward interference experiment to exceed those in the control experiment. A repeated measures ANOVA with experiment (backward interference, control) and session (1-15) as within-subject factors revealed a significant main effect of session $\left(F_{(14,70)}=3.797, p<.001\right)$, a significant main effect of experiment $\left(F_{(1,5)}=38.587, p=.002\right)$, and a significant interaction $\left(F_{(14,70)}=\right.$ 2.968, $p=.001)$. Pairwise comparison revealed that most of the significant differences (and the strongest ones) were found for the earlier sessions, in line with the interaction yielded by ANOVA (session 1-4: $p<.008$; session 5: $p=.077$; session 6: $p=.246$; session 7-8: $p<.011$; session 9: $p=.096$; session 10-13: $p<$ .032 ; session 14: $p=.051$; session 15: $p=.080$; all $p$-values are uncorrected). This indicates that more learning is taking place in Part $\mathrm{C}$ in the backward interference experiment than in the control experiment.

To further explore effects of the intervening training in Part B on testing in Part C, we tested differences among beginning and ends within the learning curves in Parts $\mathrm{C}$ for the Control experiment (Figure 11B, green bars) and for the Backward Interference experiment (Figure 11B, blue bars). The differences between mean threshold levels for start and end of learning curves did not reach significance in
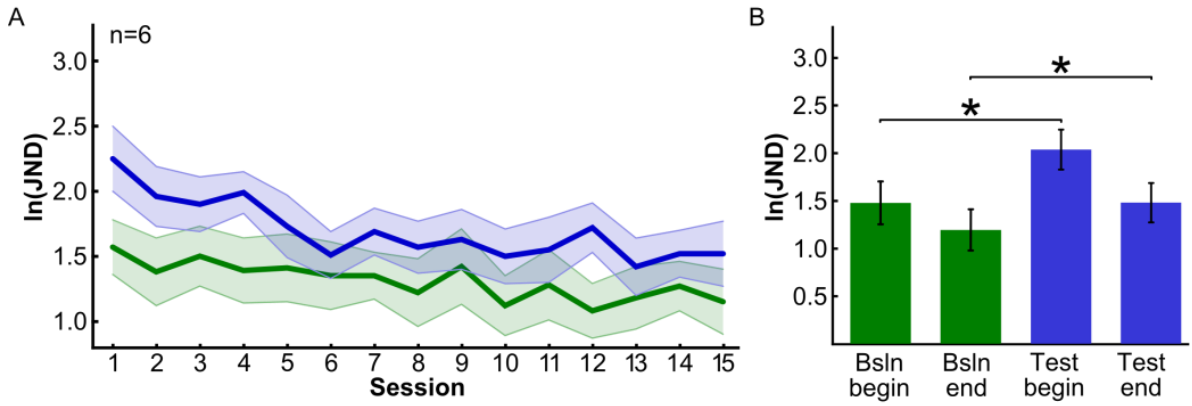

Figure 11. Comparison of performance in Part $\mathrm{C}$ in backward interference and control experiments. $\boldsymbol{A}$, Learning curves for test conditions in the Control experiment (green line) and the Backward Interference experiment (blue line) over 15 sessions of training. $\boldsymbol{B}$, Mean threshold levels of beginning and end of learning for test condition in the Control experiment (green bars) and the Backward Interference experiment (blue bars). $(* p<.05)$ 
both experiments (Control: $t_{(5)}=2.174, p=.082$; Backward Interference: $t_{(5)}=$ 2.306, $p=.069$ ), indicating that the different learning rate in the two experiments during Part $\mathrm{C}$ revealed by ANOVA (see previous paragraph) was not captured by the present choice of data included in the t-tests. The beginning of the learning curve in the Backward interference experiment was significantly higher than in the Control experiment $\left(t_{(5)}=7.723, p=.001\right)$. The end of the learning curve in the Backward Interference experiment also remained significantly elevated compared to the end of the learning curve in the Control experiment $\left(t_{(5)}=3.157, p=.025\right)$. Taking the results from ANOVA and t-tests together, it can be concluded that in the backward interference experiment, the intervening training at reference orientations differing by $\pm 30^{\circ}$ from the $135^{\circ}$ reference (Part B) interfered with the skill memory induced by the preceding baseline training (Part A), requiring retraining at the $135^{\circ}$ reference (as observed in Part C). This further supports the conclusion that even 15 sessions of training (in a period of 4-5 weeks) are not sufficient to protect against interference from competing tasks.

Note that the differences between the data in Part $\mathrm{C}$ of backward interference and control experiments cannot be due to left/right hemifield differences, as in half of the participants all conditions were mirrored around the vertical compared to the other half of participants. However, it could be argued that the difference between the backward

interference and control experiments reflect in part a lower visual field advantage (Fuller, Rodriguez, \& Carrasco, 2008; Montaser-Kouhsari \& Carrasco, 2009; Talgar $\&$ Carrasco, 2002). This possibility is contradicted by data in Part A, which shows lower thresholds at the end of learning in the upper quadrant (backward interference experiment, see Fig 7A) than in the lower quadrant (control experiment, see Fig 4A).

Comparison of Model and Data - comparing Backward Interference and Control experiments

An alternative model prediction of interference was done by comparing learning in Part $\mathrm{C}$ in the control experiment (when intervening learning was done at a reference orientation orthogonal to that used in baseline) with learning in Part $\mathrm{C}$ in the present experiment (when intervening learning was done at reference orientations deviating by $\pm 30^{\circ}$ from that used in baseline). Model predictions of an overlapping- 
populations scenario for asymptotic learning are shown in Figure 12A and strongly resemble those observed for the experimental data (Figure 12B). Difference curves between control (i.e. after training at dissimilar stimuli differing by $90^{\circ}$, green condition) and interference (after training at similar stimuli differing by $\pm 30^{\circ}$, blue condition) were calculated for both model and behavioral data (Figure 12C). The resulting fit of the model prediction of differences to the empirically observed differences was significant (RMSE: $0.13, p=.049$ ). To test in how far consolidation mechanisms might explain our data, we used our model to predict the outcome of a behavioral interference experiment for the cases that $25,50,75$, or 100 percent of synaptic changes resulting from training at the reference orientation consolidate before training at interfering orientations. Consolidation in this case is assumed to occur after completion of all 15 training sessions for the $135^{\circ}$ reference orientation. A proportion of zero percent represents the case in which no consolidation occurs whereas the other cases render an increasing degree of synaptic changes permanent. Both, synapses undergoing and not undergoing consolidation continue to reflect the recently formed memory trace. However, those synapses not undergoing
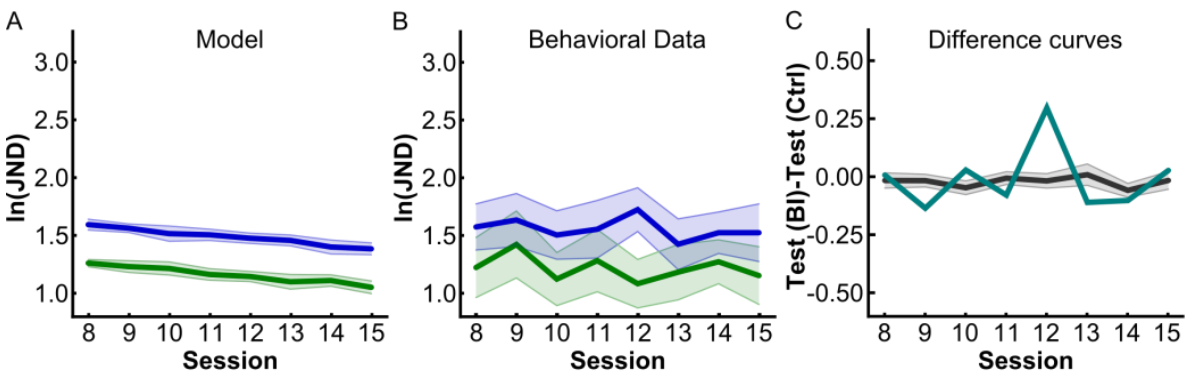

Figure 12. A, Modeling data for asymptotic learning (i.e. session 8-15) in backward interference and control experiments in Part C. B, Behavioral data for asymptotic performance of 6 participants. The green line belongs to the control experiment (after intervening training at orthogonal stimuli; Figure 2, Part C, green), whereas the blue line belongs to backward interference experiment (after intervening training at stimuli differing by $\pm 30^{\circ}$; Figure 2, Part C, blue). $\boldsymbol{C}$, Difference curves between blue and green line were calculated for modeling data (grey) and behavioral data (blue). Note that the model shown here fares better than the models shown in Figure 10C where the difference curve was based on baseline and test curves of the backward interference experiment. 
consolidation can subsequently be modified as synapses are recruited to form a new memory trace in response to training at a different reference orientation. The resulting model predictions are shown in Figure 13. All difference curves based on a consolidation model fare much worse $(25 \%$ : RMSE $=0.14, p=.109 ; 50 \%: \mathrm{RMSE}=$ $0.16, p=.238 ; 75 \%: \mathrm{RMSE}=0.19, p=.395 ; 100 \%: \mathrm{RMSE}=0.23, p=.602)$ than the model with $0 \%$ consolidation shown in Figure 12C. As expected the effect of training at interfering reference orientations decreases as the proportion of synapses coding the initial memory trace undergoing consolidation increases.
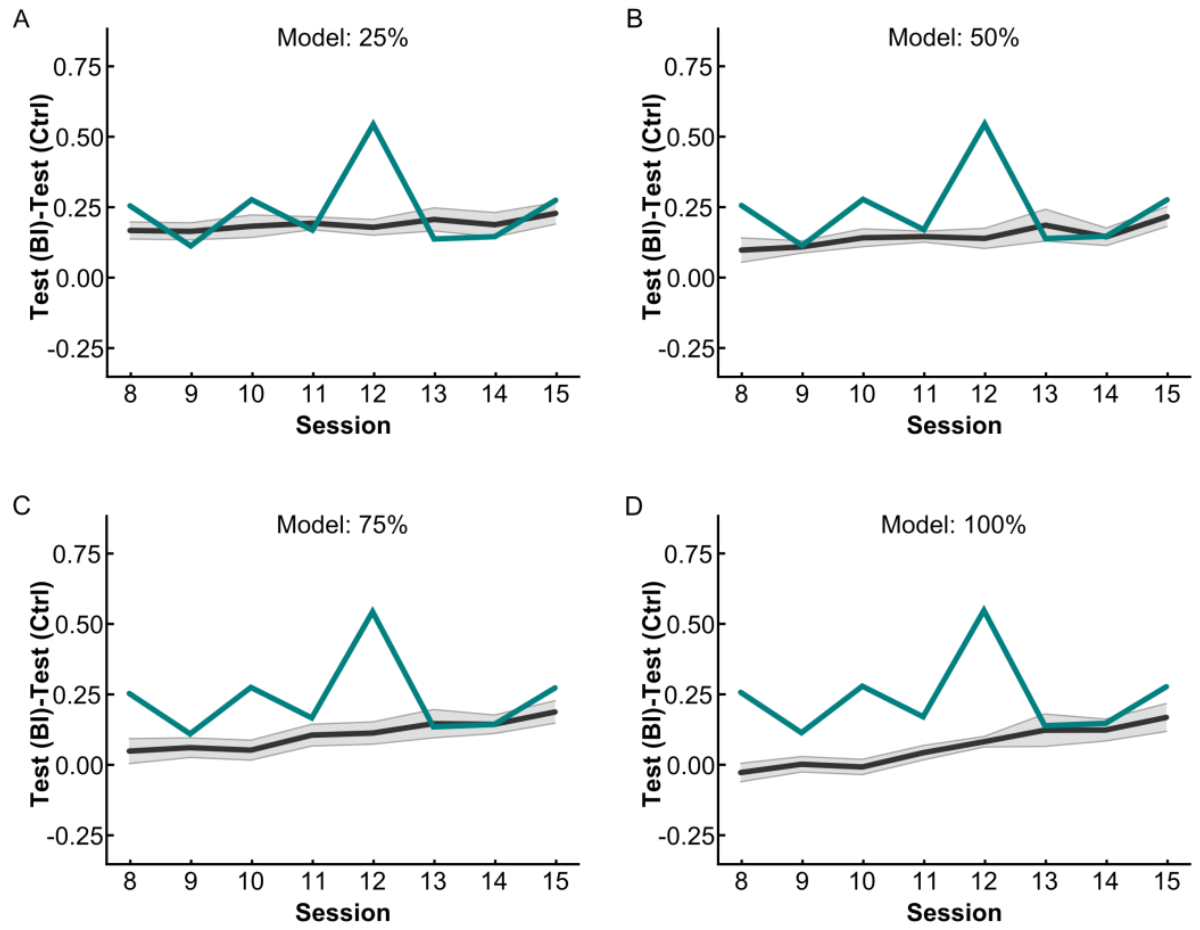

Figure 13. Difference curves (for test learning curves of the backward interference experiment and the control experiment) for different degrees of consolidation: $25 \%(\boldsymbol{A}), 50 \%$ $(\boldsymbol{B}), 75 \%(\boldsymbol{C})$, and $100 \%(\boldsymbol{D})$. Note that all models shown here fare worse than the model shown in Figure 10C with 0\% consolidation. BI: backward interference experiment; Ctrl: Control experiment. 


\section{Forward Interference (Red)}

In this experiment, we employed a forward interference paradigm to investigate whether, in accordance with the competition hypothesis, training at reference orientations $105^{\circ}$ and $165^{\circ}$ puts constraints on further orientation training at $135^{\circ}$.

\section{Behavioral data for Forward interference (red)}

To investigate how training at the $105^{\circ}$ and $165^{\circ}$ references (Part A, Figure 14) affected subsequent learning at the $135^{\circ}$ reference (Part B, Figure 14), we compared the latter learning curve to a baseline learning curve in a different quadrant without prior training at any orientation (blue line Part A, Figure 14). A repeated measure ANOVA with experimental part (Part A - blue, Part B - red) and session (1-15) as within-subject factors revealed a non-significant main effect of experimental part $\left(F_{(1,5)}=1.321, p=.302\right)$, a significant main effect of session $\left(F_{(14,70)}=23.067, p<\right.$ $.001)$ and a significant interaction $\left(F_{(14,70)}=2.173, p=.017\right)$ (see also Figure 15A). The interaction was due to differences in thresholds between baseline and test data in sessions $13\left(t_{(5)}=-4.313, p=.008\right.$, uncorrected) while all other comparisons were not significant (all $p>0.5$ ). Mean threshold levels (Figure 15B) for start and end of learning curves differed from each other for both baseline $\left(t_{(5)}=7.507, p=.001\right)$

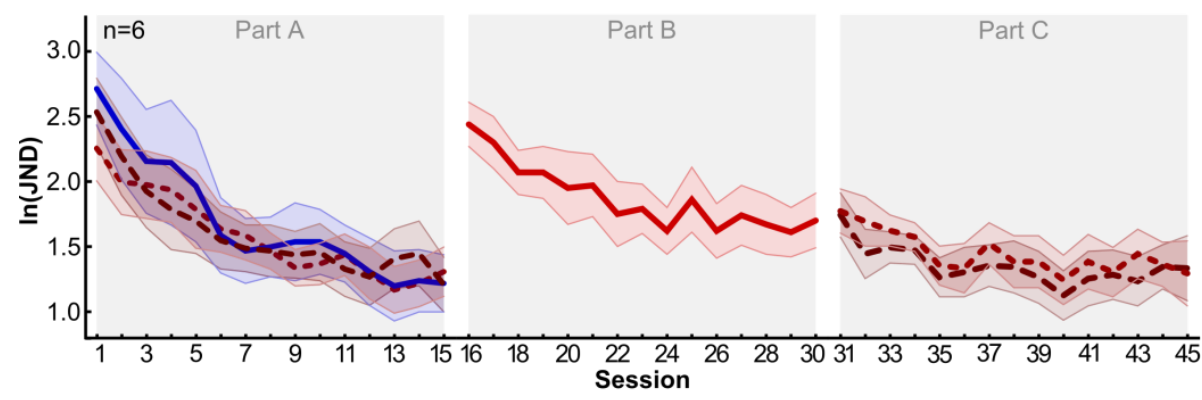

Figure 14. Conditions belonging to the experiment indicated by red frames in Figure 2. In Part A training is performed on two interfering reference orientations $\left(105^{\circ}\right.$ - dashed line; $165^{\circ}$ - dotted line) and then followed in Part B by training at the oblique reference orientation $\left(135^{\circ}\right)$ in the same quadrant. To test whether prior interference changes the resulting learning curve in Part B, it is compared to a learning curve without any prior interference in another quadrant (Part A, shown in blue) which corresponds to the condition indicated by blue/red frames in Figure 2. Colored areas represent SEM. 

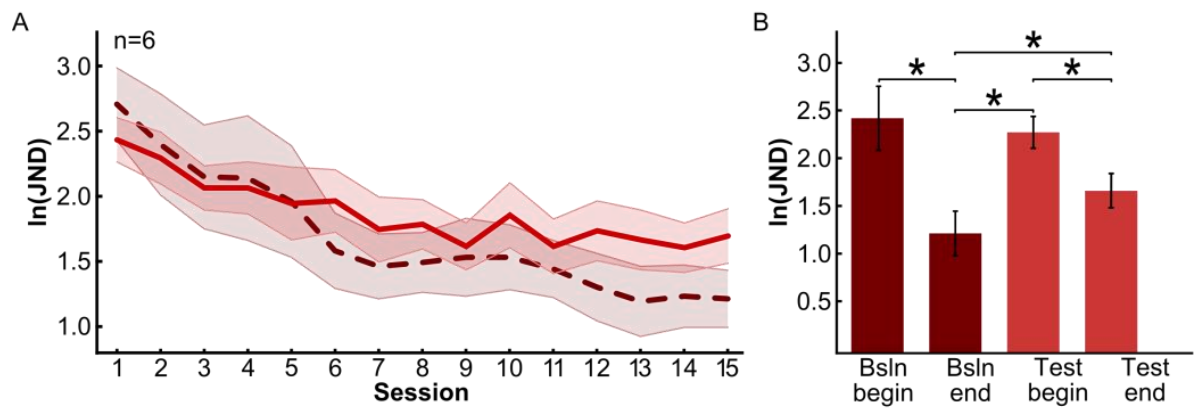

Figure 15. A, Learning curves for baseline (dashed dark red line) and test condition (light red line) over 15 sessions of training. $\boldsymbol{B}$, Mean threshold levels of beginning and end of learning for baseline (dark red bars) and test conditions (light red bars). $(* p<.05)$.

and test condition $\left(t_{(5)}=5.351, p=.003\right)$, confirming significant learning in both conditions. The beginning of learning curves in the baseline and test condition did not differ from each other $\left(t_{(5)}=0.712, p=.508\right)$. Also, the end of the learning curve in Part B was significantly higher than the end of the baseline learning curve in PartA $\left(t_{(5)}=-2.583, p=.049\right)$, which explains the interaction between session and experimental Part. This interaction indicates a slightly slower learning rate in the forward interference experiment. The beginning of the learning curve at the $135^{\circ}$ reference in Part B was elevated compared to the end of the learning curve in Part A $\left(t_{(5)}=-12.942, p<.001\right)$. Taken toegther, these results indicate that preceding training at reference orientations at $\pm 30^{\circ}$ from the $135^{\circ}$ reference hampers subsequent training at the $135^{\circ}$ reference orientation.

\section{Comparison of Model and Data}

Model predictions of this scenario are shown in Figure 16A and are similar to the experimental data (Figure 16B). The competition hypothesis predicts interference between training at the $135^{\circ}$ reference and at the $105^{\circ} / 165^{\circ}$ references, irrespective of the order with which these reference orientations are trained. To compare model and behavioral data, difference curves between the baseline training at $135^{\circ}$ and the training that was preceded by training at reference orientations $\pm 30^{\circ}$ from $135^{\circ}$ were calculated for both model and behavioral data (Figure 16C). The fit of the model and empirically observed difference curves between interference and baseline was 

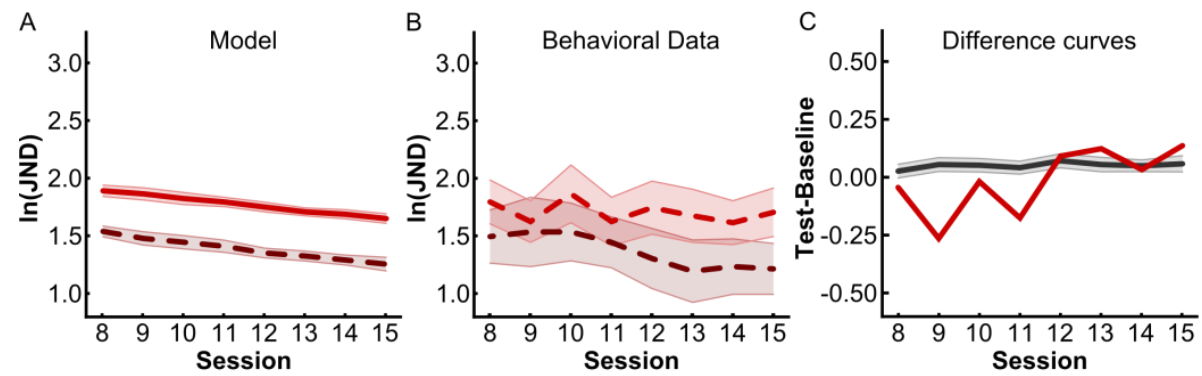

Figure 16. $\boldsymbol{A}$, Modeling data for asymptotic learning (i.e. session 8-15). B, Behavioral data for asymptotic performance of 6 participants. The dashed dark red line belongs to baseline training (Figure 2, Part A, blue/red), whereas the light red line belongs to re-training after intervening training (Figure 2, Part B). $\boldsymbol{C}$, Difference curves between light and dark red line were calculated for modeling data (grey) and behavioral data (red).
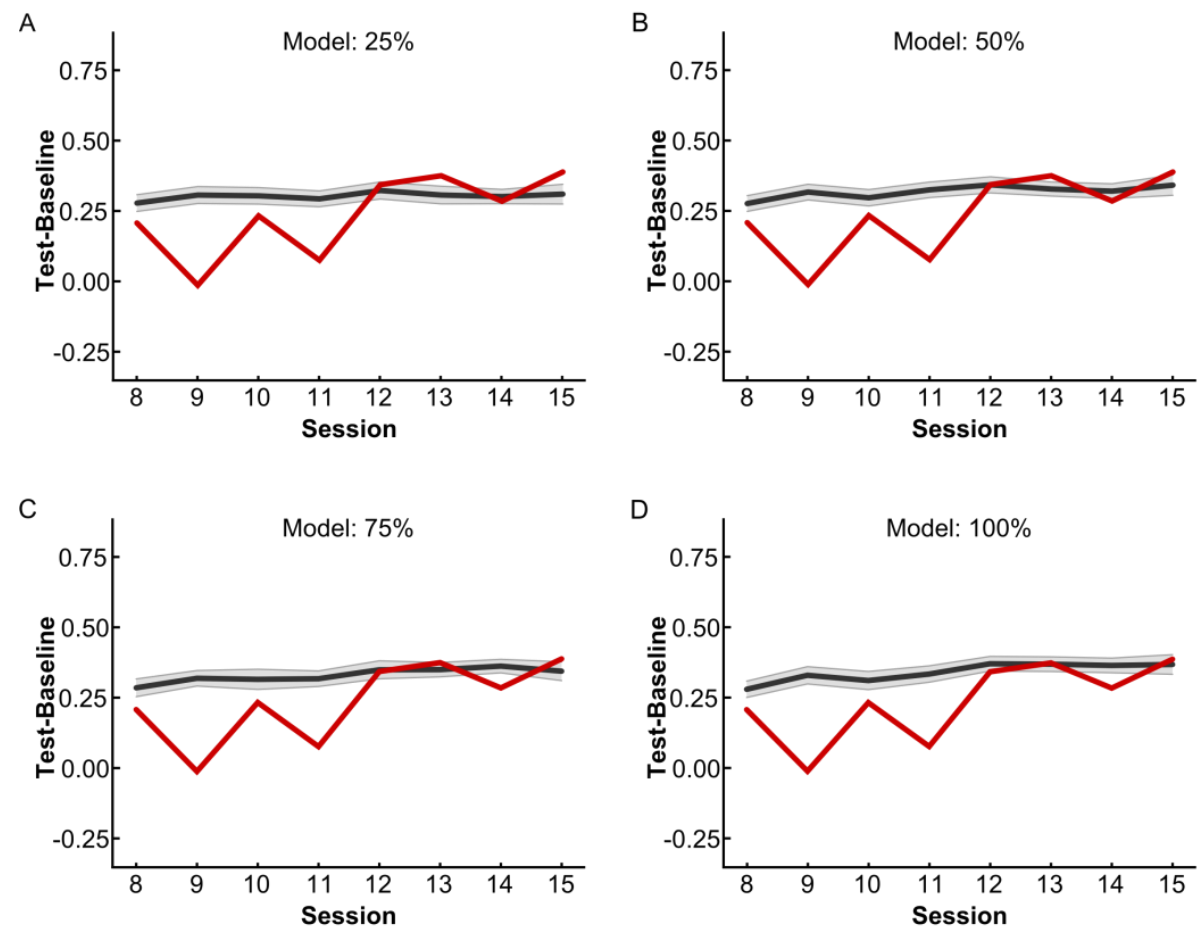

Figure 17. Difference curves for different degree of consolidation: $25 \%(\boldsymbol{A}), 50 \%(\boldsymbol{B}), 75 \%$ $(\boldsymbol{C})$, and $100 \%(\boldsymbol{D})$. Note that all models shown here fare similarly compared to the model shown in Figure 13C with 0\% consolidation. 
significant $(\mathrm{RMSE}=0.15, p=.023)$. Our simple consolidation mechanism which stabilizes a proportion of synaptic changes would also predict interference as it prevents the recruitment of synapses coding for the $135^{\circ}$ reference orientation due to preceding training at the $105^{\circ}$ and $165^{\circ}$ reference orientations. Indeed, as can be seen in Figure 17, predictions of our model when respectively $25 \%, 50 \%, 75 \%$, and $100 \%$ of previously changed synapses undergo consolidation all show a degree of interference similar to our empirical results $(\mathrm{RMSE}=.15, p=.030 ; \mathrm{RMSE}=.15, p$ $=.029 ; \mathrm{RMSE}=.15, p=.023 ; \mathrm{RMSE}=.15, p=.027)$. Adding consolidation does thus not improve upon the explanatory power of the pure competition hypothesis. These results do not imply that the degree of interference did not increase as more synaptic changes are fixed through consolidation. Figure 18 shows that performance levels in the final session progressively decline as consolidation increases. However, it is also apparent that these effects are small and lie well within the range of experimentally observed performance levels. Note that while a more complex consolidation mechanism that would entirely prevent interference can be envisioned, it is not borne out by our data.

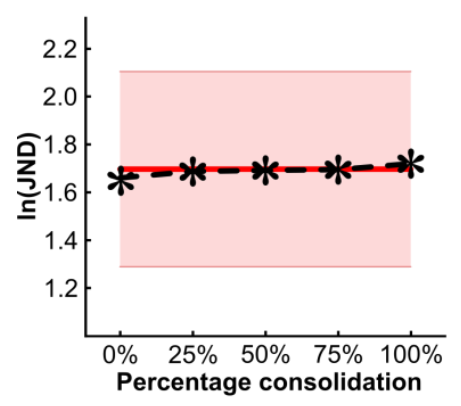

Figure 18. Model performance levels for the final session of Part $\mathrm{C}$ for the red experiment as a function of the percentage of synaptic changes undergoing consolidation (black). Underneath the corresponding mean performance observed empirically (red) is shown with the shaded region representing the SEM. While performance levels of the model decrease with increasing levels of consolidation, all values fall squarely within the shaded region and thus fit the data equally well. 


\section{Comparison of different experimental conditions}

Considering the different conditions in our experiment, it is interesting to compare the performance they elicit at asymptote (i.e. mean over session 8-15). Interestingly, the model predicts an order of performance (on the $135^{\circ}$ orientation discrimination task) which is also present in the data (Figure 19). Performance at asymptote is predicted to be worse after forward interference (FI post, i.e., Part B: red condition), slightly better after backward interference (BI post, i.e. Part C: blue condition), good for naïve training (e.g. BI pre or Ctrl pre, i.e., Part A: blue and green condition respectively), and best for re-training after non-interfering training at an orthogonal reference ( $\mathrm{Ctrl}$ post, i.e., Part C: green condition). Using a repeated measures linear trend analysis, we confirmed that the model prediction of ordered performance

A

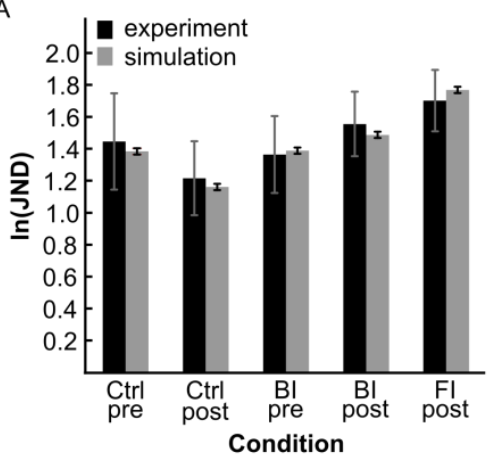

B

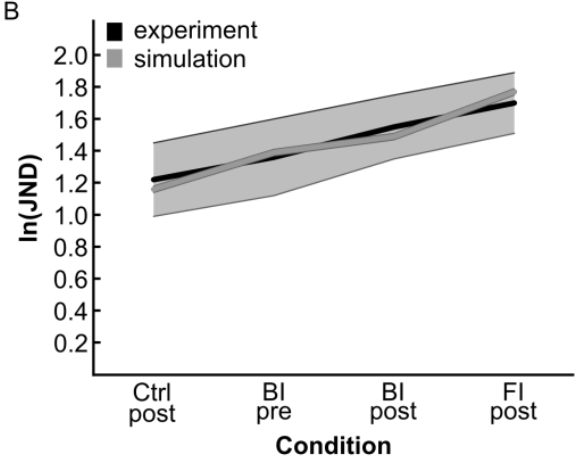

Figure 19. Comparison of performance in the different experiments. A, Mean threshold levels for asymptotic learning (session 8-15) of different conditions before (pre) or after (post) training at other reference orientations (either $45^{\circ}$ or $105^{\circ} / 165^{\circ}$ ). $\boldsymbol{B}$, There is a linear trend seen for training of different conditions. Performance was best after 30 sessions of training with intervening training at an orthogonal reference orientation (double-training: control experiment Part C (Ctrl post)), next comes 15 sessions of initial training (baseline training: Part A for backward interference (BI pre)), followed by re-training after initial training and intervening training at reference orientations differing by $\pm 30^{\circ}$ (Part $\mathrm{C}$ of backward interference experiment (BI post)), and worst is training after training at reference orientations differing by $\pm 30^{\circ}$ without initial training (Part B of forward interference experiment (FI post)). BI: Backward Interference experiment, FI: Forward Interference experiment, Ctrl: Control experiment, pre: before additional training at other reference orientations, post: after additional training at other reference orientations. Shaded areas represent SEM. 
levels is significant $\left(F_{(1,5)}=7.352, p=.042\right)$. Such a trend makes sense because in the forward interference condition (red), training at the $105^{\circ}$ and $165^{\circ}$ references leads to the formation of memory traces that decrease performance at $135^{\circ}$ and subsequent training at $135^{\circ}$ first needs to counteract these effects. In the backward interference condition, initial training at $135^{\circ}$ already softened the effect of interference thus leading to asymptotic performance close to (but still slightly worse than) naïve training at $135^{\circ}$. Thus, when two tasks are competing for the same connections (and with different requirements for those connections) it is the total balance of experience ( $\mathrm{N}$ trials) in the two tasks that will determine how much interference remains from one task upon another. 


\section{Discussion}

Several studies have shown that when two tasks are performed one after the other, the second task can interfere with learned skill for the initially performed task over time intervals ranging from days to weeks (Been et al., 2011; Caithness et al., 2004; Goedert \& Willingham, 2002; Reza Shadmehr \& Brashers-Krug, 1997). These studies also strongly suggested that the strength of interference was associated rather with the extent to which neural populations involved in coding the skill in sensory or motor areas were overlapping (due to task/stimulus similarity), than with the time delay between first and second task. This has led to questions about the basis of behavioral interference, and of skill learning in general. The prolonged time intervals over which interference could be observed, and the importance of representational overlap of memory traces in the tasks as a predictor for behavioral interference, suggests that time-limited consolidation processes are insufficient to understand the properties of memory formation during skill learning and interference. Instead, these properties are better understood when considering processes in which plastic changes underlying memory formation remain malleable. This idea is in line with alternative views on the process of memory formation, both in the domain of hippocampal learning (Forcato et al., 2007; Hupbach et al., 2009; Lewis, 1979; Nadel \& Moscovitch, 2001) and in the domain of skill learning (Walker et al., 2003) in which memory traces can at any time become modified when they are reactivated through new experience. Under that hypothesis, skill memories from different tasks will have to compete for representation if their execution recruits largely a shared pool of neurons.

The aim of the present study was to test the viability of this competition hypothesis, and contrast the competition hypothesis with a consolidation hypothesis, using both psychophysics and modeling. According to the competition hypothesis, behavioral interference will take place when two tasks recruit largely overlapping neural networks, in which skill memory for each task is based on incompatible changes in connection strength in the shared portion of the network. This competition and associated behavioral interference would be expected for any time interval between tasks. According to a slow consolidation process it could be 
expected that interference becomes minimized if two tasks are trained in entirely separate periods of several weeks.

We proceeded to test in human participants whether training at $105^{\circ}$ and $165^{\circ}$ reference orientations for several weeks could destroy earlier acquired skill in orientation discrimination at the $135^{\circ}$ reference, or whether training at $105^{\circ}$ and $165^{\circ}$ references for several weeks could interfere with the later acquisition of new skill in orientation discrimination at the $135^{\circ}$ reference. This contrasts with Been et al. (2011), where the alternation between tasks $\left(135^{\circ}\right.$ vs $\left.105^{\circ} / 165^{\circ}\right)$ occurred at a relatively fast pace (within 0-24h) and led to significant interference (reduced performance at the $135^{\circ}$ reference orientation).

To model learning as well as hypothesized effects of competing tasks on asymptotic performance, we implemented a neuronal network model from Teich and Qian (2003) and enriched it with a dynamic recurrent learning mechanism. Note that with this approach, we focused on low-level changes in sensory areas (such as V1 and V2) that are thought to contribute to asymptotic skill learning and memory formation (Schoups et al., 2001). The recurrent learning mechanism predicted that tuning curves around the trained orientation shift towards that orientation, thus sharpening the tuning curves responsible for discrimination performance at that orientation. As a side effect, tuning curves $\pm 30^{\circ}$ away from the reference orientation are broadened and lead to a decrease in performance at these orientations. This mechanism predicts interference in both a forward and backward interference paradigm. In a forward interference paradigm the tuning curves at the 'original' reference orientation should broaden due to preceding training at the $105^{\circ}$ and $165^{\circ}$ references, and thus new (asymptotic) learning at the $135^{\circ}$ reference first would need to counteract theses adverse effects before any beneficial effects could emerge. Backward interference due to training at the 105 and 165 reference should counteract previously achieved steepening of tuning curves overlapping with the trained $135^{\circ}$ reference orientation. Hence, the competition hypothesis would predict interference in both experiments. In addition, forward interference could be expected to be stronger, as in our design more trials are devoted to the combined training at $105^{\circ}$ and $165^{\circ}$ reference orientations than at the subsequent $135^{\circ}$ reference orientation (red frames in Parts A and B of Figure 2). In contrast, backward 
interference at the $135^{\circ}$ reference (blue frame Part C Figure 2) is tested after a 3 week initial training period at the $135^{\circ}$ reference (blue frame Part A Figure 2), followed by combined training at $105^{\circ}$ and $165^{\circ}$ reference orientations (blue frames Part B Figure 2). Here, the numbers of trials at $135^{\circ}$ versus at $105^{\circ} / 165^{\circ}$ are in balance, so that it can be expected that at the end of learning in the second training period at the $135^{\circ}$ reference, thresholds may approach control levels (i.e. limited interference). In the control experiment, where the potentially interfering reference orientations was removed by $\pm 90^{\circ}$, neuronal populations were expected to be sufficiently segregated, thereby avoiding interference.

The behavioral results at the level of asymptotic learning were in line with these predictions, showing the expected effects for forward and backward interference, as well as anticipated magnitudes in interference in different experimental conditions. Accordingly, interference (simply measured as threshold magnitude) in asymptotic learning of the $135^{\circ}$ condition was largest in the forward interference condition (Experiment 2), slightly smaller for backward interference (Experiment 1), followed by (baseline) training without interference, and smallest in the control experiment in which 2 x 15 sessions were performed separated by noninterfering training (Experiment 1 - Control experiment). These behavioral results closely matched model predictions of interference magnitudes. Moreover, in our simulations we could show that the empirically observed interference effects could not be reproduced with any implementation of a consolidation model, in which different subsets of model synapses were allowed to consolidate.

In our modeling, we considered two types of consolidation models, one in which $100 \%$ of synapses would eventually freeze after completing training, and another type in which a certain proportion of synapses would consolidate, with the rest of the synapses being set by training but remaining malleable. A 'smarter' consolidation process might involve a small subset of connections to freeze upon training, accompanied by a very fast decay of synaptic connections back to baseline for all other connections not undergoing consolidation. This could lead to very sparse memory traces for different skills. Alternatively, improved neuronal segregation might be achieved by a competition process whereby only a fraction of connections change in response to learning in the first place. In these situations, 
more and better segregated memory traces might form in V1 whose consolidation renders them impervious to training on similar tasks/stimuli. Importantly, such a degree of segregation would require equally 'smart' read-out mechanisms whereby neurons higher-up in the hierarchy become increasingly tuned to a single subpopulation in V1. Since we did not implement these read-out mechanisms, however, we did not implement a more efficient consolidation process. Furthermore, our results strongly speak against such 'smart' consolidation as we observe strong interference effects in all conditions. Nonetheless, read-out related changes certainly play a key role in skill learning (Petrov, Dosher, \& Lu, 2005) but rather than tuning extra striate neurons to specific V1 subpopulations which formed during asymptotic learning and which became stable in a consolidation period, they might be the dominant process during early (i.e. non-asymptotic) learning.

In conclusion, our experiments in which tasks were alternately trained over periods of 15 sessions (spread over 3-5 calendar weeks) were designed to favor a slow consolidation process to stabilize memory traces and limit interference. Our empirical observations, in tandem with modeling predictions, argue however against a dominant contribution of consolidation to behavioral interference. Instead, behavioral interference among tasks is likely related to competition among opposite demands on connectivity when the relevant neural population processing the task and stimuli is largely shared (see O’Reilly \& Rudy, 2001; see also Ni \& Maunsell, 2010). The psychophysical data and simulations therefore strongly support a view in which skills that are learned remain permanently malleable. Malleable skill memory traces have high ecological validity in normal skill learning, since reactivation and modification of memory traces is much more efficient than creation of new memory traces for new levels of expertise as would be required in a strict consolidation hypothesis. The price for this flexibility is interference, which may be affordable, as in normal life circumstances it may be unlikely that a single individual would need to learn skills that at the same time compete and are so similar that they can only be memorized in the same network. 


\section{Acknowledgements}

GL was supported by a grant from the Netherlands Organization for Scientific Research to the FPN Graduate School for Cognitive and Clinical Neuroscience (NWO, 022.001.036). PDW was supported by a grant from the Netherlands Organization for Scientific Research (NWO VICI grant, 453.04.002). 


\section{References}

Adini, Y., Sagi, D., \& Tsodyks, M. (2002). Context-enabled learning in the human visual system. Nature, 415(6873), 790-793.

Ahissar, M., \& Hochstein, S. (2004). The reverse hierarchy theory of visual perceptual learning. Trends in Cognitive Sciences, 8(10), 457-464.

Been, M., Jans, B., \& De Weerd, P. (2011). Time-limited consolidation and task interference: No direct link. The Journal of Neuroscience, 31(42), 14944-14951.

Bourtchouladze, R., Abel, T., Berman, N., Gordon, R., Lapidus, K., \& Kandel, E. R. (1998). Different training procedures recruit either one or two critical periods for contextual memory consolidation, each of which requires protein synthesis and pka. Learning and Memory, 5(4), 365-374.

Brashers-Krug, T., Shadmehr, R., \& Bizzi, E. (1996). Consolidation in human motor memory. Nature, 382(6588), 252-255.

Caithness, G., Osu, R., Bays, P., Chase, H., Klassen, J., Kawato, M., . . Flanagan, J. R. (2004). Failure to consolidate the consolidation theory of learning for sensorimotor adaptation tasks. The Journal of Neuroscience, 24(40), 8662-8671.

Casadio, A., Martin, K. C., Giustetto, M., Zhu, H., Chen, M., Bartsch, D., . . . Kandel, E. R. (1999). A transient, neuron-wide form of creb-mediated long-term facilitation can be stabilized at specific synapses by local protein synthesis. Cell, 99(2), 221-237.

De Weerd, P., Pinaud, R., \& Bertini, G. (2006). Plasticity in v1 induced by perceptual learning. New York: Springer Science + Business Media.

Forcato, C., Burgos, V. L., Argibay, P. F., Molina, V. A., Pedreira, M. E., \& Maldonado, H. (2007). Reconsolidation of declarative memory in humans. Learning \& Memory, 14(4), 295-303.

Fuller, S., Rodriguez, R. Z., \& Carrasco, M. (2008). Apparent contrast differs across the vertical meridian: Visual and attentional factors. Journal of Vision, 8(1), 16.

Goedert, K. M., \& Willingham, D. B. (2002). Patterns of interference in sequence learning and prism adaptation inconsistent with the consolidation hypothesis. Learning and Memory, 9(5), 279-292.

Green, D., \& Swets, J. (1966). Signal detection theory and psychophysics. New York: John Wiley, Inc.

Hochstein, S., \& Ahissar, M. (2002). View from the top: Hierarchies and reverse hierarchies in the visual system. Neuron, 36, 791-804. 
Hupbach, A., Gomez, R., \& Nadel, L. (2009). Episodic memory reconsolidation: Updating or source confusion? Memory, 17(5), 502-510.

Karni, A. (1996). The acquisition of perceptual and motor skills: A memory system in the adult human cortex. Cognitive Brain research, 5(1-2), 39-48.

Karni, A., \& Bertini, G. (1997). Learning perceptual skills: Behavioral probes into adult cortical plasticity. Current Opinion in Neurobiology, 7(4), 530-535.

Karni, A., \& Sagi, D. (1993). The time course of learning a visual skill. Nature, 365(6443), 250-252.

Karni, A., Tanne, D., Barton, S. R., Askenasy, J. J. M., \& Sagi, D. (1994). Dependence on rem sleep of overnight improvement of a perceptual skill. Science, 265(5172), 679-682.

Koch, G., Ponzo, V., Di Lorenzo, F., Caltagirone, C., \& Veniero, D. (2013). Hebbian and anti-hebbian spike-timing-dependent plasticity of human corticocortical connections. The Journal of Neuroscience, 33(23), 9725-9733.

Korman, M., Raz, N., Flash, T., \& Karni, A. (2003). Multiple shifts in the representation of a motor sequence during the acquisition of skilled performance. Proceedings of the National Academy of Sciences, 100(21), 12492-12497.

Lehky, S. R., \& Sejnowski, T. J. (1990). Neural model of stereoacuity and depth interpolation based on a distributed representation of stereo disparity. The Journal of Neuroscience, 10(7), 2281-2299.

Lewis, D. J. (1979). Psychobiology of active and inactive memory. Psychological bulletin, 86(5), 1054.

Markram, H., Lübke, J., Frotscher, M., \& Sakmann, B. (1997). Regulation of synaptic efficacy by coincidence of postsynaptic aps and epsps. Science, 275(5297), 213-215.

Montaser-Kouhsari, L., \& Carrasco, M. (2009). Perceptual asymmetries are preserved in short-term memory tasks. Attention, Perception, \& Psychophysics, 71(8), 1782-1792.

Nadel, L., \& Moscovitch, M. (2001). The hippocampal complex and long-term memory revisited. Trends in cognitive sciences, 5(6), 228-230.

Ni, A. M., \& Maunsell, J. H. (2010). Microstimulation reveals limits in detecting different signals from a local cortical region. Current Biology, 20(9), 824-828.

O'Reilly, R. C., \& Rudy, J. W. (2001). Conjunctive representations in learning and memory: Principles of cortical and hippocampal function. Psychological review, $108(2), 311$. 
Peres, R., \& Hochstein, S. (1994). Modeling perceptual learning with multiple interacting elements: A neural network model describing early visual perceptual learning. Journal of computational neuroscience, 1(4), 323-338.

Petrov, A. A., Dosher, B. A., \& Lu, Z.-L. (2005). The dynamics of perceptual learning: An incremental reweighting model. Psychological review, 112(4), 715.

Raiguel, S., Vogels, R., Mysore, S. G., \& Orban, G. A. (2006). Learning to see the difference specifically alters the most informative v4 neurons. The Journal of Neuroscience, 26(24), 6589-6602.

Reymann, K. G., \& Frey, J. U. (2007). The late maintenance of hippocampal ltp: Requirements, phases, 'synaptic tagging', 'late-associativity' and implications. Neuropharmacology, 52(1), 24-40.

Schoups, A. A., Vogels, R., Qian, N., \& Orban, G. A. (2001). Practising orientation identification improves orientation coding in v1 neurons. Nature, 412(6846), 549553.

Seitz, A. R., Yamagishi, N., Werner, B., Goda, N., Kawato, M., \& Watanabe, T. (2005). Task-specific disruption of perceptual learning. Proceedings of the National Academy of Sciences of the United States of America, 102(41), 14895-14900.

Shadlen, M. N., \& Newsome, W. T. (1994). Noise, neural codes and cortical organization. Current opinion in neurobiology, 4(4), 569-579.

Shadmehr, R., \& Brashers-Krug, T. (1997). Functional stages in the formation of human long-term motor memory. The Journal of Neuroscience, 17(1), 409-419.

Shadmehr, R., \& Holcomb, H. H. (1997). Neural correlates of motor memory consolidation. Science, 277(5327), 821-825.

Snowden, R. J., Treue, S., \& Andersen, R. A. (1992). The response of neurons in areas $\mathrm{v} 1$ and $\mathrm{mt}$ of the alert rhesus monkey to moving random dot patterns. Experimental Brain Research, 88(2), 389-400.

Softky, W. R., \& Koch, C. (1993). The highly irregular firing of cortical cells is inconsistent with temporal integration of random epsps. The Journal of Neuroscience, 13(1), 334-350.

Squire, L. R., \& Zola-Morgan, S. (1991). The medial temporal lobe memory system. Science, 253(5026), 1380-1386.

Stickgold, R., James, L., \& Hobson, J. A. (2000). Visual discrimination learning requires sleep after training. Nature Neuroscience, 3(12), 1237-1238.

Talgar, C. P., \& Carrasco, M. (2002). Vertical meridian asymmetry in spatial resolution: Visual and attentional factors. Psychonomic Bulletin \& Review, 9(4), 714-722. 
Teich, A. F., \& Qian, N. (2003). Learning and adaptation in a recurrent model of v1 orientation selectivity. Journal of Neurophysiology, 89(4), 2086-2100.

Teich, A. F., \& Qian, N. (2006). Comparison among some models of orientation selectivity. Journal of Neurophysiology, 96(1), 404-419.

Teich, A. F., \& Qian, N. (2010). V1 orientation plasticity is explained by broadly tuned feedforward inputs and intracortical sharpening. Visual neuroscience, 27(1-2), 57-73.

Walker, M. P., Brakefield, T., Hobson, J. A., \& Stickgold, R. (2003). Dissociable stages of human memory consolidation and reconsolidation. Nature, 425(6958), 616-620.

Wanisch, K., Wotjak, C. T., \& Engelmann, M. (2008). Long-lasting second stage of recognition memory consolidation in mice. Behavioural Brain Research, 186(2), 191-196.

Wetherill, G. B., \& Levitt, H. (1965). Sequential estimation of points on a psychometric function. British Journal of Mathematical and Statistical Psychology, 18(1), 1-10.

Willingham, D. B., Wells, L. A., Farrell, J. M., \& Stemwedel, M. E. (2000). Implicit motor sequence learning is represented in response locations. Memory and Cognition, 28(3), 366-375.

Zhang, J.-Y., Kuai, S.-G., Xiao, L.-Q., Klein, S. A., Levi, D. M., \& Yu, C. (2008). Stimulus coding rules for perceptual learning. PLoS Biology, 6(8), e197. 


\section{CHAPTER 5}

Interference with visual skill learning in humans by exposure to masked, unattended stimuli

Lange, G., Radermacher, A., Jans, B., \& De Weerd, P. (in preparation). Interference with visual skill learning in humans by exposure to masked, unattended stimuli. 


\section{Abstract}

Perceptual learning can take place for stimuli presented outside awareness. We investigated whether stimuli presented outside awareness can interfere with learning. We trained participants in a Gabor orientation discrimination (OD) task at an oblique reference orientation $\left(135^{\circ}\right)$, and tested whether learning could be prevented by additional unattended, masked Gabor stimuli (passive stimulation) around $105^{\circ}$ and $165^{\circ}$ at the trained location. In a previous study, OD training at reference orientations $105^{\circ}$ and $165^{\circ}$ strongly interfered with learning at a $135^{\circ}$ reference (Been, Jans, \& De Weerd, 2011). Here, we report surprisingly strong interference with OD learning at $135^{\circ}$ when additional oriented stimuli around $105^{\circ}$ and $165^{\circ}$ were presented outside awareness. However, interference occurred only when the masked stimulation immediately preceded training, and not when it followed training or when the time gap between masked stimuli and subsequent training was increased to $1 \mathrm{~h}$. We speculate that passive stimulation induces a transient change in network state (adaptation) with a significant capability to interfere with learning, but also with a limited life time. Hence, visual skill in OD at $135^{\circ}$ is comparatively less vulnerable to interference from passive stimulation at $105^{\circ}$ and $165^{\circ}$ than from training at these two orientations (Been et al. 2011). This fits with the idea that skill memory corresponds to a more stabilized network state than that initiated by passive stimulation alone, thereby giving trained stimuli greater potential for interference than passive stimulation. 


\section{Introduction}

A hallmark of memory formation in skill learning is the large improvement between the end of a training session and the start of training on the next day, often referred to as 'latent consolidation' (Karni \& Sagi, 1993; Polat \& Sagi, 1994; Sathian \& Zangaladze, 1997; Schoups, Vogels, \& Orban, 1995). Furthermore, after extensive training, skill performance levels can be stable for periods up to several years (Karni, 1996; Karni \& Sagi, 1993). This is in line with the idea of consolidation leading to stable long-term memories (Hebb, 1949; McGaugh, 1966; G. E. Müller \& Pilzecker, 1900). At the same time, there is much evidence demonstrating interference when training in a task is followed within several hours by training in another, similar task (Brashers-Krug, Shadmehr, \& Bizzi, 1996; Seitz, 2005; Zhang et al., 2008). This indicates that learning/consolidation of the first task is disrupted by the following task (Brashers-Krug et al., 1996; Caithness et al., 2004; Goedert \& Willingham, 2002; Seitz et al., 2005; Shadmehr \& Holcomb, 1997; Zhang et al., 2008). The timing of these interference effects is compatible with the expression window of late genes involved in neuronal plasticity (Luft, Buitrago, Ringer, Dichgans, \& Schulz, 2004), typically expressed up to 6 h after an experience.

However, interference between memory formation for different behavioral tasks has also been reported for much longer time intervals between tasks. For example, Caithness et al. (2004) demonstrated that training in a visuomotor task could be interfered with by a similar task performed several days later. Similarly, Been, Jans, and De Weerd (2011), demonstrated that learning of orientation discrimination of Gabor stimuli at an oblique reference orientation $\left(135^{\circ}\right)$ in a first task was impaired if followed in the same location by a second task in which additional training took place at two other reference orientations tilted $30^{\circ}$ away from oblique $\left(105^{\circ}\right.$ and $\left.165^{\circ}\right)$. The behavioral interference (termed 'active interference', as it results from incompatibilities between actively performed tasks) was present for training intervals up to $24 \mathrm{~h}$, the longest interval tested. The interference depended on the similarity in the orientation of the stimuli used in the first task and in the second task; when the difference was too large $\left(90^{\circ}\right)$, no interference occurred. Taken together, these results indicate that the consolidation of memory traces occurs over a long time (Alvarez \& Squire, 1994), or that memory 
traces remain malleable irrespective of time, and can be reactivated, interfered with, and reconsolidated when a new experience recruits neuronal circuits in which the old memory trace is embedded (Caithness et al., 2004; Nadel \& Land, 2000; Nader, Schafe, \& Le Doux, 2000).

The strong malleability of memory traces raises the question how easy it might be to influence or modify an existing memory trace. Would it be possible to interfere with an existing memory trace by presenting stimuli outside awareness? Although at first sight unlikely, this is a possibility as perceptual skill can be acquired through repeated exposure to stimuli presented outside awareness (e.g., Watanabe, 2001). Mere exposure to stimuli has been shown to both induce adaptive states (Benucci, Saleem, \& Carandini, 2013; Dragoi, Sharma, \& Sur, 2000), and to contribute to perceptual learning (Gutnisky, Hansen, Iliescu, \& Dragoi, 2009; Harris, Gliksberg, \& Sagi, 2012) even when the stimulation occurred outside of awareness (Seitz \& Watanabe, 2005, 2009; Tsushima, Seitz, \& Watanabe, 2008; Watanabe, Náñez, \& Sasaki, 2001). This suggests that the different network states induced by mere exposure to stimuli should have the capacity to interfere with perceptual learning. In the present study, we translated this into the question of whether oriented Gabor stimuli presented outside awareness around $105^{\circ}$ and $165^{\circ}$ would have an interfering effect on Gabor orientation discrimination learning at the $135^{\circ}$ reference, similar to the interference reported when participants performed orientation discrimination on the stimuli around $105^{\circ}$ and $165^{\circ}$ (Been et al., 2011).

The awareness of the Gabor stimuli at $105^{\circ}$ and $165^{\circ}$ reference orientations was limited by presenting them briefly, outside the focus of attention, and masking them. We tested whether these unattended and task-irrelevant 'passive' stimuli were effective in interfering with orientation discrimination learning at the $135^{\circ}$ reference, hence inducing a form of 'passive interference'. Furthermore, we aimed to compare the magnitude of passive interference with the interference documented by Been et al. (2011), which we refer to as 'active interference' because it was obtained during training. Passive stimulation either followed (Experiment 1) or preceded training in the orientation discrimination task (Experiment 2), to compare potential differences between pro- and retroactive interference (Köster, Degel, \& Piper, 2002). To get more insights into the causes for the interference effect and its longevity three more 
experiments were performed: In Experiment 3, we confirmed that the passive stimuli used were indiscriminable and had not been attended. In Experiments 4 and 5, we tested predictions derived from the idea that masked exposure induces a form of adaptation. 


\section{Materials and Methods}

\section{Participants}

In total, 34 naïve participants (26 female, mean age 23.2, SD 4.34) were recruited for this study. All participants had normal or corrected-to-normal visual acuity. After full information about all procedures, informed, written and verbal consent was obtained according to the Helsinki Declaration. Participants were also informed about their right to withdraw participation at any time. All procedures were approved by the local Ethical Committee of the Faculty of Psychology and Neuroscience (ECP). For their participation in the study participants received either monetary reward or credits to fulfill course requirements.

\section{Stimuli, Apparatus, and Task}

Data collection took place in a dimly lit room while participants were sitting in a chair with their head supported by a chin and head rest to keep eye-screen distance constant at $57 \mathrm{~cm}$. Visual stimuli were displayed on a 19" Samsung SyncMaster 940BF LCD monitor (Samsung, Seoul, South Korea; 60Hz refresh rate, 1280x1024 resolution). Eye movements were monitored with a Viewpoint eyetracker v.2.8.3 (Arrington Research, Inc., Scottsdale, Arizona, USA; 60Hz sampling rate). Stimulus presentation and response recording was performed by Cortex v.5.9.6 (NIH freeware for psychophysical and neurophysiological experimentation).

Participants had to perform an orientation discrimination task (Figure 1) in which they had to indicate the direction of the offset from an implicit reference orientation. Each experimental trial started with a time window of $750 \mathrm{~ms}$ during which accurate fixation was to be initiated (i.e., deviation $<1.5^{\circ}$ from fixation dot). Once initiated, fixation had to be maintained for another $250 \mathrm{~ms}$ to trigger stimulus presentation. A Gabor stimulus was shown for $33 \mathrm{~ms}$, and immediately followed by a mask of $49 \mathrm{~ms}$ in conditions in which stimuli were unattended and masked. Stimulus presentation was then followed by a $1467 \mathrm{~ms}$ response window. Responses could be given from the beginning of the stimulus to the end of the response window. They were given with the right middle and index finger, to indicate the clockwise or counterclockwise deviation from the reference orientation, respectively. After each trial, feedback was given to participants by changing the color of the fixation dot for $200 \mathrm{~ms}$ to either 
green or red for correct and incorrect response respectively. After the feedback, the fixation dot disappeared signifying the end of the trial. The intertrial interval (the interval between successive stimulus onsets) depended on the response latencies and the time required for participants to reinitiate fixation. All experiments were done with a gaze-contingent display in which trials only started after $250 \mathrm{~ms}$ of accurate fixation within a $3^{\circ}$ by $3^{\circ}$ window centered on the fixation spot. Trials were aborted as soon as participant's gaze fell outside that window. Aborted trials were repeated at a randomly chosen time during the experiment. The screen was covered by a gray cardboard with an oval aperture so that the screen borders were not visible to participants and thus could not be used as reference for the orientation discrimination task.

Stimuli were Gabor patches (2.37 cycles/degree spatial frequency, 50\% Michelson contrast in the center, $3^{\circ}$ diameter, $33 \mathrm{~ms}$ duration, equiluminant with the $56 \mathrm{~cd} / \mathrm{m}^{2}$ background) presented at small clockwise or counterclockwise deviations from three different reference orientations $\left(105^{\circ}, 135^{\circ}, 165^{\circ}\right)$. In each quadrant, the stimuli were presented at a $6^{\circ}$ eccentricity at equal distances from horizontal and vertical meridians. The fixation dot was a small, white disk $\left(0.2^{\circ}\right.$ diameter $)$. The mask was the same size as the Gabor stimulus, equiluminant with stimuli and
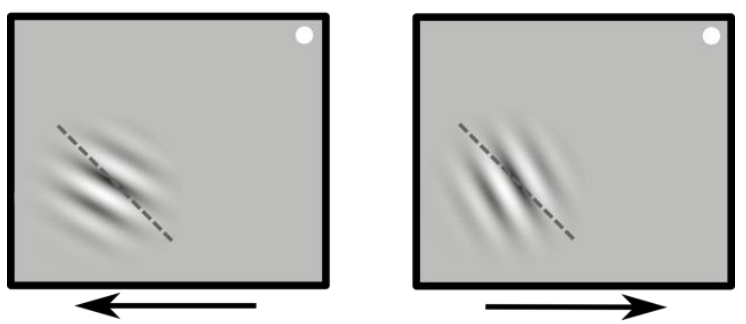

Figure 1. Schematic representation of orientation discrimination task and stimuli. Thresholds were determined using an identification design, with left key responses (left pointing arrow) for orientations deviating counterclockwise from a never-presented $135^{\circ}$ reference orientation, and right key responses (right pointing arrow) for orientations deviating clockwise. Dashed lines are to illustrate the $135^{\circ}$ reference orientation (and were not visible in the stimulus displays). Stimuli are enlarged and only part of the screen is shown for illustration; fixation dot signifies center of display. 
background, and consisted of $0.05^{\circ}$ by $0.05^{\circ}$, randomly positioned pixels of varying shades of gray, white, and black, with a Gaussian filter $\left(\mathrm{HWHM}=0.34^{\circ}\right)$ on top. The timing and spatial characteristics of the mask were piloted in two of the authors. We did not individually calibrate the masks for each subject, as this would have involved presenting the Gabor stimuli prior to the experiment, which would have induced awareness of the Gabor stimuli in the masked conditions, and could have influenced training and interference effects. We deliberately chose to use a nonoptimal spatial frequency for the mask (much higher spatial frequency in the mask than in the Gabor) to avoid completely preventing processing of the passive stimulus (Bruchmann, Breitmeyer, \& Pantev, 2010), as we reasoned a minimum of processing would be necessary to induce a possible interference effect. Moreover, as attention was directed to another quadrant of the visual field during presentation of the masked passive stimuli, we reasoned perfect masking was not necessary to arrive at a significant suppression of awareness of these stimuli. Following the same reasoning, the chosen stimulus termination asynchrony of $49 \mathrm{~ms}$ was also chosen to obtain good but not maximal efficiency, based on data from Macknik and Livingstone (1998).

\section{General features of threshold measurements, design, and data analyses}

We used a Wetherill \& Levitt (1965) staircase tracking 84\% correct performance to acquire just-noticeable differences (JNDs). The staircase ended when either 14 reversal points were acquired or 120 trials were completed, whichever came first. In total, participants completed 16 staircases in each session, and a total of 240 staircases for each complete experiment, except for Experiment 3 and 4 where they only performed 4 staircases per experiment for control purposes (see below). From each staircase, the last ten reversal points were selected to contribute to threshold estimation. Since four staircases were done per quadrant in each session, we determined the threshold per quadrant/session as the geometric mean of 40 reversal points. The start level of each staircase was set to an orientation deviation of approximately $15^{\circ}$ for the first session, and for all subsequent sessions the average threshold level of the previous session was taken as starting level. Orientation deviation was adapted to performance in a proportional manner, by either dividing 
or multiplying the current orientation difference by a factor 1.2 , depending on performance criteria designed for the staircase to converge on a $84 \%$ correct level. All thresholds were ln-transformed.

In the main experiments 1 and 2, masked (passive) stimuli were presented in one of the lower quadrants while orientation discrimination was performed at the $135^{\circ}$ reference in the upper quadrants. The passive stimulation in the two lower quadrants was arranged to create an experimental quadrant where interference with training at $135^{\circ}$ was expected (exposed to stimuli around both $105^{\circ}$ and $165^{\circ}$ ) and a control quadrant where this was not expected (exposed to stimuli around $45^{\circ}$ ). The passive stimulation condition was created during performance of orientation discrimination in the upper quadrants, and the effect of the passive stimulation was tested by additional training in the lower quadrants. Hence, in these experiments, we obtained non-interfered training data not only in the control quadrant but also in the two upper quadrants. Analysis of the two upper quadrants and the control quadrant in Experiment 1 and 2 (including all participants) revealed only a session effect $\left(F_{(14}\right.$, 238) $=44.074, p<.001)$ and no significant main effect of quadrant $\left(F_{(2,34)}=1.927, p\right.$ $=.161)$ and interaction $\left(F_{(8.527,144.951)}=1.880, p=.063\right)$. Therefore, in experiments 1 and 2 (and also 6, which had a similar design), we did not further analyze data from the upper quadrants and only considered experimental and control quadrant data. There is an exception to this in two participants of Experiment 1, in whom the condition with masked exposure to stimuli around the $45^{\circ}$ reference was missing (as another orientation had been masked not useful in the present experiment). When excluding those participants, the performance in the upper quadrants was slightly but significantly worse than in the control quadrant $\left(F_{(2,34)}=3.514, p=.043\right)$. Based on the afore-mentioned statistical analysis, we took thresholds from a single upper quadrant was used as control data in these two participants, if anything that choice would be conservative. Another exception is made in experiment 5, where we also used training data of a single upper quadrant as baseline, because the lower quadrant typically used for control, was used here for an additional experimental condition (see Results for details). The choice between left and right upper quadrant was based on odd and even participant numbers in data acquisition. 
For each learning experiment (Experiment 1, 2, 5, and 6) repeated measures ANOVAs with quadrant (experimental and control) and session as factors were performed on the complete learning curve of fifteen sessions, and separately on the part that encompassed only asymptotic learning (session 7-15). In the case of behavioral interference, analysis including the whole learning curve was expected to reveal an interaction, with the session effect differing per quadrant because of slowed-down learning in the experimental quadrant. Analysis of asymptotic learning then was expected to only reveal a quadrant effect, since interference is thought to start with asymptotic learning (Been et al., 2011). On the data from Experiment 3, a repeated measures ANOVA with stimulus position (upper quadrant, left and right lower quadrants) as factor was performed. Secondly, a repeated measures ANOVA was employed to compare thresholds obtained in Experiment 3 and 4 for the upper quadrant of the three conditions (3-targets, 1-target, and baseline). In cases where the sphericity assumption was violated, Greenhouse-Geisser correction was applied. Pairwise comparisons were adjusted for multiple comparisons using Bonferroni correction.

To determine the starting point of asymptotic learning in the learning experiments, a piecewise nonlinear regression fitting procedure was applied to the $\ln$ transformed threshold of the control condition. Two exponentially decreasing functions were fitted to the data, one to the early part and the other to the asymptotic part of the learning curve. This fitting procedure was applied for all possible transition points between early and asymptotic learning, so that a transition point could be determined for which the total fit was optimized. The starting point and end point of the fit were constrained by taking the mean of the first two sessions and of the last three sessions respectively (for a similar procedure see Been et al., 2011). The inflection point provided by the fitting procedure was at session 6.6 for Experiment 1, at session 5.9 for Experiment 2, at session 6.7 for Experiment 3, and at session 7.0 for Experiment 4. The mean inflection point was at session 6.53, so we took session 7 as starting point for the asymptote in all experiments. This is also in good agreement with the inflection point of 7.3 reported by Been et al. (2011). Therefore, asymptotic learning was determined to include the period from session 7 to 15 (i.e., the last 9 sessions). 
To compare the asymptotic performance of the two passive interference experiments (each $n=9)$ with the active interference experiment $(n=29)$ by Been et al. (2011), we used a bootstrap resampling with random replacement procedure to account for the different participants numbers. The asymptote included mean data from session 7 to 15 in all three experiments. Furthermore, to compare experiments, we had to take into account the differences in the level of thresholds in the control conditions among experiments. To that aim, we subtracted in each experiment the overall across-participants mean of the control condition from each individual threshold in each participant in both control and experimental conditions. This procedure yielded a mean for the control condition of 0 in each experiment, and removed baseline differences among experiments from the thresholds in the experimental data. This normalization was applied after the ln-transformation that we applied by default in all experiments. After the normalization, we drew 1000 times nine participants (to equal $n=9$ in our passive interference experiments) out of the pool of 29 , resulting in a control distribution of normalized thresholds. We then compared the mean asymptotic threshold of the experimental conditions of all three experiments to the distribution of mean thresholds for the control condition.

\section{Experiment 1 \& 2 - Influence of passive exposure on training}

The aim of this experiment was to assess how passive exposure to oriented stimuli influences learning of an orientation discrimination task. In experiment 1, exposure preceded training, and in Experiment 2, exposure followed training. Results are compared with those obtained by Been et al. (2011).

\section{Participants and Procedure}

Nine participants were recruited for Experiment 1 (7 female, mean age 23.3, SD 2.58) and for Experiment 2 (6 female, mean age 23.1, SD 3.28) each. Participants were trained in the orientation discrimination task over a period of 15 sessions. None of the participants had prior experience with visual skill learning experiments.

Each daily session consisted of four conditions (see Figure 2) in each of which participants performed four staircases. The four conditions included two exposure conditions (E45,105 and E45,165) and two training conditions (Tctrl and Texpt). The two exposure conditions had the purpose of presenting different patterns of 
passive exposure to the lower quadrants during dummy training' in the upper quadrants. In the control lower quadrant, passive exposure stimuli were presented around a $45^{\circ}$ reference orientation in all but two participants (in whom statistically equivalent data from a randomly selected upper (dummy) quadrant were used as control data (see General features of threshold measurements, design, and data

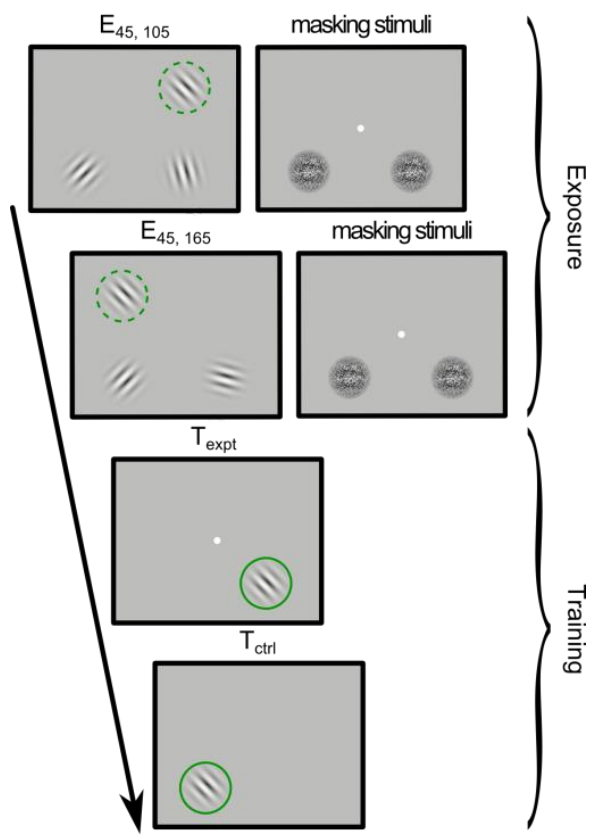

Figure 2. The four conditions of the experiments, two for exposure (E45, 105 and E45, 165), and two for training at the $135^{\circ}$ reference orientation in lower quadrants (Tctrl and Texpt). Passive exposure stimuli in one lower quadrant were presented at around the reference orientation of $105^{\circ}$ or $165^{\circ}$ (in this example right lower quadrant, see E45, 105 and E45, 165), and in the other lower quadrant at around $45^{\circ}$ (in this example left lower quadrant, see E45, 105 and E45, 165). Passive exposure stimuli were presented with small, threshold-level orientation offsets from the references (see Methods). In Experiment 1 (design illustrated here), exposure conditions (E45, 105 and E45, 165) preceded the training conditions (Tctrl and Texpt). In Experiment 2, training conditions (Tctrl and Texpt) preceded exposure conditions (E45, 105 and E45, 165). Green circles were not present in actual stimulus displays. Dashed circles indicate locations of dummy training (used to divert attention away from passive exposure locations, shown without any surrounding circles). Full circles indicate training positions of interest (experimental Texpt and control Tctrl). 
analyses). In the experimental lower quadrant, passive exposure stimuli were presented either around the $105^{\circ}$ or the $165^{\circ}$ reference orientation. When administering training at the $135^{\circ}$ reference orientation in the lower quadrants, either preceding or following passive exposure, interference of training by the passive exposure was expected in the experimental but not the control quadrant. During the two exposure conditions (Figure 2, $\mathrm{E}_{45}, 105$ and $\mathrm{E}_{45}, 165$ ), 'dummy' orientation discrimination training was performed at the $135^{\circ}$ reference orientation in each of the two upper quadrants, while passive stimulation was presented simultaneously in the lower two quadrants. Participants were instructed to ignore any stimulation (i.e., masked Gabor stimuli) flashed in the lower quadrants while performing the task in the upper quadrants. Based on results from Been et al. (2011), the passive stimuli in the experimental lower quadrant were presented around the $105^{\circ}$ and $165^{\circ}$ reference orientations (Figure 2, right lower quadrant in $\mathrm{E}_{45,105}$ and $\mathrm{E}_{45,165)}$. Their proximity in terms of orientation with the $135^{\circ}$ reference orientation was expected to cause interference with training at that reference orientation performed at a different time in the same location (Figure 2, $\mathrm{T}_{\text {expt }}$ ). In the control quadrant (Figure 2, left lower quadrant in $\mathrm{E}_{45}, 105$ and $\left.\mathrm{E}_{45}, 165\right)$, passive stimuli were presented at reference orientations not expected to interfere with orientation discrimination training at the $135^{\circ}$ reference orientation in the same location (Figure $1 \mathrm{~B}, \mathrm{~T}_{\mathrm{ctrl}}$ ). The masked passive control stimuli were used to exclude the possibility that the mere presentation of passive stimuli interfered with learning regardless of orientation, and also to exclude the possibility that the mask itself caused passive interference. The passive stimuli in the lower quadrants had offsets from their respective reference orientations that matched the offsets of the stimuli being discriminated in the upper quadrant on a trial by trial basis. However, the clockwise/counterclockwise deviations from the reference for the actively discriminated stimuli in upper quadrants and passively presented stimuli in lower quadrants were unrelated. Hence, the statistics of stimulation during active discrimination and passive exposure were fully matched, but the orientation of any individual passively presented stimulus was not predictive for the orientation of actively discriminated stimuli in the upper quadrants. 
The left/right position of control and experimental quadrants was randomized over participants. Within participants, the order of the passive exposure to stimuli around the $105^{\circ}$ and $165^{\circ}$ reference orientations within the experimental quadrant was counterbalanced over sessions. The pairing of training in left and right upper quadrant with passive exposure to $105^{\circ}$ and $165^{\circ}$ was also counterbalanced across sessions. Additionally, the order of the training conditions $\left(\mathrm{T}_{\text {ctrl }}\right.$ and $\left.\mathrm{T}_{\text {expt }}\right)$ was counterbalanced, such that each order of the exposure conditions $\left(\mathrm{E}_{45}, 105\right.$ preceding/following $\mathrm{E}_{45},{ }_{165}$ ) was combined with each order of the training conditions $\left(\mathrm{T}_{\text {ctrl }}\right.$ preceding/following $\left.\mathrm{T}_{\text {expt }}\right)$. After finishing the experiments participant completed a rating in which visibility of the masked Gabor stimuli in control quadrant was assessed.

\section{Results Experiment 1 - Exposure preceding training}

In Experiment 1, passive exposure preceded training in both the experimental and control quadrants. Passive exposure resulted in much higher thresholds in the experimental quadrant (Figure 3A, red line) than in the control quadrants (Figure 3A, blue line). This indicates that the passive exposure to Gabor orientations around the $105^{\circ}$ and $165^{\circ}$ axes interfered with training at the $135^{\circ}$ reference orientation. These conclusions were confirmed by two repeated measures ANOVAs on the orientation discrimination thresholds. In the first, we included all sessions, with quadrant (experimental and control) and session (1-15) as within-subject factors. There was a significant main effect of quadrant $\left(F_{(1,8)}=10.840, \mathrm{p}=.011\right)$, session $\left(F_{(14,112)}=7.540, \mathrm{p}<.001\right)$, as well as an interaction between session and quadrant $\left(F_{(14,112)}=2.676, \mathrm{p}=.002\right)$, in line with the slower learning rate in experimental compared to the other quadrants. We then performed a separate analysis only involving the asymptotic data to confirm the significance of the interference effect. The repeated measures ANOVA of the sessions 7-15 revealed a significant main effect of quadrant $\left(F_{(1,8)}=22.750, \mathrm{p}=.001\right)$ (Figure 3B), whereas the main effect of session $\left(F_{(8,64)}=1.543, \mathrm{p}=.160\right)$ and the interaction $\left(F_{(8,64)}=1.082, \mathrm{p}=.387\right)$ were not significant. Hence, the asymptotic data from the $135^{\circ}$ reference orientation confirm a significantly interfering effect of preceding passive exposure around the $105^{\circ}$ and $165^{\circ}$ axes on orientation discrimination learning. In addition, our data 
indicate that the interference effect was specific for the location of the actively presented Gabor stimuli; the interference in the experimental location did not spread to the control location. Moreover, the lack of a difference between dummy learning

A

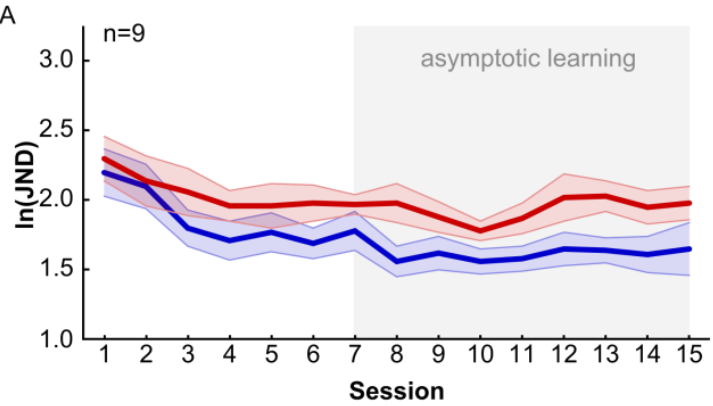

C

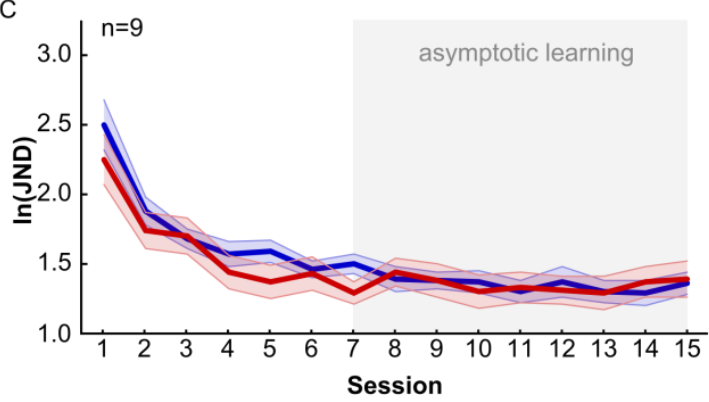

E

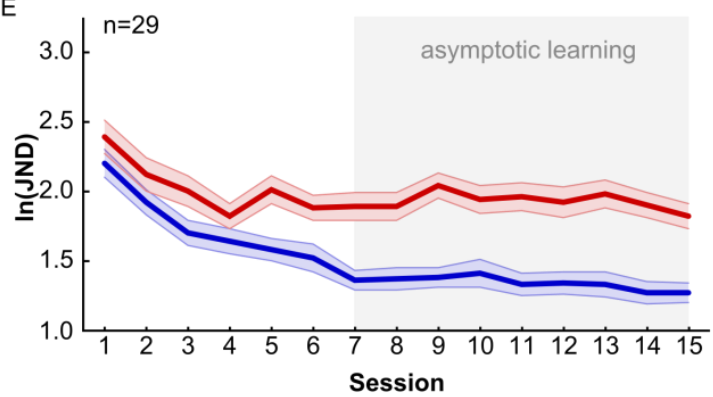

B

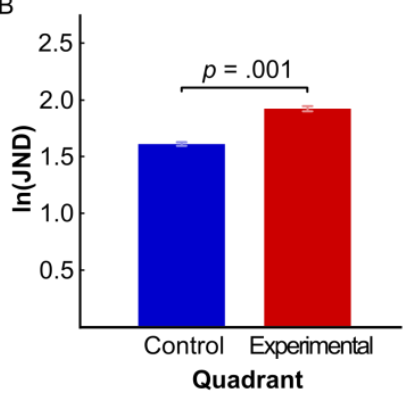

D

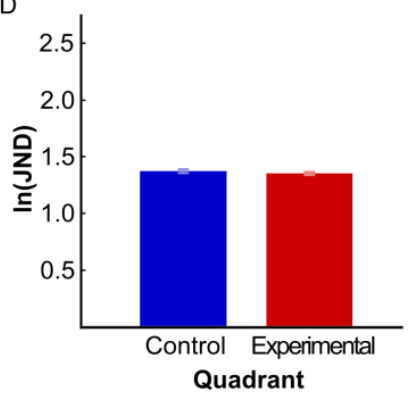

F

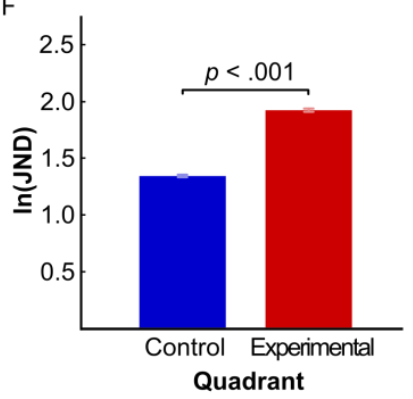

Figure 3. Learning curves and mean performance level during asymptotic learning for experimental and control quadrants. $\boldsymbol{A}, \boldsymbol{C}, \boldsymbol{E}$, Fifteen daily thresholds in experimental quadrant ( $\mathrm{T}_{\text {expt }}$, red line), control quadrant $\left(\mathrm{T}_{\text {ctrl }}\right.$, blue line) plotted as a function of session for Experiment 1 ( $\boldsymbol{A}$, passive exposure preceded training, ), Experiment $2(\boldsymbol{C}$, passive exposure followed training), and from Been et al. (2011) (E, active interference following training). $\boldsymbol{B}, \boldsymbol{D}, \boldsymbol{F}$, Mean performance during asymptotic learning for Experiment $1(\boldsymbol{B})$, Experiment 2 $(\boldsymbol{D})$, and data from Been et al. (2011) $(\boldsymbol{F})$. 
(without preceding passive exposure) and control learning (with preceding passive exposure) demonstrates that the mask alone did not induce interference (see Methods, General features of threshold measurements, design, and data analyses).

\section{Results Experiment 2 - Exposure following training}

In Experiment 2, passive exposure followed immediately after training in both the experimental and control quadrants. The data from this experiment show equivalent decreases of thresholds as a function of daily session in both quadrants (Figure 3C). This was confirmed by two repeated measures ANOVAs. In the first ANOVA the thresholds of all sessions were included. We found a significant main effect of session $\left(F_{(14,112)}=28.426, \mathrm{p}<.001\right)$, but a non-significant main effect of quadrant $\left(F_{(1,8)}=0.5342, \mathrm{p}=.575\right)$ and a significant interaction $\left(F_{(14,112)}=1.986, \mathrm{p}=.025\right)$. Secondly, when analyzing the last nine sessions (asymptotic learning), the quadrant $\operatorname{effect}\left(F_{(1,8)}=0.026, \mathrm{p}=.875\right)$, the session effect $\left(F_{(8,64)}=1.313, \mathrm{p}=.253\right)$, and the interaction $\left(F_{(8,64)}=1.780 \mathrm{p}=.098\right)$ were all not significant (Figure 3D). Furthermore, the learning curves obtained during 'dummy' threshold measurements in the upper quadrants (see Methods, General features of threshold measurements, design, and data analyses) were not different from those measured in the control quadrant. Therefore, passive exposure did not affect learning in neither the experimental nor the control quadrants. Taken together, these results show that passive interference did not take place when exposure to passive stimuli happened after active learning.

\section{Comparison of active and passive interference}

Remarkably, training at the $135^{\circ}$ reference was not interfered with by subsequent passive stimulation at nearby reference orientations. This contrasts with Been et al. (2011) who did show interference when training at the $135^{\circ}$ reference was followed by actively processed stimuli at nearby reference orientations. In the present study, interference was observed only in the condition in which the passive stimulation at $105^{\circ}$ and $165^{\circ}$ reference orientations preceded training at the $135^{\circ}$ reference. We

were interested in comparing the magnitude of the interference effects in this condition with the effect in Been et al. (2011). In the latter study (see their Figure 2) daily training at a $135^{\circ}$ reference orientation by 29 participants in a control quadrant 
was compared with training at a $135^{\circ}$ reference orientation in an experimental quadrant, where additional training was performed in the same location at the $105^{\circ}$ and $165^{\circ}$ reference orientations (referred to as active interference). In each of these four conditions, a block of 4 staircase thresholds was determined in 15 consecutive daily sessions. The comparison of interest for us in Been et al. (2011) is between the learning curves in control and experimental quadrants (Figure 3E). To compare the passive interference effect in Experiment 1 and the lack of passive interference in Experiment 2 with the active interference effect reported by Been et al. (2011), we first normalized the data in control and experimental conditions from our experiments and Been et al.'s (2011) by subtracting within each experiment the overall mean of the control data from all individual thresholds in the entire experiment (see Materials and Methods). This removed the contributions of baseline differences among experiments when comparing the control data in Been et al. (2011) with experimental conditions from our Experiments 1 and 2, and the experimental condition in Been et al. (2011). We then used a bootstrap resampling procedure with random replacement to construct a bootstrapped control distribution of performance without interference. The distribution of control performance was based on 1000 draws of a sample of nine (the sample size used in current experiment) from the population of 29 participants in Been et al (2011). We found that the mean normalized thresholds in the active interference condition in Been et al. (2011) fell outside the bootstrapped control distribution while the passive interference condition in the experiment with passive exposure preceding training (Experiment 1) fell close to edge of the control distribution (red lines in Figure 4, both beyond percentile 99). Hence, both interference effects were strong, although the passive interference effect in Experiment 1 appeared not to reach the same magnitude as in Been et al. (2011). By contrast, the mean of the passive interference condition in the experiment in which exposure followed training (Experiment 2) fell close to the center of the bootstrapped control distribution. In conclusion, when exposure to passive stimuli around the $105^{\circ}$ and $165^{\circ}$ axes preceded training at $135^{\circ}$ reference orientation, the resulting interference with learning at the $135^{\circ}$ reference orientation was surprisingly effective, but not as effective as interference induced by 
active training at the $105^{\circ}$ and $165^{\circ}$ reference orientations. However the interference effect of passive stimuli disappeared with a reversal of the order between exposure and training.

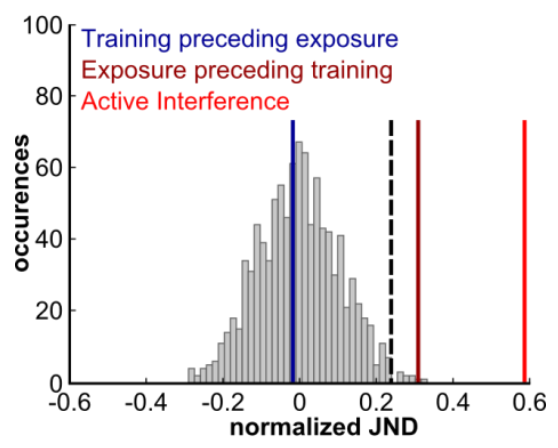

Figure 4. Comparison of the magnitude of interference by training and interference by exposure. Distribution of resampled mean performance levels during asymptotic learning (1000 draws of 9 out of 29 participants) is shown for the control quadrant of the active interference experiment of Been et al. (2011). Superimposed colored lines indicate the mean values for the experimental quadrant for the three different experiments. Blue: Experiment 1, passive exposure preceding training; dark red: Experiment 1, exposure preceding training; light red: interference following training (Been et al., 2011). The dashed line indicates the most extreme $1 \%$ of control condition means.

\section{Experiments 3 and 4 - Control experiments on the discriminability of masked stimuli}

To verify that the passive (masked) stimuli were indiscriminable and had not been attended, all participants performed two control experiments in which they were instructed to attempt discrimination of the masked stimuli. The control experiments were done after the main experiments (1 and 2) for two reasons. First, we did not want to make participants aware of the presence of Gabor stimuli at locations in which the same stimuli were meant to be presented passively later on during the main experiment. Second, the control experiments involved orientation discrimination, which runs counter the purpose of presenting passive stimuli and which might have primed participants to attempt discrimination of the masked stimuli during the main experiment. 


\section{Participants and Procedure}

The 18 participants of Experiment 1 and 2 also completed two control experiments. In the first control experiment (referred to as 3-targets experiment in Figure 5), participants had to discriminate the orientation of not only a target stimulus in the upper quadrant (as done during dummy training in Experiments 1 and 2 to divert attention away from the passive stimuli in lower quadrants), but also of the simultaneously presented masked stimuli in the two lower quadrants (which in experiments 1 and 2 were ignored). The upper quadrant location used in this control experiment was selected at random, and the three targets are referred to as target 1

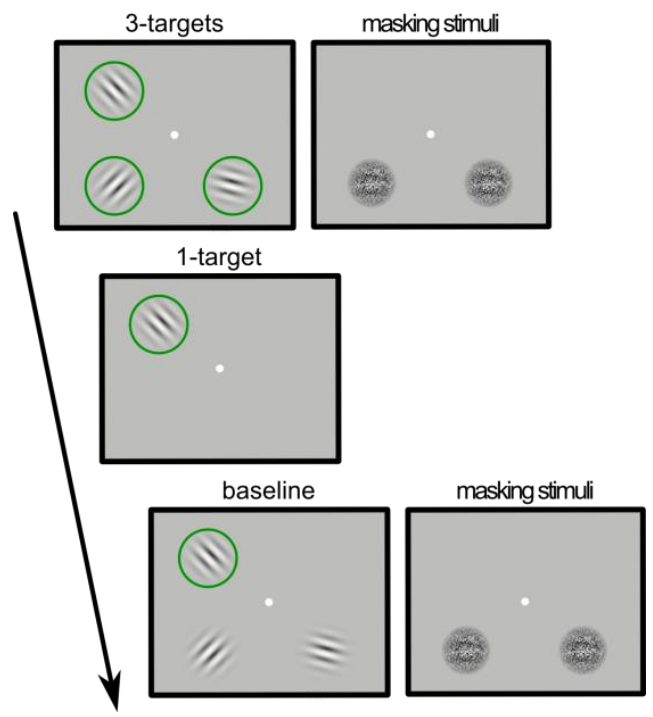

Figure 5. Experimental design for the control experiments. In the 3-targets control experiment (top) participants had to perform the orientation discrimination task on all three positions, including the masked stimuli which had been passive in Experiments 1 and 2. In the main condition of the 1-target experiment (middle) no stimuli were presented in the two lower quadrants. Into the 1-target experiment, we included for analysis baseline data from the last session of Experiments 1 and 2 (bottom), where the task was performed on the stimulus in the upper quadrant while masked stimuli in lower quadrants were ignored. Further, we included the data from the upper quadrant from the 3-targets condition. Green circles indicate stimuli on which orientation discrimination task was performed. Masking stimuli are shown to the right of conditions where they were applied. 
(one of the upper quadrants), target 2 (left lower quadrant), and target 3 (right lower quadrant). Participants in the 3-targets control experiments were instructed to distribute their attention equally to all three stimuli and to respond sequentially in a pre-determined order (target 1 in the randomly picked upper quadrant, target 2 in bottom left quadrant, target 3 in bottom right quadrant). The reference orientations in the three tested quadrants of any given participant were those to which they had been exposed to in the main experiment: In the upper quadrant, this was the $135^{\circ}$ reference orientation, in the experimental quadrant this was either the $105^{\circ}$ or $165^{\circ}$ reference orientation (picked at random in each participant), and in the control quadrant this was the control reference orientation (mostly $45^{\circ}$, see Methods). In a second control experiment, the 1-target experiment, participants performed orientation discrimination at the $135^{\circ}$ reference orientation in the upper visual field quadrant, without the presence of other stimuli in the visual field (Figure 5, referred to as 1-target). For comparison, we included in the analysis of this experiment data from the last session of the main experiment, in which discriminations in the upper quadrant were performed in the presence of to-be-ignored passive stimulation in the two lower quadrants (Figure 5, referred to as baseline), as well as data from the upper quadrant obtained in the 3-targets control experiment. Hence the 1-target control experiment permitted comparisons between Gabor orientation discrimination performed in a single quadrant (1) without masked stimuli, (2) with masked stimuli that were ignored, and (3) with masked stimuli that were turned into extra targets.

\section{Results}

On the data from the 3-targets experiment (Figure 6A), a repeated measures ANOVA with stimulus position (upper quadrant, left and right lower quadrants) as factor showed a significant main effect of stimulus position $\left(F_{(2,18)}=109.859, p<\right.$. 001), with the experimental stimulus in the upper quadrant yielding much lower thresholds than the thresholds for the previously ignored stimuli in the lower quadrants (target 1 vs. target $2: t_{(13)}=-10.901, p<.001$; target 1 vs. target $3: t_{(12)}=-$ $10.711, p<.001)$. The thresholds for the two previously ignored stimuli, however, did not differ significantly (target 2 vs. target $3: t_{(12)}=-1.740, p=.322$; Figure 6A). Note however that the orientation difference during the staircase was limited to a 
maximum of $45^{\circ}$. Staircases measuring orientation discrimination of the masked stimuli in the lower quadrants went up to the ceiling level of $45^{\circ}$, where participants on average performed at random (see percentages in histogram bars). These data show that participants performed randomly when they attempted to discriminate the orientation of the masked stimuli in addition to that of stimuli in one of the upper quadrants.

In the 1-target experiment (Figure 6B), three different conditions were included. In the first and main condition (1-target), participants performed orientation discrimination at the $135^{\circ}$ reference orientation in the upper visual field quadrant, without the presence of other stimuli in the visual field (left inset at top of Figure 6). Performance in the 1-target condition was compared to baseline performance from Experiments 1 or 2 in the same upper quadrant obtained in the
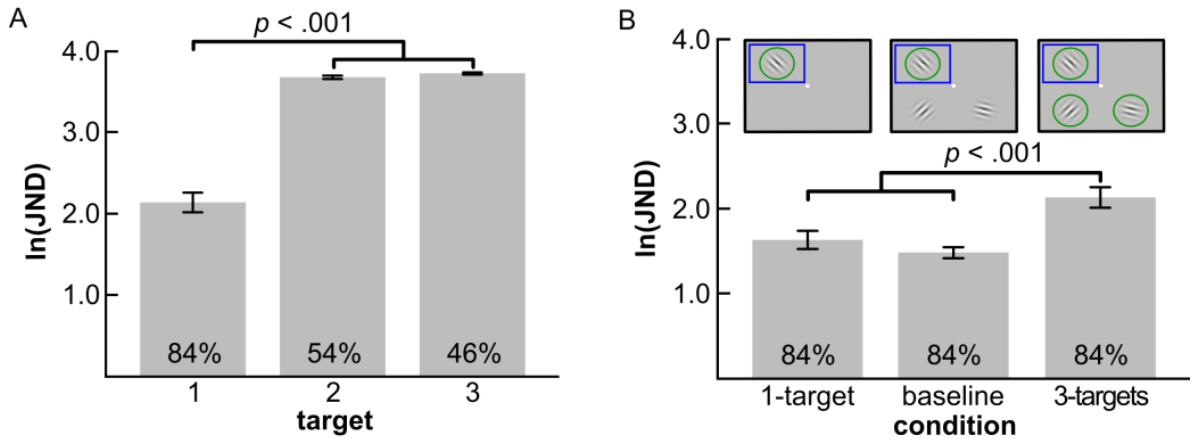

Figure 6. Data from control experiment testing the perception of masked stimuli. $\boldsymbol{A}$, Performance levels of 3-targets condition, in which participants had to perform the task on all three stimuli simultaneously. When in addition to orientation discrimination of original target (target 1), the task was also attempted on masked (formerly ignored) stimuli (targets 2 and 3), no staircase thresholds could be measured for targets 2 and $3\left(\sim 50 \%\right.$ correct at $45^{\circ}$ staircase ceiling). B, Performance levels for the upper quadrant in the two conditions of the control experiment (3-targets and 1-target conditions) was compared to data from the last session of the main experiment (baseline condition). Orientation discrimination was harder in the 3targets condition. Note: target 1 in part A was identical to 3-targets in part B. Green circles indicate the locations at which orientation discrimination task was performed, blue rectangles indicate the quadrant in which performance was compared across conditions, and percentages on histogram bars indicate the percentage correct responses. 
presence of ignored passive stimulation in the lower quadrants (middle inset Figure $6 \mathrm{~B})$. An additional condition included for comparison was performance in the upper quadrant for target 1 in the 3-targets condition (top right inset in Figure 6), obtained while stimulation in the lower quadrants was made task-relevant.

A repeated measures ANOVA was employed to compare normalized thresholds obtained in the upper quadrant selected for each participant in the three conditions of the control experiment shown in Figure 6B. The ANOVA revealed a significant condition effect $\left(F_{(2,34)}=21.500, p<.001\right)$. No difference was observed between the 1 -target and baseline conditions (1-target vs. baseline: $t_{(17)}=1.759, p=.290$ ). Hence, the presence of masked distracters did not have a negative impact on performance in the upper quadrant. However, when the masked stimuli were made task-relevant (3target condition), task performance in the upper quadrant location in the 3-target condition was significantly worse than in the single target condition without masked distracters (3-target vs. 1-target: $t_{(17)}=4.895, p<.001$ ), and also significantly worse when the masked stimuli were task-irrelevant (3-target vs. baseline: $t_{(17)}=5.359, p<$ .001). These results show that an attempt to pay attention

to the masked stimuli increased the dummy thresholds beyond the levels observed in experiments 1 and 2 .

Taken together, these results rule out the possibility that the behavioural interference effects in Experiment 1 were due to actively attending the passive stimuli and/or performing the task in the masked location. On the one hand, active performance of the task on the masked stimuli was shown to be impossible, making the attentional processing of orientation in the stimuli an unlikely driver of any interference effects in the lower quadrants. On the other hand, active performance of the task on the masked stimuli came at the cost of elevated dummy thresholds, which we did not observed in either Experiment 1 or 2 . The fact that attempting to perform the discriminations of the masked stimuli in the lower quadrants negatively impacted performance in the upper quadrant suggests that participants in fact did follow instructions to focus on the discriminations in the upper quadrant while passive stimulation was presented in the lower quadrants.

Further support for the idea that the interference was caused by stimuli that were largely outside of awareness came from introspective reports obtained at the end of 
Experiments 1 and 2. After testing, participants were asked to rate visibility of the masked passive stimuli. Two participants could not describe what they had seen in the lower quadrants, eight participants indicated they had only noticed the mask, five participants indicated they had also noticed on a few individual trials a black and white pattern but without clearly discerning any orientation. One participant reported to have noticed a Gabor stimulus but without having seen its orientation, and two more participants had seen an oriented Gabor stimulus, but also in these individuals orientation discrimination performance of the masked stimuli was at random when they were instructed to discriminate the masked stimuli (highest performance $52.25 \%$ correct). Hence in 10 of 18 participants, there was no awareness of the Gabor stimulus, 6 reported to have occasionally seen a black and white pattern without realizing it had an orientation, and the remaining two participants who reported to have seen orientation could not process it when instructed (as was the case in all other participants). Altogether, this supports the view that in the large majority of trials, there was no awareness of the masked Gabor stimuli.

\section{Experiment 5 - Counteracting interference}

In the condition in which the passively presented stimuli caused interference, this was likely due to a network state that interfered with the memory formation of the actively trained skill in the same location. Exposure leads to adaptation (Buckner et al., 1995; Dale et al., 2000; Demb et al., 1995; Grill-Spector et al., 1999; Henson, Rylands, Ross, Vuilleumeir, \& Rugg, 2004; Henson, Shallice, \& Dolan, 2000; Jiang, Haxby, Martin, Ungerleider, \& Parasuraman, 2000; Li, Miller, \& Desimone, 1993; Miller \& Desimone, 1994; Naccache \& Dehaene, 2001; Sobotka \& Ringo, 1996; Stern et al., 1996; van Turennout, Ellmore, \& Martin, 2000), and our data suggest that adaptation to passive stimuli presented around references $\pm 30^{\circ}$ from the $135^{\circ}$ reference lead to interference during discrimination training at the latter reference orientation. In support of this interpretation, perceptual skill learning has also been linked to adaptation (Gutnisky et al., 2009; Harris et al., 2012), and a recent study showed that skill learning could be interfered with by presenting stimuli tilted away by a $45^{\circ}$ angle from the trained background orientation in a texture detection task (Greenlee \& Magnussen, 1988; Harris et al., 2012). 
Hence, it is possible that a form of adaptation similar to the one induced in Harris et al. (2012) contributed to the effects of passively exposed Gabor stimuli around the $105^{\circ}$ and $165^{\circ}$ references on orientation discrimination at the $135^{\circ}$ reference orientation. Following a suggestion from an anonymous reviewer, we therefore copied the strategy used in Harris et al. (2012) to counteract interference, with the idea of thereby rescuing the learning from the influence of interference. To that aim, we chose in each participant at random one of the two reference orientations used for passive stimulation $\left(105^{\circ}\right.$ or $\left.165^{\circ}\right)$, and added additional passive stimulation tilted $45^{\circ}$ clockwise and counterclockwise from the chosen reference orientation $\left(105^{\circ}\right.$ or $\left.165^{\circ}\right)$. The $45^{\circ}$ difference was based on the study design from Harris et al. (2012), in which adaptation to an oriented texture background was thought to be reduced by presentation of textures oriented $45^{\circ}$ away from the background.

The idea that undoing the effect of just one of the two reference orientations for passive stimulation is sufficient to mitigate the effect of passive interference was based on Been et al. (2011). They found that while performance at the $135^{\circ}$ reference was impaired by additional training at the $105^{\circ}$ and $165^{\circ}$ reference orientations, performance at the $105^{\circ}$ and $165^{\circ}$ reference orientations itself was unaffected. The authors proposed that this was because in lower visual areas involved in orientation discrimination learning, the tuning curve changes that drive improved discrimination at a reference orientation $\mathrm{R}$ (slope increases and decreased sparseness) take place symmetrically around $\mathrm{R}$ in units with preferred orientations $\sim 15^{\circ}$ clockwise and counterclockwise from R (Schoups, Vogels, Qian, \& Orban, 2001; Teich \& Qian, 2003, 2010). To fully counteract improved discrimination at R, it is therefore necessary to counteract these changes in units tuned to orientations clockwise and counterclockwise from $\mathrm{R}$, by training at $\sim 30^{\circ}$ clockwise and counterclockwise from $\mathrm{R}$. When the counteraction occurs only on one side of the trained reference (e.g., clockwise), interference can be expected to be less effective since counteracting the effect of only one of the interfering reference orientations will allow plasticity in a sufficient number of neurons to permit normal performance, and because relatively small numbers of neurons may be sufficient to support normal behavioral performance (Bradley, Skottun, Ohzawa, Sclar, \& Freeman, 
1987; Vogels, 1990). Based on this reasoning, we aimed in Experiment 5 to use the procedure devised by Harris et al. (2012) to counteract the effects of one of the two passive exposure reference orientations $\left(105^{\circ}\right.$ or $\left.165^{\circ}\right)$ to test whether later orientation training at the $135^{\circ}$ reference orientation would show less interference from the passive exposure to stimuli at these $105^{\circ}$ and $165^{\circ}$ reference orientations.

\section{Participants and Procedure}

Eight participants were recruited (6 female, mean age 24.4, SD 7.45) for Experiment 5. As in Experiment 1, dummy training at the $135^{\circ}$ reference orientation was performed in each upper quadrant to present masked, passive stimulation in the lower quadrants. These two blocks of dummy training were followed by two training blocks; one block of training at the $135^{\circ}$ reference orientation in each of the lower quadrants (Figure 7). The passive stimulation in one of the two lower quadrants was limited to a block of stimuli at the $105^{\circ}$ reference orientation and a block at the $165^{\circ}$ reference orientation given during dummy training. For consistency with the previous experiment, we will call this the experimental condition. The passive stimulation in the other quadrant included the $105^{\circ}$ and the $165^{\circ}$ reference orientations as well as passive stimulation aimed to counteract the adaptation at the $105^{\circ}$ or the $165^{\circ}$ reference orientation. This lower quadrant will be called the counteraction condition, in which the training in this quadrant is supposed to be only partly affected by the preceding passive exposure. The passive stimulation in the counteraction quadrant hence required three blocks, i.e. two dummy training blocks as well as a proper training block in the other lower quadrant. The order of the reference orientations of passive stimulation was counterbalanced over sessions in each participant; however, and the counteracting stimulation always directly followed the orientation of which adaptation was to be counteracted. The counteracting stimuli were chosen to be presented around a reference orientation of $60^{\circ}$ (when counteracting adaptation at the $105^{\circ}$ reference) or $30^{\circ}$ (when counteracting adaptation at the $165^{\circ}$ reference). These choices were also motivated by our aim to ensure that the reference orientation for counteracting stimuli differed from the trained $135^{\circ}$ reference orientation by $75^{\circ}$, to ensure they would not by themselves impair learning. The passive exposure reference orientation of which adaptation was to be counteracted was counterbalanced across participants. When 
stimuli were presented at multiple locations (Dummy 1, Dummy 2, $\mathrm{T}_{\text {expt }}$ ), the clockwise versus counterclockwise relation of each orientation to the reference orientation in each location was chosen according to a balanced random string in each location (details in Methods). In addition, for five participants, the orientation difference at the reference orientations showing masked stimuli was set by the

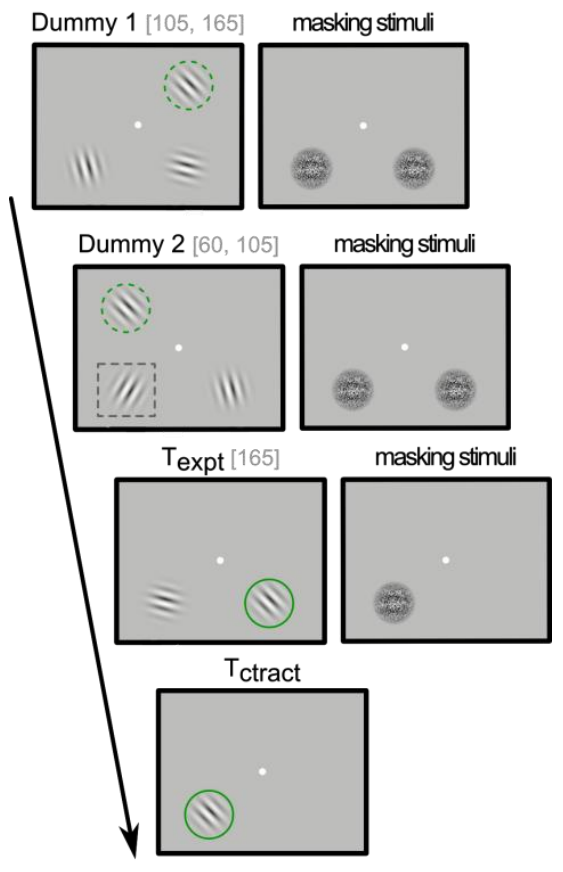

Figure 7. Design for experiment attempting to counteract interference. The design is very similar to that of Experiment 1, except that an additional masked stimulus is shown in one lower quadrant. Both lower quadrants were exposed to stimulation at $105^{\circ}$ and $165^{\circ}$ reference orientations, but in the experimental quadrant, in this example, there was also exposure to stimuli at a $60^{\circ}$ reference orientation (indicated by dashed gray square, not in actual stimulus display), deviating by $45^{\circ}$ from the $105^{\circ}$ reference orientation for passive exposure. Green circles were not present in actual stimulus displays. Dashed green circles indicate locations of dummy training (used to divert attention away from passive exposure locations, shown without any surrounding circles). Full green circles indicate training positions of interest (experimental $\mathrm{T}_{\text {expt }}$ and counteraction $\mathrm{T}_{\text {ctract }}$ ). The gray numbers in brackets behind the condition names indicate reference orientations for passive exposure used in the present example in left and right lower quadrant respectively. 
orientation difference during ongoing training in Dummy 1, Dummy 2, and $\mathrm{T}_{\text {expt }}$ conditions. For three more participants the orientation difference between counterclockwise and clockwise to the average asymptotic performance level in Experiment 1 and 2. This was done to focus passive stimulation around the reference orientations from the beginning of training. In summary, there were three conditions in this experiment. In the baseline condition, we used the average orientation discrimination performance of the two upper quadrants obtained in the absence of masked stimuli. This condition yields orientation thresholds indistinguishable from those obtained with ignored passive stimulation (Experiments 3 and 4). In the standard experimental condition, orientation discrimination was trained with preceding masked stimulation yielding passive interference. In the counteracting condition, the interference from masked stimuli was counteracted by adaptation.

\section{Results}

In the present experiment, we expected that the counteracting-interference condition (with counteracting of passive exposure induced adaptation at either the $105^{\circ}$ or the $165^{\circ}$ reference orientations) might give a level of interference that would be less than in the standard experimental condition (with passive exposure at $105^{\circ}$ and $165^{\circ}$ reference orientations). As a baseline, we used here the orientation threshold obtained in a single upper quadrant where no passive exposure was given at all (for details see General features of threshold measurements, design, and data analyses). Figure 8 shows that our attempt to counteract interference was not successful, with data in the 'counteracting interference' condition (green line) fully overlapping with the data in the standard interference condition. A repeated measures ANOVA with quadrant (upper, counteraction, experimental) and session (1-15) as within-subjects factors revealed a significant main effect of quadrant $\left(F_{(2,14)}=6.734, p=.009\right)$, main effect of session $\left(F_{(14,98)}=18.330, p<.001\right)$ and interaction $\left(F_{(28,196)}=1.635, p=\right.$ .029) (Figure 8). We then performed a separate analysis only involving asymptotic data. A repeated measures ANOVA of sessions 7-15 revealed a significant main effect of quadrant $\left(F_{(2,14)}=7.411, p=.006\right)$ and a non- significant main effect of session $\left(F_{(8,56)}=0.728, p=.667\right)$ and a non-significant interaction $\left(F_{(16,112)}=1.378\right.$, 


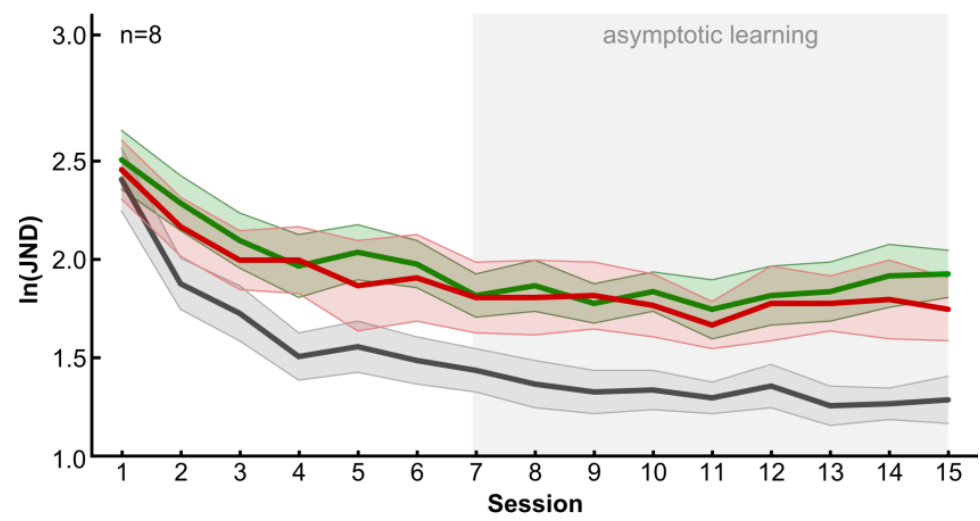

Figure 8. Data from Experiment 5 - counteracting adaptation. No difference was found between the counteraction quadrant (green) where we attempted to counteract adaptation, and the experimental quadrant (red), where exposure preceded training just as in Experiment 1.

$p=.166)$. Pairwise comparisons revealed a significant difference between upper and counteraction quadrant $\left(t_{(7)}=3.912, p=.017\right)$ and upper and experimental quadrant $\left(t_{(7)}=.999\right)$. This analysis confirmed that the procedure we followed to counteract adaptation was not effective in our experiment.

Nevertheless, in the eight participants of the present experiment, we again confirmed a clear interference effect of passive exposure to orientations at $105^{\circ}$ and $165^{\circ}$ on subsequent learning at $135^{\circ}$ (red data plot in Figure 8). Hence, in total, all 17 participants consistently showed an interference effect when passive exposure at references $105^{\circ}$ and $165^{\circ}$ preceded training at $135^{\circ}$. To summarize the effect of passive exposure preceding training in experiments 1 and 5 combined, we had to take into account that in experiment 5 the counteraction condition was different from that used in experiment 1 . Hence, in the summary figure (Figure 9) we pooled all data in the passive exposure condition over all 17 participants from the two experiments combined, and contrasted these data with thresholds from a single upper quadrant (for details see General features of threshold measurements, design, and data analyses); data that was available in both experiments. A repeated measure ANOVA on the pooled data from experiments 1 and 5 with quadrant (upper, experimental) and session (1-15) as within-subjects factors revealed a significant 


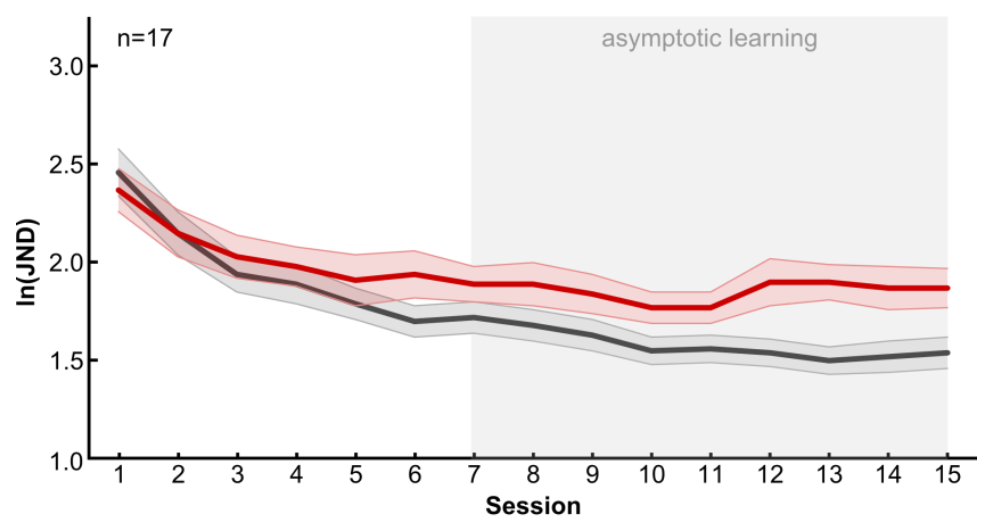

Figure 9. Data from Experiment 1 and 5 combined. Since the control condition in the two experiments differed we used one of the upper quadrants as baseline to compare to the experimental condition. Which of the upper quadrant was chosen was based on odd and even participant numbers in data acquisition.

main effect of quadrant $\left(F_{(1,16)}=36.621, p<.001\right)$, main effect of session $\left(F_{(14,224)}=\right.$ $25.605, p<.001)$ and interaction $\left(F_{(14,224)}=6.083, p<.001\right)$. We then performed a separate analysis only involving the asymptotic data. The repeated measures ANOVA of sessions 7-15 revealed a significant main effect of quadrant $\left(F_{(1,16)}=\right.$ 53.363, $p<.001)$ and non-significant main effect of session $\left(F_{(8,128)}=1.794, p=\right.$ $.084)$ and interaction $\left(F_{(4.659,74.548)}=1.631, p=.167\right)$. The combined data thus clearly show that passive exposure before training leads to interference.

\section{Experiment 6 - Exposure preceding training by 1 hour}

To get more insight into the nature of the underlying mechanism of passive interference, we tested the longevity of the exposure-induced interference effect by introducing a one-hour break between exposure and training. As Been et al. (2011) have shown, training on $135^{\circ}$ can be impaired by subsequent training on $105^{\circ}$ and $165^{\circ}$ in the same quadrant. This effect was shown for time delays between training periods of $0,0.5,1,3,6$, and 24 hours. Here, we aimed to perform an analogous experiment for the interference observed when passive exposure preceded training. To that aim, we introduced a time delay of one hour between passive exposure and 
training. We anticipated that, should the effect of exposure be due to an adaptationlike mechanism, the time delay might reduce or even eliminate the interference effect.

\section{Participants and Procedure}

Eight participants (7 female, mean age 21.9, SD 3.12) were recruited for Experiment 4. The procedure was identical with that of Experiment 1, with the only difference that between exposure and training a 1-hour break was introduced.

\section{Results}

Figure 10A shows the results in the total pool of participants. Although there still appeared to be a trend towards interference, this trend was no longer statistically significant. This was confirmed by a repeated measures ANOVA with quadrant (control, experimental) and session (1-15) as within-subjects factors. This analysis revealed a significant main effect of session $\left(F_{(14,98)}=23.590, p<.001\right)$, but a nonsignificant main effect of quadrant $\left(F_{(1,7)}=2.728, p=.113\right)$ and a non-significant interaction $\left(F_{(14,98)}=0.813, p=.653\right)$. The trend towards interference in the total sample reflected the fact that two out of eight participants did show an interference effect, whereas the remaining six participants showed none (Figure 10B). So, although there was individual variation in the persistence of passive exposure induced interference it seems that the interference effect caused by exposure to oriented stimuli is more transient than the interference effect caused by training (Been et al., 2011), which has been shown to last for at least 24h. This suggests different underlying mechanisms for the two interference effects, and the possibility for contributions of an adaptation-like mechanism in the interference caused by passive exposure. 

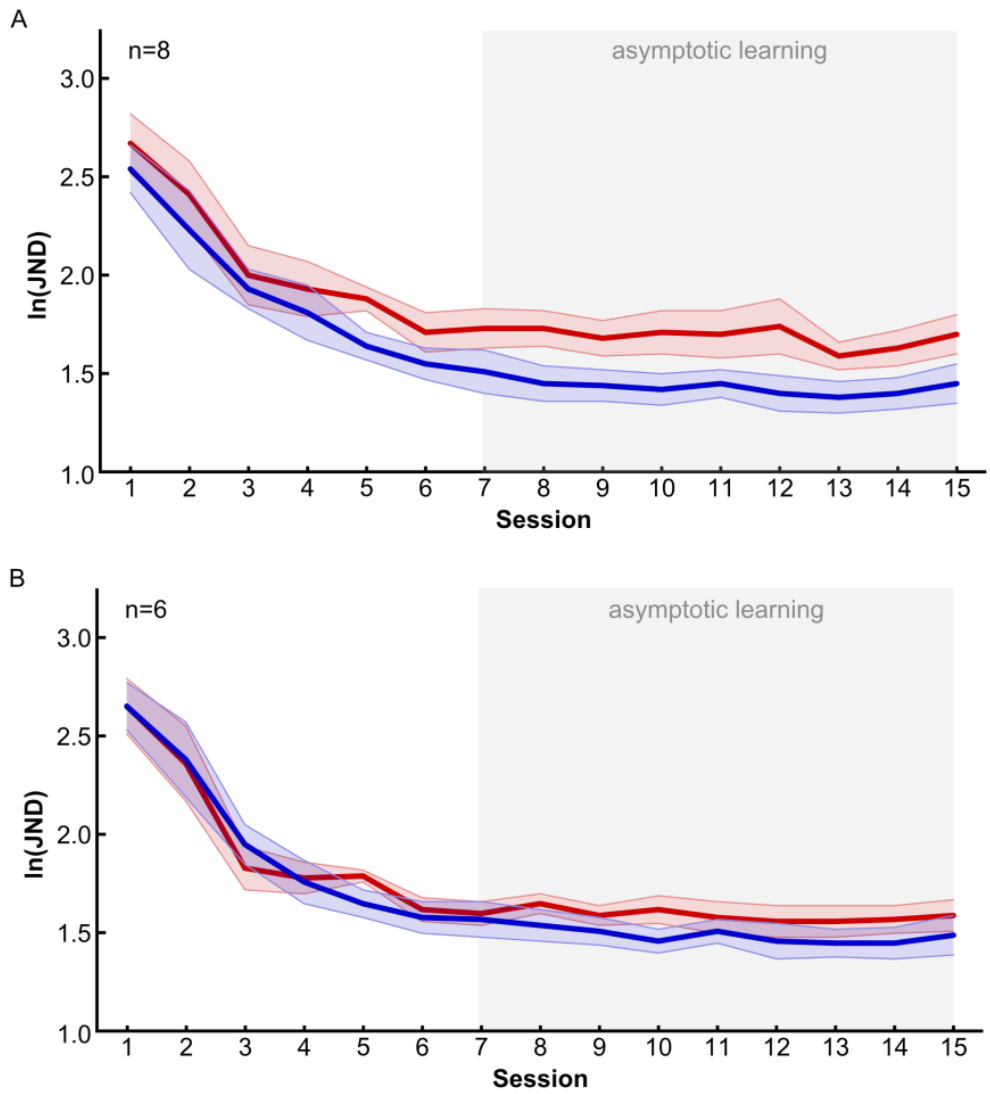

Figure 10. Data from Experiment 6 - Exposure preceding training by 1 hour. A, Although the data seems to indicate a difference between experimental (red) and control quadrant (blue), statistical analyses do not confirm this. $\boldsymbol{B}$, When excluding the two participants who show a clear interference effect in their individual data, the effect was no longer visible in the group data. 


\section{Discussion}

We have investigated whether exposure to masked, unattended stimuli, referred to as 'passive stimulation' can interfere with perceptual learning. To that aim, we used a Gabor orientation discrimination task at a 'trained' reference orientation of $135^{\circ}$, which was immediately followed or preceded by unattended, masked Gabors around two additional reference orientations $105^{\circ}$ and $165^{\circ}$. A control experiment confirmed that in the passive stimulation conditions, participants did not attend the masked Gabors, had no or little awareness of them, and could not discriminate their (masked) orientation when asked. Our experiments revealed that interference by passive stimulation was effective only when passive stimulation immediately preceded training. The interference effect observed under those conditions was sizeable, and approached the magnitude of interference induced by orientation discrimination training at the $105^{\circ}$ and $165^{\circ}$ references upon the preceding orientation discrimination learning at a $135^{\circ}$ reference in Been et al. (2011). However, whereas Been et al. (2011) reported interference when training at the $135^{\circ}$ reference was followed by an additional training task, here, the interference with training at $135^{\circ}$ was observed only when the passive exposure preceded training (Experiment 1 and 2). This was a first indication that the mechanisms of interference are not identical in the two studies. A second indication came from our finding that interference by passive stimulation on subsequent training in most participants could be significantly reduced or abolished by increasing the time delay between passive stimulation and training to 1 hour (Experiment 6). This contrasts with the study of Been et al. (2011), where interference was independent of the time delay between the first and second training episode. This suggests that in the present study, passive exposure induced a network change of limited duration, and with time-limited capacity to interfere with subsequent skill acquisition. Hence, both the timedependency of effects of passive stimulation, as well as the lack of interference when passive stimulation followed training suggest significant differences between the mechanisms underlying interference by a trained task (active interference) and interference by masked, unattended stimuli (passive interference). 
In a model of Teich and Qian (Teich \& Qian, 2003, 2010), a potentially related distinction has been made between the effect of exposure to an oriented stimulus when it is relevant in the context of orientation discrimination training and when the oriented stimulus is simply shown for a prolonged time (mere exposure) in a classical adaptation paradigm. Teich and Qian (2003) have modeled the neuronal tuning changes resulting from training, as reported in Schoups et al. (2001), by a reduction in excitatory connectivity among neurons well-stimulated by the trained orientation. By contrast, the effect of prolonged exposure (during adaptation, without a task) was modeled by a reduction in both excitatory and inhibitory connection input. The latter mechanism may also be modeled by hyperpolarization (see Kohn, 2007). Accordingly, the population states in the training and mere exposure conditions were different. The trained state showed a sharpening of tuning curves, decreased sparseness, and steeper slopes of flanks of tuning curves in neurons tuned about $15^{\circ}$ away from the trained reference (see also Schoups et al., 2001), whereas neurons tuned to orientations far away from the trained reference showed opposite effects (broadened tuning curves, shallower tuning curve flanks, and sparser coding). On the other hand, irrelevant exposure (as in a more typical adaptation paradigm) lead to broadened tuning curves, shallower tuning curve slopes and sparse coding at and around the stimulated orientation, while for fartherremoved orientations the opposite occurred (narrower tuning curves, steeper tuning curve flanks, and decreased sparseness in coding).

To understand the interference effects observed in our study, it is useful to first consider the effects of passive exposure in units (closely) tuned to the reference orientations at which the masked Gabors were presented $\left(105^{\circ}\right.$ and $\left.165^{\circ}\right)$. One could speculate that the exposure to masked, unattended stimuli may create a population state that is more similar to the state obtained after mere, prolonged exposure than to the state obtained after training. In both anesthetized cats (Dragoi et al., 2000) and awake monkeys (Dragoi, Sharma, Miller, \& Sur, 2002), irrelevant prolonged exposure to an oriented stimulus, in a typically simple adaptation paradigm, led to broadened tuning curves and sparser coding. From this network state, impaired orientation discrimination at the 'adapted' reference orientation could be predicted. However, behavioral studies have not consistently supported this, neither in typical 
adaptation paradigms (Regan \& Beverley, 1983); nor in designs using mere exposure (Gutnisky et al., 2009) or subliminal repeated exposure (Nishina, Seitz, Kawato, \& Watanabe, 2007; Watanabe et al., 2001). In the latter three studies, training after repeated exposure was facilitated. Hence, it is possible that the sensory network state induced by passive stimulation in our study created a state more similar to that induced by training, than to that induced by typical adaptation paradigms. Passive stimulation and training induced network states may thus primarily differ in their stability over time, and this might be a crucial factor in understanding the order-effect of passive interference, and its time-limited nature.

Secondly, it is useful to consider what the remote effects are of passive exposure to orientations around $105^{\circ}$ and $165^{\circ}$ upon units tuned to $135^{\circ}$, and how these remote effects may affect orientation discrimination at the $135^{\circ}$ reference. In principle, the Teich and Qian model (2003) predicts that the changes in 'local' network state involving units stimulated well by the exposed orientations affect the 'remote' network involving units not well stimulated by the stimulation, in an opposite manner. If we start from the assumption that passive exposure to an orientation creates a network state similar to that achieved by orientation discrimination training, then passive exposure around $105^{\circ}$ and $165^{\circ}$ will lead to changes in neural tuning detrimental for orientation discrimination in units tuned to orientations $\pm 15^{\circ}$ away from $105^{\circ}$ and $165^{\circ}$ (in the V1-inspired model of Teich and Qian). Training at a $135^{\circ}$ reference orientation therefore is expected to be negatively affected. Based on existing literature of local effects of passive exposure (Watanabe et al., 2001; Nishina et al., 2007; Gutnisky et al., 2009) the predicted remote effect is plausible. Importantly, we cannot exclude the possibility that the passive exposure manipulation at $105^{\circ}$ and $165^{\circ}$ induced a local state detrimental for discrimination, with remote effects facilitating discrimination. However, in the adaptation scenario of Teich and Qian (2003), detrimental effects for discrimination resulting from traditional adaptation spread over an orientation range of at least $\pm 30^{\circ}$ around the exposed reference orientations, with the effects favoring discrimination occurring beyond $\pm 30^{\circ}$. Hence, in the context of a simple V1 model, (Teich and Qian, 2003) irrespective of the specific network state induced by passive exposure around $105^{\circ}$ 
and $165^{\circ}$, the remote network state involving neurons tuned (closely) to the trained reference orientation of $135^{\circ}$ predicts interference. Note however that Teich and Qian's (2003) model was based on V1 orientation selectivity and obviously there are limitations in linking orientation learning and interference with a model of only a single low level area. In addition, even considering just a single area, the model's behavior was dependent on a large set of area specific parameters.

The above considerations give perspective to the statement that adaptation is an important component of skill learning (Goldstone, 1998; Harris et al., 2012; Shadmehr \& Mussa-Ivaldi, 1994). In visual skill learning with oriented stimuli, it has been suggested that exposure to stimuli at a particular orientation induces a type of neural adaptation effect (Dragoi et al., 2000; Felsen et al., 2002; J. R. Müller, Metha, Krauskopf, \& Lennie, 1999) that may constitute a short-term memory trace underlying both behavioral (Gutnisky \& Dragoi, 2008; Gutnisky et al., 2009; Harris et al., 2012) and neurophysiological correlates (Schoups et al., 2001) of skill learning with oriented visual stimuli. Since this is a form of adaptation that also permits increased discrimination, the hypothesized form of adaptation here should be akin to the population state described in Teich and Qian (2003) as resulting from training. Hence, it can be hypothesized that the network effects of passive exposure form the basis of more persistent memory traces when combined with attention and feedback/reward, during training. However, the sensory state induced by passive exposure presented alone, outside the context of a task may be time-limited and less robust. This in turn suggests that interference from passive exposure to stimuli presented $\pm 30^{\circ}$ from a reference orientation will also be time-limited and less robust.

The idea of a time-limited adaptation-like effect driving passive interference is supported by our finding that a time gap between passive exposure at $105^{\circ}$ and $165^{\circ}$ and following training at the $135^{\circ}$ reference orientation substantially reduced the effect of passive interference on orientation discrimination learning. This contrasts with the interference effect reported by Been et al. (2011), who observed interference for a time-interval of up to 24 hours between training periods, whereas our behavioral interference effect induced by passive exposure was absent after a 
one-hour delay in most participants. This suggests a lower weight and persistence for memory traces resulting from passive-exposure-based adaptation than for memory traces formed by pairing adaptation effects with attention and other taskrelated factors during orientation discrimination.

In addition, a time-limited adaptation-like effect driving passive interference might also contribute to the order effect in passive interference. When passive stimulation is presented first, the accumulated network state may carry enough weight to counteract the orientation discrimination task. However, when passive stimulation is presented after training, the resulting network state may fail to exert a delayed effect on training the next day as it may dissipate rapidly over time. In addition, a retroactive effect may also be unlikely, as at the end of training a robust network state may have formed that may be difficult to modify by the slow accumulation of passive stimulation effects. In addition, ongoing interactions with higher-level brain regions involved in attention and reward may help to stabilize the sensory network state after training, rendering it more resistant to interference (De Weerd et al., 2012; Lewis, Baldassarre, Committeri, Romani, \& Corbetta, 2009; Tambini, Ketz, \& Davachi, 2010). This stabilizing influence from other brain regions is likely absent for sensory network states during passive exposure. This additional factor, enhancing the difference in stability over time between the two network states, may also contribute to the order effect in passive interference in the present experiments.

In experiment 5, we aimed to counteract adaptation by presenting a stimulus differing by $45^{\circ}$ from one of the exposure orientations (either $105^{\circ}$ or $165^{\circ}$ ) similarly to Harris et al. (2012). The hypothesis was that by counteracting adaptation of one exposure orientation the interference effect would be eliminated. However, exposure to an additional orientation differing by $45^{\circ}$ from one exposure orientation was not successful in eliminating the interference effect. One possible explanation is that, in contrast to other studies (Greenlee \& Magnussen, 1988; Harris et al., 2012) we did not interleave trials, but only had consecutive blocks with the two orientations. This might not have been as efficient as interleaving trials in counteracting adaptation. Alternatively, the adaptation at one of the passive exposure reference orientations might have been successfully counteracted by our 
manipulation but this might have been insufficient to eliminate the interference effect. Furthermore, it should be noted that the paradigm in the present study, where adaptation was counteracted in the location of the target stimulus, was very different from that in Harris et al (2012), where adaptation was counteracted in a background texture providing a context against which the target stimuli were defined. Finally, although we were led by the $45^{\circ}$ difference between trained and interfering orientations in Harris et al., (2012), according to the model of Teich and Qian (2003) this orientation difference may have been slightly too large to expect the counteracting effects we had aimed for. In the model, adaptation is achieved by reducing both excitation and inhibition. This reduces the overall response amplitude of neurons at the trained orientation and it broadens the tuning curve of neurons thus making it more difficult for them to distinguish between orientations around the adapted orientation. The counteraction effect is likely dictated by the shape of the Mexican hat profile of lateral excitation and inhibition. Since excitation does not reach beyond $45^{\circ}$, adaptation at $60^{\circ}$ (or $30^{\circ}$ ) and ensuing reduction of excitation and inhibition would effectively only reduce inhibition of the orientations presented around $105^{\circ}$ (or $165^{\circ}$ ). The resulting increase of responses to stimuli around $105^{\circ}$ (or $165^{\circ}$ ), if anything, may increase their interfering effect on orientation discrimination learning at $135^{\circ}$ rather than reduce it. Overall, the negative result in experiment 5 is difficult to interpret, and we suggest that the data from experiment 6 still suggest that a slow form of adaptation with a decay time for most participants of less than an hour remains a parsimonious mechanism to explain the effects of passive exposure in the present study.

Our experiments suggest that visual stimuli presented outside awareness and without attention can interfere with the acquisition of visual skills (passive exposure before training), but not so much with an existing skill-level or with consolidation. This indicates that existing visual skill memory traces are not so vulnerable that they would be easily interfered with by irrelevant stimuli presented outside awareness. However, the effectiveness of interfering stimuli when presented prior to training was strong, although transient, being gone in most participants after 1 hour. This suggests that passive interference in our paradigm was driven by an adaptation-like effect, which induced a remote network state (i.e., in orientation-tuned neurons other 
than those stimulated directly by the passive stimulation) that counteracted effective orientation discrimination, and which dissipated over about 1 hour. Previous visual skill learning studies have shown that passive exposure to stimuli in a number of paradigms can contribute to visual skill learning (Watanabe et al., 2001; Nishina et al., 2007; Gutnisky et al., 2009), and it has been suggested that the underlying reason in part may reside in the formation of an adaptation state beneficial for subsequent learning (Gutnisky et al., 2009). The present study provides the first evidence that passive exposure to stimuli similar, but not identical, to those used for training, can also interfere with visual skill acquisition. We suggest that this interference effect is driven by remote effects of the same form of adaptation that in other paradigms can facilitate visual skill learning. 


\section{Acknowledgements}

The research was supported by the FPN Graduate School for Cognitive and Clinical Neuroscience (NWO, 022.001.036), and by a grant from the Netherlands Organization for Scientific Research to PDW (NWO VICI grant, 453.04.002). We thank Mario Senden and Joël Reithler for helpful comments on the manuscript. 


\section{References}

Alvarez, P., \& Squire, L. R. (1994). Memory consolidation and the medial temporal lobe: A simple network model. Proceedings of the National Academy of Sciences, 91(15), 7041-7045.

Been, M., Jans, B., \& De Weerd, P. (2011). Time-limited consolidation and task interference: No direct link. The Journal of Neuroscience, 31(42), 14944-14951.

Benucci, A., Saleem, A. B., \& Carandini, M. (2013). Adaptation maintains population homeostasis in primary visual cortex. Nature Neuroscience, 16(6), 724729.

Bradley, A., Skottun, B. C., Ohzawa, I., Sclar, G., \& Freeman, R. D. (1987). Visual orientation and spatial frequency discrimination: A comparison of single neurons and behavior. Journal of Neurophysiology, 57(3), 755-772.

Brashers-Krug, T., Shadmehr, R., \& Bizzi, E. (1996). Consolidation in human motor memory. Nature, 382(6588), 252-255.

Bruchmann, M., Breitmeyer, B. G., \& Pantev, C. (2010). Metacontrast masking within and between visual channels: Effects of orientation and spatial frequency contrasts. Journal of Vision, 10(6), 1-14.

Buckner, R. L., Petersen, S. E., Ojemann, J. G., Miezin, F. M., Squire, L., \& Raichle, M. (1995). Functional anatomical studies of explicit and implicit memory retrieval tasks. The Journal of Neuroscience, 15(1), 12-29.

Caithness, G., Osu, R., Bays, P., Chase, H., Klassen, J., Kawato, M., . . Flanagan, J. R. (2004). Failure to consolidate the consolidation theory of learning for sensorimotor adaptation tasks. The Journal of Neuroscience, 24(40), 8662-8671.

Dale, A. M., Liu, A. K., Fischl, B. R., Buckner, R. L., Belliveau, J. W., Lewine, J. D., \& Halgren, E. (2000). Dynamic statistical parametric mapping: Combining fmri and meg for high-resolution imaging of cortical activity. Neuron, 26(1), 55-67.

De Weerd, P., Reithler, J., van de Ven, V., Been, M., Jacobs, C., \& Sack, A. T. (2012). Posttraining transcranial magnetic stimulation of striate cortex disrupts consolidation early in visual skill learning. The Journal of Neuroscience, 32(6), 1981-1988.

Demb, J. B., Desmond, J. E., Wagner, A. D., Vaidya, C. J., Glover, G. H., \& Gabrieli, J. D. (1995). Semantic encoding and retrieval in the left inferior prefrontal cortex: A functional mri study of task difficulty and process specificity. The Journal of Neuroscience, 15(9), 5870-5878. 
Dragoi, V., Sharma, J., Miller, E. K., \& Sur, M. (2002). Dynamics of neuronal sensitivity in visual cortex and local feature discrimination. Nature Neuroscience, 5(9), 883-891.

Dragoi, V., Sharma, J., \& Sur, M. (2000). Adaptation-induced plasticity of orientation tuning in adult visual cortex. Neuron, 28(1), 287-298.

Felsen, G., Shen, Y.-s., Yao, H., Spor, G., Li, C., \& Dan, Y. (2002). Dynamic modification of cortical orientation tuning mediated by recurrent connections. Neuron, 36(5), 945-954.

Goedert, K. M., \& Willingham, D. B. (2002). Patterns of interference in sequence learning and prism adaptation inconsistent with the consolidation hypothesis. Learning and Memory, 9(5), 279-292.

Goldstone, R. L. (1998). Perceptual learning. Annual Review of Psychology, 49(1), 585-612.

Greenlee, M. W., \& Magnussen, S. (1988). Interactions among spatial frequency and orientation channels adapted concurrently. Vision Research, 28(12), 1303-1310.

Grill-Spector, K., Kushnir, T., Edelman, S., Avidan, G., Itzchak, Y., \& Malach, R. (1999). Differential processing of objects under various viewing conditions in the human lateral occipital complex. Neuron, 24(1), 187-203.

Gutnisky, D. A., \& Dragoi, V. (2008). Adaptive coding of visual information in neural populations. Nature, 452(7184), 220-224.

Gutnisky, D. A., Hansen, B. J., Iliescu, B. F., \& Dragoi, V. (2009). Attention alters visual plasticity during exposure-based learning. Current Biology, 19(7), 555-560.

Harris, H., Gliksberg, M., \& Sagi, D. (2012). Generalized perceptual learning in the absence of sensory adaptation. Current Biology, 22(19), 1813-1817.

Hebb, D. O. (1949). The organization of behavior. New York: Wiley.

Henson, R., Rylands, A., Ross, E., Vuilleumeir, P., \& Rugg, M. (2004). The effect of repetition lag on electrophysiological and haemodynamic correlates of visual object priming. Neuroimage, 21(4), 1674-1689.

Henson, R., Shallice, T., \& Dolan, R. (2000). Neuroimaging evidence for dissociable forms of repetition priming. Science, 287(5456), 1269-1272.

Jiang, Y., Haxby, J. V., Martin, A., Ungerleider, L. G., \& Parasuraman, R. (2000). Complementary neural mechanisms for tracking items in human working memory. Science, 287(5453), 643-646. 
Karni, A. (1996). The acquisition of perceptual and motor skills: A memory system in the adult human cortex. Cognitive Brain research, 5(1-2), 39-48.

Karni, A., \& Sagi, D. (1993). The time course of learning a visual skill. Nature, 365(6443), 250-252.

Kohn, A. (2007). Visual adaptation: Physiology, mechanisms, and functional benefits. Journal of Neurophysiology, 97(5), 3155-3164.

Köster, E. P., Degel, J., \& Piper, D. (2002). Proactive and retroactive interference in implicit odor memory. Chemical Senses, 27(3), 191-206.

Lewis, C. M., Baldassarre, A., Committeri, G., Romani, G. L., \& Corbetta, M. (2009). Learning sculpts the spontaneous activity of the resting human brain. Proceedings of the National Academy of Sciences, 106(41), 17558-17563.

Li, L., Miller, E. K., \& Desimone, R. (1993). The representation of stimulus familiarity in anterior inferior temporal cortex. Journal of Neurophysiology, 69, 1918-1918.

Luft, A. R., Buitrago, M. M., Ringer, T., Dichgans, J., \& Schulz, J. B. (2004). Motor skill learning depends on protein synthesis in motor cortex after training. The Journal of Neuroscience, 24(29), 6515-6520.

Macknik, S. L., \& Livingstone, M. S. (1998). Neuronal correlates of visibility and invisibility in the primate visual system. Nature Neuroscience, 1(2), 144-149.

McGaugh, J. L. (1966). Time-dependent processes in memory storage. Science, 153(3742), 1351-1358.

Miller, E. K., \& Desimone, R. (1994). Parallel neuronal mechanisms for short-term memory. Science, 263(5146), 520-522.

Müller, G. E., \& Pilzecker, A. (1900). Experimentelle Beiträge zur Lehre vom Gedächtnis. Zeitschrift für Psychologie und Physiologie der Sinnesorgane, (Suppl. No. 1).

Müller, J. R., Metha, A. B., Krauskopf, J., \& Lennie, P. (1999). Rapid adaptation in visual cortex to the structure of images. Science, 285(5432), 1405-1408.

Naccache, L., \& Dehaene, S. (2001). The priming method: Imaging unconscious repetition priming reveals an abstract representation of number in the parietal lobes. Cerebral Cortex, 11(10), 966-974.

Nadel, L., \& Land, C. (2000). Commentary - reconsolidation : Memory traces revisited. Nature reviews Neuroscience, 1(3), 209-212. 
Nader, K., Schafe, G. E., \& Le Doux, J. E. (2000). Fear memories require protein synthesis in the amygdala for reconsolidation after retrieval. Nature, 406(6797), 722-726.

Nishina, S., Seitz, A. R., Kawato, M., \& Watanabe, T. (2007). Effect of spatial distance to the task stimulus on task-irrelevant perceptual learning of static gabors. Journal of Vision, 7(13), 1-10.

Polat, U., \& Sagi, D. (1994). Spatial interactions in human vision: From near to far via experience-dependent cascades of connections. Proceedings of the National Academy of Sciences, 91(4), 1206-1209.

Sathian, K., \& Zangaladze, A. (1997). Tactile learning is task specific but transfers between fingers. Attention, Perception, \& Psychophysics, 59(1), 119-128.

Schoups, A. A., Vogels, R., \& Orban, G. A. (1995). Human perceptual learning in identifying the oblique orientation: Retinotopy, orientation specificity and monocularity. Journal of Physiology, 483(Pt 3), 797-810.

Schoups, A. A., Vogels, R., Qian, N., \& Orban, G. A. (2001). Practising orientation identification improves orientation coding in v1 neurons. Nature, 412(6846), 549553.

Seitz, A. R. (2005). Task-specific disruption of perceptual learning. Proceedings of the National Academy of Sciences, 102(41), 14895-14900.

Seitz, A. R., \& Watanabe, T. (2005). A unified model for perceptual learning. Trends in Cognitive Sciences, 9(7), 329-334.

Seitz, A. R., \& Watanabe, T. (2009). The phenomenon of task-irrelevant perceptual learning. Vision Research, 49(21), 2604-2610.

Seitz, A. R., Yamagishi, N., Werner, B., Goda, N., Kawato, M., \& Watanabe, T. (2005). Task-specific disruption of perceptual learning. Proceedings of the National Academy of Sciences, 102(41), 14895-14900.

Shadmehr, R., \& Holcomb, H. H. (1997). Neural correlates of motor memory consolidation. Science, 277(5327), 821-825.

Shadmehr, R., \& Mussa-Ivaldi, F. (1994). Adaptive representation of dynamics during learning of a motor task. The Journal of Neuroscience, 14(5), 3208-3224.

Sobotka, S., \& Ringo, J. L. (1996). Mnemonic responses of single units recorded from monkey inferotemporal cortex, accessed via transcommissural versus direct pathways: A dissociation between unit activity and behavior. The Journal of Neuroscience, 16(13), 4222-4230. 
Stern, C. E., Corkin, S., Gonzalez, R. G., Guimaraes, A. R., Baker, J. R., Jennings, P. J., . . . Rosen, B. R. (1996). The hippocampal formation participates in novel picture encoding: Evidence from functional magnetic resonance imaging. Proceedings of the National Academy of Sciences, 93(16), 8660-8665.

Tambini, A., Ketz, N., \& Davachi, L. (2010). Enhanced brain correlations during rest are related to memory for recent experiences. Neuron, 65(2), 280-290.

Teich, A. F., \& Qian, N. (2003). Learning and adaptation in a recurrent model of v1 orientation selectivity. Journal of Neurophysiology, 89(4), 2086-2100.

Teich, A. F., \& Qian, N. (2010). V1 orientation plasticity is explained by broadly tuned feedforward inputs and intracortical sharpening. Visual Neuroscience, 27(1-2), 57-73.

Tsushima, Y., Seitz, A. R., \& Watanabe, T. (2008). Task-irrelevant learning occurs only when the irrelevant feature is weak. Current Biology, 18(12), R516-R517.

van Turennout, M., Ellmore, T., \& Martin, A. (2000). Long-lasting cortical plasticity in the object naming system. Nature Neuroscience, 3(12), 1329-1334.

Vogels, R. (1990). Population coding of stimulus orientation by striate cortical cells. Biological Cybernetics, 64(1), 25-31.

Watanabe, T., Náñez, J. E., \& Sasaki, Y. (2001). Perceptual learning without perception. Nature, 413(6858), 844-848.

Wetherill, G. B., \& Levitt, H. (1965). Sequential estimation of points on a psychometric function. British Journal of Mathematical and Statistical Psychology, 18(1), 1-10.

Zhang, J.-Y., Kuai, S.-G., Xiao, L.-Q., Klein, S. A., Levi, D. M., \& Yu, C. (2008). Stimulus coding rules for perceptual learning. PLoS Biol, 6(8), e197. 


\section{ChAPTER 6}

The attentional blink effect is related to microsaccade suppression but not rebound

Roberts, M. J.*, Lange, G.*, Lowet, E., \& De Weerd, P. (in preparation). The attentional blink effect is related to microsaccade suppression but not rebound.

*shared first authorship 


\begin{abstract}
The visibility of stimuli is influenced by eye movements and by attention, and, moreover, the rate and direction of eye movements are strongly influenced by attentional deployment. We used the classic attentional blink paradigm to investigate whether reduced target detectability following discrimination of a preceding target was related to changes in the rate of microsaccades. Following the detection of a target, attentional resources are thought to be briefly depleted, suppressing the detectability of additional targets. We found a robust suppression of microsaccade occurrence during the period associated with reduced performance in the detection of the second target, 200 to $300 \mathrm{~ms}$ after the presentation of the first target. Following the period of MS suppression we found a period of rate enhancement lasting 200ms. The extent of MS suppression and rebound was significantly correlated with participants' performance at detecting the second target at the second lag interval, when the suppression in the detection of the second target was greatest. These results indicate that the attentional blink is related to a reduction in microsaccade rate. The data suggest that the reduced microsaccade rate may represent an index of the temporary withdrawal of attentional resources thought to underlie the attentional blink, but also open up alternative explanations unrelated to attention.
\end{abstract}




\section{Introduction}

Microsaccades (MSs) are small involuntary eye movements which take place during attempted fixation. Their occurrence is crucial for maintaining perception as a perfectly stable image on the retina quickly fades (e.g., Riggs, Ratliff, Cornsweet, \& Cornsweet, 1953) (for reviews see Martinez-Conde, Macknik, \& Hubel, 2004; Rolfs, 2009). Recent research has shown that perception of peripheral and foveal targets may fade after a period of 200 to $600 \mathrm{~ms}$ without a microsaccade (Costela, McCamy, Macknik, Otero-Millan, \& Martinez-Conde, 2013; Hsieh \& Tse, 2009b; Martinez-Conde, Macknik, Troncoso, \& Dyar, 2006; McCamy, Macknik, \& Martinez-Conde, 2014; Rucci \& Desbordes, 2003; Troncoso, Macknik, \& MartinezConde, 2008) (but see Bonneh et al., 2010). However perception is also compromised around the moment of the movement (Hafed, 2013; Hass \& Horwitz, 2011; Maij, Matziridi, Smeets, \& Brenner, 2012; Schütz, Braun, \& Gegenfurtner, 2009) and it may therefore be adaptive to briefly suppress microsaccades during demanding visual tasks (Bridgeman \& Palca, 1980). While microsaccade typically occur at a relatively stable rate of 1-4Hz (Bosman, Womelsdorf, Desimone, \& Fries, 2009; Engbert \& Kliegl, 2003; Gonzalez, Wong, Niechwiej-Szwedo, Tarita-Nistor, \& Steinbach, 2012; Otero-Millan, Troncoso, Macknik, Serrano-Pedraza, \& Martinez-Conde, 2008a), visual input (J. Cui, M. Wilke, N. K. Logothetis, D. A. Leopold, \& H. Liang, 2009; Gowen, Abadi, \& Poliakoff, 2005; Hsieh \& Tse, 2009a) and cognitive factors (Betta \& Turatto, 2006; Gowen et al., 2005; Kohama, Endoh, $\&$ Yoshida, 2013) are also known to influence their occurrence.

The onset of a visual stimulus has been widely reported to lead to a rapid reduction of microsaccades lasting between 100 and 500ms followed by a rebound and overshoot occurring 200 to $600 \mathrm{~ms}$ after stimulus onset (Betta \& Turatto, 2006; Hafed \& Ignashchenkova, 2013; Rolfs, 2009; Rolfs, Engbert, \& Kliegl, 2005; Rolfs, Kliegl, \& Engbert, 2008a). The same 'microsaccade rate signature' has been observed following the onset of a visual oddball in a stream (Valsecchi, Betta, \& Turatto, 2007; Valsecchi \& Turatto, 2009), especially when the oddball was behaviorally relevant (Valsecchi, Dimigen, Kliegl, Sommer, \& Turatto, 2009), and by the onset of endogenous or exogenous attentional cues (Gowen, Abadi, Poliakoff, 
Hansen, \& Miall, 2007; Laubrock, Engbert, \& Kliegl, 2005; Rolfs et al., 2005). While the initial suppression is quite stereotypical (Rolfs, Kliegl, \& Engbert, 2008b), the later rebound is sensitive to cognitive (Engbert, 2006; Gowen et al., 2007; Laubrock et al., 2005) and stimulus (Engbert \& Kliegl, 2003) variables, and may be absent in certain conditions (J. Cui et al., 2009; Valsecchi et al., 2007; Valsecchi \& Turatto, 2009). Sustained attention has been associated with a reduction of microsaccade rate which can last several seconds (Kohama et al., 2013; Pastukhov \& Braun, 2010) (for review see Rolfs, 2009). Interestingly, the few microsaccades which do occur during periods of sustained attention have a direction bias towards (Betta \& Turatto, 2006; Engbert \& Kliegl, 2003; Hafed \& Clark, 2002; Kohama \& Usui, 2002; Laubrock et al., 2005; Laubrock, Kliegl, Rolfs, \& Engbert, 2010; Pastukhov \& Braun, 2010; Yuval-Greenberg, Merriam, \& Heeger, 2014) or away from (Galfano, Betta, \& Turatto, 2004; Gowen et al., 2007; Laubrock et al., 2005; Rolfs, Engbert, \& Klieg1, 2004; Rolfs et al., 2005; Tse, Sheinberg, \& Logothetis, 2003) the attended object, prompting some authors to suggest that microsaccades may be used as an objective index of attention (Engbert \& Kliegl, 2003; Hafed \& Clark, 2002; Laubrock, Engbert, Rolfs, \& Kliegl, 2007; YuvalGreenberg et al., 2014) (but see Horowitz, Fine, Fencsik, Yurgenson, \& Wolfe, 2007; Tse, 2004; Tse, Sheinberg, \& Logothetis, 2002) whereby a low rate and consistent direction corresponds to states of attention.

Attention is understood as a mechanism for highlighting behaviorally relevant stimuli for preferential processing, a process involving the enhanced processing of target stimuli and suppressed processing of distractors (Desimone \& Duncan, 1995). Attention is thus a mechanism to overcome the brain's limited processing capacity. One striking demonstration of this limited capacity is seen in the attentional blink $(\mathrm{AB})$ paradigm. Here, participants are required to respond to two items embedded in a rapid serial visual presentation (RSVP) sequence. If the second stimulus is presented within the specific time window of 150 to $250 \mathrm{~ms}$ after the first, detection and discrimination of the second stimulus is impaired. While a host of theories have been presented to account for this effect (for a review see Dux \& Marois, 2009) a consistent theme has been that correctly identifying the targets requires defined processing resources, including encoding, working memory, response selection and 
above all attention, which are required to enhance the target representation and suppress distractor representations. In the $\mathrm{AB}$ paradigm, processing of the first target (T1) is thought to render these resources temporarily unavailable, thereby impairing the identification of the second target (T2) for a brief period.

Since microsaccades have a role in both attention and perceptual performance we questioned what role they would play in the $\mathrm{AB}$ effect. We reasoned that as the participants anticipated the presentation of T1, microsaccades would be suppressed, but that during the attentional blink window the temporary withdrawal of attention would allow microsaccade rate to rebound to levels observed during inattentive states. We reasoned that during the hypothesized period of high microsaccade rate there would be a high probability of $\mathrm{T} 2$ presentations falling at the moment of a MS, and consequently being unperceived.

We found that microsaccade rate was indeed modulated in the $\mathrm{AB}$ paradigm, however contrary to our hypotheses we found that the period of poor perceptual performance was associated with a marked reduction of microsaccade rate. Moreover, the amount of microsaccade suppression was positively linked to the magnitude of the $\mathrm{AB}$ effect, whereby participants who showed the greatest behavioral deficit also showed the largest reduction in MS rate. Following the microsaccade rate signature we also found a robust rebound in microsaccade rate after the period of suppression. In the rebound period microsaccade rate approximately doubled compared to the pre-T1 period, however T2 performance was not impaired during this period. Our results imply a contradiction between the view that low MS rate corresponds to states of heightened attention and the view that impaired performance at a specific period of the $\mathrm{AB}$ paradigm corresponds to temporarily unavailable attentional resources. 


\section{Methods}

\section{Participants}

22 participants (16 female; mean age 22.77, SD 2.52), naïve to the purpose of the experiment were recruited for this study. All participants had normal or corrected-tonormal visual acuity. After full information about all procedures, informed, written and verbal consent was obtained according to the Helsinki Declaration. Participants were also informed about their right to withdraw participation at any time. All procedures were approved by the local Ethical Committee of the Faculty of Psychology and Neuroscience (ECP). For their participation in the study participants received either monetary reward or credits to fulfill course requirements.

\section{Stimuli, Apparatus, and Task}

Data collection took place in a dimly lit room while participants were sitting in a chair with their head supported by a chin and head rest to keep eye-screen distance constant at $57 \mathrm{~cm}$. Visual stimuli were displayed on a 19" Samsung SyncMaster 940BF LCD monitor (Samsung, Seoul, South Korea; 60Hz refresh rate, 1280x1024 resolution). Eye movements were recorded monocularly with a desktop-mounted Eyelink 1000 eyetracker (SR Research Ltd., Mississauga, Ontario, Canada; 500Hz or $1000 \mathrm{~Hz}$ sampling rate, $<0.01^{\circ} \mathrm{RMS}$ spatial resolution). Stimulus presentation and response recording was performed by Cortex v.5.9.6 (NIH freeware for psychophysical and neurophysiological experimentation). Exact stimulus timing was derived from a photodiode attached to the left border of the monitor.

Participants were shown a RSVP stream of dark grey capital letters (luminance: $11.73 \mathrm{~cd} / \mathrm{m}^{2}$ ) on a light gray background (luminance: $39.59 \mathrm{~cd} / \mathrm{m}^{2}$ ) in the center of the screen. Their task was to identify the only red letter in the stream which could be either a ' $\mathrm{T}$ ' or an ' $\mathrm{L}$ ' (referred to as $\mathrm{T} 1$ ), and to indicate whether a black ' $\mathrm{X}$ ' (referred to as T2) was presented anywhere after T1. There were nine different experimental conditions: T2 could either be presented 130, 260, 390, 520, 650, 780, or $910 \mathrm{~ms}$ after T1 (referred to as lags 1-7), only T2 could be omitted, or both T1 and $\mathrm{T} 2$ could be omitted. Each condition occurred with the same likelihood, so that there were $22 \%$ of all trials without T2. The RSVP letter stream consisted of 22 letters (Figure 1A), which were presented in the center of the screen and were $0.3^{\circ}$ large. 
The grey distractor letters were randomly chosen from the alphabet excluding the letters T, L, X, and Q; immediate repetition of a letter was not possible. T1 was presented at randomly between position 10 and 14 inclusive, and consequently, T2 could appear anywhere between positions 11 and 21 depending on the time lag between $\mathrm{T} 1$ and $\mathrm{T} 2$. After each trial the response options for $\mathrm{T} 1$ (' $\mathrm{T}$ ', ' $\mathrm{L}$ ' and 'no letter') and T2 ('no X' and ' $\mathrm{X}$ ') were displayed on the screen and participants could indicate their response with the left (indicating either ' $L$ ' as $T 1$ or 'no $X$ ' for $T 2$ ), right (indicating either ' $\mathrm{T}$ ' as $\mathrm{T} 1$ or ' $\mathrm{X}$ ' as $\mathrm{T} 2$ ), and down (neither ' $\mathrm{T}$ ' nor ' $\mathrm{L}$ ' was seen as T1) arrows on a standard computer keyboard that corresponded spatially to the display of possible answers (see Figure 1B). For the left, down and right arrow

A

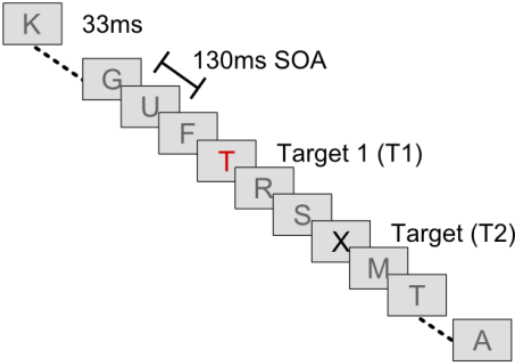

B

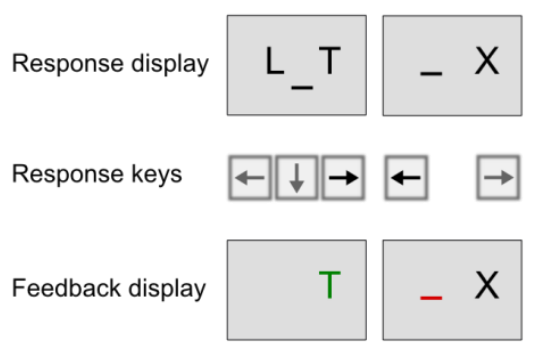

Figure 1. An exemplary trial and response screen. $\boldsymbol{A}$, One trial consisted of a stream of 22 capital letters. Grey letters were distractors, the red letter target 1 (T1), and the black X was target 2 (T2). Each letter was shown for 33ms, with a stimulus onset asynchrony (SOA) of $130 \mathrm{~ms}$. Note: Letters are enlarged for illustratory purposes and were only $0.3^{\circ}$ large in the experimental display. $\boldsymbol{B}$, Response display, response keys and exemplary feedback display for T1 (left) and T2 (right) are shown. The black arrow on the displayed keys signifies an imaginary response given for this trial and the resulting feedback that is given is shown below. 
key participants used their index, middle, and ring finger of their right hand respectively. Feedback was provided by highlighting the correctly given answer in green and removing incorrect options or, in case of an incorrect answer, highlighting the given response in red and showing the correct answer in black. During the experiment, the color of the two target letters $\mathrm{T} 1$ and $\mathrm{T} 2$ was adjusted with a Wetherill \& Levitt staircase procedure tracking $84 \%$ correct performance. So, with more proficiency on the task, the color of T1 and T2 would become slightly greyer, and more similar to the non-target letters in the RSVP stream, by reducing the value of the red channel only for T1, or reducing all three channels for T2 (in steps of 10). By doing so, the difficulty level of the T1 and T2 tasks was adaptively set at a challenging but doable level. The staircase ran independently for the T1 and T2 targets, but adjustments were made after correct responses or mistakes irrespective of the lag conditions selected randomly trial-by-trial, such that performance was overall kept at an level of $\sim 84 \%$ across all lags.

\section{Procedure}

The experiment consisted of four blocks with 90 trials each (i.e., 10 trials per condition per run and 40 trials per condition in total), resulting in a total of 360 trials. Blocks were separated by a short break. A trial started by $300 \mathrm{~ms}$ of fixation during which participants were required to keep their gaze within a $3^{\circ}$ by $3^{\circ}$ window centered on the fixation cross, which would then start the RSVP stream. If the gaze shifted beyond that window anytime during the trial, the trial was immediately aborted and a new trial would start. Aborted trials were repeated at a later time in the block. Each letter was presented for $33 \mathrm{~ms}$ with a stimulus onset asynchrony of $130 \mathrm{~ms}$, resulting in a total duration of each trial of 3886ms. After the RSVP stream, a response screen for T1 was presented. After participants had indicated their response at their own pace, a feedback screen was shown. Subsequently, the response screen for $\mathrm{T} 2$ was shown, which after the response by the participant again was followed by a second feedback screen.

Before starting the experiment, participants completed 18 training trials (i.e., two per condition) to familiarize with the task. Afterwards their dominant eye was determined from which eye movements were recorded during the experiment. Eye position was calibrated using a nine-point fixation procedure. The calibration was 
regarded successful if the validation procedure resulted in an average gaze-position error of less than $0.5^{\circ}$ and a maximum error of less than $1^{\circ}$. If necessary, calibration was repeated between blocks.

\section{Data and Analyses}

Microsaccades were identified using the algorithm put forward by Engbert and Kliegl (2003) in MATLAB (MathWorks, Natick, Massachusetts, U.S.A). Here the time series of eye positions was transformed into velocities calculated over a moving window of seven samples. A microsaccade was defined as the period in which the following conditions were satisfied: (1) the angular eye velocity exceeded a combined threshold, which was four times the median-based standard deviation of the velocity distribution $(\lambda=4)$, computed independently for horizontal and vertical components and separately for each trial; (2) the microsaccade duration exceeded a minimum of $7 \mathrm{~ms}$. Microsaccade times were defined by the start time of the eye movement.

To verify the validity of the detected microsaccades, we examined whether the eye movements classified as microsaccades satisfied the main-sequence criterion (Zuber, Stark, \& Cook, 1965), which requires a linear correlation between peak saccadic velocity and amplitude and verifies the ballistic nature of saccade
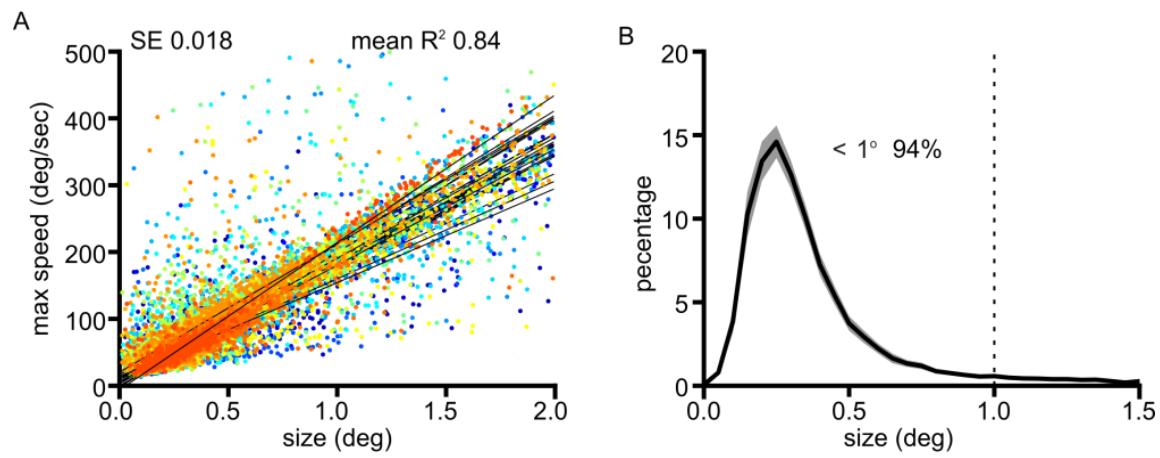

Figure 2. Quantification of MS statistics. A, Microsaccade main sequence; MS size was linearly related to peak velocity. Each dot corresponds to an individual MS, colors correspond to different subjects. Correlations were high in all individual participants as well (see text). $\boldsymbol{B}$, Histogram of average MS size, line width shows standard error. Over all subjects, $94 \%$ of MS were of less than 1 degree. 
execution. The results showed consistent linear relationship supported by high correlation coefficients, consistent with previous findings (Betta, Galfano, \& Turatto, 2007; Engbert \& Kliegl, 2003; Galfano et al., 2004; Møller, Laursen, Tygesen, \& Sjølie, 2002). Therefore, the linear relationship verified that the events detected by the algorithm were valid microsaccades (Figure 2).

T1 onset time was randomized over trials (see Stimuli, Apparatus, and Task and Figure 3). To account for the variation in microsaccade rate observed at the 5 possible T1 onset times, we subtracted the trial-start locked microsaccade rate from the rate observed around $\mathrm{T} 1$ on each given trial, leaving only the variation in microsaccade rate unique to $\mathrm{T} 1$ presentation and not related to general changes in rate over the trial. To facilitate this operation microsaccade times were convolved with a Gaussian kernel with a full width at half maximum of $30 \mathrm{~ms}$ to produce an estimate of instantaneous microsaccade rate per individual trial, as is often used in spike-train analysis (Lehky, 2009). Thus, for each trial we subtracted the average trial-start locked rate, calculated for all trials except the current trial of interest, from the T1 locked MS rate on that trial. MS rates are presented as MS rate corrected by the stimulus onset locked average, unless otherwise stated. We observed a reduction in MS rate towards the middle of the trial and recovery towards the end.

This pattern could be well fit with an asymmetric Gaussian curve with the form:

$$
Y=\text { gain } \times \exp \left(2 \times\left[\frac{X(1: c t r)-\text { peak }}{\text { sigma } 1} \frac{X(c t r+1: \text { end })-\text { peak }}{\text { sigma } 2}\right]^{2}\right)+\text { offset }
$$

Where gain is a scaling factor reflecting the height of the Gaussian, peak is the center of the Gaussian in ms, ctr is the index in the array of the center, sigmal and sigma2 are the widths of the left and right sides of the Gaussian and offset is the offset from 0 . To fit the curve to the data we adjusted the free parameters to minimize the summed squared error using the Matlab Fminsearch function. 


\section{Task performance}

Behavioral performance in the T2 identification task was calculated as proportion correct among trials where the $\mathrm{T} 1$ was correctly identified. To facilitated comparison over participants we normalized performance for T2 lags 1 to 6 by performance at lag 7.

\section{Statistical testing}

We were interested to test how presentation of the T1 influenced MS rate on a moment to moment basis and whether this temporal variation in T1-locked MS rate correlated with T2 detection rate. These tests were made using sliding window analysis, correction for multiple comparisons (the number of window positions) was achieved using a permutation methods as implemented in the Fieldtrip Matlab toolbox (Litvak et al., 2011) and described in detail by Maris \& Oostenveld (2007). We first tested for significant differences between subjects' mean MS rate profile following T1 onset and their mean MS rate profile following randomly selected distractors. For each time point we calculated the significance of the paired t-test Tvalue using the Monte-Carlo permutation distribution after 1000 random resamplings and Bonferroni correction for multiple comparisons. We next tested the relationship between subjects' MS rate around T1 presentation and their behavioral performance. We calculated the correlation between MS rate and performance at the T1 and T2 tasks. We calculated the significance of the correlation using the MonteCarlo permutation distribution after 1000 random resamplings and Bonferroni correction for multiple comparisons. For both analyses we used a $133 \mathrm{~ms}$ window length to calculate mean MS rate, we choose this window length to match the interstimulus interval in the task. 


\section{Results}

\section{Task performance}

Figure 3 shows the suppression of the detection of the second target at different time intervals following the identification of the first target in the $\mathrm{AB}$ paradigm as implemented in our study (raw performance in Figure 3A, and normalized performance in 3B). We saw in the average data the suppression of $\mathrm{T} 2$ detection as expected from the literature (black solid lines in Figure panels), but we also found large variability in individual participants (as indicated in the color-coded T2 performance levels per participant). In following analysis, we set out to relate this variability in $\mathrm{T} 2$ detection performance to variations in microsaccades rate variations.

A
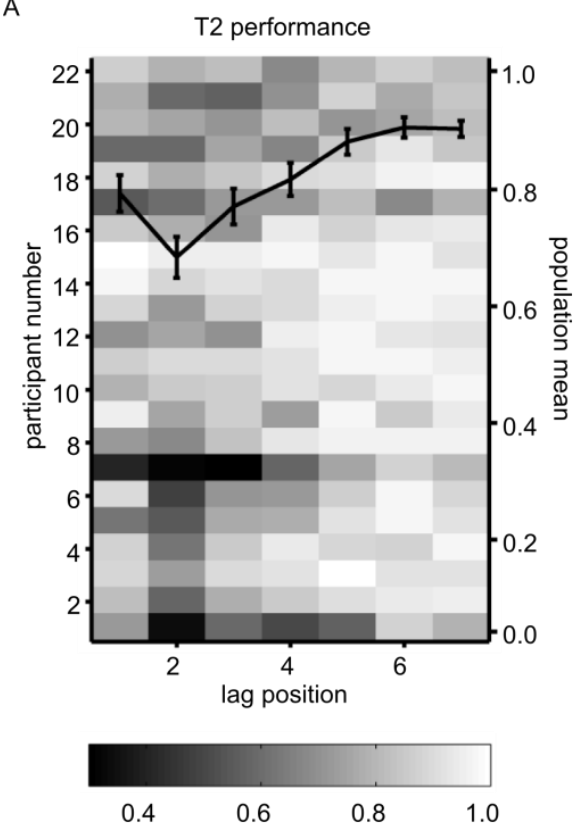

B

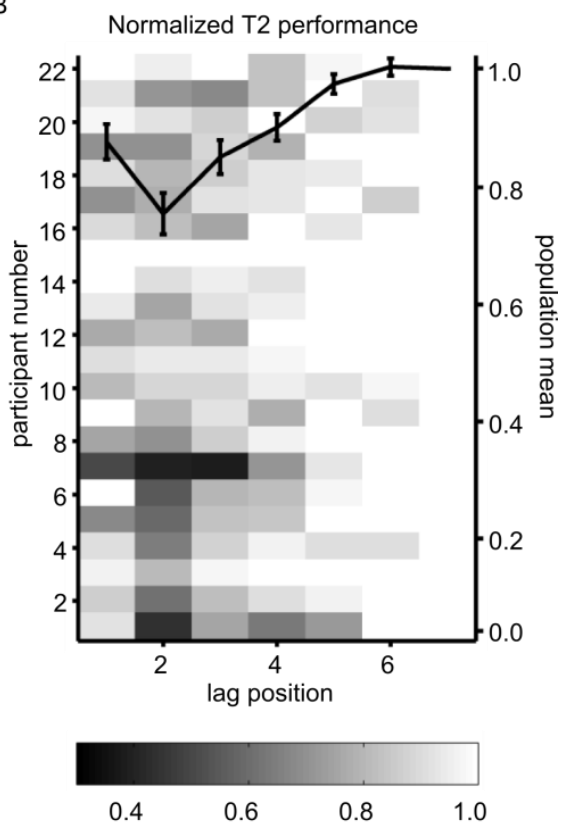

Figure 3. $T 2$ task performance raw $(\boldsymbol{A})$ and normalized by performance at lag $7(\boldsymbol{B})$. Colored background corresponds to individual participant performance (participant number on left y axis). Overlaid black line corresponds to population average (errorbars \pm SEM) 


\section{Microsaccade rate modulation over trial}

We first examined MS rate over the course of the trial (Figure 4A). We found a pronounced reduction of MS rate in the middle time points compared to the start of the trial and a slight recovery towards the end. We found in the total population of participants $(\mathrm{N}=22)$ that $\mathrm{MS}$ rate (per second) was $2.2(\mathrm{SD}=0.9)$ during the $300 \mathrm{~ms}$ pre-stimulus period, $1.3(\mathrm{SD}=0.5)$ during the first $500 \mathrm{~ms}, 0.53(\mathrm{SD}=0.3)$ during the middle of the trial $(1500-2000 \mathrm{~ms})$, and $0.6(\mathrm{SD}=0.3)$ during the last500ms. The suppression of MS rate in the middle part of the trial (coinciding with the time in which T1 and T2 were presented) is in line with Pastukhov and Braun (2010) who have shown a MS reduction during sustained attention. In addition, we found changes in MS size and speed over the course of the trial (Figure 4 B, 4C). We also observed a sharp suppression and rebound of MS rate close to the start of the trial, which is a signature modulation of MS at the beginning of trials that has been reported also by others (Betta \& Turatto, 2006; Hafed \& Ignashchenkova, 2013; Rolfs, 2009; Rolfs et al., 2005; Rolfs et al., 2008a). Since this MS rate signature occurred against a background of rapidly reducing MS rate we quantified the MS rate signature by fitting an asymmetric Gaussian (methods) first to the populationmean data (red curve, Figure 4A) after which we determined points along the curve with the highest residuals. The asymmetric Gaussian gave a good fit to the data, explaining $81 \%$ percent of the variance. The trial-onset related microsaccade rate signature as expected could be readily identified as time points with high residuals from the fitted curve. For a quantitative threshold we z-scored the residuals and identified time points with absolute z-scores of more than 2 (highlighted regions in Figure 3). One region defined by negative residuals and corresponding to MS rate suppression was centered at $148 \mathrm{~ms}$ after stimulus onset. A second region characterized by positive residuals and corresponding to MS rate rebound was centered at $334 \mathrm{~ms}$ after stimulus onset. There was considerable variation in the MS rate signature among participants. An analysis on the individual participant data $(\mathrm{N}=22)$ revealed that 18 participants exhibited MS suppression and 15 participants exhibited MS rebound, 14 subjects showed both suppression and rebound. Note that also the speed, size and direction of microsaccades changed in the course of the trial (Figure 4 B, 4C, and 4D). 
Figure 4A shows the possible T1 onset times as vertical dashed line. T1 onsets were randomized among these 5 positions in the stream (positions 10 to 14). These positions were well away from the fast dynamics associated with the start of the

A
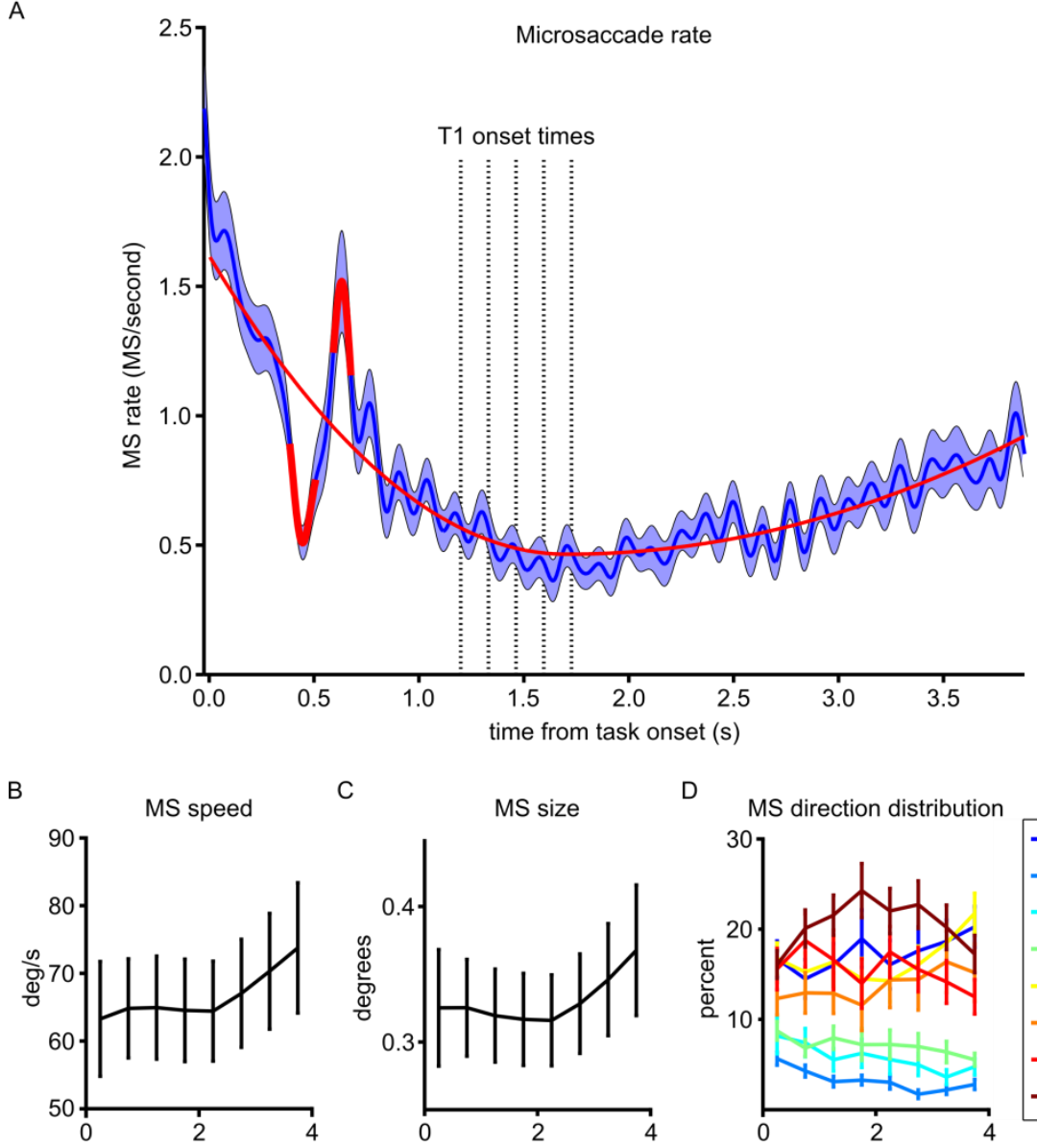

C

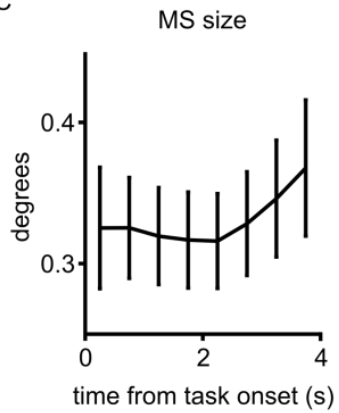

D MS direction distribution

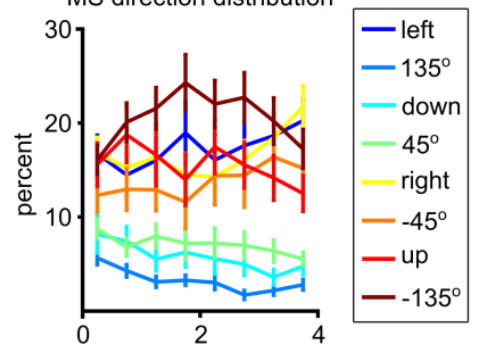

Figure 4. Microsaccade statistics as a function of duration in the trial across the population of participants $(\boldsymbol{A})$, with trials aligned to first stimulus onset. Blue line shows mean, line thickness shows standard error. T1 presentation time was randomized to 5 places in the RSVP stream, indicated by vertical lines. Thin red lines shows fitted function, thick red highlighting shows time periods with significant deviation from the fit (residuals Z-score >2). (B) Shift in mean MS speed as a function trial time. $(\boldsymbol{C})$ Shift in MS size over the trial (error bars \pm SEM). (D) MS direction distribution as a function of trial time. 
trial; however MS rate was also not stationary during this period (comparison of MS rate at position 10 and position 14 , paired t-test, $\left.t_{(21)}=6.5, p<0.001\right)$. In order to test for MS rate changes associated with the T1 onset we subtracted the stimulus onset locked mean MS rate observed from $500 \mathrm{~ms}$ before to 1 second after the time of $\mathrm{T} 1$ onset from the observed rate on a trial by trial basis. This is referred to as corrected MS rate.

\section{Microsaccade rate relative to $\mathrm{T1}$}

After aligning the MS rate to T1 onset and correcting the observed rate by the mean, we observed that the average MS rate followed the MS rate signature (Figure 5, blue line and shading area), which in trial-onset aligned trials is visible in early parts of the trial (Figure 4). Maximum suppression of MS rate was observed at $316 \mathrm{~ms}$ after $\mathrm{T} 1$ while the maximum rebound was observed at $464 \mathrm{~ms}$ after $\mathrm{T} 1$. We did not find consistent changes in MS size, speed or direction during the period around T1 onset (Figure 5B and 5C). We had initially hypothesized that the timing of the rebound phase would align with the maximum reduction in T2 performance (observed at lag 2, 266ms after T1, solid black line and error bars Figure 5). However, to the contrary, the phase with suppressed microsaccade rate coincided with the reduction in T2 performance. Equally surprisingly, the rebound phase of the MS rate signature fell in the period of rising T2 performance, whereas we had anticipated that high MS rate would be detrimental for the task of detecting very briefly presented elements.

To quantify the observed changes in MS rate we compared MS rate aligned to the T1 onset with MS rate aligned to the onset of a distractor (Figure 5, green line and shaded area). Distractor onsets at which data were aligned were chosen at random on each trial from positions 10 to 14 . We compared the T1 aligned data with the distractor aligned data using a sliding window T-test with a permutation test for correction of multiple comparisons (Maris \& Oostenveld, 2007). Similar results were also obtained by comparing the $\mathrm{T} 1$ aligned data for deviation from zero. Regions with significant deviation from the distractor aligned data are highlighted in Figure 5. We found three regions, the first corresponding to a reduction in MS rate from 160 to $344 \mathrm{~ms}$ after $\mathrm{T} 1$ onset, the second corresponding to an increase (rebound) from 400 to 580, and a third corresponding to another decrease from 650 to $800 \mathrm{~ms}$ after $\mathrm{T} 1$ onset. 
A
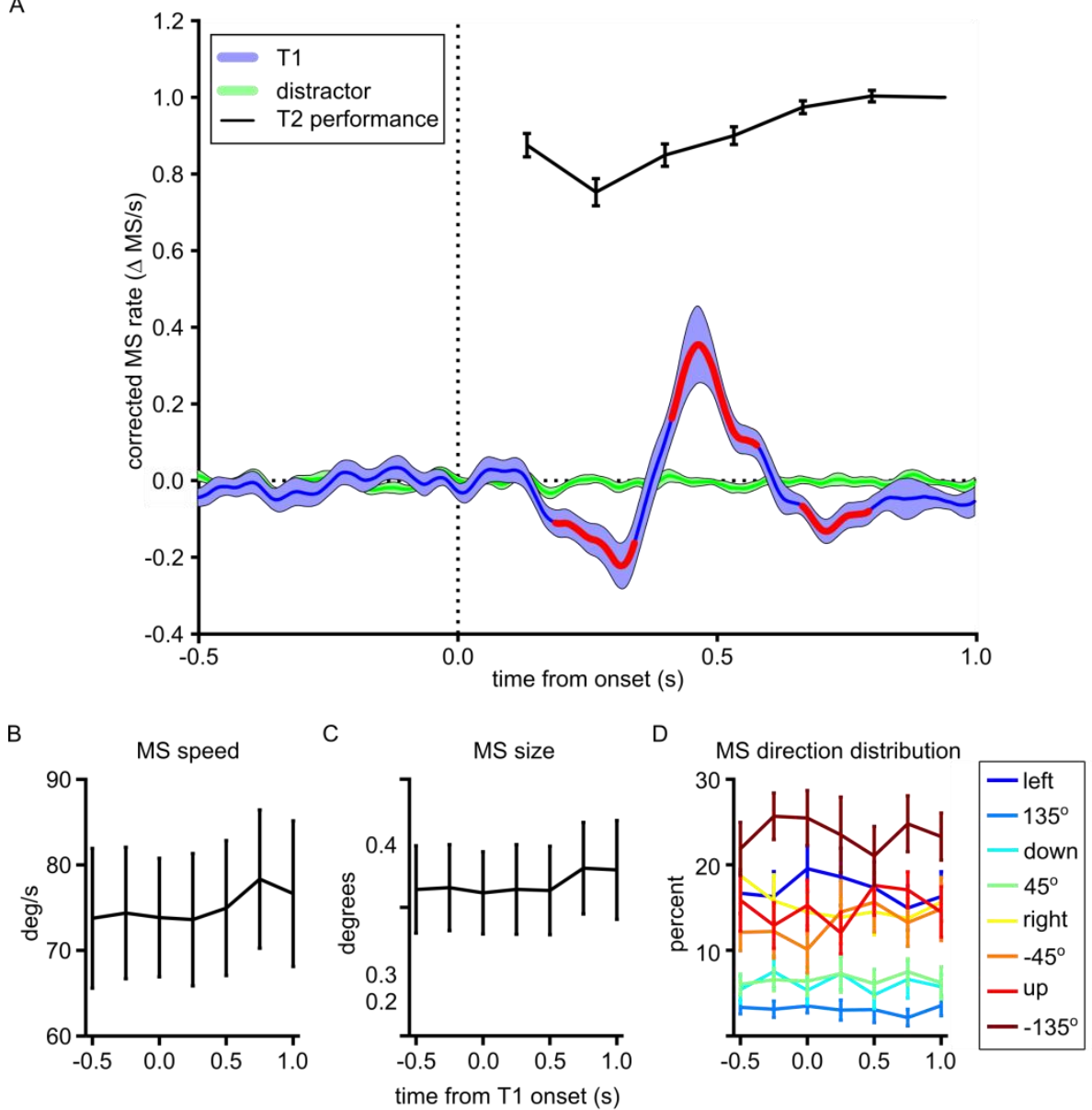

Figure 5. $\boldsymbol{A}$, Variation in MS rate after T1 (blue) and distractor onset in the total population of trials in all participants aligned to $\mathrm{T} 1$ onset. Line width shows standard error. Red highlighting shows time periods of significant difference. Black line and error bars shows T2 performance (mean, error bars standard error) normalized by performance at lag 7. (B) Shift in mean MS speed as a function T1 onset time. $(\boldsymbol{C})$ Shift in MS size as a function of T1 onset time (error bars \pm SEM). (D) MS direction distribution as a function of T1 onset time.

\section{Median split of $A B$ participants}

We sought to relate the level of MS rate with task performance. To this end we first divided the participants on the basis of a median split of the MS rate observed at different time periods relative to $\mathrm{T} 1$ presentation (shaded areas, Figure 6). Data from participants with a relatively high MS rate in the time of interest are represented in 
red, while data from participants with a relatively low rate are shown in blue. In most cases (i.e. time periods) the behavioral performance of the two groups was very similar (overlapping red and blue error bars in behavioral data). However when sorting participants on the basis of MS rate observed during the period around the
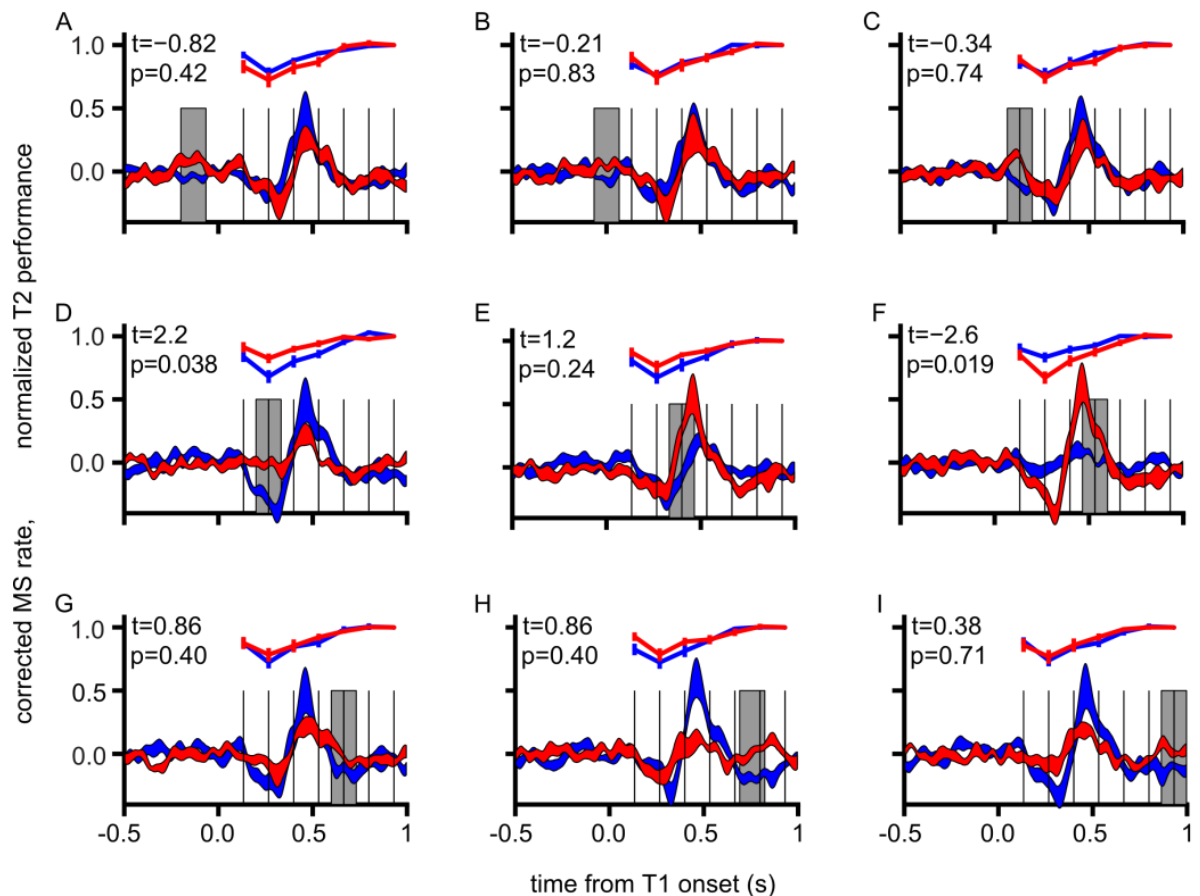

Figure 6. Sorting subjects by MS behavior reveals the importance of the suppressive phase of the MS rate signature. Subjects were divided on the basis of a median split of the relative MS rate observed in a $133 \mathrm{~ms}$ time window (grey shading). The location of the window was shifted in steps of $133 \mathrm{~ms}$ from $133 \mathrm{~ms}$ before T1 onset until $931 \mathrm{~ms}$ after T1 onset (subplots, subplot titles). Data from subjects with a high relative MS rate are shown in red, data from subjects with a low rate in blue. Performance in the T1 task is shown by a dot \pm standard error, performance in $\mathrm{T} 2$ task (normalized by performance at lag 7 ) is shown with lines \pm standard error. Comparison of performance at lag 2 with performance at all other lags with a 2 sample t-test ( $p$ lag2 $=$ rest, in red or blue text) showed significant differences in all cases. T2 performance at lag 2 in each group was compared ( $p$ difference, in black text) showed not significance difference except in the case of sorting subjects on the basis of relative MS rate around the lag $2 \mathrm{~T} 2$ presentation. 
lag 2 T2 presentation time there was a striking separation (Figure 6D, E). Participants with higher MS rate (i.e. lack of MS suppression) showed higher performance in the T2 task. This difference was significant for lag $2 \mathrm{~T} 2$ performance (high MS rate group: mean T2 performance $=0.82, S D=0.13$; low MS rate group: mean $=.68, S D=0.17 ; t=2.2, \mathrm{CI}=[0.008,0.28], p=0.038$ two sample t-test, no correction for multiple comparisons). A similar pattern was observed when sorting based on the MS rate around lag 4, which corresponded to the descending part of the rebound phase (Figure 6F). Here, participants who showed lower MS rate (no rebound) showed better performance in the T2 task (high MS rate group: mean T2 performance $=.67, S D=1.15 ;$ low $\mathrm{MS}$ rate group: mean $=0.83, S D=0.13 ; \mathrm{t}=-2.55$ $\mathrm{CI}=[-.29,-0.3], p=0.019)$. Notably, participants which showed a reduced rebound phase also showed a reduced suppressive phase. This was in contrast to what was observed while sorting participants based on the suppressive phase where both groups showed some level of rebound. Thus, while this analysis points towards the importance of the suppressive phase in determining T2 performance, the contribution of the rebound phase was less clear.

\section{Microsaccade rate signature correlates with attentional blink}

To further examine the relationship between MS rate suppression and T2 performance, pointed to in the last section, we directly plotted individual participant's lag 2 T2 performance as a function of MS rate during the lag 2 presentation time (Figure 7A; see also Figure 6D). The two factors were significantly positively correlated $\left(R^{2}=0.31, p<0.01\right)$ implying that participants who showed the largest MS rate suppression after T1 presentation also showed the largest $\mathrm{AB}$ effect. The previous analysis also pointed towards a contribution of the descending part of the rebound phase, therefore we also examined the correlation between the MS rate around lag 4 (0.47 to 0.6 s after T1 onset) and lag 2 (0.2 to $0.33 \mathrm{~s}$ after $\mathrm{T} 1$ onset) $\mathrm{T} 2$ performance (Figure 7B; see also Figure $6 \mathrm{~F}$ ). We found a significant, yet weaker negative correlation between the two factors $\left(R^{2}=0.19, p<\right.$ 0.05). We also found a significant correlation between the size of the suppressive phase and the rebound phase $\left(R^{2}=0.36, p<0.005\right)$. 

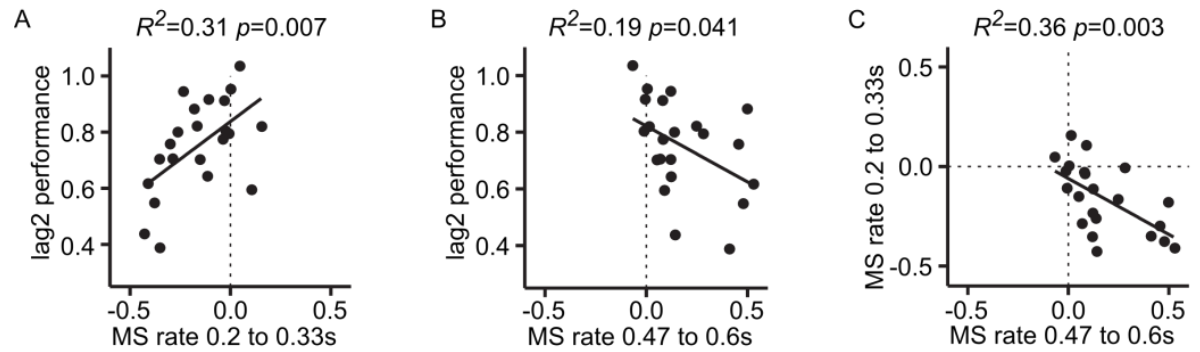

Figure 7. Lag 2 T2 performance correlates with MS rate suppression $(\boldsymbol{A})$ and rebound $(\boldsymbol{B})$ of MS rate signature. MS rate suppression correlates with MS rate rebound $(\boldsymbol{C})$. $\boldsymbol{A}$, Each point represents the MS rate (x axis) of one subject averaged from 0.2 to $0.33 \mathrm{~s}$ after $\mathrm{T} 1$ presentation (around lag 2 T2 presentation time) and lag 2 performance normalized by lag 7 performance (y axis). $\boldsymbol{B}$, The same but for MS rate 0.47 to $0.6 \mathrm{~s}$ after T1 presentation (around lag 4 presentation time). $\boldsymbol{C}$, Each point represents MS rate around the time of lag 2 presentation (y axis) and MS rate around the time of lag 4 presentation time (x axis).

\section{Microsaccade rate signature correlates with perceptual performance}

To gain further insight into the relationship between MS rate changes around the time of T1 presentation and the participants' performance in the task we extended the correlation analysis shown in Figure 7 using a sliding time window (Figure 8). At each position of the window (133ms, 10ms steps) we calculated the correlation between each participant's MS rate and their T2 performance. In addition to examining their performance at lag 2 we also examined their performance at all other T2 lags and with T1 performance. The correlation between lag 2 T2 performance and suppression of MS rate, indicated by positive slope values, was prominent for the period around the time of lag 2 presentation $(0.27 \mathrm{~s})$. The correlation between lag 2 performance and MS rate rebound, which was shown in Figure 7, was evident also in this analysis by relatively high $\mathrm{R}^{2}$ values and negative slope values, but did not reach significance using permutation testing. Interestingly the performance at lags 3, 4 and 5 was correlated with the MS rate at or just before T1 presentation where negative slope values indicated that higher MS rate corresponded with poorer performance. 


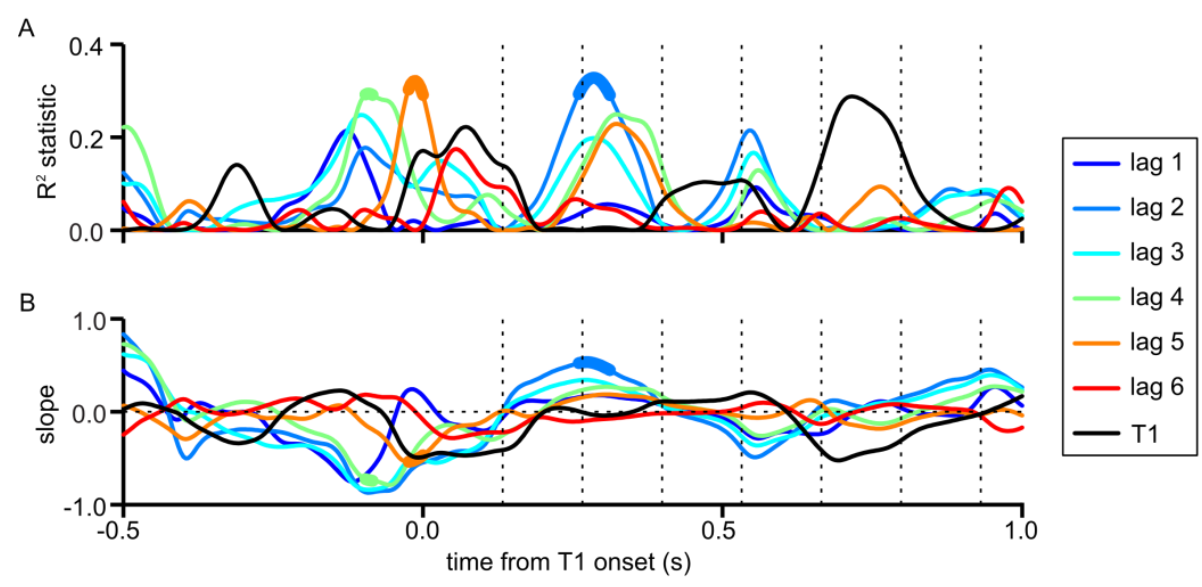

Figure 8. Sliding window (133ms width, 10ms steps) correlation of relative MS rate against task performance for $\mathrm{T} 1$ and for $\mathrm{T} 2$ in each lag (line colors). At each window position we calculated $R^{2}(\boldsymbol{A})$ and regression slope $(\boldsymbol{B})$. Positive slopes imply poor performance among subjects who showed the low MS rate. Periods where the correlation was significant $(p<$ $0.01)$ are highlighted by thicker lines in both panels. Vertical dashed lines indicate T2 onset times.

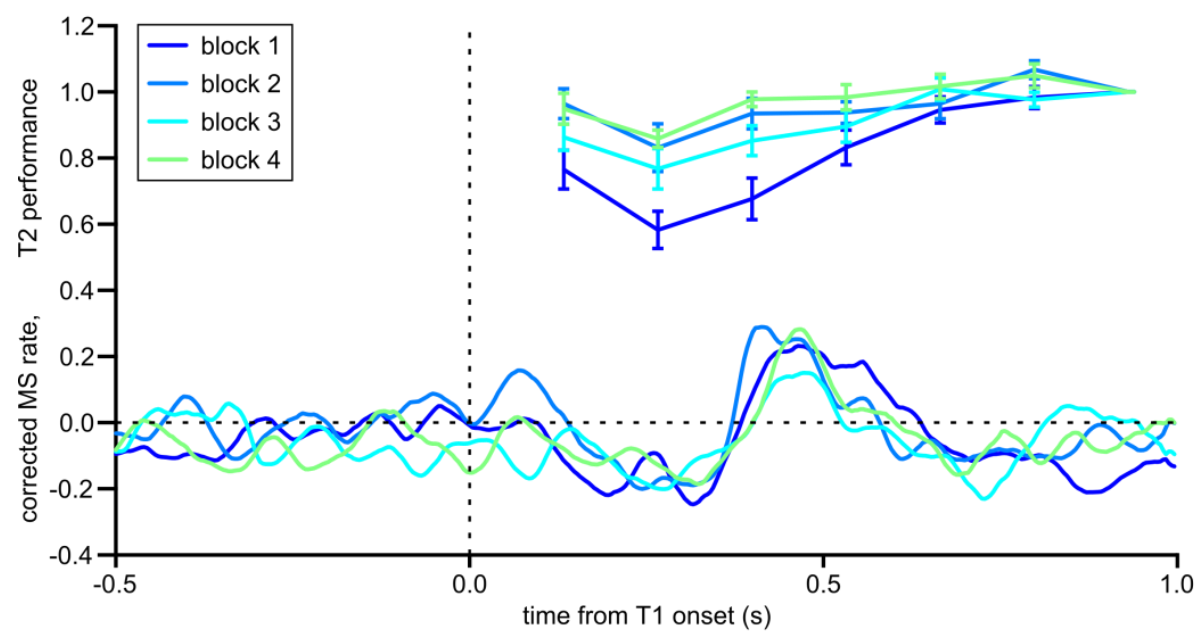

Figure 9. Task performance and MS behavior changed through the session. Data are displayed as in Figure 4, data from each block are displayed separately (colors). 


\section{Performance change through the test session}

The test session was divided into four blocks of 90 trials (10 trials per condition) with a short rest in between. For further insight we examined the data from each block separately (Figure 9). Remarkably, the AB effect reduced dramatically between the first and second block. The difference in lag 2 performance between block was significant (repeated measures ANOVA, $F(3,62)=6.01$, p <0.005). Mean MS rate during the period of the trial when targets presented (i.e. $1120 \mathrm{~ms}$ to $2730 \mathrm{~ms}$ after stimulus onset, see Figure 2) did not change significantly over the session (repeated measures ANOVA, $F(3,62)=1.92, p=0.14$ ).

\section{Microsaccade rate signature correlates with perceptual performance in expanded analysis}

The previous analysis pointed to changes in behavior through the session. We asked the question what impact these changes would have on our results. We therefore repeated the sliding window analysis but took data from each of the four blocks as a separate data point for the correlation (Figure 10). The results largely matched the earlier analysis with blocks combined, however the correlations between MS rate and lag 2 performance and with T1 performance were somewhat more prominent, while other correlations became less so. Thus, poor lag 2 T2 performance (i.e. the $\mathrm{AB}$ effect) was correlated with a suppression of MS rate, indicated by a positive slope, at around $300 \mathrm{~ms}$ after T1 and with an increase in MS rate at around $550 \mathrm{~ms}$ after T1. The correlation between lag $2 \mathrm{~T} 2$ performance and MS rate rebound was significant in this analysis shown by a negative slope. In this analysis a third period of interest also became prominent at around 930ms after T1, where a suppression of MS rate also correlated with poor lag $2 \mathrm{~T} 2$ performance. High performance in the T1 task was associated with low MS rate immediately after the T1 presentation time and with low MS rate 670 to $800 \mathrm{~ms}$ after the T1 presentation time. Interestingly, the time interval between the two periods of interest with respect to T1 performance closely matched the time interval between the first and third periods of interest with respect to lag $2 \mathrm{~T} 2$ performance. There was furthermore an intermediate period where MS suppression correlated weakly (not significantly) with T1 performance. If these three periods are taken into account it can be seen that the temporal pattern of MS rate to T1 performance correlation closely matches the pattern with respect to 
lag 2 T2 performance although the slopes are reversed. These three periods occurred at an interval of close to $.25 \mathrm{~s}$ apart, or $4 \mathrm{~Hz}$. The pattern is reminiscent of the recent observation of theta-rhythmic switching of attention towards and away from attended stimuli (Landau \& Fries, 2012).

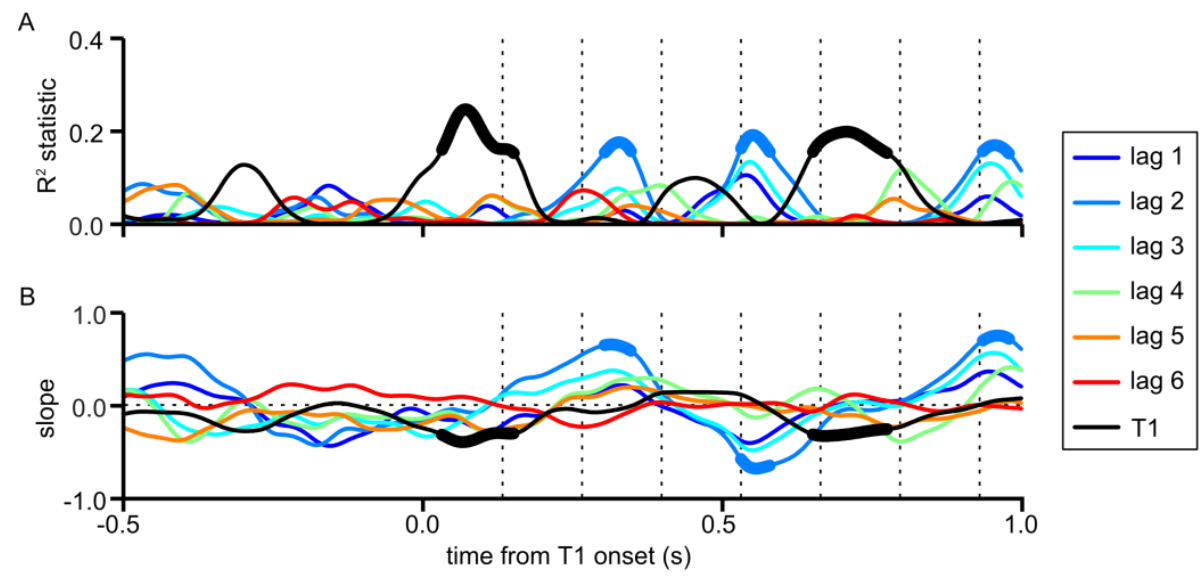

Figure 10. Sliding window (133ms width, 10ms steps) correlation of relative MS rate against task performance for each lag and T1 (line colors). Data from separate runs was taken as separate data points in the correlation analysis. At each window position we calculated the regression slope, $R^{2}$ and significance $(\boldsymbol{A})$. The correlation between lag 2 performance (light blue line) and MS rate in the period just after lag 2 presentation time was noticeably strong, with a high positive slope $(\boldsymbol{B})$. T1 performance also correlated with MS rate in the period just after $\mathrm{T} 1$ presentation and the period just after lag 5 with a shallow negative slope implying a high MS rate corresponded to poor T1 performance. Vertical dashed lines indicate T2 onset times.

\section{Summary}

We found that MS rate rapidly varied following T1 onset, following a pattern of initial suppression followed by rebound and further suppression (Figure 11, blue line and shading). Moreover, we found that changes in MS rate were significantly correlated to the magnitude of the $\mathrm{AB}$ effect at three time periods (Figure 11, red highlighting). 


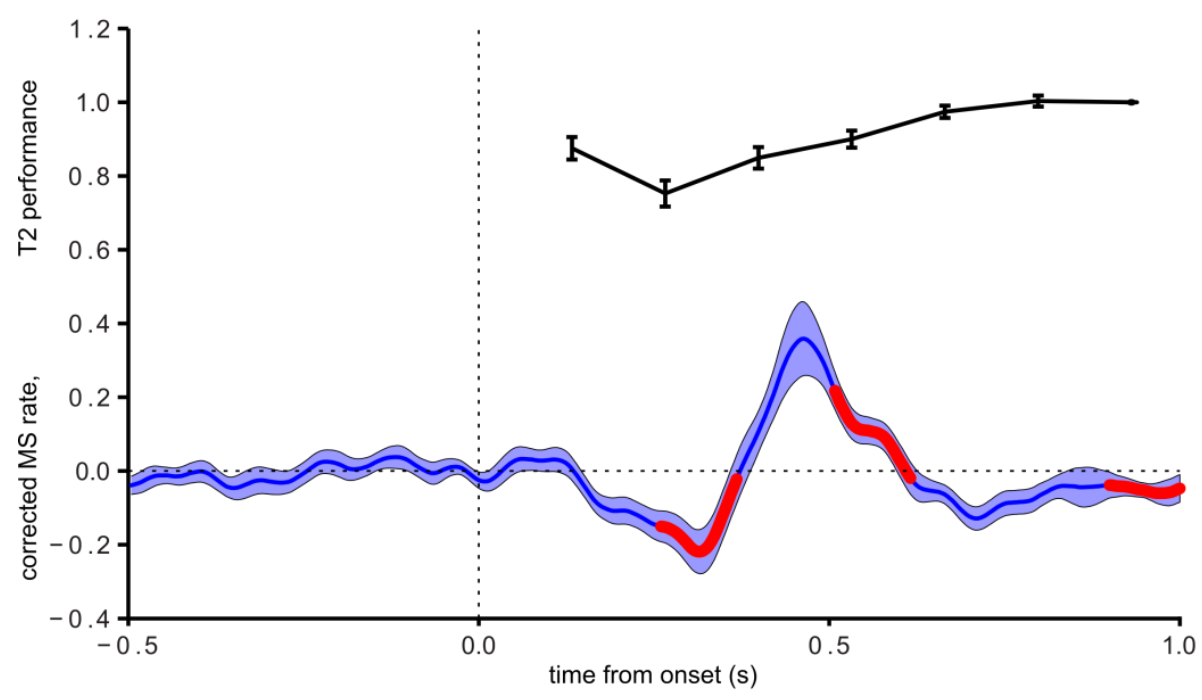

Figure 11. Summary of observed effects. Blue line and shading shows changes in MS rate after T1 onset. Red highlighting shows time periods where MS rate was significantly correlated with T2 performance at lag 2. Black solid lines show behavioral performance in T2 task (normalized by performance at lag 7). 


\section{Discussion}

We investigated the relationship between temporal variation in attention and in microsaccade rate in the $\mathrm{AB}$ paradigm. We had expected that anticipation of $\mathrm{T} 1$ would be associated with microsaccade suppression and that after successful detection MS rate would rebound to levels observed during inattentive states. We hypothesized that this rebound would coincide with presentation of the second target around $200 \mathrm{~ms}$ and thus result in impaired detection performance. To the contrary we found that the period of time in which T2 processing was impaired was associated with a suppression of microsaccade rate. Hence, the unavailability of attention for $\mathrm{T} 2$ after processing of $\mathrm{T} 1$, hypothesized to underlie the $\mathrm{AB}$ for $\mathrm{T} 2$, correlates with a reduction in microsaccade rate.

The pattern of MS suppression and rebound reported here are similar to the pattern reported in response to a visual oddball in a RSVP task (Valsecchi et al., 2007; Valsecchi et al., 2009). In our task the T1 was colored differently from the distractors and would therefore be described as an oddball. Moreover, attention to the oddball has been shown to enhance the MS rate signature. In the AB paradigm the more attention that is deployed for T1, either because it is more salient, more task relevant or requires more encoding into working memory, the greater the blink effect. Similarly drawing attention away from T1, either by explicitly instructing participants to ignore it (Raymond, Shapiro, \& Arnell, 1992), by cuing a distractor prior to T2 (e.g., Nieuwenstein, 2006) or by including distracting tasks, tends to reduce the T2 deficit, though notable exceptions are reported (e.g., Stein, Zwickel, Kitzmantel, Ritter, \& Schneider, 2010; Van der Burg, Nieuwenstein, Theeuwes, \& Olivers, 2013). These results are fully in line with our current findings; it can be expected that an unattended T1 (oddball) will result in a reduced amplitude of the MS rate signature, in line with a reduced $A B$.

The question of whether the $\mathrm{AB}$ occurs for cross modal paradigms has attracted considerable interest. While there exists many conflicting reports and the issue is not fully resolved, there are sufficient reports to show that under certain conditions an auditory or tactile T1 can induce a visual AB effect (Jolicoeur, 1999; Soto-Faraco et al., 2002). In this regard it is therefore interesting to note that auditory (Rolfs et al., 2005; Widmann, Engbert, \& Schröger, 2014) and tactile (Soto-Faraco et al., 2002) 
stimuli are widely reported to mediate an MS rate signature. Further research could explore the dependencies of the MS rate signature and of the AB effect in crossmodal designs. Our current results suggest that $\mathrm{AB}$ will be absent in tasks which do not yield a suppression of MS following the T1.

\section{Fading as an alternative explanation of the attentional blink effect}

We found that failure to identify the T2 was associated with a reduction in MS rate. Microsaccades are thought to play a critical role in visual processing by preventing adaptation and fading of static retinal images. For example some reports suggest fading in very brief time periods (as fast as 80ms) in case of perfect retinal stabilization (Coppola \& Purves, 1996; De Weerd, 2006; Ditchburn \& Ginsborg, 1953; Gerrits, De Haan, \& Vendrik, 1966; Riggs \& Ratliff, 1952; Riggs, Ratliff, Cornsweet, \& Cornsweet, 1953). One plausible account of our data could therefore be that suppression of MS following the T1 leads to degradation of the T2 input through fading. In line with this account one could argue that the rise in T2 performance at later lags occurred, because the rebound in MS rate eliminated any fading.

While fading remains a possible account for the attentional blink, its plausibility depends on how much time fading would take in our experimental design. The period of MS suppression in our study started $160 \mathrm{~ms}$ after the T1 onset and ended 344ms after, thus lasting less than 200ms. Costela et al. (2013) showed that typically periods of MS suppression of more than $1000 \mathrm{~ms}$ were observed before fading set in. Similarly, Cui et al. (2009) showed that the addition of a surround to a target yielded a suppression of MS rate and that targets were invisible on trials where this suppression lasted more than $700 \mathrm{~ms}$. Crucially, in that study MS suppression for up to $400 \mathrm{~ms}$ was observed in trials where the target remained visible. These data suggest that the period of MS suppression observed in the current study may have been too short to allow fading to take place to the extent that it could account for suppressed T2 performance. In addition, it has been suggested that for foveal stimuli such as ours drift and tremor are sufficient to prevent fading as receptive fields are so small (Martinez-Conde, Macknik, \& Hubel, 2004).

Yet another alternative is that the blink effect could be attributed to flicker adaptation. Flickering peripheral stimuli have been shown to fade faster with 
increasing eccentricity and high flicker frequency such as used in our experiment (Schieting \& Spillmann, 1987). However, considering the foveal RSVP stream, and the lag-dependent variation in behavioral performance, it seems excluded that the attentional blink effect is due to flicker adaptation.

\section{Microsaccades as an index for measuring attentional deployment}

Several studies have shown that states of attention are associated with a lowering of MS rate (Engbert \& Kliegl, 2003; Gowen et al., 2007; Kohama \& Usui, 2002). Several authors additionally show that, in their task, stimuli presented at the moment of a microsaccade were poorly perceived (Beeler, 1967; Godlove \& Schall, 2014; Hafed, Lovejoy, \& Krauzlis, 2011; Herrington et al., 2009), thus suppressing MS can be an adaptive strategy for optimized performance. We show that the moment of strongest MS suppression occurred during the period immediately after the T1, a period which has been extensively described as one characterized by limited attentional resources. This would appear to speak against the suggestion that low MS rate is always reliably associated with high levels of attention.

Alternatively, attention may be described as fully deployed to the (internal) processing of the T1 during the presentation of unseen T2s. This account would be compatible with the assertion that low MS rate corresponds to high states of attention (Chun, Golomb, \& Turk-Browne, 2011; Griffin \& Nobre, 2003). Without further research into this topic, the utility of an index which cannot distinguish between deployment and withdrawal of attention to current stimuli seems limited. Recent debate has focused on MS direction as an index for the spatial location of attentional deployment (Betta \& Turatto, 2006; Engbert \& Kliegl, 2003; Hafed \& Clark, 2002; Laubrock et al., 2005). In our experiments the stimuli were presented foveally making it difficult to predict what a bias in MS direction would indicate. More research will be required to elucidate how MS direction is influenced by temporal attention.

\section{Attention mediates microsaccade rate}

We show that MSs are suppressed during the period when capacity for processing new stimuli is limited and rebounds together with performance. One account for this behavior is that the processing of the $\mathrm{T} 1$ also limits the processing capacity required 
to make new MS. This is in line with the idea that microsaccades are involved in active sensation, i.e. the active sampling of the environment for (new) visual information, similarly to whisking in rodents. The observed suppression of MS rate would then be related to less effective programming of MSs and less effective allocation of attention would follow.

Corbetta and colleagues (1998) have shown that the brain regions involved in attention on the one hand and voluntary eye-movements on the other hand largely correspond. This functional anatomical overlap is seen for parietal and frontal regions (Corbetta et al., 1998). The generation of saccades includes cortical areas (dorsolateral prefrontal cortex, frontal eye field (FEF), supplementary eye field, lateral intraparietal cortex (LIP)), basal ganglia structures (caudate nucleus, substantia nigra pars reticulata), brainstem structures (superior colliculus (SC), reticular formation), and structures in the cerebellum (oculomotor vermis, fastigial oculomotor region) as well documented elsewhere (Martinez-Conde, Otero-Millan, \& Macknik, 2013). Microsaccades are thought to have the same generator as saccades (Rolfs et al., 2008b) though the exact mechanisms are not yet understood (for a review, see Martinez-Conde et al., 2013).

Interestingly, microstimulation of FEF (Moore \& Fallah, 2001) and SC (Cavanaugh, Alvarez, \& Wurtz, 2006; Müller, Philiastides, \& Newsome, 2005) leads to spatially specific attentional enhancement in the absence of eye movements. Similarly, microstimulation of LIP amplifies attention (Cutrell \& Marrocco, 2002), however lacking spatial specificity. Beside this neurophysiological data, there is also ample evidence in humans that the locus of attention and MS direction are linked for both stimulus-triggered (Hafed \& Clark, 2002; Hafed et al., 2011; Laubrock et al., 2005; Laubrock et al., 2007; Laubrock et al., 2010) and spontaneous MSs (YuvalGreenberg et al., 2014). It has also been suggested that the direction of early MSs indicates attention shifts after a cue, whereas sustained lateralization of microsaccade related potentials (mSRP) indicates the locus of sustained attention (Meyberg, Werkle-Bergner, Sommer, \& Dimigen, 2015). Of course, one needs to be aware that, given the variety of SC inputs, it seems plausible that several processes influence the relationship between MSs and covert attention (Laubrock et al., 2010). A model aimed at explaining the occurrence of eye movements posits that fixation is 
achieved when retinotopically organized visuomotor maps in SC are kept in balance. However, once this balance is shifted, as might happen through attention, microsaccades might be initiated (Hafed, Goffart, \& Krauzlis, 2009; Hafed, Lovejoy, \& Krauzlis, 2013). A recent study moreover showed that spatially specific SC inactivation resulted in changed MS direction but not rate (Hafed et al., 2013) which indicates that the SC is at least partially accountable for the link between covert attention and microsaccades.

There have been attempts to link the $\mathrm{AB}$ phenomenon to eye movements. $\mathrm{A}$ study investigating the role of dopamine in the $A B$ showed that that spontaneous eyeblink rate, a marker for dopaminergic functioning, predicts the size of $\mathrm{AB}$ (Colzato, Slagter, Spapé, \& Hommel, 2008). Though eye blinks are merely used as a marker in this study and blinks are not eye movements, the AB effect within and across fixation periods has also been investigated (Kamienkowski, Navajas, \& Sigman, 2012). In contrast to the present study, which focused on microsaccades, they explored in how far saccades affect the AB. More specifically, stimulussaccade asynchronies (SSA) was calculated and showed that the AB was diminished when T2 was presented far in time from saccades, and more prominent when presented close in time to a saccade. In another study, it has been shown that attentional allocation (sustained attention, spatial attention transient, temporal attention transient (i.e. attentional blink)) on a peripheral RSVP stream also influences microsaccades rate and direction (Pastukhov, Vonau, Stonkute, \& Braun, 2013). The microsaccade rate signature was increased for successful compared to failed target detection. However, the timing of this microsaccade rate signature was very different from the one observed in our study. Successful target detection led to decreased microsaccade rate around $500 \mathrm{~ms}$ and a rebound at $1000 \mathrm{~ms}$, for missed targets the microsaccade rate was suppressed at 500ms and $1500 \mathrm{~ms}$ and showed a rebound at 2000ms. Our modulation happens much earlier at around 250ms for the suppression and $500 \mathrm{~ms}$ for the rebound. To investigate the involvement of posterior parietal cortex in the $\mathrm{AB}$, repetitive transcranial stimulation (rTMS) has been applied to right posterior parietal cortex (Cooper, Humphreys, Hulleman, Praamstra, $\&$ Georgeson, 2004). Stimulation reduced the magnitude of the attentional blink, with no such effect when stimulating a control site. Importantly, T1 detection 
performance was not affected by rTMS. These results thus confirm that posterior parietal cortex plays an important role in the attentional blink. If one argues that the $\mathrm{AB}$ is the result of prolonged capturing of attention by $\mathrm{T} 1$ presentation, then one possible explanation for these results is that rTMS disrupted posterior parietal cortex functioning and therefore the attentional engagement to T1. Given the stimulation location it seems likely that LIP (associated with both eye movements and attention) was affected by rTMS. It thus is possible that microsaccades were affected by TMS stimulation and that this was the cause of the reduced attentional blink. One might argue that during the task participants fixate and thus LIP is suppressing (micro)saccades. TMS then would disrupt this process, thereby increasing the MS rate and thus weaken the attentional blink. Taken together, these studies support the notion that eye movements may play a role in the $\mathrm{AB}$ and further research should try to specify this relationship.

Saccades and microsaccades have been shown to occur with intersaccadic intervals of 200ms (Otero-Millan, Troncoso, Macknik, Serrano-Pedraza, \& Martinez-Conde, 2008b) which corresponds to a theta rhythm, and have been suggested to reflect an ongoing endogenous rhythm that can be reset by stimulus events (Landau \& Fries, 2012). Interestingly, when correlating MS rate with T1 task performance and T2 task performance at lag 2 using a sliding-window approach a similar temporal relationship was seen, suggesting that our modulations in MS rate occur at the rhythm of microsaccade generation. This would be in line with a theta MS rhythm which is linked to attentional switching and is reset by T1 stimulus onset. Resetting the attention/MS rhythm following T1 presentation inevitably leads to the lag 2 time interval aligning with a withdrawal phase of this $4 \mathrm{~Hz}$ rhythm.

\section{Conclusion}

Contrary to our hypotheses we found that the microsaccade rate is suppressed during the time around $250 \mathrm{~ms}$ after T1 presentation, which is the typical time lag at which the $\mathrm{AB}$ effect is observed. At around $500 \mathrm{~ms}$ the MS rate rebounds and then returns to baseline level. Interestingly, this microsaccade rate signature is increased for participants showing a stronger $\mathrm{AB}$ effect. Our results are in line with studies emphasizing the close link between attentional processes and microsaccades. 


\section{Acknowledgements}

GL was supported by a grant from the Netherlands Organization for Scientific Research to the FPN Graduate School for Cognitive and Clinical Neuroscience (NWO, 022.001.036). PDW was supported by a grant from the Netherlands Organization for Scientific Research (NWO VICI grant, 453.04.002). 


\section{References}

Beeler, G. W., Jr. (1967). Visual threshold changes resulting from spontaneous saccadic eye movements. Vision Research, 7(9), 769-775.

Betta, E., Galfano, G., \& Turatto, M. (2007). Microsaccadic response during inhibition of return in a target-target paradigm. Vision Research, 47(3), 428-436.

Betta, E., \& Turatto, M. (2006). Are you ready? I can tell by looking at your microsaccades. Neuroreport, 17(10), 1001-1004.

Bonneh, Y. S., Donner, T. H., Sagi, D., Fried, M., Cooperman, A., Heeger, D. J., \& Arieli, A. (2010). Motion-induced blindness and microsaccades: Cause and effect. Journal of Vision, 10(14), 22.

Bosman, C. A., Womelsdorf, T., Desimone, R., \& Fries, P. (2009). A microsaccadic rhythm modulates gamma-band synchronization and behavior. The Journal of Neuroscience, 29(30), 9471-9480.

Bridgeman, B., \& Palca, J. (1980). The role of microsaccades in high acuity observational tasks. Vision Research, 20(9), 813-817.

Cavanaugh, J., Alvarez, B. D., \& Wurtz, R. H. (2006). Enhanced performance with brain stimulation: Attentional shift or visual cue? The Journal of Neuroscience, 26(44), 11347-11358.

Chun, M. M., Golomb, J. D., \& Turk-Browne, N. B. (2011). A taxonomy of external and internal attention. Annual Review of Psychology, 62, 73-101.

Colzato, L. S., Slagter, H. A., Spapé, M., \& Hommel, B. (2008). Blinks of the eye predict blinks of the mind. Neuropsychologia, 46(13), 3179-3183.

Cooper, A. C., Humphreys, G. W., Hulleman, J., Praamstra, P., \& Georgeson, M. (2004). Transcranial magnetic stimulation to right parietal cortex modifies the attentional blink. Experimental Brain Research, 155(1), 24-29.

Coppola, D., \& Purves, D. (1996). The extraordinarily rapid disappearance of entopic images. Proceedings of the National Academy of Sciences, 93(15), 80018004.

Corbetta, M., Akbudak, E., Conturo, T. E., Snyder, A. Z., Ollinger, J. M., Drury, H. A., . . Shulman, G. L. (1998). A common network of functional areas for attention and eye movements. Neuron, 21(4), 761-773.

Costela, F. M., McCamy, M. B., Macknik, S. L., Otero-Millan, J., \& MartinezConde, S. (2013). Microsaccades restore the visibility of minute foveal targets. PeerJ, 1, e119. 
Cui, J., Wilke, M., Logothetis, N. K., Leopold, D. A., \& Liang, H. (2009). Visibility states modulate microsaccade rate and direction. Vision Research, 49(2), 228-236.

Cui, J., Wilke, M., Logothetis, N. K., Leopold, D. A., \& Liang, H. (2009). Visibility states modulate microsaccade rate and direction. Vision Research, 49(2), 228-236.

Cutrell, E., \& Marrocco, R. (2002). Electrical microstimulation of primate posterior parietal cortex initiates orienting and alerting components of covert attention. Experimental Brain Research, 144(1), 103-113.

Desimone, R., \& Duncan, J. (1995). Neural mechanisms of selective visual attention. Annual review of neuroscience, 18(1), 193-222.

Dux, P. E., \& Marois, R. (2009). The attentional blink: A review of data and theory. Attention, Perception, \& Psychophysics, 71(8), 1683-1700.

Engbert, R. (2006). Microsaccades: A microcosm for research on oculomotor control, attention, and visual perception. Progress in Brain Research, 154, 177-192.

Engbert, R., \& Kliegl, R. (2003). Microsaccades uncover the orientation of covert attention. Vision Research, 43(9), 1035-1045.

Galfano, G., Betta, E., \& Turatto, M. (2004). Inhibition of return in microsaccades. Experimental Brain Research, 159(3), 400-404.

Godlove, D. C., \& Schall, J. D. (2014). Microsaccade production during saccade cancelation in a stop-signal task. Vision Research.

Gonzalez, E. G., Wong, A. M., Niechwiej-Szwedo, E., Tarita-Nistor, L., \& Steinbach, M. J. (2012). Eye position stability in amblyopia and in normal binocular vision. Investigative Ophthalmology \& Visual Science, 53(9), 5386-5394.

Gowen, E., Abadi, R. V., \& Poliakoff, E. (2005). Paying attention to saccadic intrusions. Brain Res Cogn Brain Res, 25(3), 810-825.

Gowen, E., Abadi, R. V., Poliakoff, E., Hansen, P. C., \& Miall, R. C. (2007). Modulation of saccadic intrusions by exogenous and endogenous attention. Brain Research, 1141, 154-167.

Griffin, I. C., \& Nobre, A. C. (2003). Orienting attention to locations in internal representations. Journal of Cognitive Neuroscience, 15(8), 1176-1194.

Hafed, Z. M. (2013). Alteration of visual perception prior to microsaccades. Neuron, 77(4), 775-786.

Hafed, Z. M., \& Clark, J. J. (2002). Microsaccades as an overt measure of covert attention shifts. Vision Research, 42(22), 2533-2545. 
Hafed, Z. M., Goffart, L., \& Krauzlis, R. J. (2009). A neural mechanism for microsaccade generation in the primate superior colliculus. Science, 323(5916), 940943.

Hafed, Z. M., \& Ignashchenkova, A. (2013). On the dissociation between microsaccade rate and direction after peripheral cues: Microsaccadic inhibition revisited. The Journal of Neuroscience, 33(41), 16220-16235.

Hafed, Z. M., Lovejoy, L. P., \& Krauzlis, R. J. (2011). Modulation of microsaccades in monkey during a covert visual attention task. The Journal of Neuroscience, 31(43), 15219-15230.

Hafed, Z. M., Lovejoy, L. P., \& Krauzlis, R. J. (2013). Superior colliculus inactivation alters the relationship between covert visual attention and microsaccades. European Journal of Neuroscience, 37(7), 1169-1181.

Hass, C. A., \& Horwitz, G. D. (2011). Effects of microsaccades on contrast detection and v1 responses in macaques. Journal of Vision, 11(3), 1-17.

Herrington, T. M., Masse, N. Y., Hachmeh, K. J., Smith, J. E. T., Assad, J. A., \& Cook, E. P. (2009). The effect of microsaccades on the correlation between neural activity and behavior in middle temporal, ventral intraparietal, and lateral intraparietal areas. The Journal of Neuroscience, 29(18), 5793-5805.

Horowitz, T. S., Fine, E. M., Fencsik, D. E., Yurgenson, S., \& Wolfe, J. M. (2007). Fixational eye movements are not an index of covert attention. Psychological Science, 18(4), 356-363.

Hsieh, P. J., \& Tse, P. U. (2009a). Microsaccade rate varies with subjective visibility during motion-induced blindness. PLoS One, 4(4), e5163.

Hsieh, P. J., \& Tse, P. U. (2009b). Motion fading and the motion aftereffect share a common process of neural adaptation. Attention, Perception, \& Psychophysics, 71(4), 724-733.

Jolicoeur, P. (1999). Restricted attentional capacity between sensory modalities. Psychonomic Bulletin \& Review, 6(1), 87-92.

Kamienkowski, J. E., Navajas, J., \& Sigman, M. (2012). Eye movements blink the attentional blink. Journal of Experimental Psychology: Human Perception and Performance, 38(3), 555.

Kohama, T., Endoh, S., \& Yoshida, H. (2013). Persistent inhibition of microsaccades caused by attentional concentration. Perception, 42(ECVP Abstract Supplement), 38-38. 
Kohama, T., \& Usui, S. (2002). Attentional effects on microsaccadic eye movements. Cahiers De Psychologie Cognitive-Current Psychology of Cognition, 21(4-5), 377-395.

Landau, A. N., \& Fries, P. (2012). Attention samples stimuli rhythmically. Current Biology, 22(11), 1000-1004.

Laubrock, J., Engbert, R., \& Kliegl, R. (2005). Microsaccade dynamics during covert attention. Vision Research, 45(6), 721-730.

Laubrock, J., Engbert, R., Rolfs, M., \& Kliegl, R. (2007). Microsaccades are an index of covert attention: Commentary on Horowitz, Fine, Fencsik, Yurgenson, and Wolfe (2007). Psychological Science, 18(4), 364-366; discussion 367-368.

Laubrock, J., Kliegl, R., Rolfs, M., \& Engbert, R. (2010). When do microsaccades follow spatial attention? Attention, Perception, \& Psychophysics, 72(3), 683-694.

Lehky, S. R. (2009). Decoding poisson spike trains by gaussian filtering. Neural Computation, 22(5), 1245-1271.

Litvak, V., Mattout, J., Kiebel, S., Phillips, C., Henson, R., Kilner, J., . . Flandin, G. (2011). Eeg and meg data analysis in spm8. Computational intelligence and neuroscience, 2011.

Maij, F., Matziridi, M., Smeets, J. B., \& Brenner, E. (2012). Luminance contrast in the background makes flashes harder to detect during saccades. Vision Research, 60, 22-27.

Maris, E., \& Oostenveld, R. (2007). Nonparametric statistical testing of eeg- and meg-data. J Neurosci Methods, 164(1), 177-190.

Martinez-Conde, S., Macknik, S. L., \& Hubel, D. H. (2004). The role of fixational eye movements in visual perception. Nature Reviews Neuroscience, 5(3), 229-240.

Martinez-Conde, S., Macknik, S. L., Troncoso, X. G., \& Dyar, T. A. (2006). Microsaccades counteract visual fading during fixation. Neuron, 49(2), 297-305.

Martinez-Conde, S., Otero-Millan, J., \& Macknik, S. L. (2013). The impact of microsaccades on vision: Towards a unified theory of saccadic function. Nature Reviews Neuroscience, 14(2), 83-96.

McCamy, M. B., Macknik, S. L., \& Martinez-Conde, S. (2014). Different fixational eye movements mediate the prevention and the reversal of visual fading. The Journal of Physiology, 592(Pt 19), 4381-4394.

Meyberg, S., Werkle-Bergner, M., Sommer, W., \& Dimigen, O. (2015). Microsaccade-related brain potentials signal the focus of visuospatial attention. NeuroImage, 104(0), 79-88. 
Møller, F., Laursen, M., Tygesen, J., \& Sjølie, A. (2002). Binocular quantification and characterization of microsaccades. Graefe's archive for clinical and experimental ophthalmology, 240(9), 765-770.

Moore, T., \& Fallah, M. (2001). Control of eye movements and spatial attention. Proceedings of the National Academy of Sciences, 98(3), 1273-1276.

Müller, J. R., Philiastides, M. G., \& Newsome, W. T. (2005). Microstimulation of the superior colliculus focuses attention without moving the eyes. Proceedings of the National Academy of Sciences of the United States of America, 102(3), 524-529.

Nieuwenstein, M. R. (2006). Top-down controlled, delayed selection in the attentional blink. Journal of Experimental Psychology: Human Perception and Performance, 32(4), 973-985.

Otero-Millan, J., Troncoso, X. G., Macknik, S. L., Serrano-Pedraza, I., \& MartinezConde, S. (2008a). Saccades and microsaccades during visual fixation, exploration, and search: Foundations for a common saccadic generator. $J$ Vis, 8(14), 21 21-18.

Otero-Millan, J., Troncoso, X. G., Macknik, S. L., Serrano-Pedraza, I., \& MartinezConde, S. (2008b). Saccades and microsaccades during visual fixation, exploration, and search: Foundations for a common saccadic generator. Journal of Vision, 8(14), 21 21-18.

Pastukhov, A., \& Braun, J. (2010). Rare but precious: Microsaccades are highly informative about attentional allocation. Vision Research, 50(12), 1173-1184.

Pastukhov, A., Vonau, V., Stonkute, S., \& Braun, J. (2013). Spatial and temporal attention revealed by microsaccades. Vision Research, 85(0), 45-57.

Raymond, J. E., Shapiro, K. L., \& Arnell, K. M. (1992). Temporary suppression of visual processing in an rsvp task: An attentional blink? Journal of Experimental Psychology: Human Perception and Performance, 18(3), 849.

Riggs, L. A., Ratliff, F., Cornsweet, J. C., \& Cornsweet, T. N. (1953). The disappearance of steadily fixated visual test objects. Journal of the Optical Society of America, 43(6), 495-501.

Rolfs, M. (2009). Microsaccades: Small steps on a long way. Vision Research, 49(20), 2415-2441.

Rolfs, M., Engbert, R., \& Kliegl, R. (2004). Microsaccade orientation supports attentional enhancement opposite a peripheral cue: Commentary on tse, sheinberg, and logothetis (2003). Psychol Sci, 15(10), 705-707; author reply 708-710. 
Rolfs, M., Engbert, R., \& Kliegl, R. (2005). Crossmodal coupling of oculomotor control and spatial attention in vision and audition. Experimental Brain Research, 166(3-4), 427-439.

Rolfs, M., Kliegl, R., \& Engbert, R. (2008a). Toward a model of microsaccade generation: The case of microsaccadic inhibition. $J$ Vis, 8(11), 5 1-23.

Rolfs, M., Kliegl, R., \& Engbert, R. (2008b). Toward a model of microsaccade generation: The case of microsaccadic inhibition. Journal of Vision, 8(11), 5 1-23.

Rucci, M., \& Desbordes, G. (2003). Contributions of fixational eye movements to the discrimination of briefly presented stimuli. Journal of Vision, 3(11), 852-864.

Schütz, A. C., Braun, D. I., \& Gegenfurtner, K. R. (2009). Object recognition during foveating eye movements. Vision Research, 49(18), 2241-2253.

Soto-Faraco, S., Spence, C., Fairbank, K., Kingstone, A., Hillstrom, A. P., \& Shapiro, K. (2002). A crossmodal attentional blink between vision and touch. Psychon Bull Rev, 9(4), 731-738.

Stein, T., Zwickel, J., Kitzmantel, M., Ritter, J., \& Schneider, W. X. (2010). Irrelevant words trigger an attentional blink. Experimental Psychology, 57(4), 301307.

Troncoso, X. G., Macknik, S. L., \& Martinez-Conde, S. (2008). Microsaccades counteract perceptual filling-in. Journal of Vision, 8(14), 15 11-19.

Tse, P. U. (2004). Mapping visual attention with change blindness: New directions for a new method. Cognitive Science, 28(2), 241-258.

Tse, P. U., Sheinberg, D. L., \& Logothetis, N. K. (2002). Fixational eye movements are not affected by abrupt onsets that capture attention. Vision Research, 42(13), 1663-1669.

Tse, P. U., Sheinberg, D. L., \& Logothetis, N. K. (2003). Attentional enhancement opposite a peripheral flash revealed using change blindness. Psychological Science, 14(2), 91-99.

Valsecchi, M., Betta, E., \& Turatto, M. (2007). Visual oddballs induce prolonged microsaccadic inhibition. Experimental Brain Research, 177(2), 196-208.

Valsecchi, M., Dimigen, O., Kliegl, R., Sommer, W., \& Turatto, M. (2009). Microsaccadic inhibition and p300 enhancement in a visual oddball task. Psychophysiology, 46(3), 635-644.

Valsecchi, M., \& Turatto, M. (2009). Microsaccadic responses in a bimodal oddball task. Psychological Research, 73(1), 23-33. 
Van der Burg, E., Nieuwenstein, M. R., Theeuwes, J., \& Olivers, C. N. L. (2013). Irrelevant auditory and visual events induce a visual attentional blink. Experimental Psychology, 60(2), 80-89.

Widmann, A., Engbert, R., \& Schröger, E. (2014). Microsaccadic responses indicate fast categorization of sounds: A novel approach to study auditory cognition. The Journal of Neuroscience, 34(33), 11152-11158.

Yuval-Greenberg, S., Merriam, E. P., \& Heeger, D. J. (2014). Spontaneous microsaccades reflect shifts in covert attention. The Journal of Neuroscience, 34(41), 13693-13700.

Zuber, B. L., Stark, L., \& Cook, G. (1965). Microsaccades and the velocityamplitude relationship for saccadic eye movements. Science, 150(3702), 1459-1460. 


\section{CHAPTER 7}

Summary and Conclusions 
In the present thesis we used psychophysical experiments to investigate perceptual learning and attention. More specifically, we tested hypotheses regarding the extent of the neural network contributing to orientation discrimination learning (Chapters 2 and 3), the nature of the process that leads to the stabilization of visual skill memory (Chapter 4 and 5), and the link between attention, microsaccades and the attentional blink effect (Chapter 6). These three broad questions will be discussed in the following.

\section{What are the contributions of low-level plasticity and plasticity in read-out to visual skill learning?}

We found large generalization of orientation discrimination skill, which, however, was not complete: There was limited but significant orientation and position specificity, measured as an advantage in performance when tested on the specific orientation and position used in training, compared to testing at other positions/orientations. This advantage however was much smaller than the total magnitude of learning progress over 15 sessions of orientation discrimination training. Our studies support the view that specificity is most strongly related to asymptotic learning, as only the amount of late learning was predictive of both position and orientation specificity. However, there was only weak evidence for asymmetric generalization across different test positions in our data. Overall, the psychophysical data thus confirm some important notions such as a certain degree of specificity, which is achieved during asymptotic learning, but we cannot make strong claims on the contribution of low-level plasticity (Chapter 2).

The specificity we observed for position and orientation was less strong than that reported in some other studies (e.g. Schoups, Vogels, \& Orban, 1995). There are different possible explanations for this difference, among which the use in other studies of different stimuli, test locations with identical eccentricity but varying polar angle, and various differences in task parameters. For example, Schoups et al. (1995) used more trials in total and per session, more staircases per session, and longer training. All those factors reportedly favor finding specificity (e.g., Aberg, Tartaglia, \& Herzog, 2009; Hung \& Seitz, 2014; Jeter, Dosher, Liu, \& Lu, 2010). Lowest-level theories of perceptual learning ascribe specificity to tuning changes 
occurring in low-level areas (Karni \& Bertini, 1997). Based on cortical magnification in these areas, one could expect any spread of expertise to occur more strongly towards more peripheral than to more central location relative to the trained location. An alternative view is that generalization is rule-based and occurs in a manner that is not directly driven by low-level mechanisms (Xiao et al., 2008; Zhang, Xiao, Klein, Levi, \& Yu, 2010). In this case, any spread of expertise (by enhanced read-out) should not be guided by cortical magnification. We found some weak evidence indicating a learning-induced bias for more generalization of expertise towards peripheral than towards central locations. This pattern in our data supports an involvement of low-level areas characterized by cortical magnification in skill learning and in the generalization of expertise. However, the data do not exclude a possible contribution of read-out mechanisms to generalization if through training they would target task-relevant neural populations with some (but nonperfect) specificity. It should be emphasized that the asymmetric pattern of generalization is weak, and awaits confirmation pending additional experiments. Potentially, longer training could produce more conclusive results, since Schoups et al. (1995) also reported lower orientation discrimination thresholds than observed in our study. These might reflect in part differences in stimuli, and differences in motivation in populations of participants, but also the number of trials might have contributed to this result.

In contrast, other work has shown that under certain conditions (doubletraining, i.e., a combination of feature and location training, or the introduction of a brief pre-test) complete generalization can be achieved (Xiao et al., 2008; T. Zhang et al., 2010). In such experimental designs, task relevancy and attentional demand at a training location determine whether complete, partial or no transfer is observed (Wang, Zhang, Klein, Levi, \& Yu, 2012), with higher relevancy leading to stronger transfer. Notably, these effects have been reported after seven sessions of training and the question can be asked whether double-training also would result in similar generalization if training would have been continued until reaching asymptotic performance levels. In response to that question, it has been shown that increasing the amount of training at threshold level can restore specificity in a double-training paradigm (Hung \& Seitz, 2014). These studies combined suggested to us that 
conditions inducing transfer are found predominantly in the early part of learning, whereas specificity results from late, asymptotic learning, in line with data from earlier studies (Ahissar \& Hochstein, 1996; Crist, Kapadia, Westheimer, \& Gilbert, 1997; Fahle, Edelman, \& Poggio, 1995; Karni \& Sagi, 1991; Schoups et al., 1995)

To substantiate this hypothesis, we decided to test the extent of generalization induced by pretesting, while continuing training in another location (the 'training' location) until asymptotic learning was achieved. In addition, we tested how robust any generalization would be during continued training at the test location. In contrast to what could be expected from the findings by T. Zhang and colleagues (2010), a brief pre-test did not enable full transfer to the test location when orientation discrimination training in another location was continued for 15 sessions (Chapter 3). Moreover, we observed that the effect was only present in the very early phase of training in the test location, and then faded. The results thus illustrate that transfer due to a pre-test is restricted to early learning and has no bearing on the question whether asymptotic learning shows specificity or not. This puts claims by a number of recent studies (Wang et al., 2012; Xiao et al., 2008; J.-Y. Zhang et al., 2010) that skill can fully generalize in a 'double-training' paradigm in a proper perspective. We suggest that this interpretation is correct only for the early part of learning to which all of their studies were limited, and as such it is not in opposition with demonstrations of specificity of skill learning during asymptotic learning in earlier studies (Ahissar \& Hochstein, 1996; Crist et al., 1997; De Weerd, Vandenbussche, \& Orban, 1990; Karni \& Sagi, 1991; Schoups et al., 1995).

We also tested whether extensive stimulation outside awareness would lead to an advantage for subsequent learning, as this is a test of low-level plasticity induced by stimulation without attention and thus insulated from read-out effects. In previous studies, exposure outside awareness has been shown to induce advantages for learning (Gutnisky, Hansen, Iliescu, \& Dragoi, 2009; Watanabe, Náñez, \& Sasaki, 2001). However, in all of these studies, the advantage was either demonstrated only for the duration of a single session or exposure was intermixed with training. To demonstrate the robustness of any advantage of exposure outside awareness, it is crucial to show that the effect gives an advantage for the subsequent course of learning in the exposed location and for the exposed stimuli. However, similarly to 
the pre-test, no long-lasting beneficial effects were found. To our knowledge, other studies investigating effects of exposure on discrimination performance never followed up exposure by prolonged regular training to evaluate the longevity of the effects (see Gutnisky et al., 2009; Watanabe et al., 2001; T. Zhang et al., 2010). Our data show that any beneficial effects of exposure are limited to early phases of learning.

The data from Chapters 2 and 3 when combined suggest a complicated cooperation of read-out and low-level plasticity that does not necessarily agree with the original conceptions of neither the lowest-level theory nor the reverse hierarchy theory of perceptual learning. At the same time, proponents of double training paradigms seem to misinterpret the predictions of lowest-level and reverse hierarchy theories, thereby creating ill-posed theoretical oppositions.

In both reverse hierarchy and lowest-level theory, early stages of learning are thought to be not specific for stimulus features and stimulus location. In the design of the original double-training paradigm studies (Wang et al., 2012; Xiao et al., 2008; J.-Y. Zhang et al., 2010), generalization was tested only from a trained to a pre-exposed location (e.g. with training on an irrelevant feature), but not to other locations. In these studies, it is not certain to what extent there would have been also generalization to completely unstimulated locations. Assuming these effects would be location specific, the underlying process would have to involve the formation of connections between high-level and lower-level areas in which the expertise from the trained location would be selectively applied to the test location (where irrelevant training or pre-exposure had taken place). To the best of our knowledge, the question of location specificity of generalization after double-training has not been addressed yet. Nevertheless, in a recent study, some data are reported that have some bearing on the question of generalization to completely unstimulated regions of the visual field. Wang and colleagues (Wang, Zhang, Klein, Levi, \& Yu, 2014) demonstrated an interesting phenomenon that they called 'the piggy-backing effect'. This effect consists of two findings. First, orientation and motion direction discrimination tasks showed much more generalization than Vernier and contrast learning tasks in typical double-training paradigms. Second, if one of the two more locally specific tasks (e.g. Vernier) was trained together (in the same or a different 
location, and concurrently or consecutively) with a task yielding more generalization (e.g., motion direction discrimination), then the normally location-specific task generalized to totally unstimulated quadrants. This suggests that in some conditions, high-level procedural knowledge related to the execution of a particular task can spread across the whole visual field. Although Wang et al. (2014) state that their data cannot be fully understood with current models, they seem to see these findings as support for the idea that visual perceptual learning is primarily a rule-driven process (Zhang et al., 2010), and as opposed to theories claiming stimulus specificity based on (interactions with) lower-level visual areas. However, in all of the studies of this group (Wang et al., 2012; Xiao et al., 2008; J.-Y. Zhang et al., 2010; T. Zhang et al., 2010), training is limited to seven sessions, without any evidence that learning asymptote was reached. Their findings thus should be seen as pertaining to the early phase of learning, where all existing models have emphasized for decades that the learning is rule-driven and that there should be little specificity. Hence, although these studies show interesting modulations of the extent of generalization during early learning, some of which deserve further research, the essence of their data is much more in agreement with existing models then they acknowledge. These investigators motivate their studies by the aim to come up with strategies to generalize visual skills with less effort in cases where visual retraining is necessary in cases of visual rehabilitation (e.g. amblyopia). Unfortunately, their (in our view) misinterpretation of their own data may only have the effect of raising false hope in the targeted clinical populations.

The different forms of generalization observed in several studies of skill learning from a training location to another location briefly exposed to irrelevant training is likely due to enhanced read-out, which may generalize across locations (and stimulus features) according to poorly understood principles. By contrast, the effects of 15 sessions of exposure outside consciousness (passive exposure) most likely yields a form of plasticity at early levels of the visual system that is achieved without readout. Interestingly, we found that passive exposure in the test location yielded a fairly immediate benefit during training in the test location, which disappeared after a few sessions. This suggests that read-out has access to the lowest levels of the visual system from the beginning of learning, which is not in line with 
reverse hierarchy theory (Ahissar \& Hochstein, 2004), which suggests that read-out from low levels is achieved late in learning. The direct access by readout to low levels of the visual system from the beginning of learning is in line however with the outcome of a recent TMS study from our group (De Weerd et al., 2012), in which offline TMS directed to V1 interfered with orientation discrimination learning from the first two sessions. The data also suggest that there are forms of plasticity at low levels that can be induced without attention and that can contribute to early learning, which is in line neither with the basic concepts of the lowest-level theory of perceptual learning (Karni \& Bertini, 1997) nor with reverse hierarchy (Ahissar \& Hochstein, 2004). Interestingly, whatever the effect is of passive exposure, the lack of an effect lasting into the phase of asymptotic learning indicates that full perceptual learning requires a daily interaction in each training session between bottom-up stimulation and attention/read-out in order to achieve stimulus-specific skill. To test whether the effects of passive exposure could be increased so that they would lead to an enhancement of asymptotic learning, one could consider manipulations that make feedback signals (that would be part of the task during which the passive stimulation is delivered) more potent, such as the use of fluid or food rewards after mild periods of restriction (Seitz, Kim, \& Watanabe, 2009), or perhaps monetary rewards.

\section{What is the nature of the process leading to stabilization of visual skill memory?}

Our psychophysical data showed that 15 sessions of training (spread over 4-5 weeks) were not enough to sufficiently consolidate memory traces to avoid subsequent interference by another task (Chapter 4). Thus, a classical consolidation interpretation of the data seems improbable. In addition, modelling the data showed best agreement with empirical data when the proportion of neurons consolidating was set to $0 \%$, providing further support for re-recruitment of overlapping populations in different tasks as an explanation for behavioral interference. Hence, behavioral interference is determined by population overlap, and not by the status of a time-limited consolidation process and accordingly not by the time interval between tasks. Interestingly, we found that full-fledged interference only occurs 
when the two tasks are actively performed (in our studies the two tasks involved training at the $135^{\circ}$ reference and the other task involved training at the $105^{\circ}$ and $165^{\circ}$ reference orientations). The data of Been et al. (2011) combined with those presented in Chapter 4 indicate that the order of the two tasks is not relevant when the tasks involve attention.

The notion that procedural memory traces might never fully segregate and become stable (Debiec, LeDoux, \& Nader, 2002; Lee, 2008; Nadel, Hupbach, Gomez, \& Newman-Smith, 2012; Nader \& Einarsson, 2010) strongly implies that flexibility in memory traces is more important than avoidance of interference. From the perspective of normal skill learning, this can be understood from an efficiency consideration. When learning a skill, it is more effective to retrieve and modify an existing memory trace than creating a separate memory trace for each level of expertise of the same skill. In ecologically valid situations, it may be rather unlikely that two skills are trained which recruit almost the same neural populations while at the same time requiring opposite change in connectivity. Hence, unlikely interference may be a reasonable price to pay for more efficient management of memory traces during normal skill learning.

Furthermore, we found that passive interference (by stimuli around the $105^{\circ}$ and $165^{\circ}$ references presented outside awareness) did not have an effect on existing memory traces for orientation discrimination at the $135^{\circ}$ reference orientation. This indicates that the connectivity state induced by an attentively executed task cannot be easily influenced by competing but more subtle adaptation like effects induced by passive exposure. It is only when competing stimuli (at the $105^{\circ}$ and $165^{\circ}$ references) were shown first outside awareness (passive exposure) that there was interference with subsequent acquisition of orientation discrimination at the $135^{\circ}$ reference orientation. This effect was highly time-dependent, suggesting a quickly decaying state of adaptation induced by passive exposure. In general, the passive interference effect reported in Chapter 5 combined with the relatively weak effects of passive exposure in promoting learning (limited to early learning) reported in Chapter 3 are in line with the idea that effective perceptual learning may involve a form of exposure-related adaptation, which requires however a combination with 
attention for it to be transitioned into a more robust memory trace. The memory trace induced by an actively performed task is robust in the sense that it will receive only minor benefits from compatible passive stimuli and no interference from competitive passive stimuli. Note that robustness is merely meant to indicate a stable dormant memory trace, which however can be re-activated and modified by relevant experiences.

The data from active and passive interference experiments are in favor of the concept that memory traces can me modified by new experiences. This is line with neurophysiological and molecular biological evidence showing mechanisms for the dynamic formation and pruning of spines and synapses depending on calciumdependent and other mechanisms (Segal, Korkotian, \& Murphy, 2000). In addition, specific mechanisms have been described for the dynamic growth and shrinkage of existing spines (Calabrese, Saffin, \& Halpain, 2014; Mataga, Mizuguchi, \& Hensch, 2004). In mice it has been shown that a brief exposure to monocular deprivation early in life, which still permits normal adult distributions of binocularity, in these animals permits fast effects of a new phase of adult monocular deprivation, whereas in normally reared mice adult monocular deprivation would have much less effect (Hofer, Mrsic-Flogel, Bonhoeffer, \& Hübener, 2006). This can be explained by assuming that an early experience will leave a memory trace (embedded in shrunk spines and weaker synapses) that can be reactivated by specific patterns of stimulation (thanks to quick growth of existing spines and synaptic strengthening). This approach has been used to investigate and understand complex aspects of memory formation (Vetere et al., 2013) and provides a theoretical framework for understanding benefits and limits of therapeutical approaches for pathological conditions based on memory manipulation (Kindt, Soeter, \& Vervliet, 2009).

\section{What is the link between attention, microsaccades and the attentional blink effect?}

The work in the previous chapters indicates that attention is crucial for normal skill learning. At the end of the thesis, we included work specifically directed at investigating attentional limitations as revealed in the attentional blink paradigm. In this paradigm, classic studies (Raymond, Shapiro, \& Arnell, 1992, 1995; Shapiro, 
1994; Shapiro, Raymond, \& Arnell, 1997; Ward, Duncan, \& Shapiro, 1996) have demonstrated that after accurate discrimination of a first target presented in a rapid serial visual presentation (RSVP) stream a second target following too soon after the first suffers from reduced detectability or discriminability. This period of reduced visual performance is called the attentional blink. Our results showed a robust inhibition of microsaccade rate $200-300 \mathrm{~ms}$ after presentation of the first target, the time window commonly associated with the attentional blink effect (Chapter 6). Subsequently, a rebound of microsaccade rate was observed which coincided with increases in detection performance of the second target. Both the extent of microsaccade inhibition and microsaccade rebound were significantly correlated with participants' performance of detecting the second target letter at the time interval where the attentional blink was greatest. One possible interpretation of the results is that temporary withdrawal of attentional resources goes along with an inhibition of microsaccade rate.

The interpretation that microsaccades are an index for attentional allocation has received quite some support (Engbert \& Kliegl, 2003; Hafed \& Clark, 2002; Laubrock, Engbert, Rolfs, \& Kliegl, 2007; Yuval-Greenberg, Merriam, \& Heeger, 2014) but also opposition (Horowitz, Fine, Fencsik, Yurgenson, \& Wolfe, 2007; Tse, 2004; Tse, Sheinberg, \& Logothetis, 2002). One important open question is whether microsaccades are the cause or the effect of the observed attentional deficit. In addition, there is a possible alternative interpretation that the attentional blink is due to microsaccade suppression but not attentional processes. This view is rather provoking but should not be discarded right away. For example, if the slow rhythm of microsaccades is reset by $\mathrm{T} 1$, and if the presentation of $\mathrm{T} 2$ coincides with a period of lower probability of microsaccades, this may be related to the attentional blink. A possible underlying mechanism may be that microsaccades around presentation of a stimulus may enhance its processing, and the absence of microsaccades may lead to less processing (or even fading) (Martinez-Conde, Macknik, \& Hubel, 2004; Schroeder, Wilson, Radman, Scharfman, \& Lakatos, 2010).

However, if the link between attention and microsaccades can be supported in further experiments, it will be interesting to test the relevance of microsaccades for skill learning. Microsaccades may fulfill the function of actively sensing. In the 
active sensing hypothesis, receptor organs are given an active role in the exploration and collection of information, rather than a passive or purely receptive role (Schroeder et al., 2010). Microsaccades in primates may increase in frequency when visual information needs to be processed, similar to how rodents increase the frequency of whisking movements when they need to collect somatosensory information. In the field of visual skill learning, it will be interesting to test whether the frequency of (micro)saccades decreases as visual expertise in a task increases. This could be expected if information processing per (micro)saccade becomes more efficient. Such result would be in line with the idea that the performance of a visual skill would become more automatic (and requires less attention) as asymptotic learning progresses. Testing the relationships between attention, (micro)saccades and visual skill learning will be an interesting topic for future research.

\section{In summary}

Our data confirmed the importance of asymptotic learning to induce specificity. This provides the proper context to interpret a number of recent studies suggesting that skill can be generalized easily across stimulus dimensions and locations. Our data also show that while there is an exposure aspect and an associated adaptation state that can contribute to perceptual learning and interference, these effects are small compared to the contribution of attention. Interestingly, we found that effects of passive exposure could be taken advantage of by latter attentive learning, but that the benefit was limited to early learning. Since the passive exposure most likely induced effects in low-level areas, this shows that read-out mechanisms have access to these early levels from the beginning of learning. On the other hand, the effects of passive learning do not transfer to asymptotic learning, again emphasizing the importance of combining exposure with attention for normal skill learning. These findings emphasize the importance of interactions between read-out and bottom-up stimulation, but in a manner not predicted by current theories of perceptual leaning. Moreover, we present evidence that memory traces can be reactivated and modified, even after many weeks of training, adding evidence against consolidation models of learning. Finally, we have suggestive evidence for the contribution of microsaccades in the allocation of attention, which, if confirmed, opens important questions about 
the contribution of modifications in temporal patterns of microsaccades to improvements in skill performance as a function of daily training. 


\section{References}

Aberg, K. C., Tartaglia, E. M., \& Herzog, M. H. (2009). Perceptual learning with chevrons requires a minimal number of trials, transfers to untrained directions, but does not require sleep. Vision Research, 49(16), 2087-2094.

Ahissar, M., \& Hochstein, S. (1996). Learning pop-out detection: Specificities to stimulus characteristics. Vision Research, 36(21), 3487-3500.

Ahissar, M., \& Hochstein, S. (2004). The reverse hierarchy theory of visual perceptual learning. Trends in Cognitive Sciences, 8(10), 457-464.

Calabrese, B., Saffin, J.-M., \& Halpain, S. (2014). Activity-dependent dendritic spine shrinkage and growth involve downregulation of cofilin via distinct mechanisms. PLoS One, 9(4).

Crist, R. E., Kapadia, M. K., Westheimer, G., \& Gilbert, C. D. (1997). Perceptual learning of spatial localization: Specificity for orientation, position, and context. Journal of Neurophysiology, 78(6), 2889-2894.

De Weerd, P., Reithler, J., van de Ven, V., Been, M., Jacobs, C., \& Sack, A. T. (2012). Posttraining transcranial magnetic stimulation of striate cortex disrupts consolidation early in visual skill learning. The Journal of Neuroscience, 32(6), 1981-1988.

De Weerd, P., Vandenbussche, E., \& Orban, G. (1990). Speeding up visual discrimination learning in cats by differential exposure of positive and negative stimuli. Behavioural brain research, 36(1), 1-12.

Debiec, J., LeDoux, J. E., \& Nader, K. (2002). Cellular and systems reconsolidation in the hippocampus. Neuron, 36(3), 527-538.

Engbert, R., \& Kliegl, R. (2003). Microsaccades uncover the orientation of covert attention. Vision Research, 43(9), 1035-1045.

Fahle, M., Edelman, S., \& Poggio, T. (1995). Fast perceptual learning in hyperacuity. Vision Research, 35(21), 3003-3013.

Gutnisky, D. A., Hansen, B. J., Iliescu, B. F., \& Dragoi, V. (2009). Attention alters visual plasticity during exposure-based learning. Current Biology, 19(7), 555-560.

Hafed, Z. M., \& Clark, J. J. (2002). Microsaccades as an overt measure of covert attention shifts. Vision Research, 42(22), 2533-2545.

Hofer, S. B., Mrsic-Flogel, T. D., Bonhoeffer, T., \& Hübener, M. (2006). Prior experience enhances plasticity in adult visual cortex. Nature neuroscience, 9(1), $127-132$. 
Horowitz, T. S., Fine, E. M., Fencsik, D. E., Yurgenson, S., \& Wolfe, J. M. (2007). Fixational eye movements are not an index of covert attention. Psychological Science, 18(4), 356-363.

Hung, S.-C., \& Seitz, A. R. (2014). Prolonged training at threshold promotes robust retinotopic specificity in perceptual learning. The Journal of Neuroscience, 34(25), 8423-8431.

Jeter, P. E., Dosher, B. A., Liu, S.-H., \& Lu, Z.-L. (2010). Specificity of perceptual learning increases with increased training. Vision Research, 50(19), 1928-1940.

Karni, A., \& Bertini, G. (1997). Learning perceptual skills: Behavioral probes into adult cortical plasticity. Current Opinion in Neurobiology, 7(4), 530-535.

Karni, A., \& Sagi, D. (1991). Where practice makes perfect in texture discrimination: Evidence for primary visual cortex plasticity. Proceedings of the National Academy of Sciences, 88(11), 4966-4970.

Kindt, M., Soeter, M., \& Vervliet, B. (2009). Beyond extinction: Erasing human fear responses and preventing the return of fear. Nature neuroscience, 12(3), 256-258.

Laubrock, J., Engbert, R., Rolfs, M., \& Kliegl, R. (2007). Microsaccades are an index of covert attention: Commentary on Horowitz, Fine, Fencsik, Yurgenson, and Wolfe (2007). Psychological Science, 18(4), 364-366; discussion 367-368.

Lee, J. L. (2008). Memory reconsolidation mediates the strengthening of memories by additional learning. Nature neuroscience, 11(11), 1264.

Martinez-Conde, S., Macknik, S. L., \& Hubel, D. H. (2004). The role of fixational eye movements in visual perception. Nature Reviews Neuroscience, 5(3), 229-240.

Mataga, N., Mizuguchi, Y., \& Hensch, T. K. (2004). Experience-dependent pruning of dendritic spines in visual cortex by tissue plasminogen activator. Neuron, 44(6), 1031-1041.

Nadel, L., Hupbach, A., Gomez, R., \& Newman-Smith, K. (2012). Memory formation, consolidation and transformation. Neuroscience \& Biobehavioral Reviews, 36(7), 1640-1645.

Nader, K., \& Einarsson, E. Ö. (2010). Memory reconsolidation: An update. Annals of the New York Academy of Sciences, 1191(1), 27-41.

Raymond, J. E., Shapiro, K. L., \& Arnell, K. M. (1992). Temporary suppression of visual processing in an rsvp task: An attentional blink? Journal of Experimental Psychology: Human Perception and Performance, 18(3), 849.

Raymond, J. E., Shapiro, K. L., \& Arnell, K. M. (1995). Similarity determines the attentional blink. Journal of Experimental Psychology: Human Perception and Performance, 21(3), 653. 
Schoups, A. A., Vogels, R., \& Orban, G. A. (1995). Human perceptual learning in identifying the oblique orientation: Retinotopy, orientation specificity and monocularity. Journal of Physiology, 483(Pt 3), 797-810.

Schroeder, C. E., Wilson, D. A., Radman, T., Scharfman, H., \& Lakatos, P. (2010). Dynamics of active sensing and perceptual selection. Current Opinion in Neurobiology, 20(2), 172-176.

Segal, M., Korkotian, E., \& Murphy, D. D. (2000). Dendritic spine formation and pruning: Common cellular mechanisms? Trends in Neurosciences, 23(2), 53-57.

Seitz, A. R., Kim, D., \& Watanabe, T. (2009). Rewards evoke learning of unconsciously processed visual stimuli in adult humans. Neuron, 61(5), 700-707.

Shapiro, K. L. (1994). The attentional blink: The brain's" eyeblink". Current Directions in Psychological Science, 86-89.

Shapiro, K. L., Raymond, J. E., \& Arnell, K. M. (1997). The attentional blink. Trends in Cognitive Sciences, 1(8), 291-296.

Tse, P. U. (2004). Mapping visual attention with change blindness: New directions for a new method. Cognitive Science, 28(2), 241-258.

Tse, P. U., Sheinberg, D. L., \& Logothetis, N. K. (2002). Fixational eye movements are not affected by abrupt onsets that capture attention. Vision Research, 42(13), 1663-1669.

Vetere, G., Piserchia, V., Borreca, A., Novembre, G., Aceti, M., \& AmmassariTeule, M. (2013). Reactivating fear memory under propranolol resets pre-trauma levels of dendritic spines in basolateral amygdala but not dorsal hippocampus neurons. Frontiers in behavioral neuroscience, 7.

Wang, R., Zhang, J.-Y., Klein, S. A., Levi, D. M., \& Yu, C. (2012). Task relevancy and demand modulate double-training enabled transfer of perceptual learning. Vision Research, 61(0), 33-38.

Wang, R., Zhang, J.-Y., Klein, S. A., Levi, D. M., \& Yu, C. (2014). Vernier perceptual learning transfers to completely untrained retinal locations after double training: A "piggybacking" effect. Journal of Vision, 14(13), 12-12.

Ward, R., Duncan, J., \& Shapiro, K. L. (1996). The slow time-course of visual attention. Cognitive Psychology, 30(1), 79-109.

Watanabe, T., Náñez, J. E., \& Sasaki, Y. (2001). Perceptual learning without perception. Nature, 413(6858), 844-848.

Xiao, L.-Q., Zhang, J.-Y., Wang, R., Klein, S. A., Levi, D. M., \& Yu, C. (2008). Complete transfer of perceptual learning across retinal locations enabled by double training. Current Biology, 18(24), 1922-1926. 
Yuval-Greenberg, S., Merriam, E. P., \& Heeger, D. J. (2014). Spontaneous microsaccades reflect shifts in covert attention. The Journal of Neuroscience, 34(41), 13693-13700.

Zhang, J.-Y., Zhang, G.-L., Xiao, L.-Q., Klein, S. A., Levi, D. M., \& Yu, C. (2010). Rule-based learning explains visual perceptual learning and its specificity and transfer. The Journal of Neuroscience, 30(37), 12323-12328.

Zhang, T., Xiao, L.-Q., Klein, S. A., Levi, D. M., \& Yu, C. (2010). Decoupling location specificity from perceptual learning of orientation discrimination. Vision Research, 50(4), 368-374. 


\section{KNOWLEDGE VALORIZATION}




\section{Relevance}

Learning and memory are a fundamental part of human life. Lesion-induced memory impairments, such as those of Henry Molaison, more commonly known in the scientific world as H.M., impressively illustrate how big a part they actually play. Importantly though, lesions have also contributed to the insight that there is not just a single memory type. Instead, a distinction is made between explicit and implicit memory. Each type is again subdivided into several subtypes, and for implicit memory one of these is procedural memory, which involves the acquisition of skills. Skills can be related to different domains or combinations of them, namely cognitive, motor, and perceptual. Perceptual learning, in turn can relate to any of the five senses, visual olfactory, somatosensory, and gustatory. In the present thesis we focused on visual perceptual learning and attention. Given the above classification of memory, it becomes obvious that visual perceptual learning is just one small fraction of the memory concept. This is not to say that perceptual learning is unimportant though. As almost any leisurely, educational or professional activity one performs relies on one or more skills (see General Introduction), it is important to acquire more knowledge about visual perceptual learning but also skill learning in general. A considerable part of education at school (languages, mathematics, sciences) relies heavily on skill learning which in itself makes skill learning a very important and relevant domain of cognitive neuroscience.

\section{To whom are the research results of interest?}

The empirical work presented here first and foremost was aimed to acquire fundamental knowledge about visual perceptual learning, the nature of underlying neural networks and processes, as well as the relationship between attention and microsaccades. Therefore, the findings presented here are mostly of interest for researchers in the field of skill learning, memory, and attention. The results might, for example, inspire further empirical work regarding computational modeling of the visual system.

Besides, our findings might also be of interest for policy makers in education. Findings about specificity/generalization (Chapters 2 and 3) and interference (Chapters 4 and 5) in the domain of visual perceptual learning might have general 
relevance for the organization of teaching in schools and universities, and for professional training in professions requiring specific skills. We have shown that interference occurs even for strictly sequential training (Chapter 4) and with unattended/unperceived stimulation (Chapter 5). In essence, our data show that a reactivated memory trace under some conditions can be erased by additional learning (interference), whereas in other conditions additional training strengthens or refines the memory trace. In the laboratory situations we used, and using simple tasks, we managed to control when strengthening vs. erasure occurred. It is therefore an interesting question in real-life learning tasks in schools or professional environments which conditions need to be created to strengthen learning, and which conditions should be avoided to avoid interference. That question is however very challenging as the number of relevant factors in complex environments and complex learning tasks increases by orders of magnitude. It will be necessary to gradually build up the complexity of skill learning tasks to approach real-life tasks to achieve a better understanding of learning conditions that could either enhance or interfere with real-life learning.

Our findings may also have clinical relevance. It has been suggested that some visual deficits can be counteracted by training. In the visual domain, it has been suggested that visual perceptual learning might be used in the treatment of amblyopia (e.g., Chen, Chen, Fu, Chien, \& Lu, 2008; Fronius, Cirina, Cordey, \& Ohrloff, 2005; Levi \& Polat, 1996; Li, Provost, \& Levi, 2007; Zhou et al., 2006), a developmental abnormality that is caused by physiological alterations in the visual cortex (Kiorpes \& McKeet, 1999). This condition can be ameliorated by training in human children and adults (Kandel, Schwartz, Jessell, Siegelbaum, \& Hudspeth, 2012). As research has shown that visual perceptual learning results in performance improvements that have been reported to remain stable for years (Karni \& Sagi, 1993), it is at least worth to investigate further in how far perceptual learning can be used to aid/speed up conventional amblyopia treatment such as patching the good eye (for a critical review see, Levi \& Li, 2009). The idea of training has been demonstrated to be useful as well in recovering from stroke (e.g., to overcome partial paralysis or impairment of language) (Meinzer, Elbert, Djundja, Taub, \& Rockstroh, 2006; Taub, Uswatte, \& Pidikiti, 1999), and in preventing maladaptive 
topographic remapping after amputations (Chan et al., 2007; Ramachandran \& Altschuler, 2009). Although far removed from the experiments performed in the thesis, the theoretical idea of reactivating memory traces and then deleting them by imposing interference (either behaviorally or pharmacologically) has been used successfully in combatting phobia's and psychological trauma Kindt, Soeter, \& Vervliet, 2009; Soeter \& Kindt, 2011). One might speculate whether such approaches might also be useful in combatting maladaptive or pathological habits (such as neurotic behaviors or addictions). Given the fact that basal ganglia/striatum have been implicated both in habit learning and addictions this idea is not too farfetched, but as indicated in the previous section, much fundamental research is required before applications will come in sight.

\section{What can result from the research findings?}

As emphasized above, the studies in this thesis were conducted to obtain more knowledge about perceptual learning and attention, and did not anticipate any concrete products, services, processes, activities or commercial activities. The field of perceptual learning is a rather small niche in the field of cognitive neuroscience. Our research aims at learning about perceptual learning - simply because we want to understand how it works. If, as is the case with amblyopia, treatment opportunities arise from such research that is marvelous. But one should not be ignorant about the fact that most of the time fundamental science comes before applied science, and that the ways in which fundamental science leads to applications are almost always complex, dependent on convergent insights from uncountable studies, and simply unpredictable. This is why the across-the-board requirement to write a valorization chapter by Maastricht University is hard to understand - it suggests that the leadership of our university understands neither the value of fundamental science nor its complex relationship with societal and economical applications. Fundamental science constitutes one of the noblest human endeavors - the curiosity to gain deep understanding into all that surrounds us including other humans - and as such is highly intrinsically valuable. Whether or not there is a potential for material or monetary gain or any type of societal impact should be irrelevant. The better universities defend this academic view of science against various political pressures, 
the richer science will be and the better chances will be for beneficial applications. Writing a 'valorization chapter' and treating fundamental science as something that it is not, as Maastricht University now requires from its $\mathrm{PhD}$ students, certainly is not creating applied value. It is just a waste of valuable time.

\section{References}

Chan, B. L., Witt, R., Charrow, A. P., Magee, A., Howard, R., Pasquina, P. F., . . . Tsao, J. W. (2007). Mirror therapy for phantom limb pain. New England Journal of Medicine, 357(21), 2206-2207.

Chen, P. L., Chen, J. T., Fu, J. J., Chien, K. H., \& Lu, D. W. (2008). A pilot study of anisometropic amblyopia improved in adults and children by perceptual learning: An alternative treatment to patching. Ophthalmic and Physiological Optics, 28(5), $422-428$.

Fronius, M., Cirina, L., Cordey, A., \& Ohrloff, C. (2005). Visual improvement during psychophysical training in an adult amblyopic eye following visual loss in the contralateral eye. Graefe's Archive for Clinical and Experimental Ophthalmology, 243(3), 278-280.

Kandel, E. R., Schwartz, J. H., Jessell, T. M., Siegelbaum, S. A., \& Hudspeth, A. (2012). Principles of neural science (5 ed.): McGraw-Hill New York.

Karni, A., \& Sagi, D. (1993). The time course of learning a visual skill. Nature, 365(6443), 250-252.

Kindt, M., Soeter, M., \& Vervliet, B. (2009). Beyond extinction: Erasing human fear responses and preventing the return of fear. Nature Neuroscience, 12(3), 256-258.

Kiorpes, L., \& McKeet, S. P. (1999). Neural mechanisms underlying amblyopia. Current Opinion in Neurobiology, 9(4), 480-486.

Levi, D. M., \& Li, R. W. (2009). Perceptual learning as a potential treatment for amblyopia: A mini-review. Vision Research, 49(21), 2535-2549.

Levi, D. M., \& Polat, U. (1996). Neural plasticity in adults with amblyopia. Proceedings of the National Academy of Sciences, 93(13), 6830-6834.

Li, R. W., Provost, A., \& Levi, D. M. (2007). Extended perceptual learning results in substantial recovery of positional acuity and visual acuity in juvenile amblyopia. Investigative Ophthalmology \& Visual Science, 48(11), 5046-5051.

Meinzer, M., Elbert, T., Djundja, D., Taub, E., \& Rockstroh, B. (2006). Extending the constraint-induced movement therapy (cimt) approach to cognitive functions: 
Constraint-induced aphasia therapy (ciat) of chronic aphasia. NeuroRehabilitation, 22(4), 311-318.

Ramachandran VS \& Altschuler EL. (2009). The use of visual feedback, in particular mirror visual feedback, in restoring brain function. Brain, 1693-1710.

Soeter, M., \& Kindt, M. (2011). Disrupting reconsolidation: Pharmacological and behavioral manipulations. Learning \& Memory, 18(6), 357-366.

Taub, E., Uswatte, G., \& Pidikiti, R. (1999). Constraint-induced movement therapy: A new family of techniques with broad application to physical rehabilitation-a clinical review. Journal of rehabilitation research and development, 36(3), 237-251.

Zhou, Y., Huang, C., Xu, P., Tao, L., Qiu, Z., Li, X., \& Lu, Z.-L. (2006). Perceptual learning improves contrast sensitivity and visual acuity in adults with anisometropic amblyopia. Vision Research, 46(5), 739-750. 


\section{Acknowledgements}

Art is I; science is we.

\section{Claude Bernard}

The 'we' in science for me also refers to all the people who made it happen!

Firstly, I would like to thank Peter who has been my supervisor since my student assistant days during the research master. Thanks for all your support, advice and trust throughout the years - it means a lot to me. We share a love for solidly designed psychophysical experiments and even though having yet another control experiment can drive one crazy at moments it just shows that you love to do science meticulously and don't take shortcuts. I truly admire your writing skills and am thrilled that we never reached a $20^{\text {th }}$ version of an article. -$)$

Mark, the attentional blink project would not have happened without you and I am thankful that you never gave up on it even though getting the paradigm to work was a true pain. Thanks for pushing things to meet my thesis deadlines and all the proofreading you have done. I would also like to thank the other (former) members of our research group who all contributed in one way or another: Marin, Bert, Alexandra, and Eric. After months of only writing and teaching it is great to be involved again in new and exciting research projects of our PALM group!

I think room mates are necessary to keep you sane when doing a $\mathrm{PhD}$. So, my thanks go to Eva, Charlotte, Martin and Emma! It was great to have someone to share happy, sad and frustrating moments with. I could always count on you to join in for a good chat, coffee or lunch.

Anna, we share a love for food and baking and I would babysit your sourdough anytime again! We have travelled together through Italy, Germany and the Netherlands and you were there when I learned to conquer my fear of rollercoasters 
(When do we go again?). It is great how you always find time to do stuff together even though you have such a busy schedule and we don't plan ahead that much. My teaching schedule makes me an unreliable factor for common lunches, but nevertheless you never stopped asking me whether I would like joining. I am going to miss you a lot once you will move back to Italy and we will make sure to visit you there!

Emma, we also share a passion for food and seem to have strangely synced emotional episodes. I very much enjoyed our chats across office desks and the coffees we have shared. I admire you for all the strength you have brought up the past couple of months to cope with everything that was thrown at you. I am looking forward to you spending more time in Maastricht soon and I am confident that things with the scanner will work out in the end - it is just a matter of keeping sane in the mean time!

Food is important, you might have realized by now, and also with Anke, Sven, Lars, Roberta, and Britta it was about us getting together and get cooking. I remember sushi (both, perfect and complete failure), tapas, homemade pasta (even the umbrella kind), curries, vegetarian dinners, salt-crusted fish, liquid-center chocolate fondant and so much more. But besides all the cooking you have become true friends and I hope we will continue our cooking adventures until we are grey, wrinkled and old. (:)

Thomas M., you also have been there since the first days of our bachelor and ever since. I value your calm and laid-back attitude and you are welcome to stay at our place anytime. Our time in a crappy student house in Heugem with a courtyard and self-build BBQ will always have a special place in my memory.

The Cognitive Neuroscience department is a truly great place to work at and that is mainly because of all the amazing colleagues! Thank you Judith E., Tahnée, Helen, Lukas, Valentin, Mario A., Franzi, Thomas E., Matteo, Mario S., Nina, Christianne, Teresa, Jeannette, Gojko, Joao, Tom, Michelle, Aline, Sanae, Inge, Martin F., 
Michael, Valerie, Rob. Also, I would like to thank Riny, Christel, Annemie, and Eva for their great help with all kind of things!

A big thank you goes to the choir, more specifically the Altos. Singing is the best remedy to get everything work-related out of your head. Just seeing how Wim is putting in all this energy to make us the best University Choir of Maastricht Monday after Monday makes you reconsider things. Special thanks go to Nicola, Beate, and Klaartje.

Irma, Rudi, Hubert, und Ursl, danke dass ihr an mich geglaubt und mich unterstützt habt, dass zu tun was ich wirklich interessant finde. Malin, du bist eine der willensstärksten Personen die ich kenne und ich bin mir sicher, dass du alles erreichen kannst was du dir in den Kopf setzt. Die nächste Dissertation, die wir gemeinsam feiern werden, wird deine sein! Timon, du bist die Ruhe in Person und ich beneide dich für die Gelassenheit und Zufriedenheit die du ausstrahlst. Let's finish Chuck! Finja, mögen wir uns äußerlich auch nicht so ähneln, innerlich bist du mir ganz nahe. Du teilst meine Begeisterung für Bücher, Pferde, und das Kochen. Ich bin stolz wie du alles gemeistert hast und was für ein toller Mensch du geworden bist!

Felix you are truly awesome! You have been there right from the start of my time in Maastricht almost twelve years ago. Ever since you have been there for me and supported me. Sometimes that meant giving me a little push when I needed it, cooking some dinner so I would get some energy back, or just telling me that I would be able to finish this thesis and make it something I could be proud of. You always believe in me and that means the world to me! 


\section{Curriculum Vitae}

Gesa Lange was born in Oelde, Germany on June $15^{\text {th }} 1982$. She graduated with the secondary school certificate in 2002 at the Gymnasium Laurentianum in Warendorf. In 2004, she enrolled at the Faculty of Psychology and Neuroscience at Maastricht University. During the final year of her undergraduate studies she participated in an exchange program and spent a semester in Victoria, Canada. She obtained her Bachelor of Science in Biological Psychology in 2007 and subsequently followed the Research Master program in Cognitive Neuroscience at Maastricht University. During that time she worked as a student assistant in the research group of Professor Peter De Weerd. In 2008 she obtained the HSP Huygens Scholarship for International Students. In her Master thesis, under supervision of Professor Peter De Weerd, she conducted empirical work investigating biased competition in humans using fMRI. She obtained her Master of Science, cum laude, in Cognitive Neuroscience in 2009. She then started the PhD program, under the supervision of Professor Peter De Weerd and Mark J. Roberts, at the Department of Cognitive Neuroscience at the Faculty of Psychology and Neuroscience. She is currently a post-doctoral fellow at the same institute where she continues her research and teaches at bachelor and master level. 


\section{Articles}

Lange, G., Lowet, E., Roberts, M. J., \& De Weerd, P. (in preparation). Training in visual orientation discrimination yields limited position and orientation specificity after asymptotic learning.

Lange, G. \& De Weerd, P. (submitted). Limited transfer of orientation discrimination performance after pre-test and masked exposure.

Lange, G.*, Senden, M.*, Radermacher, A., Goebel, R., \& De Weerd, P. (in preparation). Long-term successive training causes behavioral interference.

Lange, G., Radermacher, A., Jans, B., \& De Weerd, P. (in preparation). Interference with visual skill learning in humans by exposure to masked, unattended stimuli.

Roberts, M. J.*, Lange, G.*, Lowet, E., \& De Weerd, P. (in preparation). The attentional blink effect is related to microsaccade suppression but not rebound.

* shared first authorship

\section{Conference publications}

Senden, M., Lange G., Radermacher, A., Goebel, R., De Weerd, P. (2015). Successive training of two tasks in three-week periods still leads to behavioral interference. Poster presented at the annual meeting of the Society for Neuroscience 2015, Chicago, USA.

Lange G., Radermacher, A., Been, M., Jans, B., De Weerd, P. (2012). Passive interference of perceptual visual learning in humans. Poster presented at the annual meeting of the Society for Neuroscience 2012, New Orleans, USA

Lange G., De Weerd, P. (2011). Attention overrules adaptation in human extrastriate cortex. Poster presented at the annual meeting of the Society for Neuroscience 2011, Washington DC, USA 